\title{
Cross-cultural intelligence amid intricate cultural webs : A tale of the UnDutchables in the land of 1001 smiles
}

Citation for published version (APA):

Kwanjai, N. N. (2011). Cross-cultural intelligence amid intricate cultural webs : A tale of the UnDutchables in the land of 1001 smiles. [Doctoral Thesis, Maastricht University]. Maastricht University. https://doi.org/10.26481/dis.20110217nk

Document status and date:

Published: 01/01/2011

DOI:

10.26481/dis.20110217nk

Document Version:

Publisher's PDF, also known as Version of record

\section{Please check the document version of this publication:}

- A submitted manuscript is the version of the article upon submission and before peer-review. There can be important differences between the submitted version and the official published version of record.

People interested in the research are advised to contact the author for the final version of the publication, or visit the DOI to the publisher's website.

- The final author version and the galley proof are versions of the publication after peer review.

- The final published version features the final layout of the paper including the volume, issue and page numbers.

Link to publication

\footnotetext{
General rights rights.

- You may freely distribute the URL identifying the publication in the public portal. please follow below link for the End User Agreement:

www.umlib.nl/taverne-license

Take down policy

If you believe that this document breaches copyright please contact us at:

repository@maastrichtuniversity.nl

providing details and we will investigate your claim.
}

Copyright and moral rights for the publications made accessible in the public portal are retained by the authors and/or other copyright owners and it is a condition of accessing publications that users recognise and abide by the legal requirements associated with these

- Users may download and print one copy of any publication from the public portal for the purpose of private study or research.

- You may not further distribute the material or use it for any profit-making activity or commercial gain

If the publication is distributed under the terms of Article $25 \mathrm{fa}$ of the Dutch Copyright Act, indicated by the "Taverne" license above, 


\title{
Cross-cultural Intelligence amid Intricate Cultural Webs
}

\author{
A Tale of the UnDutchables \\ in the Land of 1001 Smiles
}

Nantawan Noi Kwanjai

นันทวักธ์ แ้อง Vรัมิจ 
(C) 2011 Nantawan Noi Kwanjai, Maastricht, the Netherlands. All rights reserved.

Kwanjai, Nantawan Noi

Cross cultural intelligence amid intricate cultural webs A tale of the UnDutchables in the land of 1001 smiles $^{\S}$

$\S$ The term 'the UnDutchables' is respectfully acknowledged to have been coined by the authors of the book: The UnDutchables: an observation of the Netherlands, its culture and its inhabitants (White and Boucke 2006).

ISBN 9789461590060

Typeset: $\quad$ Nantawan Noi Kwanjai (with Microsoft ${ }^{\circledR}$ Office 2003, 2007).

Cover concept: Nantawan Noi Kwanjai.

Cover image: (C) 2008 Jason Rogers.

Publisher: Universitaire Pers Maastricht, the Netherlands.

Printer: Datawyse, Maastricht, the Netherlands. 


\title{
Cross-cultural Intelligence amid Intricate Cultural Webs
}

\section{A Tale of the UnDutchables in the Land of 1001 Smiles}

\author{
PROEFSCHRIFT \\ ter verkrijging van de graad van doctor \\ aan de Universiteit Maastricht, \\ op gezag van de Rector Magnificus Prof. mr. G.P.M.F. Mols \\ volgens het besluit van het College van Decanen, \\ in het openbaar te verdedigen \\ op donderdag 17 februari 2011 om 16.00 uur \\ door
}

\section{Nantawan Kwanjai}

เันทวักธ์ งวัพิจ

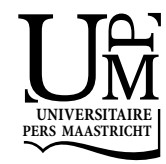




\section{Promotor}

Prof. dr. J. Friso den Hertog

\section{Beoordelingscommissie}

Prof. dr. Mariëlle Heiltjes (voorzitter)

Prof. dr. Maarten Verkerk

Prof. dr. Geert Duysters (Technische Universiteit Eindhoven) 
These are questions that do not have answers. This is what makes them interesting. Questions without answers compel us to search. And it is the search that is important, not the answer.

Krzysztof Kieslowski from an interview with Newsweek 1995

\section{.3334>8E.}

Do I dare

Disturb the universe?

In a minute there is time

For decisions and revisions which a minute will reverse.

T.S. Eliot The Love Song of J. Alfred Prufrock

\section{.333ब $\rightarrow 8 E$.}

Nothing in life is to be feared, it is only to be understood. Now is the time to understand more, so that we may fear less.

\section{$.3334 \rightarrow 88$.}

[...] and it is not necessary to know everything in order to understand something.

Clifford Geertz The Interpretation of Cultures

\section{. 3334मsE8.}

If knowledge is power, then understanding is peace. 
for the finest Thai and Dutch souls l've had a privilege to know ... my mother \& father
and Friso \& Arnold 


\section{TABLE OF CONTENTS}

Table of contents

Extended table of contents

List of diagrams

List of reflections

Acknowledgements

\section{Chapter 1}

\section{A road less travelled}

\section{'Sawasdee Kha'}

1.1. Theoretical domain and substantive platform 4

$\begin{array}{ll}\text { 1.2. Research strategy } & 10\end{array}$

$\begin{array}{ll}\text { 1.3. Research results } & 12\end{array}$

1.4. Style and organization of the manuscript 13

Chapter 2

Qualitative grounded theory case research

'To see the World in a Grain of Sand'

2.1. Interpretive grounded theory 20

2.2. Grounded theory: procedures and directives $\quad 27$

2.3. Qualitative case research 46

2.4. Evaluative concerns: the questions of rigor and relevance 53

\section{Chapter 3}

\section{Case ADT}

\section{UnDutching the UnDutchables}

3.1. Enter the UnDutchables

3.2. The accidental inheritance and its cultural riddles 78

3.3. Deciphering the cultural riddles 85

3.4. Final words 90 
Intricate cultural webs

\section{A metaphor we live by}

4.1. Images of culture: metaphors we live by 93

4.2. Culture $\quad 99$

$\begin{array}{lr}\text { 4.3. Intricate cultural webs } & 107\end{array}$

4.4. Core meanings: Dutch and Thai 113

Chapter 5

\section{Case CDW}

Portrait of an odd-eyed cat

5.1. Meet the odd-eyed alliance $\quad 126$

5.2. It takes two to tango $r$

5.3. Cultural crossing at CDW $r$

5.4. Final words $r$

Chapter 6

XCQ amid intricate cultural webs

A grounded theory

6.1. Cultural crossing

6.2. Cross-cultural intelligence (XCQ) 161

6.3. XCQ amid intricate cultural webs: a grounded theory $\quad 164$

6.4. Cross-cultural heuristic $r$

$\begin{array}{lr}\text { 6.5. XCQ within the CQ context } & 179\end{array}$

Chapter 7

Case ZIA

Unveiling a cultural chameleon

7.1. Dutch seed stumbling on Thai soil

7.2. A cultural chameleon in disguise?

7.3. Lessons from the cultural chameleon

7.4. Final words 
The road ahead

The journey is the destination.

8.1. Taking stock: contributions

8.2. The road ahead: future research

8.3. Back to the future: limitations

Bibliography

Nederlandse samenvatting

263

Summary in English

269

Author's biography

275 



\section{EXTENDED TABLE OF CONTENTS}

Table of contents

Extended table of contents

List of diagrams

List of reflections

Acknowledgements

\section{Chapter 1}

\section{A road less travelled}

\section{'Sawasdee Kha'}

1.1. Theoretical domain and substantive platform 4

The emergence of study title: a moment of reflexivity 5

Formal theoretical domain: cross-cultural intelligence (XCQ) 6

Substantive platform: Dutch-Thai cross-cultural interaction 8

1.2. Research strategy 10

$\begin{array}{ll}\text { Interpretive grounded theory } & 10\end{array}$

$\begin{array}{ll}\text { Qualitative case research } & 12\end{array}$

1.3. Research results 12

1.4. Style and organization of the manuscript 13

Chapter 2

\section{Qualitative grounded theory case research}

'To see the World in a Grain of Sand'

2.1. Interpretive grounded theory 20

Research paradigm $\quad 20$

Research paradigm landscape: a bird's-eye view $\quad 21$

This study in the paradigm landscape $\quad 23$

2.2. Grounded theory: procedures and directives 27

Elements, principles and output $\quad 31$

Research question $\quad 31$

Theoretical sampling 33

Evidence collection 40

Coding and theorizing $\quad 42$

Final deliverable $\quad 45$

Key directives $\quad 45$

2.3. Qualitative case research 46

Case, case study and case study research 46

What is a case? $\quad 46$

Case study v. case study research $\quad 47$

Qualitative case research: a multiple utility view of case study 49 
Case study as a structure of the inquiry

Case study as a grounded theorizing instrument $\quad 50$

Case study as a 'virtual reality' for reporting and learning 51

The virtual reality of thick descriptive cases $\quad 52$

Convention and poetic license $\quad 52$

Factual accuracy $\quad 53$

2.4. Evaluative concerns: the questions of rigor and relevance 53

Evaluative concerns for qualitative research 54

Identification and definition of qualitative evaluation $\quad 55$

Terminologies: What's in a name?

Evaluative Criteria for qualitative research: a qualitative 'holy trinity' 58

Methods and procedures to ensure rigor and relevance 64

\section{Chapter $3 \quad 69$}

\section{Case ADT}

\section{UnDutching the UnDutchables}

3.1. Enter the UnDutchables 72

Corporate 73

Local Division in Thailand $\quad 74$

The actors $\quad 75$

CEO: Dhr. Kees van Dijk (Khun Kees)

VP, Sales \& Marketing: Dhr. Joeri van Emmen (Khun Joeri)

MD, Thai Division: Dhr. Pim Kempster (Khun Pim) 76

Production manager/HRM: Khun Maleewan Manasakul (Khun Maleewan) 77

$\begin{array}{ll}\text { Supporting cast } & 78\end{array}$

3.2. The accidental inheritance and its cultural riddles 78

$\begin{array}{ll}\text { An accidental inheritance } & 79\end{array}$

Deadline: dead or alive? $\quad 80$

Three strikes and you're out $\quad 83$

The bottom line $\quad 84$

3.3. Deciphering the cultural riddles 85

A multitude of competing meanings

Time and commitment $\quad 85$

Motivation: pressure v. persuasion $\quad 86$

Mistake: shame or guilt? $\quad 88$

UnDutching as a prerequisite for cross-cultural encounter 88

Let's call the whole thing off $\quad 89$

Something's got to give $\quad 89$

3.4. Final words 90

Khun Kees $\quad 90$

$\begin{array}{lr}\text { Khun Joeri } & 90\end{array}$

Khun Pim $\quad 90$

$\begin{array}{lr}\text { Khun Maleewan } & 90\end{array}$ 
Intricate cultural webs

\section{A metaphor we live by}

4.1. Images of culture: metaphors we live by 93

Culture as the air or one's nose: the paradox of culture $\quad 94$

Culture as an iceberg or onion: visible and hidden cultural elements 95

Culture as software, mosaic, or webs: the complexity of cultural force 96

4.2. Culture

$\begin{array}{lr}\text { Culture: sense-making process and shared meanings } & 100\end{array}$

$\begin{array}{ll}\text { Cultural elements: a cultural onion model } & 104\end{array}$

$\begin{array}{ll}\text { Cultural core: core shared meanings } & 106\end{array}$

$\begin{array}{ll}\text { 4.3. Intricate cultural webs } & 107\end{array}$

$\begin{array}{ll}\text { Cultural unit } & 107\end{array}$

Notes on the concept of cultural unit $\quad 109$

$\begin{array}{ll}\text { Intricate cultural webs } & 110\end{array}$

Intricate cultural webs as cultural identity of a cultural unit 110

Intricate cultural webs as property of a cross-cultural interface and platform 112

Properties of intricate cultural webs $\quad 112$

4.4. Core meanings: Dutch and Thai 113

$\begin{array}{lr}\text { The meaning of 'self' } & 115 \\ & 117\end{array}$

$\begin{array}{ll}\text { The meaning of 'others' } & 117\end{array}$

$\begin{array}{ll}\text { The meaning of 'environment' } & 119\end{array}$

$\begin{array}{ll}\text { The meaning of '(inter)relationship' } & 120\end{array}$

\section{Chapter 5}

\section{Case CDW}

Portrait of an odd-eyed cat

5.1. Meet the odd-eyed alliance 126

$\begin{array}{ll}\text { The big picture } & 126\end{array}$

$\begin{array}{ll}\text { The origin } & 127\end{array}$

$\begin{array}{ll}\text { The evolution } & 127\end{array}$

Present day CDW 128

$\begin{array}{ll}\text { The Actors } & 129\end{array}$

Co-founder and first President: Dhr. Geert Halsema (Khun Geert) 129

Co-founder: Khun Chatoeran Prempreecha (Khun Chatoeran) 130

President: Dhr. Karel Westerveld (Khun Karel) 131

Executive secretary: Khun Busara Navara (Khun Busara) 132

Vice President: Khun Chaowalit Saenamuang (Khun Chaowalit) 133

Supporting cast $\quad 134$

5.2. It takes two to tango 134

$\begin{array}{ll}\text { An ideal match: a tale of two founders } & 135\end{array}$

$\begin{array}{ll}\text { A curious offspring: an odd-eyed cat? } & 137\end{array}$

$\begin{array}{ll}\text { Partnership of nationals } & 138\end{array}$

Partnership of professionals 140

Partnership of organizations 142 
Partnership of ranks $\quad 143$

De wereld draait door... $\quad 145$

5.3. Cultural crossing at CDW 145

Multi-level cultural partnership as strategic trademark of CDW 145

$\begin{array}{ll}\text { The significance of context } & 147\end{array}$

$\begin{array}{lr}\text { The value of cross-cultural instrument } & 148\end{array}$

5.4. Final words $r$

Khun Geert $\quad 149$

Khun Karel 149

Khun Busara $\quad 149$

Khun Chaowalit $\quad 149$

$X C Q$ amid intricate cultural webs

A grounded theory

$\begin{array}{lr}\text { 6.1. Cultural crossing } & 153\end{array}$

Modes of crossing in cross-cultural condition $\quad 154$

Clashing cross-cultural condition 156

Reciprocal cross-cultural condition 158

Unification cross-cultural condition 158

Variation cross-cultural condition 159

6.2. Cross-cultural intelligence (XCQ) 161

Nature of XCQ 163

6.3. XCQ amid intricate cultural webs: a grounded theory 164

6.4. Cross-cultural heuristic $r$

Steps in cross-cultural heuristics $\quad 169$

Invoke negative capability to form an open mind 169

Survey the situation to form an understanding $\quad 172$

Set situational anchorage to establish a benchmark $\quad 175$

Apply instrument as the mechanism to reach desired resolution 176

$\begin{array}{ll}\text { Notes on cross-cultural heuristic } & 179\end{array}$

6.5. XCQ within the CQ context 179

Variant perspectives of CQ: mutuality and complementarity 179

Conceptualization $\quad 181$

Methodology 183

Implication 184

Case ZIA

Unveiling a cultural chameleon

7.1. Dutch seed stumbling on Thai soil 190

A new adopted Asian parent $\quad 191$

An evolving identity 192

The Actors $\quad 193$

Founder: Dhr. Adriaan Mannen (Khun Adriaan) 193 
Regional Director - Asia-Pacific: Dhr. Fons van Beek (Khun Fons) 193

Operation Director: Dhr. Ralf Kellerman (Khun Ralf) 194

Sales Manager, Industrial Project: Dhr. Danny Taridise (Khun Danny) 195

Senior Project Manager: Khun Somphob Raksakul (Khun Somphob) 196

Marketing Executive: Khun Nattha Kanchanasakul (Khun Nicki) 197

$\begin{array}{ll}\text { Supporting cast } & 198\end{array}$

7.2. A cultural chameleon in disguise? 199

The indelible pervasiveness of Adriann 199

We are family. $\quad 200$

Karaoke - anyone? 1201

The 'golden pair' in a 'corner' 202

Dutch at heart, Thai up front 203

OK, you are the choir, we are the conductor 204

And here's the score $\quad 205$

And the chameleon keeps on changing? 206

7.3. Lessons from the cultural chameleon 207

Variant cross-cultural modes within intricate cultural webs 207

Collectively exhaustive but not mutually exclusive 207

Forever proceeding but not linearly progressing 209

Negative capability: how chameleons mesmerize friends and foes 209

$\begin{array}{lr}\text { 7.4. Final words } & 212\end{array}$

Khun Adriaan $\quad 212$

Khun Fons $\quad 212$

Khun Ralf $\quad 212$

Khun Danny $\quad 212$

Khun Somphob $\quad 212$

$\begin{array}{ll}\text { Khun Nicki } & 212\end{array}$

Chapter 8

The road ahead

The journey is the destination.

$\begin{array}{lr}\text { 8.1. Taking stock: contributions } & 217\end{array}$

$\begin{array}{ll}\text { Theoretical contributions } & 217\end{array}$

Intricate cultural webs: a holistic view of culture 218

Beyond clashing: an expanded view of cross-cultural condition 219

Cross-Cultural Intelligence (XCQ): a qualitative view of CQ 220

Cross-cultural heuristic - XCQ in action $\quad 221$

Methodological contributions $\quad 222$

Managerial implications $\quad 224$

8.2. The road ahead: future research $\quad 226$

$\begin{array}{ll}\text { Research themes } & 226\end{array}$

Cultivation - cross-cultural practicing and coaching $\quad 226$

$\begin{array}{ll}\text { Assessment - cross-cultural road test } & 228\end{array}$

Research programs $\quad 229$

Substantiation and extension of current model of XCQ 229

Cultural coaching $\quad 230$

XCQ road test and cultural profiling 231

Advancing qualitative research 1231 
8.3. Back to the future: limitations $\quad 232$

$\begin{array}{ll}\text { Origin and illustration } & 232\end{array}$

Vignette TMF - an accidental pilot case study

Vignette ITV - a stillborn case $\quad 238$

Implications $\quad 243$

The two vignettes - deficiencies turned opportunities $\quad 243$

The indispensible value of validation methods and procedures 244

$\begin{array}{lr}\text { Bibliography } & 247\end{array}$

$\begin{array}{lr}\text { Nederlandse samenvatting } & 263\end{array}$

Summary in English $\quad 269$

$\begin{array}{lr}\text { Author's biography } & 275\end{array}$ 


\section{LIST OF DIAGRAMS}

Diagram 1.1: Overview of theoretical memos: thick descriptive cases and vignettes $\quad 13$

Diagram 2.1: Research paradigm landscape 23

Diagram 2.2: Grounded theory procedures and directives 30

Diagram 2.3: Guiding research questions 33

Diagram 2.4: Comparative sampling procedures: random v. purposeful sampling 34

Diagram 2.5: Sampling schema $\quad 37$

Diagram 2.6: Grounded theory: procedural blueprint 45

Diagram 2.7: Comparative view of 'accuracy' as a desired attribute of good research 56

Diagram 2.8: Comparative evaluative criteria $\quad 59$

Diagram 2.9: Methods and procedures to ensure rigor and relevance in qualitative research 65

Diagram 3.1: ADT fact sheet (As of 2007)

Diagram 4.1: A cultural onion model $\quad 105$

Diagram 4.2: Taxonomy of cultural groups 109

Diagram 4.3: Core meanings: Dutch and Thai cultures 114

Diagram 5.1: CDW fact sheet (As of 2007) $\quad 125$

Diagram 6.1: Taxonomy of cross-cultural condition $\quad 155$

Diagram 6.2: Cross-cultural intelligence (XCQ) amid intricate cultural webs $\quad 165$

Diagram 6.3: Cross-cultural heuristic $\quad 170$

Diagram 6.4: CQ: mutuality and complementarity in the variant perspectives 180

Diagram 7.1: ZIA fact sheet (As of 2008) 189

Diagram 8.1: Managerial implications of XCQ 225 



\section{LIST OF REFLECTIONS}

Reflection 1: Tulips, Aloha and the Thai smiles 2

Reflection 2: Emergence of study title 6

Reflection 3: Journey into the familiar unknown $\quad 18$

Reflection 4: How do we make sense of that?

Reflection 5: Khun Kees - a Dutch who liked coriander roots! $\quad 75$

Reflection 6: Khun Joeri - the axe itself! $\quad 76$

Reflection 7: Khun Pim - the unDutched Dutch? 77

Reflection 8: Khun Maleewan - my sisterhood of Thai women? $\quad 78$

Reflection 9: Metaphorically speaking $\quad 92$

Reflection 10:I am captivated by cats and addicted to quotations $\quad 124$

Reflection 11:Khun Geert - a Dutch 'gentle' man! 129

Reflection 12:Khun Chatoeran - a Thai 'gentle' man? $\quad 130$

Reflection 13:Khun Karel - a CEO and a professor $\quad 131$

Reflection 14:Khun Busara - the gatekeeper 132

Reflection 15:Khun Chaowalit - the conductor 133

Reflection 16:'And how many grapes went into the wine?' 152

Reflection 17: How the chameleon fooled and charmed me 188

Reflection 18: Khun Adriaan - a cultural chameleon 193

Reflection 19:Khun Fons - karaoke and all ... 194

Reflection 20:Khun Ralf - a true engineer 195

Reflection 21:Khun Danny - the unDutchable Belgian 196

Reflection 22:Khun Somphob - mediator or adaptor, or both and more? 197

Reflection 23:Khun Nicki - the face of things to come? 198

Reflection 24:Curiouser and curiouser ... 216 



\section{ACKNOWLEDGEMENTS}

NCE UPON A TIME, AND A VERY CONFUSING TIME IT WAS FOR ME, A FORTUNE-TELLER TOLD me that I should count my blessings because for whatever predicament I might find myself in, I would always have someone to support me through thick and thin. Looking back, I do believe she had a point, accidentally, incidentally, or otherwise.

In fact, for this project - which was at times a sort of predicament - I have had not one but countless individuals to thank. And I would bet that this could be the first acknowledgement you read that needs headings, footnotes and even references to structure and formalize it. Given the many individuals I would like to acknowledge, my ingrained Thai view of eternal indebtedness ${ }^{\dagger}$, and my incurable obsession with structure and organization, I beg readers' leniency for a pedantic, yet faithful, tale of my gratitude.

\section{Essentially ...}

I am most grateful to the five Dutch firms and their employees who generously shared with me their experiences and insights, without which this study would never have materialized. The enthusiasm, interest, generosity and sincerity that all my informants gave to this inquiry and in response to my questions and requests made me feel obliged to complete the project with as much enthusiasm and honesty as what they have granted me. Unfortunately, for the need to keep their contributions anonymous, I am unable to thank each and every one of these informants individually here, however much I wish to. Thus, I say, to all my formal informants - I deeply thank you.

I humbly thank the three 'experts' on issues of Dutch and Thai cross-cultural interaction. First is Prof. Sittichoke Varanusantikul who was, at the time of my field work, the Dean of my alma mater faculty - the Liberal Art Faculty of Thammasat University, Thailand. I was introduced to Acharn Sittichoke ${ }^{\ddagger}$ by a long-time friend - Chusak Pattarakulvanit - who teaches at the faculty and knows that Acharn Sittichoke has worked extensively on management training programs for multinationals in Thailand. Acharn Sittichoke provided intriguing observations and insights from a combined perspective of a Thai academic and management consultant, which became valuable in my theorizing. At the other end of the spectrum, I am grateful to Dr. Henry Holmes, the Managing

\footnotetext{
'The notion of 'gratefulness' or 'bunkhun' in the Thai value system is extremely strong. 'Bunkhun' refers to kindness bestowed, for which eternal recognition and reciprocity must be observed (Komin 1991: 139).

' Here I adopt the Thai convention of addressing teachers and professors with the word 'Acharn'. The word 'acharn' literally means 'esteemed teacher' and conveys respect as well as humility.
} 
Director of a management consultancy - Cross-Cultural Management Co., Ltd., for a formal-turned-friendly interview with him. In Thailand, Dr Holmes likely needs no introduction, given his long established work and reputation as the leading expert in the matter of cross-cultural practices with particular emphasis on the Thai culture and its implications to Western expatriates. An academically trained anthropology, Dr. Holmes' thesis work on the Thai culture led him to a life-long personal and professional relationship with Thailand and her people. I learned a lot of intellectual and practical insights that proved to be most valuable to this study from my two hour meeting with Dr. Holmes. He was unusually generous in his sharing of what many would treat as 'trade secrets' and I am particularly touched by this gesture. Last, I was treated with the most memorable Dutch hospitality in Thailand from the Executive Director of the Netherlands Thai Chamber of Commerce (NTCC), Khun Bert Cesar. Prior to our meeting, Khun Bert had already been a key instrument to the very start of this project when he kindly provided me with the list of the NTCC member companies. I used that list to represent the universe of potential cases and start the project. During the field visit, Khun Bert treated me with a friendly Dutch meeting over Thai lunch, in which he related countless anecdotes and practical observations that eventually wove their ways into the ultimate analytical results. I am most grateful to Khun Bert for his personal generosity and professional assistance.

\section{Scholarly ...}

I thank all those who have acted as peer debriefers for this study, either on purpose or by accident. First and foremost are the ultimate peer debriefers - the three members of the beoordelingscommissie - Prof. Mariëlle Heiltjes, Prof. Maarten Verkerk, and Prof. Geert Duyster - who graciously granted me the honour to earn this doctorate based on a mere humble tale. I treasure their careful reading and comments, which will always lend me the sort of unassuming confidence I need to carry on my intellectual curiosity with a good balance of humility and pride.

Throughout this project, I had several opportunities to present and discuss my ongoing analysis and findings at various venues, such as doctoral workshops, research seminars, academic conferences, tutorial sessions with my students, and even casual chit-chats. In all these formal and informal peer review sessions, many fine scholars, colleagues, friends and students have offered their critiques and comments on the interim results, most of which contributed, in one manner or another, to the eventual completion of the project. Here I hope to recount most, if not all, of those unforgettable 'peers' whose input have been invaluable.

In particular, I must thank the key mentors in the Master Class in Global and Cross Cultural Management Research at Aarhus in 2007: Prof. Mikael Søndergaard and Prof. Mark F. Peterson, who continue to be my most inspiring mentors. At the same event, I also had the privilege to learn first-hand from the legendary Prof. Geert Hofstede, whose energy and eloquence have inspired me to be humble yet aspiring in my work. At the same time also, I got acquainted with his son, Prof. Gert-Jan Hofstede, who later 
gave meticulous critiques and suggestions on several key sections in this manuscript, for which I am most grateful. And to all fellow participants at that workshop, I thank you for your friendship and intellect.

UNU-MERIT - and its previous life UNU-INTECH - provided a shelter for my student life for a long period, during which I received invaluable support from many scholars. I thank the three former Deans of the PhD programme - Prof. Shulin Gu, Prof. Sunil Mani, and Prof. Lea Vehlo - for their belief in my potential and their mentoring hand. UNU-MERIT also offered me many venues to present my work, including the annual doctoral workshops supervised by the current Dean Prof. Robin Cowan and two book projects headed by Prof. Geert Duyster. I must thank both these fine scholars and mentors for their interest and respect in students' work and effort. I benefited greatly from having a chance to articulate my study and its preliminary findings in writing and presentation at these workshops and book project meetings. During these events, many experienced scholars made valuable comments on my interim work, which helped me move the project forward. These include Prof. Adam Szirmai, Prof. Wilfred Dolfsma, Dr. Ionara Costa, Prof. Jan Ulijn, and Prof. Nagesh Kumar.

I thank one particular scholar I first got to know outside of academic setting, who has since become both an intellectual and personal inspiration to me. Prof. Peter J. Braspenning was a former mentor of my partner and over time has become our great friend. Besides the many motivating talks of all things under the sun that we always enjoyed after our dinners with Peter, he has shown an unusual kindness to me by helping me with the Dutch text even right in the midst of his own wedding arrangement. Peter has an intriguing mind and most perceptive insight, and I am flattered that he and his wife, Aneliya, now include us in their circle of close friends.

I learned a lot from the many doctoral students who I shared the usual pains and gains of aspiring PhD candidates. I particularly enjoyed my exchange with Ali Syed, Djono Subagio, Franco Bevilacqua, Branka Urem, Semih Akçomak, Abraham Garcia, Yoseph Getachew, Fernando Rodriguez, and Lina Sönne.

Yet, two fellow students in particular have now become my dearest friends. When we first met in Maastricht years ago, Viki Sonntag saw in me something I could not comprehend but made me feel strangely appreciated. The friendship she grants me is fresh and unassuming and her earnest concern for the well-being of humanity always endears me to her and in turn revives my diminishing faith in mankind. Norman Dytianquin and I have come a long way since we first started this PhD ambition together. He has continuously lent me tremendous help and support, personally and professionally. I value his unpretentious scholarship and unaffected take on life. Viki and Norman - thank you for being my friends.

\section{Administratively ...}

I must thank the entire 'behind the scene' people whose work are often undocumented, yet indispensible. 
I thank Corien Gijsberg who welcomed me with cheerful smile and sincere concern the very first day I arrived in Maastricht. Corien was the first Dutch I knew and she made me feel less anxious and more appreciative of the subtle Dutch hospitality that has not been given its credit. I thank Margaret Hoegen, Leann Poeth-Chervenic and the late Jane Williams for their genuine support, respect and interest in PhD students' lives and pursuits. I thank Wilma Coenegrachts for always managing to maintain the subtle balance between pulling, pushing and, at times shoving, us PhD wannabe's forward with her practical attitude and unfailing strength. I thank Ad Notten for always managing to get a hold of the most hard-to-find materials and at the same time showing sincere interest in my research.

To get this manuscript in the deliverable form of a book, I must thank two kind individuals. I thank Jason Rogers for granting me the copyright to use his exquisite photograph of the real spider webs for the cover image. I thank Ruud Leliveld for his sharp eyes and creative critiques on the style and format of this manuscript; I am very grateful for the quality and honesty of his conscientious service.

Lastly, I am most indebted to the administrative and personal support lent me by Evelien in de Braek. I am sure that without her efficient project and people management skills and her lively and kind character, I would have had a much harder time trying to wade my way through this project.

\section{Collegially...}

In the past two and a half years, I have had the privilege to work at the Department of Economics of the Maastricht University School of Business and Economics and it has been such a pleasure. I must thank Prof. Joan Muysken for taking me in and trusting me to do my job with minimal intervention on his part. I am sincerely grateful to Prof. Tom van Veen for having shown me how a great boss can make your professional life as much fun and rewarding as your personal life. I thank him for letting me grow in my job with just the right touch of supervision and a great sense of humour. Tom, I sincerely thank you.

I thank Sylvia Beenen for her kind management of my schedule and all other administrative necessities, all the time with genuine respect for my particular circumstances. I thank Fleur Keune and Silvana de Sanctis for always being there to support me in every way with refreshing sense of camaraderie and humour. It would not be an overstatement if I say that without these three "iron ladies" of the AE2, a lot of people would have found themselves tangled in a whole lot of mess, with me right there in the midst of it.

I thank all my fellow 'tutors' for their comradeship. Particularly, I am grateful to work as a team with Vivianna Prochazka and Pomme Theunissen in the two 'Asian courses'. I learned a lot from them and I am glad we are now not mere colleagues but also good friends. 
And of course, I thank all my students at the SBE, UCM and ES. Working with them has helped me sustain my faith in the value of my research and learning. I hope they learned from me as much as I learned from them.

\section{Personally ...}

I have a small, cozy and crazy family to thank, and a few great friends to salute.

I have four elder sisters - Pan, Tui, Poek and Lek, all of whom having such distinct personalities but the same unfailingly love for me. All five of us live so far apart from each other but our relationship is such that if we do not hear from one another, we remain happy because we know automatically that all must be well. Ours is that kind of close relationship that even after a long period of no contact, we can pick up our pace any time we meet as if we were seeing one another every day. I am fortunate to have them as my sisters. They love me despite, not because of, myself and that has always given me the assurance in whatever I do, this project certainly included. My three nieces and nephew - Puimek, Tawan, and Tasha, have also grown up to be my great friends and I am most delighted by the hope symbolize. Other extended family members - my cousins Pi Tam, and Nok and her family - have helped keep me feel loved. I also feel deeply thankful to my niece's nanny, Yummy, who helped me deal with local necessities for the field work in Thailand and was always there to make sure I had everything I needed to work on my project during the visit.

I have so many long time friends who remain an integral part of my life, even though I have few chances to keep in constant contact with most of them. I will only mention their names here, with the one preamble: dear friends, I sincerely cherish your having been a part of my life. They are, Pinthip Ujjin Vanakul (Tym), Phissa Yaemklin (Toy), Prasong Raksincharoensak, Chairat Lock, Chusak Pattarakulvanich (Lek), Rosemary Wild, Tim Hill, Nancy Treer, Beverley Hope, Ralf Neufang, Manoj Potaphon, Paul Graf, Chamaiporn Apikulvanich, Kaek, Jeannette Estes, Marcel van Hees, and Rossitza Rousseva.

\section{Most dearly ...}

The fortune-teller was right on when it comes to the four individuals I dedicate this work to.

I am what I am today because of my two parents. For whatever human flaws they may have had, they more than made up for it in their ridiculous love for their five daughters, who in turn managed to make the family a fine dysfunctional specimen. My father never had the privilege to go to school but strived to teach himself how to read by observing the monks in their study during his early childhood. Yet, I believe he was the only one person I knew who honestly understood the gist of genuine Buddhist thoughts. My mother only finished high school and worked all her life for virtually one boss, the Thai Royal Family. To me, she was an unassuming feminist who's proved her worth by taking real action with one single purpose - making sure her daughters could 
thrive in any circumstance as well as men, or preferably even better. Both my father and mother had high ambition for their daughters and really dedicated their lives to make ours much easier than theirs. I in turn love them most dearly and am fiercely thankful for their existence in this world. $P a$, wherever you are up there, this one is for you - to me, you are the unanointed PhD. Ma, I know you cannot read this, but I will make sure you know this is also for you and your insane love for me.

When I first met Prof. J. Friso den Hertog some light years ago, I did not realize how blessed I was that he agreed to be my promotor/supervisor - sight unseen. I must thank Prof. Luc Soete for his foresight in suggesting that Friso would be the mentor I sought. I was awkward in my first meeting with this rather senior Dutch Professor and it took me a long while to get to appreciate his intellect and character. Friso's single most striking supervisory and humanitarian trait is his ability to make his apprentices naively marvel at their own work and earnestly question it at the same time. I have rarely met with such a gift in any other mentor. As one of his former apprentices said of him, '[Friso] changed my view several times by asking me only one or two questions' (Verkerk 2004: 490). It was Friso's knack of asking the most excruciating question in the most flattering manner that must have motivated many of his students, me included, to keep on. Over the years, I have come to know Friso and his wife, Ruby (who is one of the most intriguing personalities I have met), outside of the academic environment and I consider myself very fortunate that my path have crossed theirs. Whatever you find of value in this work, I can guarantee Friso's fingerprints are on that intellectually, spiritually, or otherwise. For that, I am utterly grateful.

Humbly, I am also blessed to have a life partner - Arnold Vermeer - who is also my best friend. I must first thank his late father and mother - Peter and Jannie Vermeer for bringing him into this world and letting him be himself ( $\mathrm{Pa}$ and $\mathrm{Ma}$, wherever you are, I thank you for giving me Arnold). This whole PhD project literally would not have even started without Arnold's irrational agreement to support me financially when I had exhausted all other funding. In the end, he gave me far more than the mundane monetary support and for that, I will need more than this whole life time to pay back. Besides being the main financier for this project, Arnold also took on the tasks of a ready peer debriefer, devil-advocate, counsellor, reality-checker, computer system administrator, web-master, translator, and a few other unimaginable functions, all unDutchably contributing to keeping me sane and on track. What can I say, Klukkluk, this work is as much yours as it is mine. Here is to us - now we can get a life. And whatever that life will be, I am dearly thankful I will share it with you and our cats.

\section{Finally ...}

THANK ALL READERS OF THIS MANUSCRIPT, PAST, PRESENT AND FUTURE. WITHOUT YOU IN MIND, IT would not have been that much fun to write. 


\section{Chapter 1}

\section{A ROAD LESS TRAVELLED}

\section{'Sawasdee Kha'}

'Sawasdi' betekent 'hallo' of 'goedendag,' maar ook 'welkom' en 'tot ziens.' 'Sawasdi' wordt uitgesproken als 'Sawatdi.'

Sawasdi Kha voor een vrouw; Sawasdi Khap voor een man.

Thaise gebruiken en begrippen Sifaa webpage: http://sifaa.nl/thailand.htm

"Sawasdee" is the word to use for greetings and goodbye. [...] In order to make the sentences sound polite, Thai people usually use ending particles "Khrap" for males and "Kha" for females at the end of the sentences.

Thai Language Lesson

The University of Tennessee's webpage: http://web.utk.edu/ wratchuk/learningthai/mar3.html

2009

. 3334\$sE\&.

Truth walks toward us on the paths of our questions. [...] As soon as you think you have the answer, you have closed the path and may miss vital new information. Wait awhile in the stillness, and do not rush to conclusions, no matter how uncomfortable the unknowing.

Jacgueline Winspear as part of a monologue in her fiction - Maisie Dobbs

2003

$.3334 \rightarrow 88 E$.

I shall be telling this with a sigh

Somewhere ages and ages hence:

Two roads diverged in a wood, and I-

I took the one less traveled by

And that has made all the difference 
WHEN I learned that I received a scholarship to go and study in Maastricht, the Netherlands, I excitedly called my mother to tell her the great news. Over the phone, her very first response was: 'Tulips!'. I recalled fondly her first response to similar news some years earlier when I was to go and study in Honolulu, Hawaii: to that news she exclaimed 'Aloha!'.

My parents had never set foot in another country until they were well over fifty. My father told me he saw an aeroplane flying over his head when he was a young boy and he thought how magnificent it would feel to be flying.

Now, at the beginning of the twenty-first century, their five daughters are living and working in four countries spanning four continents. They are all interacting with people from many different cultures on a daily basis. Four share their lives with non-Thai partners. Their offspring are far from being typical Thais, let alone pure ones. All of them have to learn to 'live with' people who are not Thai and do not always understand the meanings of our myriad versions of 'the Thai smile.'

Tulips, Aloha, the (in)famous Thai smiles - these are all ready images we conjure up on a daily basis when we come across a 'foreigner'. As difficult as it is to interact with other individuals in our daily life, it's even more challenging when those individuals were born and raised in entirely different systems of belief and are used to manners of sensemaking other than our own. But the world still goes round and round. What is there to fuss over 'cross-cultural' encounter?

I was motivated to fuss over 'cross-cultural' encounter when my own tendency to smile at everything put a rift in my personal life. When I first knew my Dutch partner, many a time he would be so 'unDutchably' irritated at my smiles. 'What are you smiling at - it's not funny!' he would say. I kept telling him, we Thai people smile not only at funny things. We have a thousand and one ways of smiling, to express a thousand and one different thoughts and feelings. 'That is not logical,' was his perfectly sensible Dutch verdict. I now learn to moderate my smiles, while he is slowly getting used to reminding himself that I may not be acting insultingly or ridiculously when I smile at nothing funny.

My situation is neither unique nor exceptional. That is the point. I would like to know how people from different cultures interact and learn from each other. I would like to understand the trick to live in a world where lots of people are born in one place and go to live and work in another place across continents. I would like to know how the Dutch cope and thrive in my native place while I am trying to cope and thrive in their native land.

As it turned out, I was on to something. In the course of this study, I have learned to decipher deeper the meanings of tulips, Aloha and the Thai smiles. I have learned to appreciate more meaningfully my mother's deceptively naive responses. Most gratifyingly, I have also found out that there are more than a handful of people out there who may like to listen to what I have learned.

So, here is my humble tale of tulips, Aloha and the 1001 Thai smiles. 
HIS STUdY, IN A SYMBOLIC SENSE, IS ABOUt a DUTCH Who WOULD LIKE to SAY 'SAWASDEE' tO a Thai, about a Thai who would like to understand and respond to the Dutch 'Sawasdee', and about how the two could learn to make the many versions of 'Sawasdee' work. It is intriguing even such a simple act of greeting and salutation can be complicated enough that if you have never had any experience of the Dutch and the Thai cultures, you will most likely have little appreciation of how complicated 'Sawasdee' can be - how awkward it might be for a Dutch not to know how, when and why to say 'Sawasdee Kha' or 'Sawasdee Khrap' to a Thai, and how strange it could be for a Thai to tune in to the peculiar resonance of the Thai-style greeting coming from a Dutch.

Symbolic talk aside, this is a study about cultural intelligence (CQ) in general and cross-cultural intelligence (XCQ) that was practiced at selected Dutch firms in Thailand in particular. ${ }^{1}$

This study adopted a few unconventional choices - a road less travelled, if you may. The interpretive approach that informed the entire research effort is arguably not a mainstream paradigm of inquiry in management and organization studies (Denzin and Lincoln 2005a, Gephart 2004). The chosen methodological thrust - grounded theory, has only recently gained attention within the social science community and yet to invite immediate recognition, let alone undisputed appreciation or accurate understanding (Allan 2003, Allan 2006, Annells 1997a, Becker 1993, Goulding 1998, Haig 1996, Locke 1996, Locke 2001, Rennie 1998, Suddaby 2006, Thomas and James 2006). The qualitative analytical style, although gaining ground, is undoubtedly not as popularly received as its quantitative counterpart (Denzin and Lincoln 2005b, Gephart 2004). The thick descriptive treatment of case research has yet to be readily appreciated (Simons 1996, Stake 1978, Stake 1995, Stake and Trumbull 1982). The central theme of the study - cross-cultural intelligence, is part of a young theoretical construct - cultural intelligence or $C Q$, which attempts a novel fusion of two intellectual traditions: cross-cultural management and organizational learning (Holden 2001, Holden 2002, Ng and Earley 2006). Finally, the very particular platform for this study - DutchThai cross-cultural interaction, is a niche rather than a standard ground for management and organizational research.

Having said that, all the perils and adversities inherent in such choices are fully acknowledged. The study addresses relevant discords and critiques of its chosen unorthodoxy where and when germane. This, I hope, adds to rather than subtracts from its eventual meaning. To extend the analogy borrowed from Frost's oft misinterpreted poem, the road less travelled has proved to be a rewarding one that led to no regretful longing for the road not taken.

\footnotetext{
${ }^{1}$ The distinction between cultural intelligence and cross-cultural intelligence will be made throughout the manuscript, particularly in Chapter 6. As an introductory note, one of the key findings of this study is that $X C Q$ is in essence a distinct and significant sub-set of CQ.
} 
In the final analysis though, this study is but an attempt to see how and why people of different cultural backgrounds and identities learn, or must learn, from one another and from their own experiences, in order to fulfil their individual and mutual pursuits. It is a plain and mundane, yet material and operative curiosity.

\subsection{Theoretical domain and substantive platform}

In a provocative article, Sumantra Ghoshal called for a radical shift of theorizing paradigm in management research, that is, to move away from the mainstream Popperian view of theorizing as a doctrine of 'formalised falsification' (Ghoshal 2005: 81). Naming the alternative theorizing paradigm 'scholarship of common sense', he explained:

Freud's inductive and iterative approach to sense making, often criticized for being ad hoc and unscientific, was scholarship of common sense. So indeed was Darwin's, who too practiced a model of research as the work of a detective, not of an experimenter, who was driven by the passions of an adventurer, not those of mathematician.

(Ghoshal 2005: 81, emphases added).

In his work, Nigel Holden also advocated a radical shift of focus and conceptualization in cross-cultural management research, both to accommodate to fundamental changes in the real world and to infuse the much needed cross-cultural perspective into the discipline of knowledge management (Holden 2001, Holden 2002). One of his criticisms of the current trend in cross-cultural management research was that 'crosscultural management writers generally prefer to regard actors in broadly definable cultural terms and not against the concrete background of organizational functions, real industries and real problems' (Holden 2002: 53, emphases added).

In his very recent critical review of research in organizational learning and knowledge management, J.-C. Spender invoked a radical return to a past, for studies in these two closely related fields. Interestingly, his view of this return to a past echoed the very spirit of Ghoshal's and Nigel's pleas. He suggested,

[T] he future of both knowledge management and organizational learning is actually a return to a past when our theorizing was more robustly grounded in what we now choose to ignore, managers' experiences and morally burdened practices as they apply their imagination to creating organizations.

(Spender 2008: 172, emphases added).

This study aspired to contribute to the above calls for a radical shift in management and organization research. The study looked into cross-cultural intelligence under a paradigm of enquiry and using a methodology that are very much in line with the spirit of the above proposals. Grounded theory is an inductive and iterative theory-building methodology that is robustly grounded in evidence from real experiences and practices, by mandating researchers to do their detective work in the real world of real people against a concrete setting with the aim to make good sense of a targeted phenomenon. This study employed interpretive grounded theory case research to do just that: making sense of cross-cultural intelligence as experienced and practiced by those working for actual Dutch firms operating in Thailand. The study aimed at building a grounded theory on culture and learning in organizations from evidence collected through observations of and open-ended interviews with Dutch and Thai employees 
who worked for five selected Dutch firms in Thailand. The theoretical domain is thus cultural intelligence, or specifically cross-cultural intelligence, and the substantive platform is cross-cultural interaction between Dutch and Thai cultures.

The above summary characterization may sound as if the theoretical domain and substantive platform were clear-cut matters from the beginning, which is far from being the case. As is characteristic of grounded theory research, the exact nature of the topic under investigation proved to be a distinct case of emergence - best illustrated with my personal reflection on the emergence of the study title itself.

\section{The emergence of study title: a moment of reflexivity}

The theorizing process for this study began basically when I first attempted to define the topic of the inquiry. I embarked on this study as a business-cum-economic student interested in the issue of 'cross-border knowledge exchange' - a handy and readily recognized terminology within the two intellectual communities. At the start, I thought I had identified the focus perfectly: I would look at knowledge exchange in a specific cross-cultural setting, that is, the off-shore operation of a group of representative Dutch firms in Thailand. I basked in my premature solace that I had finally reached a good focus for the study, namely the dual concepts of 'cross-cultural' and 'knowledge exchange' while pinning down a comfortable substantive area too. Alas, the comfort proved to be only short-lived.

Starting from the first day of drafting an invitation to be sent to prospective informants, I already had to struggle with the haunting question: 'what exactly am I looking into?' In subsequent communication with the first few informants, I constantly needed to explain, less to them than to myself, that the focus of my inquiry was neither crosscultural interaction nor knowledge exchange but the two 'together'. Yet, how together, I constantly had to figure out at that early stage. How best to fuse these two concepts? That was a question that lingered for some time in the study and the answer to it emerged rather gradually and crystallized only with the solidification of the key theoretical concepts.

The best illustration of the search for and emergence of theorizing solidification was the way the very title of the study kept being revised. The chronology of this transformation may serve to symbolize the transformation and emergence of the theorizing thrust itself. As I constantly revised the research topic to better reflect the labour of theorizing process, I kept revising the study title to better reflect this thematic change. At first I took this as a bad sign but eventually realized that such was natural with theory building from grounded evidence where nothing can be exact until the theorizing process is saturated and completed for a definite purpose, if not for an infinite ambition. Reflection 2 summarizes the successive versions of study title accompanied by brief reflective notes to illustrate how the eventual title turned out to be an important instance of theoretical emergence. 
Reflection 2: Emergence of study title

\begin{tabular}{|c|c|}
\hline Timeline & Title and reflection \\
\hline Jan 2005 & $\begin{array}{l}\text { Knowledge exchange across borders: the meanings and their } \\
\text { significance - a grounded theory case study of selected Dutch } \\
\text { firms' offshore operations in Thailand. } \\
\text { Initial focus was mainly on the 'approach' itself as at that point, the } \\
\text { 'how' was a certainty while the 'what' needed to be relatively open. }\end{array}$ \\
\hline Sep 2005 & $\begin{array}{l}\text { Knowledge exchange across borders: the meanings and their } \\
\text { significance - a study of selected Dutch firms' operations in } \\
\text { Thailand as a distinct platform of cross-cultural knowledge } \\
\text { exchange } \\
\text { The term 'offshore operations' was replaced with 'platform of } \\
\text { cross-cultural knowledge exchange' to denote a change of focus to } \\
\text { behavioural' activities within a firm, which started to emerge from } \\
\text { the evidence. }\end{array}$ \\
\hline Mar 2006 & $\begin{array}{l}\text { Cross-cultural knowledge dynamics: the meanings and their } \\
\text { significance - A study of Dutch firms' operations in Thailand as } \\
\text { a distinct platform of cross-cultural knowledge dynamics } \\
\text { The terms 'knowledge exchange' was replaced with 'knowledge dy- } \\
\text { namics' to signify the whole range of what can happen with regard } \\
\text { to knowledge in a firm. At this point, the analysis was focused more } \\
\text { on 'knowledge and learning' than on 'culture: }\end{array}$ \\
\hline Sep 2006 & $\begin{array}{l}\text { The 'UnDutchables' in the land of } 1001 \text { smiles - Exploring } \\
\text { cross-cultural intelligence at selected Dutch firms in Thailand } \\
\text { A fundamental crystallization and solidification of the study was } \\
\text { taking place as crisper concepts emerged, resulting in a major revi- } \\
\text { sion of the title. The subtitle part reflected what would eventually } \\
\text { emerge as the core category of the grounded theory from the } \\
\text { study. }\end{array}$ \\
\hline May 2007 & $\begin{array}{l}\text { Cross-cultural intelligence amid intricate cultural webs - A } \\
\text { tale of the UnDutchables in the land of } 1001 \text { smiles. } \\
\text { This was established as the final version in which the two central } \\
\text { theoretical concepts of the whole study are at the forefront: the } \\
\text { core categories of cross-cultural intelligence, and the key condition } \\
\text { of the phenomenon under study - intricate cultural webs. The subti- } \\
\text { tle then serves to denote the study substantive area, as well as } \\
\text { symbolize its 'naturalistic' approach. }\end{array}$ \\
\hline
\end{tabular}

Note: Compiled from author's field notes and coding memos.

\section{Formal theoretical domain: cross-cultural intelligence (XCQ)}

The term 'cross-cultural intelligence' eventually emerged as the core theoretical construct for this study. It is an uncommon term coined, out of necessity, during the conceptualization process because none of the popular terms used in the standard 
literature fully captures the essence of the concept in question as it emerged from grounded evidence. To explain, the study started off with the broad theme of knowledge and learning across cultures, often referred to in diverse terms, including crossborder knowledge flow, cross-cultural knowledge transfer, or knowledge exchange across borders. These are concepts related to what transpires with knowledge and learning in a cross-cultural context. Each term captures and implies a certain viewpoint but none actually does so to convey the central theme of this study: knowledge as it emerges and evolves and learning as it is practiced within the context of human interaction in which cultural multiplicity is the salient condition. Each of the available standard terms lacks a totality of the emerging theme - what signifies the gist of knowledge and learning when actors from different cultures interact. During the earlier stage of theorizing, the term 'knowledge dynamics' came the nearest to convey the core research interest. This term was later replaced by the term cross-cultural intelligence or XCQ because XCQ encompasses the essence of what drives culture and learning in the course of human interaction in a cross-cultural context.

Because the term 'cross-cultural' takes on ambiguous and confusing definitions in the literature but serves an imperative choice in this study, the exact meaning of it for the proposed grounded theory and throughout the manuscript must first be justified and established. Traditionally, the term 'cross-cultural' designates mostly its comparative connotation; hence 'cross-cultural studies' typically stand for comparative studies of different cultures, mostly referring to 'national' cultures. This has to do with the history of cross-cultural research in general and cross-cultural management study in particular, in both of which comparative inquires dominated the initial scene (Adler 1983b, Boyacigiller, Kleinberg, Phillips, and Sackmann 2004, Roberts 1970). Recent debate has, however, raised the need to expand the meaning of the term to reflect greater sophistication in the field. For example, Jackson and Aycan's early proposition elaborated extensively on the fine distinction between 'international' and 'crosscultural' research but still resonated with the notion that cross-cultural studies involved mostly, if not exclusively, a comparative element (Jackson and Aycan 2001: 78). Five years later, the same author moved to argue that although '[c]ross cultural theory has developed strength in comparing nations (as the cultural unit of analysis) in terms of broad value dimensions, [...] little attention has been paid to cross cultural interactions or interfaces as the unit of analysis' (Jackson and Aycan 2006: 10, emphases original). They argued further that '[c]ross cultural interactions and interfaces at multiple levels including intercontinental, inter-national, inter-ethnic, inter-group, inter-organizational levels' must be one of the pressing issues for future cross-cultural research (Jackson and Aycan 2006: 11). Findings from this study corroborate their proposition.

Hence, the meaning of the term 'cross-cultural' must expand to designate issues involving different cultures not only from a comparative angel, but also, and most importantly, from an interactive viewpoint - namely at their interface or 'crossing'. Thus, despite the ambiguity of the term 'cross-cultural' in the literature, the subtle implica- 
tion of the word 'cross' in relation to cultural interface serves a crucial distinction in the eventual grounded theory in this study, compelling the need to retain its use. To emphasize, 'cross-cultural' here signifies not only a comparative aspect but also, and even more importantly, an interactive element of a cross-cultural phenomenon.

Given that definition, I coined the term cross-cultural intelligence to refer to a heuristic-based, strategic capability needed for successful cross-cultural interaction, as I detected through analysis of this study's evidence. It denotes the facility or power to exercise cross-cultural interaction to achieve desired and desirable results. The concept of cross-cultural intelligence or XCQ emerged first during the analysis of the evidence, while I analyzed and interpreted the cases and their theoretical meanings. At that point I had no prior awareness of a similar concept that has only recently been conceptualized by a pioneering group of researchers (Academy of Management 2004, Ang and Van Dyne 2008, Ang, Van Dyne, Koh, Ng, Templer, Tay, and Chandrasekar 2007, Earley and Ang 2003, Earley and Mosakowski 2004, Group \& Organization Management 2006, Ng and Earley 2006, Thomas 2006, Thomas, Elron, Stahl, Ekelund, Ravlin, Cerdin, Poelmans, Brislin, Pekerti, Aycan, Maznevski, Au, and Lazarova 2008, Thomas and Inkson 2003, Thomas, Stahl, Ravlin, Poelmans, Pekerti, Maznevski, Lazarova, Elron, Ekelund, Cerdin, Brislin, Aycan, and Au 2008, Triandis 2006). In fact, it was the emergence of the concept of XCQ that led me to survey relevant literature and came across the said stream of research on cultural intelligence or CQ. Henceforth, I consciously incorporated the work on $\mathrm{CQ}$ into my theorizing process as part of theoretical sampling of existing literature - a principle to be explained in Chapter 2. I eventually located XCQ within the young stream of research on cultural intelligence, an issue to be elaborated at length in Section 6.5 of Chapter 6 .

\section{Substantive platform: Dutch-Thai cross-cultural interaction}

Studies which investigated various aspects of firms that operate either globally, internationally or transnationally abound in both management and economic disciplines (Adler 1983a, Bartlett, Ghoshal, and Birkinshaw 2003, Ricks 1985, Schneider and Barsoux 2003, Shenkar 2004, Toyne 1989, Toyne and Nigh 1998, Usunier 1998, Wright 1970, Wright and Ricks 1994). Studies that looked particularly into the cultural aspect of such firms are no less abundant (Adler 1983b, Adler, Doktor, and Redding 1986, Boyacigiller and Adler 1991, Boyacigiller, Kleinberg, Phillips, and Sackmann 2004, Lewis 1996a). A distinct characteristic that the two groups of studies share, as evident in the cited literature, is a persistent imbalance in influence and representation. First, the majority of these studies were conducted by Western, or Western-inclined, scholars whose particular viewpoint is often implicitly assumed to be either universal or superior, or both. Secondly, the platforms chosen for most of these studies concentrated mainly on a handful of popular cultures and countries, with the less popular cultures or countries implicitly submerged as being part of their more standard 'representatives' the US and major Eastern European countries for the West, and China or Japan for the East, by and large. Although this imbalance in influence and representation has re- 
cently started to decrease in its extent, with increasing inclusion of other, non-Western perspectives, and greater variety of cultures and countries in the investigation, it is still a long way before the scale imbalance will level.

Both the Netherlands and Thailand belong to the group of countries that have been sparingly investigated. Granted, studies on each of the two countries as a single platform of investigation are not entirely scarce (Andrews and Chompusri 2001, Atmiyanandana and Lawler 2004, Baker and Hess 2001, Hampden-Turner and Trompenaars 1993, King 1993, Komin 1989, Komin 1991, Lawrence 1991, Rowley 1998, Siengthai and Bechter 2004, Siengthai and Bechter 2005, Sluyterman 2005, van der Horst 1996, Warner 2004), although the venue for dissemination of such studies remains relatively local, or regional, rather than international, particularly in the case of Thailand. A similar observation also applies to popular press materials that deal with the issue of economics, business, management or culture of the two countries (Cooper 2004, Holmes and Tangtongtavy 1997, Klausner 1993, Mulder 2000, Redmond 2002, Segaller 2005, Vossestein 2004, White and Boucke 2006). There exist no doubt a handful practical guide books in the market to aid cross-cultural sojourners who find themselves in the Netherlands or Thailand. These mostly cover the usual basic facts about the country and common layman observations on its culture.

However, what is most striking in both academic and popular work is a paucity of studies or materials that focus on the actual interface between the Dutch and the Thai. Whether or not this particular cross-cultural platform has a unique property that would bear significance, as distinct from the generalized cross-cultural platform of any two nations, is still a question without any valid answer. There exist as yet too few inquiries to warrant a compelling argument either way. This is rather disappointing given a long and vigorous history of the relationship between the two nations. Trade relations between the two countries dated back to 1601, with 1604 as a landmark for establishment of official trade relations (Brummelhuis 1987). At present, there are well over 150 Dutch firms in Thailand, in various sectors. As of 2004, the Netherlands was Thailand's thirteenth largest trading partner, fifth largest foreign investor and top investor of all the European Union countries (Bank of Thailand 2007). It would seem that there must be a real need for valid understanding of cross-cultural interaction between the Dutch and the Thai, particularly in economic and business settings. Yet, at present, there is an acute scarcity of studies that actually took up this particular platform as the centre stage. This study is most likely one of the few such formal studies.

A final note on the choice of platform relates to the view of researcher as a key research instrument, a view particularly significant in interpretive-qualitative study to be expounded in Chapter 2. Specifically, I, as the key inquirer in this study, can rightly claim both interest in and affinity with the chosen platform. As a naturalized Dutch of Thai ethnic background who needs to live, work and understand both cultures and their cross-cultural practices on a continuing basis, I stand to serve as an apt instrument for this particular research platform. 


\subsection{Research strategy}

This study used grounded theory procedure to sample, collect, analyze and reconstruct qualitative evidence that was framed within case study research design. I characterize this research strategy as interpretive grounded theory case research. Chapter 2 elaborates extensively on this chosen research strategy. Here I will only briefly introduce the two main elements of the strategy: interpretive grounded theory and qualitative case research.

\section{Interpretive grounded theory}

Grounded theory, often misleadingly classified as a 'qualitative' methodology, has made a slow entry into economic and management research and is still fighting for its own footing (Douglas 2003a, Douglas 2003b, Locke 2001, Martin and Turner 1986, Partington 2000, Suddaby 2006, Turner 1983). Fundamentally, grounded theory is an inductive theory building methodology that can be and have been applied to both qualitative and quantitative evidence, although the former has received much more abundant application than the latter. This does not necessarily mean that grounded theory as a methodology is reserved only for qualitative evidence and analysis - an issue to be exemplified in Chapter 2. Having said that, grounded theory is exceptionally powerful as a guiding methodology for analyzing qualitative evidence, especially when combined with other methodological genres, such as case study or ethnography. Such is precisely the research design of choice for this study.

First I must point out the 'interpretive' slant in my application of grounded theory. Chapter 2 begins with a discussion of research paradigm - a discussion normally omitted in the majority of research report - to locate and make distinct the paradigm that informed this entire investigation: interpretive paradigm of inquiry. As such, this study departs from the mainstream paradigmatic choice of positivist or post-positivist view of knowledge. Taken the interpretive stance, I view a quest for knowledge as an engagement between the inquirer and the evidence - an exercise that results in one particular interpretation or meaning of the object of inquiry. In short, under this paradigm, an investigation involves an interpretation of reconstructed meaning, and not a discovery of pre-existed truth. Grounded theory so applied serves as a tool for the interpretation and reconstruction of the meaning an inquirer can make of grounded evidence.

Experienced and novice grounded theorists alike would agree that the very first methodological task when one sets out to conduct a grounded theory study is to establish upfront what procedural blueprint to follow. This is not only because of the well-known and on-going 'Glaser v. Strauss' procedural and philosophical divide (Babchuk 1997, Boychuk Duchscher and Morgan 2004, Charmaz 2000, Heath and Cowley 2004, Kelle 2005, Rennie 1998, Robrecht 1995), but also because even within one single methodological stance, there exist numerous interpretations, suggestions, and proposals of how grounded theory could or should be conducted (Annells 1997a, Annells 1997b, Becker 1993, Dey 1999, Locke 1996, Robrecht 1995, Walker and Myrick 
2006). Even so, guidance abounds in the literature and it is perfectly possible to put together what are deemed most compatible with a researcher's own perspective and the nature of the inquiry at hand. With that in mind, I derived an interpretation of grounded theory procedure that met the research style and purpose of this inquiry.

Diagram 2.6 in Chapter 2 depicts a schematic blueprint of grounded theory methodology based on my interpretation, which is informed primarily by a number of original work of the founders of grounded theory (Corbin and Strauss 1990, Glaser 1978, Glaser 1992, Glaser 1998, Glaser and Strauss 1967, Strauss and Corbin 1990, Strauss and Corbin 1994, Strauss and Corbin 1998) as well as further elaboration on the methodology by seasoned grounded theorists (Allan 2006, Babchuk 1997, Charmaz 2000, Chenitz and Swanson 1986, Dey 1999, Douglas 2003a, Douglas 2003b, Haig 1996, Locke 2001, Martin and Turner 1986, Turner 1983, Walker and Myrick 2006). Although this schematic is an integration of the two major 'Glaser v. Strauss' variants, it is general enough to accommodate most procedures, canons or techniques that have been and may be developed by other grounded theorists. Following is an introductory summary of the methodological concepts and principles of grounded theory, viewed under the blueprint in Diagram 2.6.

As stated, grounded theory is an inductive methodology for building theory that is based primarily, if not solely, on grounded evidence (Corbin and Strauss 1990, Dey 1999, Douglas 2003a, Glaser 1978, Glaser 1992, Glaser and Strauss 1967, Locke 2001, Strauss and Corbin 1990, Strauss and Corbin 1998). The single most important output of grounded theory is thus a 'theory' of the particular phenomenon being investigated, while the single most important process is 'theorizing'. There are five major activities in grounded theory process: research questions, theoretical sampling, evidence collection, coding and theorizing. The key principle that underlies the whole inquiry process in grounded theory is the principle of iteration \& concurrence - meaning that the five major activities do not relate to each other in an absolute linear fashion. The five activities and their iterative and concurrent relation are widely recognized by virtually all grounded theorists as the essential procedural components and principles of grounded theory. Disagreements exist more in the fine details of how the activities should be conducted. The procedural approach I adopted accommodates such competing variants through integration rather than segregation: I did not subscribe dogmatically to either the Straussian or the Glasarian traditions but rather mixed and matched elements from both that I found appropriate to my research purpose. Thus, the blueprint in Diagram 2.6 was based on the conviction that all competing variants of grounded theory are equally legitimate, as long as they abide by the fundamental spirit of the methodology - building theory by induction from grounded evidence. The interpretation of grounded theory adopted for this research was, in brief, based on a relatively liberal, not orthodox, view of its spirit and procedures.

Given the liberal stance, the procedural blueprint in Diagram 2.6 embraces all major methodological approaches so far expounded in the literature and consolidates them to form one single approach. Further elaboration on the blueprint is a subject of 
Chapter 2. Suffice to say for introductory purpose that the consolidated interpretation as depicted in Diagram 2.6 served as a procedural roadmap for this study. Detailed accounts of how this study followed the particular roadmap are also narrated in detail in Chapter 2.

\section{Qualitative case research}

I made fine distinction between case (an analytical unit), case study (analysis of a case), and case study research or case research (a research strategy that is based on case study). This distinction is explained further in Chapter 2. Given this distinction, this study employed one particular case research approach: qualitative case research, that is, the evidence and analysis in this study were based on case information that is fundamentally qualitative in nature, involving both verbal and nonverbal messages, recorded through formal and informal documents, interviews, conversations and observations.

For this study, the case is defined precisely as a group of Dutch and Thai employees of a Dutch operated company in Thailand who must work together on a regular basis. This study then investigated five such cases at five different Dutch firms in Thailand, during the period 2005 to 2007. Case research approach in this studies applied case study, or case analysis, to serve three utilities. First, case study lends functional structure to the inquiry, simply by anchoring research process and all its activities to a core analytical unit or the case. Second, case study serves as a theorizing instrument in that the analysis of the cases becomes the integral part of the grounded theorizing process. Last, the eventual reconstruction of the case as thick descriptive narration stands as a 'virtual reality' for reporting and learning: the three thick descriptive cases (Chapter 3 , 5 and 7) and, to a lesser extent, the two vignettes in Chapter 8, constituted theoretical memos for this study by reporting as well as illuminating its core finding - the grounded theory of XCQ.

\subsection{Research results ${ }^{2}$}

In the above fashion, I embedded qualitative case research as part of the entire grounded theory investigating effort and together they yielded two tightly related research results - a comparative thick descriptive narration and a grounded theory on cultural intelligence. Diagram 1.1 outlines a snapshot of the three thick descriptive cases and two vignettes which together served as the formal theoretical memos for the grounded theory. The diagram highlights the contexts and concepts that characterize the cases and their relations to the grounded theory of $X C Q$, which is summarily

\footnotetext{
2 During the course of the study, parts of interim research results - namely selected sections of Case ADT and CDW, as well as earlier versions of certain theoretical concepts in Chapter 4 and 6 - were published as book chapters in two edited books (Kwanjai and den Hertog 2009, Kwanjai and den Hertog 2010). Since then, several sections of these published materials have undergone substantial revision, particularly those pertaining to the theoretical concepts.
} 
depicted in Diagram 6.2 in Chapter 6. In essence, the grounded theory of XCQ is the core finding of this study and it is elaborated in a successive fashion throughout the manuscript, with Chapters 4 and 6 as the main theoretically oriented elucidation.

Diagram 1.1: Overview of theoretical memos: thick descriptive cases and vignettes

\begin{tabular}{|c|c|c|}
\hline Case / vignette & Context & Concept \\
\hline $\begin{array}{l}\text { Case 'ADT': } \\
\text { UnDutching the Un- } \\
\text { Dutchables }\end{array}$ & $\begin{array}{l}\odot \text { fully owned Dutch subsidiary in } \\
\text { Thailand } \\
\odot \text { design and manufacturing of } \\
\text { data communication technolo- } \\
\text { gies } \\
\odot \text { information technology industry }\end{array}$ & $\begin{array}{l}\text { cultural webs of meanings: compet- } \\
\text { ing meanings in cross-cultural inter- } \\
\text { action: } \\
\rightarrow \text { deadline: dead or alive? } \\
\rightarrow \text { mistake: guilt or shame? } \\
\text { elements of cross-cultural heuristic: } \\
\text { unDutching as an element in cross- } \\
\text { cultural encounter } \\
\text { c core meanings: Dutch and Thai }\end{array}$ \\
\hline $\begin{array}{l}\text { Case 'CDW': } \\
\text { Portrait of an } \\
\text { odd-eyed cat }\end{array}$ & $\begin{array}{l}\odot \text { Dutch-Thai joint-venture }(50: 50) \\
\odot \text { consultancy \& project manage- } \\
\text { ment } \\
\odot \text { civil engineering service industry }\end{array}$ & $\begin{array}{l}\text { - cultural crossing } \\
\rightarrow \text { taxonomy of cultural crossing } \\
\text { elements of cross-cultural heuristic: } \\
\text { role of cross-cultural instruments } \\
\text { the possibility and power of simulta- } \\
\text { neous multiple level cultural analysis }\end{array}$ \\
\hline $\begin{array}{l}\text { Case 'ZIA': } \\
\text { Unveiling a cultural } \\
\text { chameleon }\end{array}$ & $\begin{array}{l}\odot \text { fully owned Dutch subsidiary in } \\
\text { Thailand } \\
\odot \text { consultancy \& project manage- } \\
\text { ment } \\
\odot \text { industrial automation industry }\end{array}$ & $\begin{array}{l}\rightarrow \text { cross-cultural heuristic: its practice } \\
\rightarrow \text { benchmark and mechanism } \\
\text { cultural chameleon } \\
\text { XCQ in action: cultivation and as- } \\
\text { sessment of XCQ }\end{array}$ \\
\hline $\begin{array}{l}\text { Vignette 'TMF': } \\
\text { Getting to know you }\end{array}$ & $\begin{array}{l}\odot \text { fully owned Dutch subsidiary in } \\
\text { Thailand } \\
\odot \text { manufacturing of insulation } \\
\text { construction materials } \\
\odot \text { construction industry }\end{array}$ & $\begin{array}{l}\text { clashing (divisional) cross cultural } \\
\text { condition } \\
\text { - de facto pilot case }\end{array}$ \\
\hline $\begin{array}{l}\text { Vignette 'ITV': } \\
\text { Behind closed doors }\end{array}$ & $\begin{array}{l}\odot \text { fully own Dutch subsidiary in } \\
\text { Thailand } \\
\odot \text { development and distribution of } \\
\text { animal healthcare products } \\
\odot \text { chemical industry }\end{array}$ & - a still born case \\
\hline
\end{tabular}

Note: Summarized from the narratives in Chapters 3, 5, 7 and 8.

\subsection{Style and organization of the manuscript}

The reporting of this study intentionally reflects its unconventional disposition as well as methodological and procedural spirit. As a result, the manuscript's style and organization follow no given convention, or to borrow Wolcott's (2002) vibrant portrayal, I strategically chose to avoid the 'The Chapter Two Problem'.

Of course, chapter 2 can be a review of the literature, if that's what you want, or a dissertation committee - or later, your publisher - insists on. Or it can deal with method. Or it can deal with theory. Or you can go for broke and get all three out of the way at once: theory, method, and review of the literature. But chapter 2 does not have to deal with any of 
these. There is no law governing the contents of chapter 2 any more than there is a law that dissertations must be boring. Furthermore, there is considerable risk that diverting attention to these topics will obscure or overshadow what you have to report. Chapter 2 ought to be whatever you as author want it to be - it's your story, your research, and you ought to feel free to develop it in the manner that best allows you to accomplish your purposes.

(Wolcott 2002: 92)

In fact, for a long period the manuscript suffered tremendously from 'The Chapter Two Problem', when conventional prescription for a 'proper' doctoral dissertation governed the writing. The decision to break free from this convention serves as yet another 'emergence' of the whole research process. I thus subscribed wholeheartedly to Wolcott's conviction that the only law that should govern a reporting of any study must be the fundamental purpose and nature of the study itself.

With that liberation from 'The Chapter Two Problem', the manuscript gradually evolved to take up an iterative and concurrent fashion, blending evidence, analysis, reflections and results together, just as they were in the actual course of the investigation. It is also delivered in a relatively unconventional and variable style. The style and organization were designed to reflect the study contents in its delivery format, thereby fusing and reinforcing the two together.

A few points with regards to style are in order. First is a question of voice. Readers may have already noticed that this manuscript employs more than one voice. The choice was dictated by the way I view inquirer's role in this study. As will be discussed in Chapter 2, I, as the inquirer in this study, assumed the role of an informed interpreter, who must acknowledge my own stance, personal as well as philosophical, whenever it had a potential to affect the eventual interpretation. To denote this awareness and alert readers to it, a first person voice was applied to such instances. Also, stark personal reflections appear throughout the manuscript, at the beginning of each chapter as well as within the cases or chapters; these reflexive accounts are indicated as such with the use of formatting tools such as heading, shaded area and typeface. As will be expounded in Chapter 2, the recognition and exploitation of inquirer's self-awareness - or reflexivity - is a validating instrument widely considered paramount for interpretive research and was thus deliberately asserted in the reporting of this study (Ambert, Adler, Adler, and Detzner 1995, Fox-Wolfgramm 1997, Simons 1996, Stake 1995, Stake and Trumbull 1982). Finally, writing styles employed in this manuscript span a range from intellectually formal to vibrantly poetic, as dictated by the variation in its contents, which alternate between scholarly discussion, thick descriptive narration, and reflexive musing. Poetic license was thus employed deliberately where appropriate to drive home the message of naturalistic inquiry.

Finally, the organization of the manuscript is as follows. After this introduction, a chapter follows to first establish the exact nature of the methodological choice and research design - namely interpretive grounded theory and qualitative case study, and discuss the question of rigor and relevance when applied to qualitative-interpretive study such as this. From then onwards, three thick descriptive cases are intertwined with successive elements of the grounded theory on cross-cultural intelligence in a 
sequence of five chapters. In lieu of a conventional separate literature review chapter, elements from relevant literature are instead blended in the theoretical elaboration if and when they are deemed significant, as was the very manner they emerged in the actual theorization process. The final chapter deliberates on the key contributions, implications and limitations of the study in a reflexive and exploratory spirit, asserting that the nature of the investigation and its resulting theory denote more of a life-long learning journey than a finite learned destination.

Q

ND NOW, LET OUR JOURNEY BEGIN. 



\section{Chapter 2}

\section{QUALITATIVE GROUNDED THEORY CASE RESEARCH 'To see the World in a Grain of Sand'}

This too is scholarship, but it yields theory that does not pretend to be scientific laws but merely serves as temporary 'walking sticks' - in Fritz Roethlisberger's (1977) terms - to aid sense making as I go along, to be used only until a better walking stick can be found. And, if the association of scholarship with common sense seems like an oxymoron, it is only because of the extremely restrictive definition of the term scholarship that the pretense of knowledge has straightjacketed us into.

Sumantra Ghoshal 'Bad management theories are destroying good management practices'

2005

\section{$.3334 \rightarrow 888$.}

I've got you out here in the great open spaces where cats are cats.

Don Marquis archy and mehitabel, 'mehitabel has an adventure'

To see a World in a Grain of Sand, And a Heaven in a Wild Flower, Hold Infinity in the palm of your hand And Eternity in an hour. 
SOMEHOW I always look at this study as a sort of journey. Even in the beginning, I felt like I was starting on a kind of expedition, one that would be both intriguing and exhilarating. When I looked back, that feeling was almost like a hunch, it's rather eerie.

As an academic, I was trained initially to be a literary scholar - reading English literature, probing into the meanings of words and texts that related human action and psyche. I practiced the art of deep 'reading', of making sense out of texts and imageries that the texts conjured up.

As a young researcher, I was prepared mostly to look at things from an objective viewpoint, to be scientific in its customary sense. I learned the science of numeric manipulation and statistical analysis.

I had journeyed from one corner to the other far corner in the intellectual landscape and I came to a junction at the start of this expedition of a doctoral research project. From where I stood at that point in time, the main road was of the tradition that valued mathematical sophistication. But the issue I was interested in could lose its profound meanings so easily if stripped down only to numbers, formulas and mathematical models. My former training in interpretive skill gave me an intuition that the scholarship of qualitative interpretation would be a more effective option, if not the most popular among my immediate peers. I decided to take the road less travelled by those I had made recent acquaintance.

And it has been quite a journey. I made my way down the road I thought void of any road sign to guide me, only to find out that I had only to look for signs of different convention altogether. This path had no clear boards with familiar symbols of directions, rules or guidance. Only natural signposts spotted the passage and it was up to my interpretive skill to make use of them. My initial literary training came of use and I forged my way forward.

The road was not well constructed, nor the surface well paved. Yet I was pleasantly surprised to find that I was far from alone; there were plenty fellow travellers along the way. They were just as competent as my other group of academic acquaintances, if only quite different in their style and inclination. Once or twice, I came upon a junction where the highway cut across, and it was tempting to take turn to the high road. Yet I kept to the chosen path. I knew this road was leading somewhere, but the destination was not readily visible. As it turned out, it was the journey that finally defined the destination.

THIS has not been the most comfortable journey I could have made. But it was certainly pleasing and educational. If I were to make it to any kind of destination, I could at least claim to have helped pave the way for other fellow travellers who may have yet to explore the value of the road they have not taken. 
ROUNDED THEORY AND CASE STUDY SHARE ONE PARADOXICAL QUALITY - BOTH ARE AMONG the most appealing research methodologies to adopt, yet the hardest to articulate. The versatile nature of these two inquiry approaches makes them attractive choices, particularly for study with qualitative and exploratory nature. However, the elusiveness of their procedures, principles and implementation make them vulnerable to sceptics and render study contributions at risk of quick dismissal (Allan 2003, Allan 2006, Becker 1993, Fassinger 2005, Goulding 2001, Simons 1996, Stake 1995, Stake 2000, Suddaby 2006, Thomas and James 2006, Urquhart 2002, Yin 2008). The mutual paradox of grounded theory and case study is a blessing as well as burden. Most grounded theorists and case researchers would agree that, just as the practice of these two methodologies could be quite rewarding, the articulation of any actual implementation and its results could be rather taxing.

Even so, I must attempt to relate how interpretive grounded theory was combined with qualitative case research to form the fundamental research strategy for this study. The extreme versatility of both methodologies compels a clear articulation of choices made when this strategy and its individual elements were implemented. In essence, this study is a qualitative grounded theory case research informed by interpretive paradigm of inquiry. Hence, both grounded theory and case study were exercised to support qualitative-interpretive investigation and conceptualization with the ultimate aim of building a grounded theory of culture and learning in organizations base on comparative case analysis - or to see 'a World' of culture and learning from 'a Grain of Sand' of thick descriptive case, to borrow Blake's analogy. Hopefully, this ardent claim will be gradually affirmed along the course of this chapter.

Apparently, the above chosen strategy does not abide by an orthodox aim or a well-known practice in social science in general, or management research in particular. The aim of the study was not to test formal or grand theory deduced by previous scholars and purporting probabilistic generalization, as are common objective and practice of mainstream social scientists in the past century. What I sought instead was to theorize inductively on a universal issue but for a very specific context and purpose, yet still yielding naturalistic generalization insights. Admittedly, the aim and approach chosen are both counter to readily accepted norm.

Still, the unorthodox choice of research strategy was not a result of a wish or need to be unorthodox. The choice was made based solely on its merits: that the devised strategy comprised overall the best methodology, design, and methods, given the nature of the subject-matter and the character of the research curiosity. As such, the selection of an unorthodox path here in no way implies a dismissal of either the validity or value of the orthodox route. It is not a rejection of the favoured approach but a commendation of its complementary counterpart. Finally, my claim for unorthodoxy is actually overrated. As it will become obvious in the following discussion, the path taken here had been well paved by many previous studies, and increasingly taken in recent years, even though still relatively 'less travelled' (Allan 2006, Creswell 1998, 
Creswell and Maietta 2002, Denzin and Lincoln 2005a, Eisenhardt 1989, Fendt and Sachs 2008, Gephart 2004, Goulding 1998, Haig 1996, Kinach 1996, Locke 2001, Martin and Turner 1986, Suddaby 2006). Humbly put, this study has simply chosen to tread on the 'less travelled' yet well paved path.

\subsection{Interpretive grounded theory}

Wading through literature on grounded theory can be quite a challenging experience. There are basically three major strands of discussion in the literature. The first strand focuses on explaining what the methodology is and how (best) it can (or should) be applied, including work discussing grounded theory in relation to paradigmatic issues (such as: Annells 1996, Annells 1997a, Annells 1997b, Charmaz 2000, Corbin and Strauss 1990, Dey 1999, Douglas 2003b, Fassinger 2005, Glaser 1978, Glaser and Strauss 1967, Goulding 1998, Grant and Oswick, Haig 1996, Keddy, Sims, and Stern 1996, Kinach 1996, Locke 1996, Martin and Turner 1986, Partington 2000, Rennie 1998, Robrecht 1995, Strauss and Corbin 1990, Strauss and Corbin 1994, Turner 1983, Walker and Myrick 2006). The second strand revolves around the dramatic 'Glaser v. Strauss (and Corbin)' divide, either in supporting of one particular stance or investigating the merit of such dispute (such as: Annells 1996, Annells 1997a, Annells 1997b, Babchuk 1997, Boychuk Duchscher and Morgan 2004, Glaser 1992, Heath and Cowley 2004, Kelle 2005, Locke 1996, Rennie 1998, Robrecht 1995). The last, recently prolific strand, centres around the debate over whether the methodology actually deserves the increasing popularity it is recently gaining - either in support of the trend, cautioning against it, or deliberating future directions (such as: Allan 2003, Allan 2006, Becker 1993, Bryant 2002, Charmaz 2005, Chenitz and Swanson 1986, Fendt and Sachs 2008, Goulding 2001, Goulding 2002, Suddaby 2006, Thomas and James 2006, Urquhart 2002). These three issues are obviously not mutually exclusive and many writings do discussion them in conjunction.

Given the above state of affairs, all grounded theory researchers are faced with two challenges: first to clearly establish the legitimacy and blueprint of their individual methodological choices, actual procedural interpretation and eventual implementation of the methodology; and second, to convincingly defend the legitimacy of such choices and practices. Thus, at the risk of rehearsing the many methodological explication writings on grounded theory, what follows aims to clarify grounded theory solely in relation to this study.

In short, it was interpretive grounded theory that served as the methodological thrust for this study; hence the following deliberation starts with an introduction of the fundamentals of research paradigm to establish what is meant by 'interpretive'.

\section{Research paradigm}

All research is shaped by the paradigm of inquiry it is rooted in (Crotty 1998, Guba and Lincoln 1994, Lincoln and Guba 2000). The paradigm of choice is often assumed if it is a well-established, mutual paradigm of a particular research community. To date, main- 
stream research in management, as in many other social science disciplines, mostly assume the positivist/post-positivist paradigm of inquiry, and discussion of this underlying assumption is rarely addressed in a study report (Bryman and Bell 2003, Creswell 2003, Goulding 1999, Guba and Lincoln 2005, Knox 2004, Neuman 2003, Schwandt 2000, Tashakkori and Teddlie 2003). However, that assumption simply cannot be taken for the report of this research, as the paradigm of inquiry underpinning the investigation was not positivist/post-positivist. A brief deliberation on research paradigm is thus crucial, both to locate this study in the paradigm landscape and, more importantly, to establish the mandate and appropriateness of the adopted paradigm and research methodology it subsequently informed.

First, it should be pointed out that, contrary to an unfortunate tendency to bind methodology tightly with paradigm, a methodology in fact does not belong to only one single paradigm (Bryman and Bell 2003, Creswell 2003, Crotty 1998, Elster 1983, Goulding 1999, Guba and Lincoln 1994, Guba and Lincoln 2005, Knox 2004, Lincoln and Guba 2000, Neuman 2003, Schultz and Hatch 1996, Schwandt 2000, Teddlie and Tashakkori 2003). That is, a methodology can take different shapes and forms according to the paradigm of inquiry that is underpinning one instance of its adoption. This observation is of utmost important in an evolving methodology where trials and errors still shape its effectiveness and no one paradigmatic influence has yet taken a 'de facto' claim on the methodology (which is the case, for example, with experimental research that is virtually claimed by the post-positivist paradigm). Grounded theory methodology is one such evolving methodology that is still being moulded by passionate proponents of various competing paradigms, a condition which underlies the heated controversy surrounding the first strand of literature on grounded theory outlined above.

It is thus doubly crucial that the paradigm of inquiry underpinning this study is clearly located and defined. The purpose of the exercise is first to familiarize readers with the interpretive paradigm given that it is still an atypical world view in mainstream social science. Second and even more importantly, the particular paradigm underlying this study also explains why grounded theory was applied the way it was throughout the study.

\section{Research paradigm landscape: a bird's-eye view}

Diagram 2.1 depicts a version of research paradigm landscape that views competing paradigms as locating along a range of hierarchical dimensions, each with variant convictions and principles that span a spectrum anchored by two most opposing worldviews. The diagram also denotes how this study locates in the various elements of the landscape as explained in the note to the diagram.

Competing paradigms stand apart from each other most starkly at the highest dimension of knowledge stance - the further down in the hierarchy, the less the degree of irreconcilability among competing variants. At the very bottom categories of design $\&$ methodology and method \& technique, the divide then becomes virtually irrelevant, 
reflecting the earlier observation about the unfortunate but common tendency to align methodology too tightly with paradigm, as succinctly articulated in Knox's claim for legitimacy of methodological pluralism (Knox 2004). For comparative purpose, Locke's deliberation on qualitative research paradigms somewhat parallels the paradigm landscape proposed here. Her taxonomy of three major paradigms that govern three distinct qualitative research traditions: modernist, interpretive, and postmodernist (Locke 2001: 7-12) - concurs with the delineation that I adopted for this study. By the same logic, it could be argued that numerous work discussing research paradigms (the research paradigm landscape here included), though not entirely in complete agreement with one another, do share fundamental analytical principles and delineation (Crotty 1998, Guba and Lincoln 1994, Guba and Lincoln 2005, Knox 2004, Lincoln and Guba 2000, Schultz and Hatch 1996, Schwandt 2000).

The highest dimension in the proposed paradigm landscape is that of knowledge stance, which serves as the root from which other hierarchical dimensions stem. By knowledge stance is meant the overall epistemological, ontological and axiological claims on the nature of knowledge, how it can be legitimately established and for what purpose. Distinct knowledge stances are difficult, or even impossible, to reconcile as each must form a unique view of human knowledge and its consequences (Crotty 1998, Guba and Lincoln 2005, Knox 2004). Closely correlated to knowledge stance is paradigm of inquiry, that is, the entire system of beliefs that dictates an investigation. Then, tightly knit to paradigm of enquiry is the view on role of enquirer. These three levels form a group of top tier dimensions which can be tightly aligned as they all deal with philosophical standpoint. To use the widely known stance as an illustration, positivism and post-positivism are invariably informed by objectivism and constantly stipulate that an inquirer must act as a neutral observer. Similar alignments can be applied to the other two groups in the spectrum, as depicted by the vertical lines in the diagram. The dimensions further down from these top tier dimensions, however, are of different nature.

For the middle dimensional category, which include branch of knowledge, mode of sense-making, prime objective of inquiry, and nature of evidence \& analysis, each dimension has a variety of convictions and principles that span the horizontal spectrum; yet each distinct variant is ascribable to more than one competing conviction and principle in the other dimensions of the landscape. At this middle level, however, a strong 'affinity,' to use Knox's expression (Knox 2004), can be detected. For example, it would be extraordinary to find a post-positivist study with an inquirer who assumes a role of ardent advocate and puts social transformation as the prime objective of his or her investigation.

For the very bottom dimensional class of design \& methodology and method \& technique, the distinction within a spectrum indeed virtually disappears. Theoretically at least, if not always practically, the various research designs, methodologies, methods and techniques are all fair candidates for mix and match to serve one study, as 
long as the final application is reconcilable within a paradigm of enquiry adopted for that study.

Diagram 2.1: Research paradigm landscape

\begin{tabular}{|c|c|c|c|c|}
\hline \multicolumn{2}{|r|}{ Dimension } & \multicolumn{3}{|c|}{ Spectrum of conviction and principle } \\
\hline \multirow{3}{*}{$\begin{array}{l}\mathbf{T} \\
\mathbf{0} \\
\mathbf{P}\end{array}$} & knowledge stance & objectivism & constructionism & subjectivism \\
\hline & $\begin{array}{l}\text { representative } \\
\text { paradigm(s) of inquiry }\end{array}$ & $\begin{array}{l}\text { positivism } \\
\text { post-positivism }\end{array}$ & interpretivism & $\begin{array}{l}\text { critical-inquiry } \\
\text { participatory } \\
\text { post-modernism }\end{array}$ \\
\hline & role of inquirer & neutral observer & informed interpreter & ardent advocate \\
\hline \multirow{4}{*}{$\begin{array}{l}M \\
\text { I } \\
D \\
D \\
\text { D } \\
\text { E }\end{array}$} & branch of knowledge & \multicolumn{3}{|c|}{ natural sciences / social sciences / ecstatic studies } \\
\hline & mode of sense-making & \multicolumn{3}{|c|}{ causalal / functional / intentional } \\
\hline & objective of inquiry & \multicolumn{3}{|c|}{ prediction / understanding / transformation } \\
\hline & $\begin{array}{l}\text { nature of } \\
\text { evidence \& analysis }\end{array}$ & \multicolumn{3}{|c|}{ quantitative / mixed / qualitative } \\
\hline & design \& methodology & \multicolumn{3}{|c|}{$\begin{array}{l}\text { simulation, experimental research; survey research; ethnog- } \\
\text { raphy/auto-ethnography, phenomenology, case study, } \\
\text { grounded theory, participative inquiry, action research, } \\
\text { discourse analysis, etc. }\end{array}$} \\
\hline $\begin{array}{c}\text { B } \\
\text { O } \\
\text { T } \\
\mathrm{T} \\
\mathrm{O} \\
\mathrm{M}\end{array}$ & method \& technique & \multicolumn{3}{|c|}{$\begin{array}{l}\text { various methods serving four major research activities (with } \\
\text { examples of well-known methods): } \\
>\quad \text { sampling methods (probability, non-probability), } \\
>\quad \text { evidence collection methods (recording, observation, interview, } \\
\text { questionnaire, document harvesting, focus group, narration, } \\
\quad \text { etc.), } \\
\text { analysis methods (statistical, document analysis, cognitive } \\
\text { mapping, coding \& theorizing, content analysis, conversation } \\
\text { analysis, etc.), } \\
>\quad \text { evaluation methods (statistical, non-statistical, etc.). }\end{array}$} \\
\hline
\end{tabular}

Note: Developed based on a selection of key literature (Crotty 1998, Elster 1983, Guba and Lincoln 1994, Lincoln and Guba 2000, Neuman 2003). Underlines denote solid methodological choices for this study, while broken underlines indicate that elements of such choices were also influential to this study, albeit not in a robust sense.

\section{This study in the paradigm landscape}

With respect to this study, choices were made in each dimension of the above paradigm landscape to inform a deliberate effort aiming at moving research in culture and learning towards a new direction deemed crucial for the advancement of this theme, as put forth in recent debate (Boyacigiller, Kleinberg, Phillips, and Sackmann 2004, Earley 2006, Jackson and Aycan 2001, Jackson and Aycan 2006, Triandis 2001, 
Yeganeh, Su, and Chrysostome 2004, Zhu and Ulijn 2005). This body of literature call for cultural study in organizations and management to, first, explore new approaches other than the mainstream post-positivist-quantitative one that has hitherto dominated the scene; and second, to sharpen research perspective by acknowledging and exploiting indigenous experiences of both the inquirers and informants. I took heed of the above suggestions in the following paradigmatic and methodological choices.

This study adopted the constructionist ${ }^{5}$ knowledge stance following a conviction that it is the one most suited for investigation involving man in general, and culture in particular. My conviction was that, with regards to social reality - the prime subject of this study - a positivist notion of a pre-existing, universally posited meaning only to be uncovered was not applicable. At the same time, a subjectivist desire to impose one reality or meaning on the object of study appeared condescendingly ambitious. By contrast, constructionism sees knowledge as a result of the engagement between the knower and the known object. Knowledge is thus a constructed meaning. Knowledge exists only as a result of a unique engagement that a subject (namely an inquirer) cultivates with a known object. There is thus no universal knowledge. An object can be known and have different meanings to different knowers. Unlike subjectivists, though, constructionists do not regard knowledge as an imposition of meaning by the knower (the subject) on an object, making constructionism not quite the same as simply being subjective.

Further, this study attempted an interpretation of social reality vis-à-vis culture and learning in organizations. Under the interpretive paradigm, I make no further claim than that the study's findings serve as one informed version of that reality. As such, the quality and value of this study are up to the judgment of its audience, although guidelines pertaining to assessing rigor and relevance of interpretive-qualitative study such as this could be established - a subject of subsequent sections. Informed by the constructionist-interpretivist stance, I view researcher as an engaging, informed interpreter. Under this view, a researcher gathers evidence as best he or she can and then construes meanings out of the evidence through theorizing process. A powerful metaphor for this view is that of a researcher acting as a 'detective' (Ghoshal 2005: 81, Johnson 1997: 282). Following this analogy, the primary role of a researcher is to actively engage with evidence. For this reason, all elements that make up a researcher: his or her experiences, capabilities, thoughts, ideas, beliefs, creativity, as well as human biases, frailties and flaws, are inevitably, yet productively, part and parcel of the research process and must be exploited for the benefit of the investigation, rather than screened out or suppressed. As Richardson and St. Pierre put it quite succinctly, for interpretive-qualitative research, the 'researcher - rather than the survey, the

\footnotetext{
${ }^{5}$ I use the term constructionism as an umbrella expression to denote the knowledge stance strictly as defined here, which does not follow Crotty's subtle distinction between the terms constructionism and constructivism (Crotty 1998: 57-58) - a rather fastidious distinction that goes beyond the ambition of my deliberation.
} 
questionnaire, or the census tape - is the "instrument" (Richardson and St. Pierre 2005: 960, italics added). This view of researcher as a key research instrument makes it inevitable that the final product of a research project is very much related to who actually is conducting it and how his or her engagement with the inquiry is carried out. In fact, the exact character of a researcher relative to the inquiry constitutes one criterion for the eventual quality and value of interpretive-qualitative inquiry in general, and grounded theory in particular, as well argued by many scholars (Fassinger 2005, Fendt and Sachs 2008, Goulding 2001, 2006, Suddaby 2006) and will be discussed in relation to the notion of reflexivity later in this chapter.

A good researcher under interpretive view is thus one who is able to 'detect' as much relevant evidence as possible and derives a constructed meaning that offers rich understanding of the phenomenon under detection. Metaphorically speaking then, in this study, I saw myself as an informed interpreter and a 'cross-cultural' detective. As the primary inquirer in this study, I - a naturalized Dutch of ethnic Thai origin - can claim close cultural ties to both these national cultures under study. I thus argue further that this particular background did offer great assistance in enriching the quality of my cross-cultural detection, particularly if the principle of reflexivity was wellobserved as a tool to exploit inevitable biases, again an issue to be revisited in the last section of this chapter.

Moving on to the middle tier dimensions, the first of which, branch of knowledge, is included in the landscape primarily as a matter of practical and historical reference since most researchers operate, or at least are trained, within the framework of distinct academic disciplines. Although there is a possible alignment of branch of knowledge to paradigm of inquiry, this alignment is suggestive at best, confusing at worst. Natural science disciplines, such as physics or chemistry, are strongly aligned to the positivist/post-positivist view point, for example. Beyond that, however, the matter is far from clear-cut. The affinity of a branch of knowledge to a particular paradigm is useful only as a practical guidance when a researcher wants to develop an appropriate communication strategy that takes into account the current paradigm affinity of a research community. For this investigation in particular, the affinity of the management research community, to which the study belongs, has been inclined more towards the positivist/post-positivist paradigm (Bryman and Bell 2003, Ghoshal 2005). That was indeed the main motivation for this lengthy and pedantic discussion on paradigmatic issues, in order to introduce interpretive view as an alternative to the familiar positivist/post-positivist inclination.

Next, the dimension mode of sense-making refers to how one views the mechanism that governs the phenomenon under investigation. The term 'sense-making' is a deliberate choice to establish a distinction from the more popular term 'explanation'. My choice is admittedly influenced by the interpretive paradigm that informed this study. Sense-making designates purely an attempt to 'understand' or, tautologically, to make sense. It implies a lower degree of deterministic confidence than the more common term, explanation. The issue of modes of sense-making touches upon a rigor- 
ous philosophical field of what is commonly referred to as 'theories of explanation' - a subject whose scale and scope is far beyond the boundary of this manuscript. Interested readers are invited to read fundamental work on the topic for more extensive elaboration. For example, Cornwell (2004), Garfinkel (1981), Pitt (1988), and Salmon (1990) provided good general elaboration; Little (1991) discussed theories of explanation in the social sciences, and Scherer (2003) gave an extensive review of modes of explanation specifically in the organization theory research community.

The above body of literature debated the many competing schools of thought on explanation and sense-making, but for the purpose of the paradigmatic issue for this study, the delineation as set out by Elster (1983) and elaborated on in association with economic and management inquiry by Ghoshal (2005) was adopted. This taxonomy is simple but broad enough to cover most varieties of modes of sense-making found in modern-day research. That is, most schools of thought on forms and styles of sensemaking can basically be categorized to fall under one of the three basic modes of causal, functional and intentional. Ghoshal (2005) extended Elster's proposition to argue that the practices of organizational and management study to date have been unduly influenced by the aspiration to achieve scientific status of the natural science, resulting in a bias towards causal and functional mode of sense-making even though these are not the ideal modes for the majority of management and organizational inquiries. The reason for this is because, in his words, 'for a vast range of issues relevant to the study of management [...] human intentions matter [... a]nd, intentions are mental states; so to say that a particular action of an individual was caused by a particular intention is not a causal explanation' (Ghoshal 2005: 79). The theme of this study - culture and learning as they relate to cross-cultural practices in organizations involves mostly actors' intention in cross-cultural interaction, although functional and causal explanation do occasionally play limited roles. Motivated by Ghoshal's appeal, this study looked at intentional mechanism as a primary mode of sense-making, but also acknowledged the other two modes if and when they were in effect.

Closely related to mode of sense-making is prime objective of inquiry, which refers to the underlying purpose of the inquiry and the resulting 'knowledge', or rather 'understanding'. Prime objectives of inquiry can be categorized into three varieties: prediction, understanding or transformation. Many scholars argued that understanding, and not prediction or transformation, lies at the heart of interpretive-qualitative research (Ambert, Adler, Adler, and Detzner 1995, Schwandt 1999). As one such research, this study set out from the beginning only to understand more about culture and learning in organizations. At the start, it was quite certain that this inquiry certainly had no intention of actively transforming any of the actors and their social situations. However, it was not clear at first whether the eventual understanding would lead to any probable predictive power or simply provide a better understanding of the issue. During the course of the study, it became increasingly clear that prediction would be neither desirable nor achievable. The very message of the two key core categories - the intractable nature of intricate cultural webs and the heuristic character of 
cross-cultural intelligence - simply rules out any predictive claim. Thus the prime objective of this study turned out to be simply to understand and shed some light on the issue at hand.

With regard to the nature of evidence and analysis, this study was based on evidence from qualitative case study. It applied grounded theory theorizing procedure to analyze the verbal and non-verbal messages gathered from the field and in the literature in order to re-construct and analyze cases which then served as the main theorizing instrument - a process to be elaborated further in subsequent sections.

Finally, the bottom two dimensions cover familiar issues in all research, and hence will not require lengthy discussion other than to state the followings. This study employed interpretive grounded theory as its main methodological thrust, while using qualitative collective case study as its primary analytical instrument, the details of both are the subjects of subsequent sections. Elements of ethnographic methodology were also utilized, particularly in the treatment of the cases, both in their message and delivery. Also, it must be noted that a specific form of ethnography, that is autoethnography, was also employed, given my personal association with the chosen crosscultural platform and in conjunction with the evaluative procedure of reflexivity (Chang 2008, Ellis and Bochner 2000, Mcllveen 2008, Richardson and St. Pierre 2005, Wall 2006). Finally, the exact methods and techniques used in this study adhered primarily to procedural mandates of grounded theory and evaluative concerns for interpretive-qualitative research - the very subjects of the following sections.

\subsection{Grounded theory: procedures and directives}

Grounded theory, often misguidedly classified simply as a 'qualitative' methodology, was conceived and introduced by two sociologists, Barney Glaser and Anselm Strauss, whose book The Discovery of grounded theory: strategies for qualitative research (Glaser and Strauss 1967) marked the official origin of the methodology. Prior to the Discovery book, Glaser and Strauss published a report of the actual research that inspired the discovery of grounded theory methodology: Awareness of dying (Glaser and Strauss 1965), in which they presented what is now regarded as the very first grounded theory - a theory on death and dying, which foreshadowed the well-known stages of grief model later formalized by Kübler-Ross (1973).

The primary inspiration of Glaser and Strauss' new methodology, which they purposely named 'grounded theory' was, in their own words, 'to address ourselves to the equally important enterprise of how the discovery of theory from data-systematically obtained and analyzed in social research-can be furthered' (Glaser and Strauss 1967: 1 , emphasis original). That the methodology has since been known mostly as a qualitatively partial approach is rather misleading in two unfortunate manners. First, although grounded theory has been conceived, developed, employed and advanced mainly as part of an interpretive-qualitative research effort (Goulding 1998, Suddaby 2006), Glaser and Strauss themselves illustrated that the methodology, even though partial to qualitative analysis, could also be used to analyze quantitative data (Glaser and Strauss 
1967: Chapter VIII). Subsequent development has indeed affirmed that grounded theory could well be adapted to work effectively with quantitative data, or even with study that combines both qualitative evidence and quantitative data (Gephart 2003, Knigge and Cope 2006, Lösch 2006). Second and more importantly, the heavy association of grounded theory with qualitative research considerably dilutes the more significant hallmark of grounded theory - that it stands distinct as a theory generation methodology based on inductive analysis of grounded evidence. Essentially, The Discovery of grounded theory (Glaser and Strauss 1967) was a landmark publication less because it addressed qualitative approach, but more because it reported a breakthrough of a discovery of a novel strategy to inductively discover theory, rather than to test deductively formed theory, which has been the mainstream practice of social research. Alas, the subtle pun on the word 'discovery' in the book title (i.e. discovery of a new methodology which aims at discovery of theory) has never been accorded the significance it bears.

As a methodology 'discovered' in an actual research process that investigated human interaction - its process and meaning - grounded theory offers a powerful tool for study involving any type of social process, to which research in management and organizations undoubtedly belongs. To quote Suddaby's praise of the potential value of grounded theory in management research,

\begin{abstract}
One of the more satisfying elements of conducting research in management is that, in contrast to the physical sciences, social science research looks at how human invention continually generates new ways of interaction and organization. Researchers can best understand those new modes of interacting and organizing by using a methodology that is attentive to issues of interpretation and process and that does not bind one too closely to long-standing assumptions. Fortunately, that's precisely what grounded theory is.
\end{abstract}

(Suddaby 2006: 641)

Still, grounded theory has made a slow entrance into management research and is still fighting for its own footing (Douglas 2003a, Douglas 2003b, Locke 2001, Martin and Turner 1986, Partington 2000, Suddaby 2006, Turner 1983). This has to do with three unfortunate circumstances. First of all, mainstream management research has to date adhered rather steadfastly to the established 'scientific' view of research, aka the positivist/post-positivist paradigm of inquiry (Ghoshal 2005). Indeed, grounded theory made its way into management research community partly by virtue of having some so-called 'scientific' elements in it (Haig 1996). Even so, since grounded theory is an inductive approach, grounded theorists in management research constantly see the need to pedantically 'defend' or at the very least extravagantly 'justify' their chosen unorthodox methodology ${ }^{6}$ - a need which management scholars who adopt popular orthodox methodologies such as experimental or survey research have long done away with. This is a strong indication of the young and still to be firmly established status of grounded theory in management research community.

\footnotetext{
${ }^{6}$ As may already be obvious to readers, I must necessarily plead guilty to the charge,
} 
Second, grounded theory itself still operates on an evolving platform, the communication about which made even more challenging by the extremely tacit nature of the method (Annells 1997a, Locke 1996, Rennie 1998, Robrecht 1995, Suddaby 2006). Despite explicit procedural guidelines that abound in literature, most, if not all, methodological writings on grounded theory can only hope to elucidate a research process that is akin to such common tacit practices as driving, swimming, or dancing; the only way to truly comprehend such practices is through actual exercise. As a highly tacit research procedure, grounded theory challenges both investigators and their audience in any reporting of a grounded theory study. To belabour the analogy, when one attempts to explicate what grounded theory is to an audience who has never conducted it, one can only hope to be as successful as trying to teach someone to drive by merely talking about driving without a chance to let the audience be in the driving seat.

Lastly, experienced and novice grounded theorists alike would agree that the very first methodological task when one sets out to conduct a grounded theory study is to establish upfront what procedural blueprint one is to follow. This need arises not only because of the 'Glaser v. Strauss' procedural and philosophical divide that is wellknown and still on-going (Babchuk 1997, Boychuk Duchscher and Morgan 2004, Charmaz 2000, Kelle 2005, Rennie 1998, Robrecht 1995), but also because even within one single methodological stance, there exist numerous interpretations, suggestions, and proposals of how grounded theory can or should be conducted (Annells 1997a, Annells 1997b, Becker 1993, Dey 1999, Locke 1996, Robrecht 1995, Thomas and James 2006, Walker and Myrick 2006). Challenging as it is, the situation is not so disparage as it may sound, because guidance abounds in the literature and the main task is simply to put together elements deemed most compatible with a researcher's own perspective and the nature of the inquiry he or she sets out to investigate. What follows is thus the interpretation of grounded theory procedure deemed to match the research objective and design of this study.

To address the Glaser v. Strauss challenge, it was resolved that instead of asking which of the two Glaser v. Strauss strands would fit the chosen paradigm of inquiry of a study, it was more the question of which parts of the two methodological strands, and other emerging ones, are compatible with the chosen paradigm. With that in mind, I integrated selected elements from different variants of grounded theory to collectively inform this study and my early discussion on paradigmatic issue provided a framework for my interpretation and application of grounded theory. As a matter of fact, this mixed and match strategy is nothing new. Although many scholars adhere to the general trend of exclusive observance of either of the two Glaserian or Straussian variants, a few seasoned grounded theorists adopt a view that is an exception to this trend - Annells (1997b) and Dey (1999) are two of the most notable ones - and their views substantially informed my application of grounded theory in this study.

Diagram 2.2, outlines the integrative view of the procedures and directives of grounded theory so devised to inform this study. Under this view, there are six major procedural elements in grounded theory research - research questions, theoretical 
sampling, evidence collection, coding, theorizing, and final deliverable. These are termed 'elements' rather than 'steps' to underpin the message of the key twin procedural directives: iteration and concurrent.

Diagram 2.2: Grounded theory procedures and directives

- key directives: iteration \& concurrence

\begin{tabular}{|c|c|c|}
\hline Major elements & Procedural principles & Expected output \\
\hline 1. Research question & $\begin{array}{l}\square \text { define formal theoretical domain } \\
\square \text { select substantive platform }\end{array}$ & $\begin{array}{l}\text { grounded identity of the } \\
\text { inquiry }\end{array}$ \\
\hline 2. Theoretical sampling & \begin{tabular}{|l}
$\square$ \\
sampling driven by theorizing purpose \\
- Glaser's 'substantive - theoretical' \\
- Strauss and Corbin's \\
'open - variational - discriminate' \\
$\rightarrow$ integrative sampling principle
\end{tabular} & $\begin{array}{l}\boldsymbol{A} \text { evidence collection protocol } \\
\rightarrow \text { case/field selection } \\
\rightarrow \text { research theme (con- } \\
\text { cepts/categories) }\end{array}$ \\
\hline 3. Evidence collection & $\begin{array}{l}\nabla \text { collection/field work } \\
\rightarrow \text { collection methods } \\
\rightarrow \text { evidence types \& records } \\
\rightarrow \text { sources }\end{array}$ & $\begin{array}{l}\rightarrow \text { raw evidence } \\
\rightarrow \text { scripts/records } \\
\rightarrow \text { field notes }\end{array}$ \\
\hline 4. Coding & $\begin{array}{l}\square \text { coding procedure } \\
\rightarrow \text { Glaser's 'substantive - theoretical' } \\
\rightarrow \text { Strauss \& Corbin's } \\
\text { 'open - axial - selective' } \\
\rightarrow \text { integrative procedure } \\
\square \text { coding archetype } \\
\rightarrow \text { Glaser's 'coding families' } \\
\rightarrow \text { Strauss \& Corbin's } \\
\text { 'coding paradigm' } \\
\rightarrow \text { integrative meta archetype }\end{array}$ & $\begin{array}{l}\text { codes } \\
\text { emerging concepts } \\
\text { emerging categories }\end{array}$ \\
\hline 5. Theorizing & $\begin{array}{l}\nabla \text { working with codes } \\
\rightarrow \text { deciphering concepts \& categories } \\
\rightarrow \text { conceptualizing properties \& di- } \\
\text { mensions of concepts and catego- } \\
\text { ries } \\
\rightarrow \text { theorizing core categories, their } \\
\quad \text { properties and relations } \\
\square \text { theorizing principles } \\
\rightarrow \text { theoretical sensitivity } \\
\rightarrow \text { constant comparison } \\
\rightarrow \text { saturation }\end{array}$ & $\begin{array}{l}\text { theoretical memos } \\
\text { fluid emerging theory } \\
\rightarrow \text { valid grounded theory } \\
\rightarrow \text { substantive theory } \\
\rightarrow \text { mid-range theory } \\
\rightarrow \text { formal theory }\end{array}$ \\
\hline 6. Final deliverable & $\square$ evaluative concerns & professional reporting \\
\hline
\end{tabular}

Note: Author's interpretation based on selected literature (Corbin and Strauss 1990, Dey 1999, Douglas 2003a, Glaser 1978, Glaser and Strauss 1967, Goulding 1998, Goulding 2002, Locke 2001, Martin and Turner 1986, Strauss and Corbin 1990, Strauss and Corbin 1994, Suddaby 2006, Walker and Myrick 2006). 


\section{Elements, principles and output}

Although the following explication of grounded theory procedures is a specific blueprint derived for this study, it retains all fundamentals in the original proposition made by Glaser and Strauss (Glaser and Strauss 1967) and their subsequent extension (Corbin and Strauss 1990, Glaser 1978, Glaser 1992, Glaser 1998, Strauss and Corbin 1990, Strauss and Corbin 1994, Strauss and Corbin 1998). In addition, this blueprint also resonates with major interpretations of grounded theory for social research in its central disposition, if not in every fine detail (Allan 2006, Babchuk 1997, Dey 1999, Douglas 2003a, Fendt and Sachs 2008, Goulding 2002, Locke 2001, Martin and Turner 1986, Suddaby 2006).

\section{Research question}

To varying degrees, prominent grounded theorists advocate adherence to the hallmark 'inductive' principle, with the implication that pre-existing notions of the issue to be investigated must ideally exert no undue influence on the research process. This is easier said than done, as all grounded theorists would agree. The doctrine lies at the heart of the 'emergence v. forcing' debate (Babchuk 1997, Boychuk Duchscher and Morgan 2004, Glaser 1992, Kelle 2005) and poses a constant challenge to all grounded theorists, especially on the ground. The tension of how to deal with the stark reality of pre-existing knowledge is vivid because our mind is not a slate that can be easily wiped clean, no matter how admirable the ideal of absolute unbiasedness is. My solution to this tension was inspired by Dey's well-regarded distinction between an open mind and an empty head (Dey 1993).

Even at the start of a study, one dilemma always surfaces - how does one embark on a study and form research questions that are not overly influenced by prior awareness of the issue to be investigated? In conventional research, comprehensive review of literature is a must and usually conducted at the onset, prior to research question formulation; in grounded theory, existing literature is best treated as yet another set of evidence, to be 'theoretically sampled' and 'constantly compared' with other evidence in an iterative and concurrent manner (Dey 1993). This was exactly how literature was treated and research questions formed for this. That is, although it is advisably ideal for a grounded theory research to start off with a 'clean slate', meaning without prior preconception of the issue to be investigated, this does not necessary translate into a complete ignorance of the inevitable imprints of our intellectual heritage. To reiterate Dey's insight - one that is most often cited in support of the use of literature as part of evidence and how it should influence a grounded theory investigation,

In short, there is a difference between an open mind and empty head. To analyze data, we need to use accumulated knowledge, not dispense with it. The issue is not whether to use existing knowledge, but how. [...] The danger lies not in having assumptions but in not being aware of them. 
At the very least, it is simply impossibly to embark on a study without some idea of the subject at hand - an interest in a particular topic is already an indicator of the existence of prior notion of the issue. Yet that awareness, in any case, is valuable as a simple guideline for the initial period and a part of evidence in the entire process. The key is to use any prior concept as no more than another piece of evidence, in as much as it may be relevant. As such, for grounded theory research, the straightforward task of reviewing literature takes on a new meaning entirely distinct from the usual comprehensive survey of prior theories and studies which aims at establishing authoritative foundation, as is common in deductively informed research. Rather, it is a delicate act of looking at literature as yet another source of evidence, not as the ultimate authority. For this study, review of literature was, to repeat, another iterative and concurrent process of theoretical sampling, evidence collection, coding and theorizing - the same process applied to evidence from the field. Just like with grounded evidence, prior relevant work were analyzed based on their power to aid the investigation.

With that in mind, I only conducted a cursory review of literature at the beginning of this study, enough to form a broad impression of the chosen research subject and help with formulation of research questions. Unlike with most mainstream theorytesting research, research questions for grounded theory are best left at their most general - the degree of generality is only a matter of preference. As Douglas interpreted, Glaser preferred this to be a matter 'entirely dependent upon the perceptions of actors and researchers' while Strauss and Corbin allowed this to be 'predetermined [...] before entering the research site' (Douglas 2003a: 45). This study took the middle ground and the research questions at the onset of the study served only in the form of guiding research questions, with enough flexibility built in them such that emerging evidence could be easily incorporated. I thus formulated very broad general guiding research questions as reported in Diagram 2.3. I designed these questions to be as general and open as possible while still providing a practical structure to the actual initial field investigation. Thus was the compromise of the purely 'emerging' principle advocated by Glaser (1992) and the allowance for some pre-determined 'initial' concepts granted by Strauss and Corbin (1998).

In grounded theory, once the research questions are sketched, the next task is to define formal theoretical domain - that is, what the general theme of the research is and select appropriate substantive platform or the actual real world situation where the investigation will focus on. Even in identifying the formal domain of this study, the eventual concept itself emerged from grounded evidence, as described earlier in the reflection on the emergence of study title in Chapter 1 . At the start, the formal theoretical domain was broadly defined as 'culture and learning in organizations'. Then, in order to identify a practical investigating filed, a specific substantive platform must be framed. Given my professional and personal interests and background, 'Dutch compa- 
nies in Thailand' were selected as a substantive platform for the study ${ }^{7}$. This then became the grounded identity - a specific field and investigating identification that is the output of the research questions element in grounded theory procedure. Grounded identity serves to move the inquiry on to the next procedural element of theoretical sampling.

\section{Diagram 2.3: Guiding research questions}

\begin{tabular}{|c|c|c|}
\hline & Main questions & Sub questions \\
\hline$\square$ & $\begin{array}{l}\text { What are the factors and } \\
\text { interplay involved in the dy- } \\
\text { namics of culture and learning } \\
\text { among parties from different } \\
\text { nations mutually serving a firm } \\
\text { in its operation outside of the } \\
\text { firm's home country? }\end{array}$ & $\begin{array}{l}\text { Are there dynamics of culture and learning when actors } \\
\text { from different nations work together for a firm's opera- } \\
\text { tion outside of its home country? } \\
>\quad \text { If there are such dynamics, how do they operate and } \\
\text { for what purpose? } \\
>\text { Who are the major parties in the dynamics? } \\
>\text { What is the nature of the dynamics? } \\
>\text { What are the major factors involved in the dynamics? } \\
>\text { What transpire in the dynamics? } \\
>\text { Why do the dynamics transpire as described? }\end{array}$ \\
\hline$\square$ & $\begin{array}{l}\text { What is the significance of the } \\
\text { dynamics of culture and learn- } \\
\text { ing to a firm's operations out- } \\
\text { side of its home country? }\end{array}$ & $\begin{array}{l}\text { What contribute to the effectiveness of the dynamics? } \\
\text { Does the effectiveness of the dynamics affect a firm's } \\
\text { operations? If yes, how and to what extent? If not, why } \\
\text { not? }\end{array}$ \\
\hline
\end{tabular}

Note: Reproduced from research notes.

\section{Theoretical sampling}

Sampling strategy for grounded theory - theoretical sampling - qualifies as yet another paradoxical quality of the methodology: it is a decidedly distinctive principle that is always cited, highly valued, yet difficult to explicate and scantily appreciated, particularly by those unfamiliar with grounded methodology and its unique sampling principle (Coyne 1997, Draucker, Martsolf, Ross, and Rusk 2007, Fassinger 2005, Glaser and Strauss 1967).

Apart from convenience (sometimes called accidental or haphazard) sampling, all sampling procedures follow certain logical or theoretical arguments to ensure a valid set of samples. There exist two major categories of such methodical sampling strategies: (statistically) random sampling and (analytically) purposeful sampling. These two strategies stem from one mutual rationale: in most investigation, only a sampling of evidence, as opposed to the entire body of evidence, is needed to achieve credible research analysis and result. This principle is logical as well as practical: there are methods and techniques, statistical or analytical, to derive a sample set that provides enough data or evidence for meaningful analysis, so the quest to examine an entire

\footnotetext{
${ }^{7}$ To repeat information already stated in Chapter 1, I am a naturalized Dutch citizen of Thai ethnic origin.
} 
body of evidence can be naively ambitious at best, unjustifiably redundant at worst (Miles and Huberman 1994, Patton 2001).

Theoretical sampling is a distinct sub-set of purposeful sampling, which is governed by an entirely different sampling fundamentals from those that govern the most commonly practiced sampling strategy, namely random sampling (Byrne 2001, Kuzel 1999, Merriam 1995, Miles and Huberman 1994, Patton 2001, Sandelowski 1995). Therein lies the root of unfortunate misjudgement of purposeful sampling when random sampling principles are applied to judge the validity of purposeful sampling based on the all-too-familiar fixate on the key validating criteria for random sampling - sample size (Patton 2001: 230). Diagram 2.4 summarizes the two main sampling strategies, ending with the key assertion that, for purposeful sampling, it is sample schema and its attributes, as opposed to sample size in relation to statistical error, that must be used to judge sampling validity.

Diagram 2.4: Comparative sampling procedures: random v. purposeful sampling

\begin{tabular}{|c|c|c|}
\hline & $\begin{array}{l}\text { random sampling } \\
\text { (probability sampling) }\end{array}$ & $\begin{array}{c}\text { purposeful sampling } \\
\text { (non- probability sampling) }\end{array}$ \\
\hline Principle/premise & probability theory & analytical logic \\
\hline Objective & $\begin{array}{l}\text { robust statistical inference } \\
\quad \rightarrow \text { reductionist }\end{array}$ & $\begin{array}{l}\text { rich naturalistic generalization } \\
\quad \rightarrow \text { holistic }\end{array}$ \\
\hline Most suitable for & quantitative data & qualitative evidence \\
\hline Sampling process & step-wised \& pre-determined & iterative \& reflexive \\
\hline Analysis method & statistical $\rightarrow$ theory testing & conceptual $\rightarrow$ theory driven \\
\hline Sample attribute & determined by representativeness & determined by informativeness \\
\hline Sample size & determined by statistical error & determined by information saturation \\
\hline Sampling validity & $\begin{array}{l}\text { Quantity is predominant, because } \\
\text { sample size determines quality of } \\
\text { the sample } \\
\Rightarrow \text { sample size needs to be large } \\
\text { enough to ensure statistical } \\
\text { representativeness, given an } \\
\text { acceptable statistical error }\end{array}$ & $\begin{array}{l}\text { Quality is predominant, because sam- } \\
\text { ple attributes determine quality of the } \\
\text { sample } \\
\Rightarrow \text { sample schema needs to be rea- } \\
\text { sonable to ensure conceptual in- } \\
\text { formativeness, given study } \\
\text { purpose and analytical saturation }\end{array}$ \\
\hline
\end{tabular}

Note: Author's integration based on selected literature (Ayres 2007, Byrne 2001, Coyne 1997, Curtis, Gesler, Smith, and Washburn 2000, Denzin and Lincoln 2003, Lincoln and Guba 1985, Luborsky and Rubinstein 1995, Marshall 1996, Merriam 1995, Miles and Huberman 1994, Morse 1995, Morse 2000, Patton 2001, Sandelowski 1995, Stake and Trumbull 1982).

Of the two sampling strategies, random sampling is by far the better known, fully established, widely practiced and readily recognized. In random sampling, sophisticated statistical principles and techniques abound, all based on probability theory and its supposition that if representative variation of the entire body of evidence is harvested, a statistically reliable analysis can be performed to yield credible result that 
could be generalized to the entire population with acceptable statistical confidence (Fitzner 2007, Kaplan 2004, Levin and Fox 2006). The one single law that ensures statistically representative variation is the law of randomness, meaning, in simple terms, equal chance for all samples to be selected. As such, statistically random sampling is in fact paradoxically not a random act, because the entire sampling process is rigorously determined by statistical law well in advance. In other words, random sampling is a non-random procedure designed to achieve a representative set of random samples. Obviously, random sampling is most suitable for studies that deal with robust quantitative data.

Purposeful sampling, by comparison, is based on a different set of principles and rationale that is still evolving, relatively less known and yet crucial for study that deal with qualitative evidence (although purposeful sampling is often used in quantitative study where random sampling is not possible). In essence, purposeful sampling relies on the power of analytical conceptualization - samples are sought not because of their statistical representativeness, but rather for their conceptual informativeness, meaning having high potential to yield rich information (Miles and Huberman 1994: 27, Patton 2001: 230). As such, for purposeful sampling, a statistically random sample could actually be very undesirable, as Miles and Huberman explained,

[This] is partly because the initial definition of the universe is more limited [...] and partly because social processes have a logic and a coherence that random sampling can reduce to uninterpretable sawdust.

(Miles and Huberman 1994: 27)

Hence, in place of probability theory, samples in purposeful sampling are qualified based on a certain set of purposes vis-à-vis study theme and research design. Analytical analysis, as opposed to statistical law, is the key instrument of purposeful sampling, making it an appropriate sampling method for qualitative studies that deal primarily with non-numerical evidence. At the same time, though, the non-numerical nature of purposeful sampling renders it a difficult, though not impossible, procedure to formalize. Many types of purposeful samples are documented and, although the typology is still evolving relative to that of random sampling, the existing typological proposals, such as those put forth by Kuzel (1999), Miles and Huberman (1994) and Patton (2001) do offer ample guidelines to designing valid sampling strategy for qualitative inquiry.

In the final analysis, how the issue of sampling validity is viewed becomes the most crucial distinction between random and purposeful samplings. Undoubtedly, validity is a combined function of sample size and sample attribute, be that for random or purposeful sampling. Yet, it is the relative role and importance of these two dimensions that distinguish how the validity of the two strategies should be determined. As indicated in Diagram 2.4, whereas sample size is predominant in random sampling (sample size relates directly to the required statistical confidence, which in turn, ensures statistical validity), sample attribute is predominant in purposeful sampling because it is a true indication of whether useful and rich information can be harvested. In random sampling, sample size is a clear-cut matter, since the $N$ is easily identifiable when we deal with a well-defined numerical data. Put simply, in random sampling, large sample 
size is often required, as dictated by probability theory. In purposeful sampling, by contrast, sample size is in fact neither clearly defined nor supremely important. This is because we cannot count information in numerical unit like we can count quantitative data, and, more importantly, because the sheer quantity of information is a poor indicator for its eventual quality or usefulness.

For a study that used purposeful sampling like this one, for example, how do we define what the $N$ is, if sample size were to be an issue? This study dealt with three thick-descriptive cases, two vignettes, spanning more than five decades and about twelve locations, with over twenty-five formal informants giving over thirty unstructured and intensive interviews and numerous informal and accidental informants volunteering several observations, altogether relating dozens of critical incidents which involved countless actors. All these parameters by themselves and in combination do reveal information. What then is the $N$ : 3 cases plus 2 vignettes, 50 years, 12 locations, 25 plus informants, 30 formal interviews, large number of incidents, or countless actors? Patton (2001: 242-246) discussed the ambiguity of sample size (or the $N$ ) in qualitative research and gave an insightful answer to similar such questions that are often asked of qualitative study,

My universal, certain, and confident reply to these questions is this: "It depends."

There are no rules for sample size in qualitative inquiry. Sample size depends on what you want to know, the purpose of the inquiry, what's at stake, what will be useful, what will have credibility, and what can be done with available time and resources.

(Patton 2001: 245)

Obviously, for study using purposeful sampling of qualitative evidence, a completely different conceptual benchmark is needed to report and assess sampling validity.

Although there is no formally established terminology for such benchmark, a wellregarded body of work (Kuzel 1999, Luborsky and Rubinstein 1995, Marshall 1996, Miles and Huberman 1994, Morse 1995, Morse 2000, Patton 2001, Sandelowski 1995) all recommended that the entire sampling schema be outlined and considered as a whole in a report of purposeful sampling. Such a schema should relate first the main purpose that underlies the sampling design and the selected sampling strategy that best serves that purpose. Next, the schema then relates pertinent sampling parameters given the stated purpose and chosen strategy, in terms of samples' informativeness and eventual size vis-à-vis resource constraints and information saturation. Based on frameworks of sampling parameters suggested by Miles and Huberman, (1994: 28 and 30) and Patton (2001: 130 and 243-244), a sampling schema for this study evolved, as summarized in Diagram 2.5.

The sampling schema in Diagram 2.5 was derived based fundamentally on the logic that the obvious purpose of any grounded theory study is to build theory based on grounded evidence. Having established research questions and grounded identity as elaborated in the previous section, the specific purpose for this study sampling was naturally to gather grounded evidence for theory-building on the theme of culture and learning in organizations, with sample frame that corresponded to the grounded iden- 
tity that was the output in the research question formulation phase. That grounded identity related to Dutch companies in Thailand.

Diagram 2.5: Sampling schema

Sampling purpose: to gather grounded evidence for theory-building on the theme of culture and learning in organizations, with sample frame that match the grounded identity identified in the research questions - Dutch companies in Thailand.

Sampling strategy: combination or mixed purposeful sampling

- strategy 16 in Patton (2001: 242-246)

$\rightarrow$ primary strategy: theoretical sampling

(Glaser 1978, Glaser and Strauss 1967, Strauss and Corbin 1998)

$\rightarrow$ secondary strategy: multiple case sampling

(Eisenhardt 1989, Miles and Huberman 1994: 28-30)

SAMPLING FRAME

\begin{tabular}{|c|c|c|}
\hline Sampling parameters & Conceptual universe & Eventual samples \\
\hline $\begin{array}{l}\text { setting/platform } \\
\text { Dutch companies in Thailand }\end{array}$ & $\begin{array}{l}150 \text { Dutch companies, as at } \\
\text { beginning of study } \\
40 \text { met sampling purpose } \\
8 \text { accepted invitation } \\
5 \text { were investigated }\end{array}$ & $\begin{array}{l}3 \text { thick descriptive cases } \\
2 \text { vignettes }\end{array}$ \\
\hline $\begin{array}{l}\text { sub-setting } \\
\text { groups of Dutch and Thai ac- } \\
\text { tors that interacted regularly } \\
\text { in those companies }\end{array}$ & $\begin{array}{l}\text { all such groups in the } 150 \\
\text { companies in the universe } \\
5 \text { such groups in the sam- } \\
\text { pled companies were in- } \\
\text { vestigated }\end{array}$ & $\begin{array}{l}3 \text { groups in the } 3 \text { thick descrip- } \\
\text { tive cases } \\
2 \text { group in the } 2 \text { vignettes }\end{array}$ \\
\hline $\begin{array}{l}\text { process } \\
\text { relevant cross-cultural interac- } \\
\text { tions }\end{array}$ & $\begin{array}{l}\text { emergent, aided by theo- } \\
\text { retical sampling }\end{array}$ & $\begin{array}{l}\text { all as recorded in research notes } \\
\text { and scripts and analyzed in } \\
\text { theoretical memos }\end{array}$ \\
\hline $\begin{array}{l}\text { event } \\
\text { critical incidents that yield } \\
\text { theoretical value }\end{array}$ & $\begin{array}{l}\text { emergent, aided by theo- } \\
\text { retical sampling }\end{array}$ & $\begin{array}{l}\text { all as recorded in research notes } \\
\text { and scripts and analyzed in } \\
\text { theoretical memos }\end{array}$ \\
\hline $\begin{array}{l}\text { actor } \\
\text { those involved in the critical } \\
\text { incidents }\end{array}$ & $\begin{array}{l}\text { a range of } 2 \text { to } 5 \text { major } \\
\text { actors and } 2 \text { to } 100+\text { minor } \\
\text { actors per incidents, de- } \\
\text { pending on the critical } \\
\text { incidents }\end{array}$ & $\begin{array}{l}\text { all major actors were investi- } \\
\text { gated, most based on first- } \\
\text { hand accounts and a few } \\
\text { based on secondary accounts } \\
\text { some minor actors were investi- } \\
\text { gated, all based on secondary } \\
\text { accounts }\end{array}$ \\
\hline $\begin{array}{l}\text { informant } \\
\text { formal interviewees and in- } \\
\text { formal or accidental infor- } \\
\text { mants }\end{array}$ & $\begin{array}{l}\text { emergent, aided by theo- } \\
\text { retical sampling }\end{array}$ & $\begin{array}{l}\text { formal interviewees } \\
21 \text { from the } 5 \text { companies that } \\
\text { were investigated } \\
3 \text { experts } \\
\text { informal informants } \\
\text { numerous, as emerged }\end{array}$ \\
\hline
\end{tabular}

Note: Author's integration (in iterative and concurrent manner) based on work cited in the diagram in combination with actual research activities. 
Sampling strategy adopted for this study matches the sixteenth category in Patton's catalogue (Patton 2001: 242-246), namely that of 'mixed strategy'. I devised this strategy following the research design of qualitative grounded theory case research that informed the investigation. The primary sampling strategy must undoubtedly be 'theoretical sampling' - the hallmark sampling principle of grounded theory (Coyne 1997, Draucker, Martsolf, Ross, and Rusk 2007, Glaser 1978, Glaser and Strauss 1967, Strauss and Corbin 1990, Strauss and Corbin 1998). As the name indicates, theoretical sampling is a sampling strategy guided by theorizing directives, defined succinctly by Strauss and Corbin as follows,

Theoretical sampling: Data gathering driven by concepts derived from the evolving theory and based on the concept of "making comparisons," whose purpose is to go to places, people, or events that will maximize opportunities to discover variations among concepts and to densify categories in terms of their properties and dimensions.

(Strauss and Corbin 1998: 201)

Although Glaser (1978) and Strauss and Corbin (1998) advised slightly different detailed procedures, as indicated earlier in Diagram 2.2, the two sampling schemas (Glaser's 'substantive - theoretical' and Strauss and Corbin's 'open - variational - discriminate') share essentially the same fundamental principles. These are 1) iteration and concurrent; 2) constant comparison; and 3) theoretical sensitivity and saturation. This means the decisions on what evidence (cases, actors, events) to be further sought and probed and what issues to be investigated must be made on a continuous basis and in conjunction with coding and theorizing process. This also means that evidence collection takes place both in a conventional fashion (through field observations and interviews, for example) and in an analytical mode (through revisiting field notes and scripts as well as constant literature review). The principle of saturation then guides when no further sampling is needed, namely, when a grounded theory is crystallized, any further sampling will show to be marginally valuable and that indicates a sensible end to sampling.

The secondary sampling strategy for this study followed what Miles and Huberman named 'multiple-case sampling' for which the purpose is to look at 'a range of similar and contrasting cases' (Miles and Huberman 1994: 31) in order to serve the constant comparison analysis. Eisenhardt elucidated this process and stated succinctly that for multiple-case sampling, '[w]hile the cases may be chosen randomly, random selection is neither necessary, nor even preferable' because the goal of theoretical sampling for case study research 'is to choose cases which are likely to replicate or extend the emergent theory' (Eisenhardt 1989: 537). Given that thick descriptive cases were the chosen analytical instrument for this study, a small number of cases was a preferred choice, as advised by seasoned scholars (Creswell 1998, Creswell 2002, Miles and Huberman 1994, Sandelowski 1995). Specifically, Sandelowski (1995) and Miles and Huberman (1994) argued that the richer the cases are, the smaller the number of cases should be in a single study, to account for the rich information vis-à-vis the limit of human conceptual capability, thereby avoiding the hazard of information overload. Creswell $(1998,2002)$ provided specific guidelines by stating that for grounded theory 
case research, the number of cases should be between 3 to 5, with interviews of 15 to 30 informants. Notably, the eventual sampling of this study, as suggested by analytical saturation, corroborated all the above recommendation, as outlined in Diagram 2.5.

About 150 Dutch companies that have operations in Thailand were initially identified. This list was compiled in January 2005 from the registry of active members of the Netherlands-Thai Chamber of Commerce (NTCC). ${ }^{8}$ Of these only a little over forty companies were suitable and possible to approach. The remaining hundred or so companies were either too small (one to two man operation) to provide rich evidence, or too costly to involve, given available resources (for example, those having operations in remote parts of Thailand), or both. I then decided that for the initial theoretical sampling and evidence collection, informants should be those who had worked or were still working for the selected forty companies and would be available for a meeting in the Netherlands at the beginning of the study in 2005 - a tactical choice driven by both theoretical and practical reasons. Practically, this profile of informants would narrow the group to those high-level executives who could open doors for subsequent field work in Thailand. At the same time, these high level executives, with their extensive experience supervising cross-cultural teams, were likely to be informants who could give a broad, general view on the subject matter - the most suitable kind of evidence at a starting point of a grounded theory study. Additionally, it was also more resource effective to conduct the initial phase of evidence collection in the Netherlands where the study was based.

E-mail invitations for interviews, outlining the nature and purpose of the study were sent to all forty companies, using the most targeted contact points obtainable (either directly to the targeted informants themselves or to their immediate offices). Followed up phone calls were made when appropriate. A final total of five companies expressed keen interest in the study and agreed to participate in the initial phase, with suitable informants who could be available for interviews in the Netherlands. Three more companies expressed interests in the study but did not have suitable informants for meeting in the Netherlands, whereas the rest of those invited professed to have no interest in participating or did not respond to the invitation altogether. An examination of the general profiles of the five companies that were able and willing to participate revealed a good mix of variation in general characteristics. Together they represented a wide variety of Dutch companies in Thailand in terms of size, history, industry, international orientation and organizational culture. This suggested a potential rich mix of information to ensure comparative coding and theorizing. Thus, the first phase of theoretical sampling eventually proceeded with formal open-ended interviews with seven Dutch executives from the five firms that accepted the invitation.

In the spring of 2005, seven face-to-face interviews with these informants were conducted within a course of six weeks and spanned a good range of the Dutch geog-

\footnotetext{
${ }^{8}$ I hereby gratefully acknowledge NTCC's assistance in graciously granting me access to their database.
} 
raphy, from Leiden to Rotterdam, Den Bosch, Boxmeer and Waalwijk. Each interview lasted between one and a half to three hours and touched upon the general information about the companies, their operations in Thailand and the informants themselves, additional to issues in the guiding research questions. The interviews went on in parallel with other concurrent activities of theoretical sampling, evidence collection, coding and theorizing. All informants consented to audio recording of the interviews, all of which were then transcribed for use in coding and theorizing. Also, after each interview, observations were reflected upon and written out as field notes or research memos, to guide subsequent interviews and the constant comparison theorizing process.

Notably, the fact that these five firms and their seven executives showed great interest in the issue of cross-cultural interaction served well as an indication of the potential richness of the information to be probed. Enthusiastic interest in a subject usually arises either from experiential gratification or frustration. As the study unfolded, all five firms indeed proved to offer rich information for constant comparison theorizing that could reach saturation. Thus, self-selection, an undesirable attribute in random sampling, stands as a tactically useful sampling technique in purposeful sampling. After all, self-selection, by its very nature, implies an undercurrent of purpose. For this study, three of the five firms eventually provided rich enough information to yield three thick-descriptive cases. The other two firms provided adequate information for the two vignettes, but this was not through lack of information but rather because of technical or tactical mishaps (explained through analysis of the vignettes in the concluding chapter of this manuscript). The experience from this study thus confirmed the potential usefulness of self-selected or voluntary sample in purposeful sampling.

As with all grounded theory studies, theoretical sampling did not end at this point in the study. On the contrary, this was but the beginning of a long iterative and concurrent process that continued throughout, and even beyond, the entire investigation. In the end, beside the main sampling parameters of setting/platform, which eventually yielded the eventual samples in the form of cases and vignettes, the study included five other sampling parameters: group, process, event, actor and informant, as outlined in Diagram 2.5. Relevant elaboration on these other samples will be embedded in the following sections when appropriate, to avoid repetition.

\section{Evidence collection}

Evidence collection in grounded theory must intertwine with the whole theoretical sampling, coding and theorizing process. For qualitative grounded theory research, as with most qualitative inquiry, evidence collection takes place primarily during a prolonged engagement in the field, through observation and open-ended interviews. Evidence is thus embedded in both verbal and non-verbal messages and transmitted through both intentional and accidental channels (Denzin and Lincoln 2003, Glaser and Strauss 1967, Miles and Huberman 1994, Silverman 2004). 
This study made use of two types of evidence: grounded evidence, which constituted the primary source, and relevant literature on the theoretical domain and substantive platform, which served as the secondary source.

As already described, the first round of grounded evidence collection commenced with a series of face-to-face interviews with seven Dutch executives from five companies. In the subsequent field work in Thailand that took place in early 2006 and spanned seven weeks, more grounded evidence was collected in a similar though increasingly focussed manner. In total, fourteen more formal face-to-face interviews were conducted with the various informants in four of the five targeted firms, at the firms' Thai locations, which allowed for detailed observation of other non-verbal evidence. Similar to the first phase, these interviews lasted between one to three hours and all were audio recorded, two of which failed due to technical error. Because both failed-to-record interviews involved two Thai informants from the same firm, the evidence from that firm yielded only a vignette (Vignette TMF) instead of a full thickdescriptive case. In addition, the field work in Thailand failed to materialize any actual interview with informants in one of the targeted firms, the account of which made for Vignette ITV.

All interviews from both phases evolved to involve many key actors in critical incidents that provided evidence on cross-cultural practices for emergent theorized concepts. At the same time, numerous 'accidental' informants were discovered during the field work through those casual events such as:

- an encounter with a Thai taxi driver whose remark on a typical Thai view of karma inspired valuable theorizing;

- an unsolicited yet informative critique of Dutch management style by a supermarket cashier who volunteered the information prompted by the inquiry's Dutch credit card during a grocery shopping;

- daily conversation with the inquirer's own family members that touched upon the field experiences;

- intellectual exchange with friends and colleagues who volunteered their viewpoints on the study evidence.

In this fashion, the field work in Thailand turned out to be more than just field visits of the five companies, but involved constant observations of and conversations with a much broader variety of informants in diverse settings.

Thus, as to be expected, the field work actually involved not just evidence collection but also theoretical sampling, coding and theorizing. The most striking tacit lesson learned once the evidence collection began was that, for grounded theory research, at least under the interpretive paradigm, revealing evidence can be anywhere and grounded theorists must be constantly sensitive to that. Evidence can emerge not only from the words uttered by the informants or statements in written documents, but in so many other non-verbal and subtle sources: design and development of web-sites, organization history and evolution, the manner in which informants (or their gatekeepers) receive (or reject) the inquirers, atmosphere of the organizations, non-verbal 
clues and gestures (including even silence and non-action), and even behaviour of other actors in the setting who might not utter one single word. In addition, evidence from accidental informants mentioned above also proved to be useful. This is 'theoretical sensitivity' at its most astounding spirit and the key to this theoretically sensitive detective work lies in the doctrine of constant comparison, which accentuates how intertwined evidence collection and analysis must be in grounded theory study.

The secondary source of evidence - literature review - was also sought right from the start, as earlier described, and evolved in concurrent and iterative manner throughout the study. At the very beginning, some were identified in the interviews, such as practical how-to books on expatriate lives; other were part of what I identified earlier as 'literature as evidence', namely scholastic writings on cross-cultural issues or on the Dutch and the Thai business and national cultures. Eventually, as the theorizing progressed and deepened, theoretical sampling of literature also became more focussed and intensified. When the two key core categories started to take shape, namely 'intricate cultural webs' and 'cross-cultural intelligence', literature on the budding field of cultural intelligence also emerged as the key body of literature to be more deeply 'sampled', or reviewed in conventional syntax. How concepts from existing literature were woven with grounded evidence will be embedded in the various sections of this manuscript, namely Chapter 4, 6 and 8, which together form the elucidation of this study's key finding - a grounded theory of cross-cultural intelligence.

\section{Coding and theorizing}

The next two procedural elements in grounded theory - coding and theorizing - are so closely related to each other that it makes more sense to give an account of them simultaneously. Notably, these two critical elements form the one area of heated disagreement between the Glaserian and Straussian schools (Babchuk 1997, Boychuk Duchscher and Morgan 2004, Fassinger 2005, Glaser 1992, Goulding 2001, Heath and Cowley 2004, Kelle 2005). To cite Douglas's concise characterization of the difference views: 'Glaser [...] prefers an analytical method that is more general in its frame of reference, while Strauss and Corbin opt for a somewhat more structured set of analytical steps' (Douglas 2003a: 45). Here again, this study took the middle ground.

With the audio records of the first phase interviews in hand, together with research notes, memos and other documents (such as company brochures and web sites), coding activity commenced. The treatment of the primary codes - the interview text - needs explicit description. At first, I attempted to follow the Straussian approach and started to transcribe the audio interview records literally. This eventually proved to be rather fruitless and unappealing, especially because this process moved me to anticipate potential difficulties in further theoretical sampling. Particularly, since some of the subsequent informants for the study would be Thais, interviews would have to 
be conducted in the Thai language. ${ }^{9}$ If that was the case, literal translation would be needed to yield literal transcripts required in strict Straussian coding procedure. That was a possible but undesirable task because it would involve an enormous amount of extra resources and open up room for evidence dilution through the process of literal translation. Eventually, I decided to follow Glaser's more general and creative approach to coding and theorizing but still combined it with Strauss and Corbin's precise methods only when applicable and useful.

Hence, in place of literal transcripts, I devised a structured transcribing procedure, in which about ninety percent of the interview text was transcribed, leaving out obviously irrelevant portions (such as greetings, pleasantries and the like). These were then rearranged and grouped into loose topical units, within each interview - an activity which was in essence a coding activity itself. I applied the same method to subsequent interviews, resulting in an eventual thick volume of loosely 'coded' transcripts that served as a basis for case analysis and writing as well as coding and theorizing in the later stage. This transcribed volume also constitutes part of the audit trail in the procedure to ensure rigor and relevance, to be explained shortly.

In addition, the entire coding and theorizing for this study followed an integrated set of guidelines from a selected set of work on qualitative analysis, not only from grounded theory procedural texts but also some other work on analytical methods for interpretive-qualitative research (Corbin and Strauss 1990, Coyne 1997, Denzin and Lincoln 2003, Dey 1993, Dey 1999, Flick, von Kardorff, and Steinke 2004, Glaser 1978, Glaser 1998, Goulding 2002, Miles and Huberman 1994, Silverman 2004, Strauss and Corbin 1998, Suddaby 2006, Swanson 1986, Turner 1983, Walker and Myrick 2006). Most importantly though, all the fundamental principles of coding and theorizing in grounded theory were adhered to and much appreciated. Particularly, the principles of theoretical sensitivity, constant comparison and saturation proved to be most valuable in the field as well as during theorizing process. Without these, subsequent theoretical sampling and evidence collection would have been quite a chaotic, costly and superfluous experience.

Thus, starting from the very first interview, coding and theorizing proceeded in concurrent and iterative fashion. In the beginning, a set of preliminary theoretical memos eventually resulted from constant coding of evidence by virtually revisiting the interviews, via listening to the audio records, reviewing the interviewing experiences from field notes, and re-examining interview transcripts and coding memos. Out of this and other evidence, particularly relevant literature and intellectual discord, concepts started to emerge that paved way to the next theoretical sampling, evidence collec-

\footnotetext{
${ }^{9}$ Thais are generally more comfortable conversing with their fellow countrymen in Thai, partly due to the relatively low English competency among the majority Thai and partly due to cultural influences. Many Thais find it insulting or snobbish to talk in English to a fellow Thai. Since I am by birth a Thai, it was more natural and appealing for me to conduct the interviews in Thai with all Thai informants in this study, even though all of them spoke fluent English.
} 
tion, coding and theorizing. The iteration and concurrence continued further in the same fashion.

During coding and theorizing, Dey's principle of keeping an open mind but not empty head also applied. This was achieved by adhering to a simple guideline that there are basically four options to deal with prior knowledge once it is set against field evidence and emerging concepts. Prior knowledge can be either 1) confirmed by what emerge from grounded evidence; 2) merged with the emergent; 3) let go because lack of grounded evidence to support it; or 4) extended with support from evidence and emerging concepts (den Hertog 2007). Thus, I treated pre-existing notions of all kinds as yet a unique set of evidence that must go through treatment prescribed by grounded theory procedural principles, especially the three core doctrines of theoretical sensitivity, constant comparison, and saturation.

A grounded theory study reaches closure when the emerging theory is 'valid' again another doctrine found to be succinct yet nebulous. How does one know that what one has is a valid theory? When does one stop, with poise and satisfaction? These were the tension many grounded theorists must have also experienced.

My resolution to this tension was to derive a distinction that was inspired by the following passage from a cultural anthropologist Clifford Geertz, '[...] it is not necessary to know everything in order to understand something' (Geertz 1993: 20, emphases added). In this spirit, if the emerging theory has evolved to a calibre that carries coherent internal logic and can be used to make good sense of the grounded experience and other similar real-world phenomena, it has a quality to be valid. Closure is thus a completion of a theory, not a perfection of it. The significance of understanding as a benchmark for rigor and relevance is well expounded by Schwandt (1999, 2000), whose view on understanding critically inspired the stance taken in this study.

Thus, what I needed to reach closure was a coherent understanding and not the 'ultimate truth'. In other words, this study sought to observe an achievable benchmark for completion, not an ideal quest for perfection. Fortunately, such a test has already been suggested in the literature as elaborated in the next section on the question of rigour and value in qualitative inquiry. With this resolution, the study was brought to a satisfactory closure when its key result - a grounded theory of cross-cultural intelligence by the name of 'cross-cultural intelligence amid intricate cultural webs' - became 'valid', based on the evaluative mandates and procedures explicated in Section 2.4. In this fashion, the many interim output of the twin coding and theorizing elements - codes, concepts and categories, and theoretical memos - gradually came together in the eventual crystallization of the grounded theory of cross-cultural intelligence explicated in Chapter 4, 6 and 8 of this manuscript. Of note also is how the three thick descriptive cases turned out to finally serve as the ultimate theoretical memos, a point to be duly elaborated in Section 2.3 on qualitative case research. 


\section{Final deliverable}

Final deliverable of any research is of course a full report of its conception, implementation and findings. This manuscript serves as the main deliverable of this study, although interim reports were delivered, as part of evaluation process, throughout the investigation. Evaluative concerns and their resulting principles provide a framework for this last element of the entire research procedure. These will be expounded in detail in Section 2.4 .

Diagram 2.6: Grounded theory: procedural blueprint

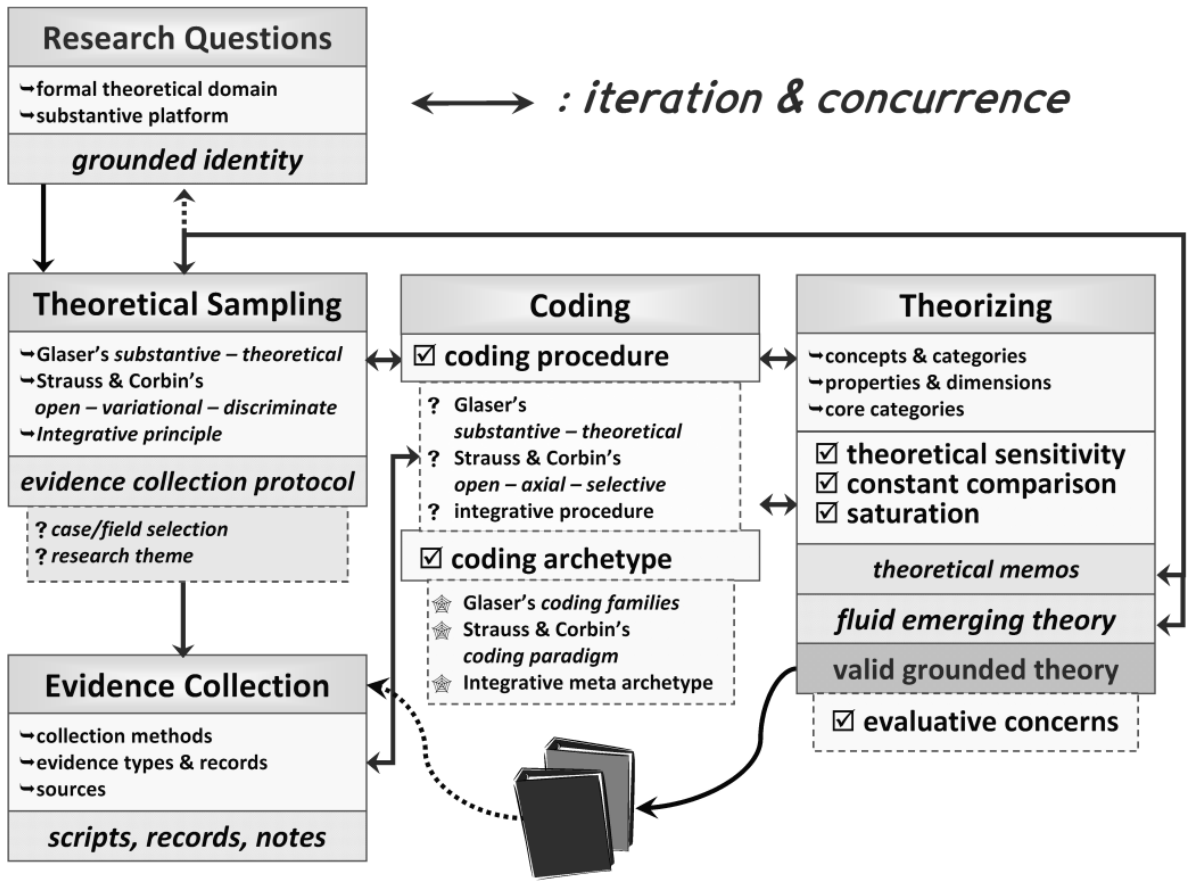

Note: Author's conceptualization based on selected literature (Corbin and Strauss 1990, Douglas 2003a, Glaser 1978, Glaser and Strauss 1967, Locke 2001, Strauss and Corbin 1990, Strauss and Corbin 1994).

\section{Key directives}

By this point, it should have been obvious that grounded theory procedure is characterized most distinctly by its key directives of iteration and concurrence, reflecting the holistic nature of the entire investigative process. In this regards, Diagram 2.2 turns out to be a rather misleading representation of how grounded theory transpires in actual practice, when its elements and characteristics are never carried out or observed in a neatly linear fashion as so presented in Diagram 2.2. The time is now to portray the procedural blueprint of grounded theory, as conceived and practiced throughout this study, in a more true-to-life fashion. Diagram 2.6 depicts such a representation and 
depicts the above accounts on grounded theory procedural mandates in pictorial fashion.

\subsection{Qualitative case research}

Case study research is such an incredibly versatile research strategy that there are practically as many variants of case study research as there are case researchers (David 2006, Feagin, Orum, and Sjoberg 1991, Gerring 2004, Gerring 2007, Gomm, Hammersley, and Foster 2000, Ragin and Becker 1992, Simons 1980, Simons 2009, Stake 1978, Stake 1995, Stake 2000, Yin 2008). As such, the first disclaimer of this section must be that I will now attempt to exemplify the one particular variety of case study research that informed the treatment of cases and vignettes in this study, namely qualitative case research. Specifically, a multiple utility view of qualitative case study lies at the heart of the treatment of cases and the multiple functions they perform in this study.

\section{Case, case study and case study research}

Competing views and definitions regarding case, case study and case study research abound in literature, resulting oftentimes in unnecessary arguments caused more by misalignment of terminologies and definitions rather than true divergence in thematic essence (Becker 1992, Gerring 2004, Verschuren 2003, Yin 1981). To circumvent such misalignment in the reporting of this study, precise definitions of what I mean by 'case', 'case study' and 'case study research' will begin my discussion.

\section{What is a case?}

This question invariably opens most deliberation on case study research (David 2006, Feagin, Orum, and Sjoberg 1991, Gerring 2004, Gerring 2007, Gomm, Hammersley, and Foster 2000, Ragin 1992, Ragin and Becker 1992, Simons 1980, Simons 2009, Stake 1978, Stake 1995, Stake 2000, Yin 2008). In general, most scholars, regardless of how they precisely define the case, view a case as a bounded and holistic instance or unit. A common agreement lies in the combined emphasis on bounded context and holistic treatment, whereas a recurring disagreement concerns the matter of what the instance' or 'unit' could denote - only empirical entity such as a person, a group, an event or incident, and so on, or also inclusive of abstract construct such as a process, a decision, or a policy and so forth (Ragin 1992, Simons 2009: 3-4).

Not to re-invent the wheel, I chose Gerring's succinct definition of a case to be the one that informed this study: a case is a unit or instance which, 'connotes a spatially bounded phenomenon [...] observed at a single point in time or over some delimited period of time' (Gerring 2004: 342). Under this view of the case, a case is thus either an empirical or conceptual unit that is bounded by a specific context - a boundary delimiting basically by space and time and some other conceptual parameters. Such a unit then becomes a 'case' when it is observed as a whole while it operates within its specific boundary. The combined element of holistic treatment and contextual boundary defines a case. 
Under the above interpretation of what a case is, this study's treatment of the case observed the common emphasis on bounded context and holistic treatment while embraced the broad view of a case to include both an empirical entity and an abstract construct, or even a combination of both at the same time. Thus a case can be an empirical unit such as an individual, a team, or a household. It can also be a theoretical construct such as decision making style, interacting process, or nature of interrelationship. Or it can even be a unit that encompasses both parameters simultaneously, such as a decision making style of a specific individual, an interacting process of a particular team, or nature of interrelationship in a certain household. As a matter of fact, the case defined for this study is of the combined type, that is, this study examined the case of cross-cultural interaction of a team of Dutch and Thai employees working for a Dutch firm in Thailand. The investigation observed both the team and their interaction, in a holistic manner and under the specified boundary.

\section{Case study v. case study research}

Another persistent dispute regarding case study research is whether it denotes a concentration on the uniqueness of one singular case or a comparative examination of multiple cases (Dyer and Wilkins 1991, Eisenhardt 1991, Flyvbjerg 2006, Verschuren 2003). To address this dispute and avoid confusion in subsequent discussion, a distinction is made between 'case study' and 'case study research' (to be also referred to in a shorthand manner as simply 'case research'). In a nutshell, case study refers to study of a case whereas case study research denotes a distinct research strategy that employs case study as its central investigating vehicle. Under this distinction, case study involves an examination of a singular case, while case study research can involve case study of one unique case (one case study) or a comparative analysis of several cases (concurrent case studies in one investigation). The decision of whether to employ only one case study or multiple case studies for a particular inquiry is a strategic choice and not an imperative mandate. Case study is an analytical approach; case study research a research strategy.

Thus, I adopted again Gerring's crisp definition of a case study as 'an intensive study of a single unit for the purpose of understanding a larger class of (similar) units' (Gerring 2004: 342, italic original). In other words, a case study is a detailed examination of one particular instance whose claim of uniqueness rests on the specificity of its singular contextual boundary for which no other instance (however similar) can experience. Since the law of physics simply prevents any simultaneous occupation of a single context by more than one entity, it follows that every case must be a unique case, a condition which Simons identified as 'the paradox of case study' - the one property of case study that makes its valuable as an analytical approach (Simons 1996, 2009).

Simons' elucidation of the paradox of case study fundamentally informed the view of case study for this study (Simons 1996). She exemplified her view of a case as a 
unique instance that could paradoxically reflect universal understanding by first citing a passage in the work of MacDonald and Walker,

Case study is the way of the artist, who achieves greatness when, through the portrayal of a single instance locked in time and circumstances, he communicates enduring truths about the human condition. For both the scientist and artist, content and intent emerge in form.

(MacDonald and Walker 1975: 3, cited in Simons 1996: 226).

Thus Simons argued, 'This is the paradox of case study. By studying the uniqueness of the particular, we come to understand the universal' (Simons 1996: 231). She further discussed how, in this sense, a case study is not unlike a work of art, such as a novel, drama or painting. Although such work portray very particular instances of human experience (locked in a singular context), they have the power to convey universal truth that underlies other similar experiences. The 'universal truth' is revealed through an engagement between the observer/reader and the case/story. Similarly, Stake, referring to Dilthey's view of this possibility to extract universal truth from singular experience, explained how case study can be a vehicle to universal understanding: 'we understand ourselves and others only when we transfer our own lived experience into every kind of expression of our own and other people's lives' (Stake 1978, p. 124).

Case study as an analytical approach under the above viewpoint can then be employed as part of a research strategy that must further consider six major strategic considerations regarding the 'case' (the unit) and the 'case study' (the approach) (David 2006, Gerring 2007, Simons 2009, Stake 1995, Yin 2008). The first consideration relates to the nature of the case, that is, what kind case to be taken up for the investigation: extreme or typical case or both. Second is a choice on the number of cases to be investigated: single or multiple case(s) - which in turn influences the third decision on how to analyze the case or cases: as a singular instance or in a comparative manner ${ }^{10}$. The fourth decision determines the nature of the data or evidence to be collected from the case: quantitative data or qualitative evidence, or a combination of both. Fifth is the choice of how to present the analysis of the case data or evidence: as a neutral omnipresent characterization or a naturalistic narration through single or multiple voices. The last deliberation concerns the eventual objectives and results of the entire case study: whether to simply provide description or illustration; to test hypothesized theoretical concept; or to build a theory. Choices made in all these six dimensions in combination constitute one clear research strategy - a particular 'case study research'.

Given the above strategic options, this study can be characterized as a qualitative case research, in the fashion championed by Stake $(1995,2000,2005)$ and Simons (1996, 2009). Briefly, this study sought to investigate multiple cases, each representing either extreme or typical experience, or both (i.e. having both extreme and typical

\footnotetext{
${ }^{10}$ Note that single case study, by default, can only employ detailed analysis of a single case, whereas multiple case study can treat each case as a singular case separately, or in a comparative fashion, or indeed both, which is often the case.
} 
conceptual elements in different aspects), through comparative analysis (or grounded theory constant comparison) of qualitative evidence with theory building as the main objective. The emergent theory and thick descriptive narrations that illuminate it are the eventual results of this entire grounded theory case study research effort. The following section provides details of how I conceived and implemented this chosen qualitative case research strategy.

\section{Qualitative case research: a multiple utility view of case study}

Qualitative case research encompasses the following three key elements: naturalistic essence, mindfulness of complexity, and focus on experiential understanding (Becker 1992, Flyvbjerg 2006, Gerring 2004, Platt 1988, Ragin 1992, Simons 1996, Simons 2009, Stake 1978, Stake 1995, Stake 2000, Stake 2005, Verschuren 2003, Yin 2008). These three properties permeated the way I conceived, analyzed, and reconstructed the cases in this study.

Specifically, I employed the case (the unit) and case study (the analytical approach) to serve three fundamental purposes. Although these three utilities are by no means mutually exclusive, each will be discussed separately to highlight the multiple utility view of case, case study and case study research in this study.

\section{Case study as a structure of the inquiry}

The most practical utility of case and case study is that they provide a handy tool to structure and organize the entire research process, from formulating research questions, sampling, evidence collection, theorizing, and reporting of eventual findings. By having the case as a focal point for all these research activities, the entire investigation can proceed in an orderly yet naturalistic manner, thereby reassuring constant mindfulness of the naturalistic and complex manner of the subject under study. While the 'boundary' of the case helps limit research effort to a manageable and useful scale, the 'holistic' nature of case study renders the investigation a justifiable sense of completion. Simply put, just as laboratory setting and experimental routine or questionnaire and survey design give structure to experimental or survey researchers, the case and case study provide decisive investigative framework and vehicle for case researchers. Metaphorically speaking, the case serves as a scaffold for the inquiry, while the case study provides direction and confidence for case researchers in their manoeuvring through this scaffold.

For this study, the specific case of cross-cultural interaction of a team of Dutch and Thai employees working for a Dutch firm in Thailand made it possible at the start to search for and approach potential ground for the grounded evidence, as well as to frame the initial guiding research questions, as I narrated earlier. The grounded identity of the inquiry was basically rooted in the identity of the case. Then, decisions on who, where, how and what to investigate, observe and interview could be made naturally based on how they related to each particular case. For example, it was quite a simple matter to see that there was no need to talk to all Dutch or all Thai employees 
of the visited firm, since the case as so bounded only demanded evidence from those 'within' the case boundary, or those Dutch and Thai employees who interacted on a regular basis. Also, issues to be investigated or questioned further were determined based on how they might contribute to the matter of cross-cultural interaction. Other more complicated tactical decisions were also made using the same logic. Finally, the case also assisted in the analysis of grounded evidence as well as reporting of research results, to be exemplified in the following discussion.

\section{Case study as a grounded theorizing instrument}

With theory building as the ultimate objective, I intentionally set out to combine case study with grounded theory. Although discussion on how case study can be used to build theory are not lacking (Dooley 2002, Eisenhardt and Graebner 2007, Flyvbjerg 2006, George and Bennett 2005, Gerring 2004, Tripp 1985, Vaughan 1992, Verschuren 2003, Woodside and Wilson 2003), and it is a given that grounded theory is a theory building methodology, little has yet been elaborated on how precisely these two research apparatuses could be combined to aid theory building in practice. This means a study that pair case study with grounded theory needs to find its own tactic for such pairing. My resolution was to construe that case study could actually serve as a powerful grounded theorizing instrument because the process of analyzing and reconstructing the case is itself a rigorous mode of coding and theorizing - a way for researchers to engage themselves with the grounded evidence such that a coherent, deliberate narrative could emerge that also carries theoretical underpinning.

This is very much the same as how Richardson (2003) viewed writing as a mode of inquiry.

Although we usually think about writing as a mode of 'telling' about the social world, writing is not just a mopping-up activity at the end of a research project. Writing is also a way of 'knowing' - a method of discovery and analysis. By writing in different ways, we discover new aspects of our topic and our relationship to it. Form and content are inseparable.

(Richardson 2003, p. 499)

There were several 'writings' in the process of case study in this study. First, following grounded theory procedural mandates, I sketched out numerous 'coding memos', as a way to ponder upon each potential code and concept that emerged during reading (and re-reading) and interpreting (and re-interpreting) of the interview scripts and field notes. Then, some more 'theoretical memos' resulted from the 'within-case' and 'cross-case' analyses (Eisenhardt 1989, Eisenhardt and Graebner 2007, Miles and Huberman 1994, Simons 2009, Yin 2008) to take theorizing process up to the next conceptual level. Finally, the eventual results of the entire case study as a theorizing instrument are the carefully reconstructed cases which serve as the ultimate theoretical memos for this grounded theory study. Each of the three thick descriptive cases thus gradually highlighted the major conceptual categories and their relationship, both within a singular phenomenon of a case as well as in comparison. Readers are invited to observe this point in their reading of the narratives and the intermittent theoretical elaboration that is embedded in them throughout subsequent chapters. 
The story is the message, to mimic McLuhan' famous expression ${ }^{11}$ - which leads naturally to the third utility of the case and case study.

\section{Case study as a 'virtual reality' for reporting and learning}

Cases in this study are presented as a kind of 'virtual reality' woven out of the fabric of grounded evidence harvested from 'the great open spaces' of the field work. I narrated them in naturalistic, ethnographic fashion, inspired by Geertz' concept of 'thick description' and van Maanen's ethnographic view of rich stories as a powerful vehicle to articulate research evidence and theoretical interpretation (Becker 1992, Dyer and Wilkins 1991, Eisenhardt 1991, Geertz 1993, Gerring 2007, Kemmis 1980, Remenyi 2005, Simons 2009, Stake 1995, van Maanen 1979, van Maanen 1988). I would argue that this strategy of reporting cases in the form of vivid story-telling, through multiple lenses and voices of real actors, is a compelling vehicle for 'naturalistic generalization' to be elaborated on in Section 2.4. In brief, the cases so reported serve as a robust conceptual lens that illuminates theoretical concepts they underpin by allowing readers to enter a kind of virtual reality. In such a virtual reality, readers could engage in an experiential exercise of the reality and relate that encounter to their own experience in order to form an understanding of the conceptual interpretation of the case. Three thick-descriptive cases of cross-cultural intelligence in action at three different settings are so narrated in this manuscript. In addition, two vignettes drawn from two other less rich incidents are reported in the concluding chapter as part of final reflexive notes on the entire research process.

I thus intended each thick descriptive case or vignette to be a communication and analytical instrument - a virtual reality - for readers to immerse themselves in. This can serve two purposes. First the cases communicate actual cross-cultural intelligence in action via naturalistic rendering. Second, the cases could also be used as a tool to exercise virtual reality practice, not unlike how cases are used in a class room setting. As a virtual reality, case study maintains the holistic and complex nature of a situation in a form that is compact, comprehensible, naturalistic, engaging and yet manageable. To extend the analogy, a good case, like a good computerized virtual reality environment, induces a realistic engagement such that the observer/reader is able to go through the experience as if he or she were living the very experience, thereby undergoing a virtual 'learning by doing' exercise. Thus, in addition to being a reporting vehicle, the thick descriptive cases could also be used as an experiential learning tool, a concept that will be taken up again in the discussion on future research prospect in the last chapter.

\footnotetext{
${ }^{11}$ I refer of course to McLuhan's well-known expression 'the medium is the message' (McLuhan 1964: 9).
} 


\section{The virtual reality of thick descriptive cases}

In crafting the three major cases, my previous training in literary study exerted obvious influence, particularly in the choice of style for the analysis and delivery. That is, the evidence was analyzed and re-constructed by relying on several standard conventions common in delivery and analysis of a fiction or drama.

Each case begins with a 'hook' - an opening dramatic scene that hints at the main conceptual theme the case illuminates (Naumes and Naumes 2006). As the name implies, the 'hook' is intended to captivate readers' attention and trigger their curiosity, hence dramatic elements are intentionally injected in the hook. Next, the first section of each case sets the scene with description of the actual setting (place and time), giving details of the company itself, both at global and local (Thai) level. The time period of the case involves primarily the time of the field investigation but with flashback and fast-forward as relevant. This first section of each case also relates the characters or actors, detailing the profiles and characterization of those individuals who were involved in the critical incidents narrated in subsequent sections. All major characters are described individually, with each description accompanied by my reflexive note as an inquirer. Supporting casts are the group of employees that provided background for the setting and the story. This first background section is delivered using the 'omnipresent' voice of the investigator/observer.

The second section then narrates the plot and sub-plot, that is, the critical incidents that form the storyline (major and minor) in the case. The incidents are narrated through multiple lenses and voices - through the eyes of those living through the incidents themselves. This section is where the ethnographic thick descriptive style comes into play: this is the 'virtual reality' of the case.

Each case then ends with the third section, where the voice of the investigator/observe now returns to deliberate on the theme of the whole story. This section constitutes the interpretation of what emerged as the main 'abstract concept that is made concrete through the images, characterization, and action' (Meyer 2007) in the case. Thus, what is referred to as the theme in a literary piece becomes the theoretical concept and category that form the elements of the emergent grounded theory. The concluding section of each case ends simply with selected quotations from the key actors, as a final tribute to the informants and their experiences that informed the case and case study.

\section{Convention and poetic license}

For ethical reasons and to preserve the anonymity of the informants and the companies, I made use of the following conventions and poetic license in the narration of the cases.

- Names of the firms: I invented a three letter abbreviation to refer to each company in lieu of its full name. The abbreviation might make sense to the informants but will not be readily obvious to outsiders.

- $\quad$ Names of the characters: also I used fictional names for all characters. 
- Form of address: when first introduced, a Dutch title was used for a Dutch character (thus Dhr. or Merv.) and a Thai formal address (Khun) for a Thai character. In the narrative, all characters were addressed using the Thai 'Khun' followed by their first names, which is the common formal way of addressing people in Thailand. ${ }^{12}$ The choice of this convention followed the fact that the actual settings for all stories were basically in Thailand and this is how people in real life address each other in the Thai business and social context.

- Names of places: remained the same as in real life, although only for general geographic locations with no specific addresses given, to lend naturalistic flavour to the narration.

- Dates: also remained the same as in real life, although only rough dates (such as 'the early 1980 ' $s$ ') rather than exact ones were used in most instances.

- Direct quotations: in the narrative, any direct quotation was indicated with the italic font style.

\section{Factual accuracy}

Although I kept the factual details as accurate as possible, a few minor facts may not be a hundred percent accurate, mostly to keep the story line as uncomplicated as possible without sacrificing analytical content. On the other hand, I took great care to narrate the characters' viewpoints, especially when they were expressed concretely or directly during the interviews, as accurately as possible. However, the stories remained my own interpretation of the facts, given the adopted research paradigm outlined earlier. Reflexive accounts (see explanation below) - embedded throughout the manuscript, serve to underscore this view on the issue of accuracy. This, I hope, should aid readers in their evaluation of my interpretation of the cases.

\subsection{Evaluative concerns: the questions of rigor and relevance}

Evaluative concerns for all research address the questions of rigor and relevance, which fundamentally attend to two closely related issues, those of quality and value. The issue of rigor or quality deliberates on the trustworthiness of the research process of a study and its findings; the issue of relevance or value evaluates how a study and its finding can be of use outside of its singular context. These are indeed two sides of the same coin, for without rigor, any claimed value is simply void, whereas rigor without relevance only turns the claimed rigor into a rigor mortis, dramatically speaking.

\footnotetext{
12 Interestingly for a supposedly 'collectivist' culture, the Thai use first name, rather than family name, as a form of formal address and identification. For informal situation, a 'nickname' is always used. Most, if not all, Thais have nicknames, normally given at birth, and the use of nicknames is a strong indication of intimacy, friendliness, or cordiality, rather similar to being on a 'first-name' basis in the Western culture. Addressing anybody in Thailand with his or her family name alone sounds extremely strange, unless it is done by a foreigner or in a Western-dominated, multicultural environment.
} 


\section{Evaluative concerns for qualitative research}

The issues of rigor and relevance are usually translated into the more commonly recognized terminologies from quantitative research tradition, namely the concepts of reliability, validity and generalizability. However, where qualitative research is concerned, the questions of rigor and relevance do not prompt such a straightforward solution (Cohen and Crabtree 2008, Emden and Sandelowski 1998, Spencer, Richie, Lewis, and Dillon 2003, Winter 2000). For quantitative research, the key criteria of reliability, validity and generalizability have attained a virtual consensus with regards to their exact definition and established significance, almost to a point of brandishing an incontestable 'status of a scientific holy trinity', as Kvale put it (1995: 19). It follows that there exists an abundance of sophisticated and powerful statistical procedures and techniques that are tested, tried and available for ready application by quantitative researchers who wish to ensure and legitimize the rigor and relevance of their work in all significant aspects.

Qualitative researchers, on the other hand, have as yet no such ready holy trinity at their disposal and must face with an array of on-going proposals on competing criteria and methods yet to be fully tried, tested, unified or integrated and confirmed even a status of 'likely' candidates for 'acceptable' evaluative mandates and instruments (Cohen and Crabtree 2008, Emden and Sandelowski 1998, Mays and Pope 2000, Sparkes 2001, Spencer, Richie, Lewis, and Dillon 2003, Tobin and Begley 2004, Winter 2000). In their critical and comprehensive reviews, Cohen and Crabtree $(2006,2008)$ catalogued at least thirteen major evaluative mandates, whereas Spencer, Richie, Lewis, and Dillon (2003) selected twenty-nine competing evaluative criteria proposals for detailed examination. Amid such contention and confusion, the dilemma an interpretive-qualitative researcher must face with is vivid and demanding.

This study is no exception, and I decided to adopt a strategy employed by many other similar studies, namely devising a 'recipe' of methods to ensure the study's legitimacy by integrating appropriate suggestions made in a collective wisdom from experienced scholars. Most specifically, I addressed the questions of rigor and relevance for this study under a principle widely advocated by prominent researchers - the principle that any evaluation of a study, with respect to both its rigor and relevance, makes sense only when done within the context of the paradigm underlying it (Ambert, Adler, Adler, and Detzner 1995, Cohen and Crabtree 2008, Creswell and Miller 2000, Davies and Dodd 2002, Healy and Perry 2000, Long and Johnson 2000, Maxwell 1992). The tendency to apply stringent evaluation criteria of validity, reliability and generalizability - exactly as defined for positivist or post-positivist studies - to research carried out under other paradigms of inquiry could end up in meaningless effort at best, complete misjudgement at worst.

Given the stated principle, I set out to compile a scheme of evaluative criteria from a critical survey of major available reviews, debates and proposals regarding evaluative criteria and procedures for qualitative research. The review resulted in a 'contingent' 
evaluative mandates and procedures devised for and adhered to in this study. ${ }^{13}$ Following is a brief account to explain this effort and its eventual evaluative schema.

Three major issues characterize the controversy on evaluative concerns for qualitative research: 1 ) what should comprise the exact criteria to ensure the desired attributes of quality and value in qualitative research? - or the questions of identification and definition; 2) how should these criteria be named? - or the issue of terminologies; and 3) what are appropriate methods or procedures that can be used to meet these criteria?. Competing standpoints abound in answering these three questions.

\section{Identification and definition of qualitative evaluation}

Debate on how best to define an appropriate set of criteria to ensure rigor and relevance in qualitative research revolves around one single matter: how far qualitative research could or should be judged in the same way as quantitative research. (Ambert, Adler, Adler, and Detzner 1995, Angen 2000, Golafshani 2003, Kvale 1995, Lincoln 1995, Lincoln and Guba 1985, Long and Johnson 2000, Maxwell 1992, Sandelowski 1986, Sandelowski 1993, Smith 1984, Smith and Deemer 2000, Stenbacka 2001). The various views on this concern range from a proposal to adopt the exact same criteria as those in quantitative tradition to a call for complete rejection of the notion of criteria altogether, with a wide variety of positions in between the two extremes.

From this range of perspectives, I adopted a view categorized by Sparkes (2001) as the 'parallel perspective' based on philosophical ground but with a practical flavour to it. The parallel perspective, in brief, takes a position that qualitative inquiry should be judged upon its own foothold, guided by its own nature that is distinct from that of quantitative research, but at the same time a set of qualitative criteria must in essence still be equivalent to its quantitative counterpart - hence the term parallel. Given the interpretive paradigm that governs this study and its qualitative approach, a straight application of an exact 'replica' of any positivist-quantitative standards and procedures for evaluation, however convenient and respectable that may be, would be awkward at best, meaningless at worst. To illustrate this assertion with an analogy, let us consider two similar yet distinct types of sport, that of speed skating and figure skating. Although the two genres share the same foundation - skating - they have entirely different nature and purposes. Hence, the two genres must adhere to two entirely different sets of criteria for judging a competition. Interpretive-qualitative research is

\footnotetext{
13 The effort involved a large body of such work (Altheide and Johnson 1994, Ambert, Adler, Adler, and Detzner 1995, Angen 2000, Chiovitti and Piran 2003, Cohen and Crabtree 2008, Creswell 1998, Creswell and Maietta 2002, Creswell and Miller 2000, Davies and Dodd 2002, Denzin and Lincoln 2005a, Emden and Sandelowski 1998, Emden and Sandelowski 1999, Fitzner 2007, Golafshani 2003, Hammersley 1987, Hammersley 1998, Hammersley 2007, Hammersley 2008, Healy and Perry 2000, Johnson 1997, Kvale 1995, Lincoln 1995, Lincoln and Guba 1985, Long and Johnson 2000, Malterud 2001, Maxwell 1992, Mays and Pope 1995, Mays and Pope 2000, Miles and Huberman 1994, Patton 1999, Sandelowski 1986, Sandelowski 1993, Seale and Silverman 1997, Smith 1984, Smith and Deemer 2000, Sparkes 2001, Spencer, Richie, Lewis, and Dillon 2003, Stake and Trumbull 1982, Steinke 2004, Stenbacka 2001, Tobin and Begley 2004, Tong, Sainsbury, and Craig 2007, Whittemore, Chase, and Mandle 2001, Winter 2000, Yardley 2000).
} 
analogous to figure skating, where the athletes are assessed based on a set of criteria that are qualitative in nature (even though eventually quantified in form). Just as it would be meaningless to judge figure skating based on speed skating criteria of precise numerical speed down to hundredth of a second, it would be equally futile to apply rigid statistical evaluation techniques to evaluate qualitative research.

Writing for 'scholars who do not engage in qualitative research and/or who are not familiar with its methods and epistemologies', Ambert, Adler, Adler, and Detzner (1995: 879) set out to explain the above philosophical standpoint in detail. Their arguments were echoed and embellished in several other works (Cohen and Crabtree 2008, Creswell and Miller 2000, Davies and Dodd 2002, Healy and Perry 2000, Long and Johnson 2000, Maxwell 1992). The argument from this body of literature inspires the following line of reasoning. Take the first desired quality in any research - accuracy, which addresses directly an inquirer's stance on truth value or what constitutes truth. As earlier expounded, interpretivist acknowledge multiple competing truths while positivists or post-positivist advocate one single universal truth. From this origin, the nature and purposes of research governed by the two paradigms depart in their analytical focus, mode of explanation, objective and finally the very definition of accuracy, as stylized in Diagram 2.7. In effect, because the key objective in positivist-quantitative research is to base its findings on hypothesized measures, precision related to such measures defines accuracy, leading to the applicability of myriad powerful statistical procedures to assess the accuracy of a quantitative study. By contrast, interpretivequalitative research emphasizes meanings, whose accuracy is enhanced not by precise reduction but rather by lavish elaboration, making depth and richness of research accounts a more appropriate definition of accuracy. For this reason, instead of statistical techniques, such methods as thick description and audit trail are more applicable to ensure accuracy in qualitative research.

Diagram 2.7: Comparative view of 'accuracy' as a desired attribute of good research

\begin{tabular}{r|c|c|}
\cline { 2 - 3 } truth: & $\begin{array}{c}\text { positivist/quantitative } \\
\sim \text { measures \& causal explanation }\end{array}$ & $\begin{array}{c}\text { interpretive/qualitative } \\
\sim \text { meanings \& understanding }\end{array}$ \\
\cline { 2 - 3 } analytical focus: & single universal truth & multiple competing truths \\
\cline { 2 - 3 } mode of explanation: & measures & meanings \\
\cline { 2 - 3 } objective: & causal & intentional \\
\cline { 2 - 3 } accuracy: & prediction & understanding \\
\cline { 2 - 3 } & precision & depth and richness \\
\hline
\end{tabular}

Note: Author's integration and conceptualization from selected literature (Ambert, Adler, Adler, and Detzner 1995, Cohen and Crabtree 2008, Creswell and Miller 2000, Davies and Dodd 2002, Healy and Perry 2000, Long and Johnson 2000, Maxwell 1992). 
The above treatment of accuracy elucidates the core principle and objective of a parallel approach: to devise a parallel schema of 'shared' desired attributes general to all research that comprises comparative interpretations of the same quality concept, one for positivist-quantitative and the other for interpretive-qualitative research. The schema devised for this study subscribes to this very principle.

\section{Terminologies: What's in a name?}

Next, a brief note on the choice of terminologies chosen for the devised criteria is needed primarily because this is a hotly debated issue. Here was where I decided to put a practical slant to the parallel perspective on the debate. Controversy regarding terminologies is usually not addressed in and of itself alone, but in conjunction with the discussion on identification and definition of criteria, making the issue even more convoluted. Not to belabour the subject, a simplified view on this debate is presented here only as an explanatory note to the position I finally adopted.

Broadly speaking, controversy on terminologies also spans a spectrum anchored by two extremes. Stenbacka (2001) well represents one extreme view in this debate which advocates a completely new set of terminologies for criteria applied to qualitative research. A classic example of such approach is the use of the term 'trustworthiness' in place of 'validity', as proposed by Lincoln and Guba (Lincoln 1995, Lincoln and Guba 1985). The argument for this stance is that, as Stenbenka argued, since '[o]ur vacabulary steers our minds and leads us to certain actions' (Stenbacka 2001: 551), there is a need for different terminologies to underscore the different evaluative concepts in qualitative research. At the other end, Long and Johnson (2000) reviewed quality concepts for qualitative research proposed to date and concluded that they amounted to virtually the exact same fundamental meanings of reliability and validity as defined in quantitative research, making coining new terminologies no more than giving a rose just another name. Metaphorically speaking, I resorted to not giving the rose any other name, yet qualifying each single one with a distinct scent and beauty of its own.

Perhaps the most scrutinized set of criteria for qualitative research to date is the one proposed by Lincoln and Guba (Lincoln 1995, Lincoln and Guba 1985), which, when examined closely, represents almost a mirror image of those established criteria for quantitative research, only with different names and one unique extra criterion (authenticity). Though widely recognized and discussed (Bryman and Bell 2003: 35, Spencer, Richie, Lewis, and Dillon 2003: 40, Tobin and Begley 2004: 391-392), these criteria have yet to earn a unanimous approval. The appeal of Lincoln and Guba's criteria set lies in their parsimonious parallel to well-known quantitative quality and value concepts. What makes the set slow to gain ready acceptance may have been because of the need to translate and get used to the new terminologies before one can match them to those already long established. This creates an unnecessary complication and invites a belaboured debate on lexicon rather than meanings. 
Hence, Long and Johnson's observation may hold merit in this regards, that is, 'nothing is to be gained from clouding the issue with alternative labels for what have been argued to be identical concepts' (Long and Johnson 2000: 31). 'What's in a name? That which we call a rose [b]y any other name would smell as sweet. ${ }^{14}$ Validity, reliability and generalizability by any other names would still refer to the well-regarded quality and value concepts, so to speak.

But then again, the concepts are not quite identical, as earlier expounded - the roses smell as sweet yet with different scents and aesthetic characteristics, so to speak. This inspired my practical twist to the issue of terminologies: taking the established terminologies and adding to them differentiating qualifications, which resulted in a parallel set of criteria for this study comprising five criteria that are easily comparable to those key well-known criteria in quantitative tradition. However, the familiar terminologies are explicitly qualified to denote their variant meanings in qualitative research. In effect, I propose an 'unidentical' twin of 'naturalistic' holy trinity of validity, reliability and generalizability to match the well-regarded statistical one. The reason for this tactic is to capitalize on the nature of human thought process: we understand new concepts best when we can easily relate them to concepts already ingrained in our mind. Thus, the use of familiar terminologies should serve at least two practical but important purposes. First, it would make communication and comparison across perspectives and paradigms more parsimonious and effective. Second, it would exploit the merit of an established set of criteria to enhance ready understanding and acceptance of its equivalent counterpart.

\section{Evaluative Criteria for qualitative research: a qualitative 'holy trinity'}

Having explained the rationale and position behind the criteria to evaluate qualitative research, this section now presents the eventual evaluative criteria devised and observed for this study.

Spencer, Richie, Lewis, and Dillon (2003) reported a comprehensive survey of published work on evaluative criteria for qualitative research and provided a thorough characterization and analysis of the major issues in this debate, painting a rather perplexing state of affair. Yet they concluded with an encouraging observation that eventually informed the development of the evaluative schema for this study. That is, despite the seemingly controversial nature of the debate, there actually appears to be a high degree of agreement concerning the two crucial questions on evaluation. The first question is, 'what make good research?' and the collective answer from the literature pins this down to five major quality, that is, accuracy, extendibility, topical relevance, applicability, and objectivity/neutrality. Of these, the first four are included in the devised schema as shown in Diagram 2.8. The criteria of objectivity/neutrality is excluded because of its incompatibility with the paradigm of inquiry that informed this

\footnotetext{
${ }^{14}$ Shakespeare's Romeo and Juliet (II, ii, 1-2).
} 
study. Instead, the issue of inquirer's viewpoint is addressed through the concept of reflexivity to be discussed shortly. Next, the second question in this debate then asks, 'what methods are effective to ensure such quality in qualitative research?', and an integrated compilation of widely accepted methods proposed in the literature is illustrated in Diagram 2.9 and will be duly discussed.

Diagram 2.8: Comparative evaluative criteria

\begin{tabular}{|c|c|c|c|}
\hline \multirow[b]{2}{*}{$\begin{array}{l}\text { Rigor and } \\
\text { relevance to } \\
\text { audiences ... }\end{array}$} & \multicolumn{3}{|c|}{ Evaluative criteria } \\
\hline & $\begin{array}{c}\text { fundamental \& } \\
\text { underlying all } \\
\sim \text { general/universal }\end{array}$ & $\begin{array}{c}\text { positivist/ } \\
\text { quantitative } \\
\sim \text { measures \& } \\
\text { causal explanation }\end{array}$ & $\begin{array}{l}\text { interpretive/ } \\
\text { qualitative } \\
\sim \text { meanings \& } \\
\text { understanding }\end{array}$ \\
\hline Research & $\begin{array}{l}\square \text { accuracy } \\
\square \text { extendibility }\end{array}$ & $\begin{array}{l}\square \text { construct validity } \\
\square \text { internal validity } \\
\square \text { statistical reliability }\end{array}$ & $\begin{array}{l}\square \text { analytical validity } \\
\square \text { interpretive validity } \\
\square \text { procedural reliability }\end{array}$ \\
\hline Practice & $\begin{array}{l}\square \text { topical relevance } \\
\square \text { applicability }\end{array}$ & $\begin{array}{l}\square \text { content validity } \\
\square \text { probabilistic gener- } \\
\text { alizability }\end{array}$ & $\begin{array}{l}\nabla \text { experiential validity } \\
\square \text { naturalistic gener- } \\
\text { alizability }\end{array}$ \\
\hline
\end{tabular}

Note: Author's integration from selected literature (Altheide and Johnson 1994, Ambert, Adler, Adler, and Detzner 1995, Angen 2000, Chiovitti and Piran 2003, Cohen and Crabtree 2008, Creswell 1998, Creswell and Maietta 2002, Creswell and Miller 2000, Davies and Dodd 2002, Denzin and Lincoln 2005a, Emden and Sandelowski 1998, Emden and Sandelowski 1999, Fitzner 2007, Golafshani 2003, Hammersley 1987, Hammersley 1998, Hammersley 2007, Hammersley 2008, Healy and Perry 2000, Johnson 1997, Kvale 1995, Lincoln 1995, Lincoln and Guba 1985, Long and Johnson 2000, Malterud 2001, Maxwell 1992, Mays and Pope 1995, Mays and Pope 2000, Miles and Huberman 1994, Patton 1999, Sandelowski 1986, Sandelowski 1993, Seale and Silverman 1997, Smith 1984, Smith and Deemer 2000, Sparkes 2001, Spencer, Richie, Lewis, and Dillon 2003, Stake and Trumbull 1982, Steinke 2004, Stenbacka 2001, Tobin and Begley 2004, Tong, Sainsbury, and Craig 2007, Whittemore, Chase, and Mandle 2001, Winter 2000, Yardley 2000).

First, two sets of 'audiences' are equally important for all research, those in the research and practice communities. In a nutshell, a study should contribute simultaneously to intellectual advance and practical use in order to be deemed to have respectable quality and good value. Thus, rigor and relevance must be simultaneously related to these two groups of audience. In general, this translates into two sets of desired attributes. From research perspective, two characteristics guarantee good research: accuracy and extendibility. To reiterate earlier explication on the different treatments of accuracy, for qualitative research, accuracy lies in the richness and depth of research findings and analysis. Extendibility for all research refers to the property that would make it possible for other researchers to extend the intellectual pursuit of a study, either by a straightforward replication, or more elaborated modification and extension. Whereas in quantitative research, this attribute is achieved 
through the stable precision of measuring and statistical instruments, extendibility in qualitative research is made possible through detailed and comprehensive accounts of the research process itself, via methods such as audit trail. The next two attributes address perspective of those in the practice community, namely topical relevance and applicability. First, a study investigates issues that capture social interest, or having topical relevance, and second, it delivers findings that can be applied to real-world situations, or offering potential applicability. As these two attributes relate to practical purposes, topical relevance and applicability carries virtually the same meanings for both quantitative and qualitative research: practitioners must find the study interesting and useful, either through numerical or analytical means, or both.

The four general attributes - accuracy, extendibility, topical relevance and applicability - are then translated into the holy trinity of validity, reliability and generalizability. When referred to in broad terms, these concepts can be argued to have the same general meanings for all research, quantitative or qualitative or mixed (Long and Johnson 2000). First, validity refers broadly to 'degree of approximation of reality' (Johnston and Pennypacker 1980: 190-191). For quantitative study, where approximation is by means of precise 'measuring' of a reality, validity then refers to 'the issue of whether an indicator (or set of indicators) that is devised to gauge a concept really measures that concept' (Bryman and Bell 2003: 77). For qualitative research, where the approximation focuses on gauging meanings of a reality, validity is more about 'represent[ing] accurately those features of the phenomena that [a study] is intended to describe, explain or theorise' (Hammersley 1992: 69).

Next, reliability refers generally to the issue of consistency of the entire research process such that a study is reproducible across various research settings (Bryman and Bell 2003: 74-75). For quantitative research, reliability is achieved through 'the capacity to yield the same measurement stability' (Johnston and Pennypacker 1980: 190191). For qualitative research, reliability 'refers to the degree of consistency with which instances are assigned to the same category by different observers or by the same observer on different occasions' (Hammersley 1992: 67) or to put simply, the degree of consistency in recording and observation. However defined, the ultimate aim of reliability is a high degree of reproducibility of a study across various research settings, with regards to time, place, inquirer, and respondent.

Last, generalizability - which is in fact a sub-set of validity, that is external validity refers to 'the degree to which research findings are applicable to other populations or samples' (Falk and Guenther 2007: 2). For quantitative research, probabilistic generalization techniques are the standard to ensure generalizability: as long as sampling methods for a quantitative study can be proven to carry strong probabilistic power, the study findings are believed to hold true also to the population which those samples 'truly' represent. For qualitative research, generalizability is another hotly-debated issue (Falk and Guenther 2007, Gobo 2008, Hellström 2008, Smaling 2003, Spencer, Richie, Lewis, and Dillon 2003, Stake and Trumbull 1982), the detail of which will be discussed shortly. Suffice for this early review to state that several interpretations on 
generalizability of qualitative research have been proposed, all of which hinge on the argument that probabilistic generalization is but one way to apply research findings to real-world phenomena. Alternative approaches to generalization have been proposed and substantiated to be applicable to qualitative research.

Thus is an overview of the parallel 'holy trinity' twins of evaluative criteria. As many have discussed, the exact meanings and types for each of the three concepts in the qualitative holy trinity have been debated at length in the qualitative research circle, with many competing definitions, typologies and categories. Spencer, Richie, Lewis, and Dillon (2003: chapter 5) provided a thorough review on the issues, with detailed examination of all prominent positions and proposals regarding the concepts of validity, reliability and generalizability. Their thorough review illuminated both bad and good news. The bad news is that there are unfortunately several competing proposals for the exact types of validity, reliability and generalizability that are appropriate for qualitative evaluation. The good news is that, despite the contention, most, if not all, proposals do not differ significantly in their underlying rationale, which makes it less excruciating when choices need to be made.

Certain choices were thus made for this study. Let us now examine how this study views the application of the holy trinity to qualitative research in particular. As outlined in Diagram 2.8, this holy trinity can be broken down into five key specific criteria for both positivist-quantitative and interpretive-qualitative research. The two sets are equivalent as well as different. The five evaluative criteria for qualitative research were derived primarily by translating key criteria for quantitative research to their equivalent counterparts for qualitative study. Then, the exact meaning of each qualitative criterion was defined based on selective integration of relevant discussions on that issue in the literature. This set of criteria comprises three types of validity, one of reliability and one of generalizability.

The first criterion for qualitative study is analytical validity, which is a counterpart of construct validity in quantitative research. Whereas construct validity has to do with the quality of the operationalization of concepts to be measured, analytical validity has to do with the categorization of concepts embedded in or emerging from evidence. This first criterion relates directly to the fundamental nature of qualitative research, that is, to deal primarily with capturing meanings from verbal and non-verbal evidence. Thus, one prime activity in qualitative inquiry is to decipher a pattern from an assorted array of verbal and non-verbal clues in order to form a conceptual representation of the distilled meanings. Coding and categorization are the tools qualitative inquirers employ to distil and define concepts and their meanings (Corbin and Strauss 1990, Creswell and Maietta 2002, Denzin and Lincoln 2003, Dey 1993, Dey 1999, Glaser and Strauss 1967, Miles and Huberman 1994, Rennie 1998, Silverman 2004, Strauss and Corbin 1990, Strauss and Corbin 1998). Specifically for interpretive grounded theory research such as this study, analytical validity is crucial during the coding and theorizing process, where grounded evidence must be analyzed to find emerging concepts and categories that will form the basis for its ultimate output: a grounded theory. A 
qualitative study of high analytical validity is one in which its findings present a credibly complete and meaningful categorization of relevant concepts for the theoretical theme under study. Because interpretivist paradigm advocates multiple views of reality, analytical validity as defined here includes an element of Lincoln and Guba's additional and distinct evaluative criteria of 'authenticity' (Lincoln 1995, Lincoln and Guba 1985). Thus, a high degree of analytical validity calls for an inclusion of as many views and concepts on a phenomenon as are discernable in the evidence.

Next is the second type of validity for qualitative research - interpretive validity, which is comparable to internal validity in quantitative study. These two counterpart types of validity share one commonality: they refer to the credibility in statements about the relationship between concepts under study. However, whereas internal validity deals specifically with causal relationship (e.g. $x$ causes $y$ ), interpretive validity deals more with intentional relationship (e.g. phenomenon $y$ is likely motivated by intention $x$ ). Interpretive validity as defined here is thus equivalent to 'validity of interpretation' suggested by Mason (2002: 191-192), a combination of both 'interpretive validity' and 'theoretical validity' proposed by Maxwell (1992), and a combination of both 'credibility' and 'conformability' in Lincoln and Guba's schema (Lincoln 1995, Lincoln and Guba 1985). For qualitative research, while analytical validity affirms that all relevant concepts are well identified and defined, interpretive validity assures that the subsequent conceptualized or theorized relationship among the concepts are credible and well grounded on observed and recorded evidences, with sufficient reflexive accounts vis-a-vis the inquirer's stance on the subject to qualify his or her interpretation.

The third qualitative criterion is procedural reliability, which corresponds to statistical reliability in quantitative study. The difference is defined here in a comparatively simple fashion signified by the qualifying adjectives. In quantitative study, several statistical techniques such as test-retest, split half, interrater, and so on, are available to assess measuring reliability and affirm that the instruments used could reproduce consistent results in other research settings (Fitzner 2007). For qualitative research, reproducibility and extendibility of research is ensured by detailed and transparent description of its entire process, including full description and disclosure of informant profiles, research platform or field work, evidence collection procedure, coding activities, analytical records, and inquirer's reflexivity. In brief, the research 'procedure' must be explained as completely and transparently as possible, such that similar studies can be carried out in other settings in a comparable manner. For qualitative research, it is not the measuring instrument and its results that must be reproducible, but rather the research procedure itself. In this sense, procedural reliability as defined here is similar to, though not exactly the same as, the concept of 'dependability' in Lincoln and Guba's schema (Lincoln 1995, Lincoln and Guba 1985). A slight difference in the emphasis on what can be reproduced marks the difference between procedural reliability and dependability. That is, in the evaluative schema for this study, the procedure more than the findings is what must be reliable. The rationale for this choice of emphasis is that findings in interpretive research by its very nature are a matter of 
interpretation, which can only be verified but not reproduced. Hence, reliability is geared towards the procedures and instruments (of which the inquirer is one) and thus more a matter of providing 'a clear account of how the research was conducted, [...] such that readers can see how the findings were derived' (Spencer, Richie, Lewis, and Dillon 2003: 65).

The fourth qualitative criterion for evaluation is the last type of qualitative validity in the devised schema - experiential validity. This is comparable to the concept of content validity in quantitative research. Content validity (of which face validity is a sub-set) refers to credibility of concepts and their hypothesized relationship based purely on mental reasoning involving no statistical analysis. In layman terms, it simply assesses whether the hypothesis as stated makes logical sense or not. Similarly, experiential validity refers to assessment of credibility of any claimed interpretation of a phenomenon based purely on experience. Simply stated, if a study, its narrative and interpretation resonate with readers' experience vis-à-vis previously encountered phenomena, that study is said to have experiential validity. In layman terms, it simply assesses whether the hypothesis as stated makes experiential sense or not. Hence, both content validity and experiential validity serve a useful first test of whether a study has potential to be rigorous and relevant, especially to the practice community. Put simply, if a practitioner with substantial real-world experience of a phenomenon under study finds the research claims to 'make sense' when he or she relates them to his or her experience, then that study exhibits experiential validity.

Finally, the fifth and last criterion for qualitative research is naturalistic generalizability. The debate on whether or not qualitative study lend findings that are generalizable to real-world phenomena can be dated as far back as Znaniecki (1934), whose concept of 'analytical induction' may have been one of the earlier attempts to relate a generalization possibility that is not based on statistical law (Gobo 2008). Gobo (2008) and Spencer, Richie, Lewis, and Dillon (2003) reviewed the range of such nonprobabilistic forms of generalization and implied one mutual conclusion that generalization can take a variety of manners, of which probabilistic generalization is but one, albeit the most known and celebrated. Probabilistic generalization has become the de facto standard of generalizability, with an unfortunate side effect of it being perceived as the only standard. However, qualitative researchers argue for other forms of generalization, including though not limited to, analytical/theoretical generalization (Mitchell 1983, Seale and Silverman 1997, Yin 2008), representational generalization (Lewis and Ritchie 2003), and naturalistic generalization (Stake 1978, Stake and Trumbull 1982). Lincoln and Guba, although seemingly advocating non-generalizability of qualitative research - 'The only generalization is: there is no generalization' (Lincoln and Guba 1985: 110-128) - still proposed an equivalent concept of transferability, which in essence resonates very much with many of the other forms of nonprobabilistic generalization cited above. That is, all these non-probabilistic forms of generalization rely not on statistical law of probabilistic sampling but on other manner of making inference, be it through analytical, representational, experiential, or trans- 
ferral processes, all of which could provide valuable inferential understanding of one phenomenon based on accounts of another similar phenomenon. Such forms of generalization are well-suited to findings in qualitative research.

To keep it simple, this study opted for Stake's concept of naturalistic generalization because it embraces many elements from other forms of non-probabilistic generalization while resonating well with the concept of experiential validity. Stake defined naturalistic generalization as follows,

Naturalistic generalizations are conclusions arrived at through personal engagement in life's affairs or by vicarious experience so well constructed that the person feels as if it happened to themselves.

(Stake 1995: 85)

Naturalistic generalizations develop within a person as a product of experience. They derive from the tacit knowledge of how things are, why they are, how people feel about them, and how these things are likely to be later or in other places with which this person is familiar. They seldom take the form of predictions but they lead regularly to expectations.

(Stake 1978: 6, emphasis added)

Notably, the concept of naturalistic generalization informed how I view case study research as a form of virtual reality creation, a subject already elaborated.

\section{Methods and procedures to ensure rigor and relevance}

Despite a vast number of proposals and intense debates on evaluative criteria for qualitative research, a surprisingly high degree of unanimity exists when it comes to the practical matter of what exact methods or procedures are available and effective to ensure rigor and relevance in qualitative study. Diagram 2.9 integrates these major methods and procedures in one detailed summary and catalogues how I employed each of them in this particular study. Because Diagram 2.9 provides summary details of what the methods and procedures are and their relevance to this study, only a few important observations regarding these methods and procedures need additional elaboration.

First and foremost, I must emphasize that methods and procedures to ensure rigor and relevance are not the same as evaluative criteria. This may sound verbose but an unclear distinction can actually create unnecessary confusion. From researchers' viewpoint, methods and procedures to ensure rigor and relevance are instruments used to help warrant the recognition and implementation of evaluative criteria. From readers' perspective, the use and report of such methods and procedures in a study helps in the assessment of how well it meets the evaluative criteria. As such it is wise to guard against any tendency to equate the use of evaluation methods with an automatic evidence of quality. More scrutiny on how these methods and procedures are employed must be exercised to arrive at a final verdict on a study's eventual rigor and relevance.

Second, there is no absolute one-to-one relationship between evaluative criteria and methods and procedures to ensure rigor and relevance. That is to say, each method or procedure may and can be an instrument to ensure more than one criterion, and vice versa. For instance, thick description assists in establishing all five criteria 
in varying manners, whereas the criteria of procedural reliability can be fulfilled through such methods and procedures as audit trail, thick description and reflexivity. As such, the more methods and procedures are used, the higher the likelihood that rigor and relevance are instilled in a study, which is in itself an exercise of triangulation.

Finally, I argue that the seven methods and procedures selected and described in Diagram 2.9 represent a distilled yet comprehensive stock of what have been proposed in the body of literature. Therefore they were all employed in the course of this study to ensure its rigor and relevance as explained in the diagram which I now present.

Diagram 2.9: Methods and procedures to ensure rigor and relevance in qualitative research

\section{1)Theoretical sampling}

A major type of purposeful sampling (a qualitative counterpart to probabilistic sampling in quantitative study), and a key method in grounded theory.

Theoretical sampling is expounded in Section 2.2 of this chapter.

Employed in this study as described in Section 2.2 of this chapter.

\section{2) Prolonged engagement and persistent observation}

\section{An investigating technique to ensure depth and richness in evidence collection.}

$[T]$ he purpose of prolonged engagement is to render the inquirer open to the multiple influences - the mutual shapers and contextual factors - that impinge upon the phenomenon being studied, the purpose of persistent observation is to identify those characteristics and elements in the situation that are most relevant to the problem or issue being pursued and focusing on them in detail. If prolonged engagement provides scope, persistent observation provides depth.

(Lincoln and Guba 1985: 304, emphases added)

Employed in this study in two manners: 1) extended field visits and intensive, open-ended interviews; and 2) a deep familiarity I have with the two cultures under study since I was born and raised as a Thai and have lived in the Dutch community for over a decade.

\section{3) Audit trail}

A procedure to ensure credibility and procedural reliability by amassing a collection of auditable materials.

The audit procedure for naturalistic inquiry was pioneered by Halpern (1983) and formalized by Lincoln and Guba (1985).

An audit trail is a detailed and transparent documentation and report of 'the whole procedure of data gathering and analysis [...], including raw data material, categorized data material, and the findings (Akkerman, Admiraal, Brekelmans, and Oost 2008: 262). 
A complete audit trail for this study is available as a collection that contains: 1) $e$ mail correspondences with informants; 2) audio tapes of interviews; 3) interviews transcripts; 4) field notes; 5) coding and theoretical memos; and 6) descriptions of research process throughout this manuscript.

\section{4) Thick description, with low inference descriptions}

\section{A technique to lend depth and richness in reporting of a study and its findings.}

Thick description was a concept pioneered by Ryle (1949) and developed into established ethnographic technique by Geertz (1993). It refers to a research account which 'describe[s] the setting, the participants, and the themes of a qualitative study in rich detail' (Creswell and Miller 2000: 128). It also implies the acknowledgement of multiple viewpoints or multiple voices in a narration.

'The purpose of a thick description is that it creates verisimilitude, statements that produce for the readers the feeling that they have experienced, or could experience, the events being described in a study. Thus, credibility is established through the lens of readers who read a narrative account and are transported into a setting or situation.

(Creswell and Miller 2000: 128-129)

Low inference descriptors refer to 'the use of description phrased very close to the participants' account and researchers' field notes [such as] [v]erbatims (i.e. direct quotations)' (Johnson 1997: 282).

Employed in this study in the delivery of the three thick descriptive cases (Chapter 3,5 , and 7) and the two vignettes (Chapter 8).

\section{5) Triangulation}

(including negative or deviant case analysis, and disconfirming evidence)

A technique to ensure richness and comprehensiveness of evidence, analysis and findings.

Triangulation concept was developed first with a focus on triangulation of methods and later expanded to other aspects of research, including data or evidence, inquirer, informant, and analysis or theorizing (Steinke 2004).

The term "triangulation" is taken from land surveying. Knowing a single landmark only locates you somewhere along a line in a direction from the landmark, whereas with two landmarks you can take bearings in two directions and locate yourself at their intersection. The notion of triangulating also works metaphorically to call to mind the world's strongest geometric shape-the triangle (e.g., the form used to construct geodesic domes á la Buckminster Fuller). The logic of triangulation is based on the premise that no single method ever adequately solves the problem of rival explanations. Because each method reveals different aspects of empirical reality, multiple methods of data collection and analysis provide more grist for the research mill.

(Patton 1999: 1192)

Triangulation is also described as,

The use of complementary methods, theories, data, or investigators in the research [...] to compensate for any one-sidedness or distortion that may result from an individual method, theory, database, or researcher.

(Steinke 2004: 185).

Disconfirming evidence is a technique that comprises 'search[ing] through the data for evidence that is consistent with or disconfirms' preliminary findings (Creswell and Miller 2000: 127). Negative or deviant case analysis is a similar technique focusing on ' $[1]$ ocating and ex- 
amining cases that discomfort the researchers' expectations and tentative explanation' (Johnson 1997: 282). These two techniques are virtually two types of triangulation.

This study employed all major types of triangulation (Creswell and Miller 2000, Johnson 1997, Patton 1999): 1) methods triangulation (in evidence collection, coding, and analysis; 2) triangulation of sources (multiple informants, and grounded evidence coupled with theoretical sampling of relevant literature; 3) analyst/inquirer triangulation (through expert supervisory support and peer debriefing); $4\lfloor$ theory/perspective trianqulation (through peer debriefing and review of literature); and 5) disconfirming evidence and deviant case analysis (through constant comparison principle and comparative case analysis).

\section{6) Reflexivity}

A technique to probe and report inquirer's viewpoints as a way to indicate how these are part of the constructed reality (Mauthner and Doucet 2003).

Reflexivity is the process whereby researchers report on personal beliefs, values, and biases that may shape their inquiry.

(Creswell and Miller 2000: 127)

Reflexivity starts by identifying preconceptions brought into the project by the researcher, representing previous personal and professional experiences, prestudy beliefs about how things are and what is to be investigated, motivation and qualifications for exploration of the field, and perspectives and theoretical foundations related to education and interests.

(Malterud 2001: 484)

Reflection boxes denotes reflexive accounts for this study, while use of variation in writing style provides further indication of where reflexivity was employed, particularly through the use of the first person commentary. I also made use of footnotes for a few reflexive notes, whenever suitable.

\section{7) Debriefing}

An umbrella term referring to a set of techniques that resort to having research accounts and its findings being critiqued and commented on by those outside of the investigating process.

Member check is one debriefing technique that 'consists of taking data and interpretations back to the participants in the study so that they can confirm the credibility of the information and narrative account' (Creswell and Miller 2000: 127). Strongly advocated by Lincoln and Guba (Lincoln and Guba 1985), this technique is still highly controversial (Angen 2000, Sandelowski 1993).

Peer debriefing is 'a process of exposing oneself to a disinterested peer in a manner paralleling an analytical sessions and for the purpose of exploring aspects of the inquiry that might otherwise remain only implicit within the inquirer's mind' (Lincoln and Guba 1985: 308) or a 'review of the data and research process by someone who is familiar with the research or the phenomenon being explored [... a] peer reviewer provides support, plays devil's advocate, challenges the researchers' assumptions, pushes the researchers to the next step methodologically, and asks hard questions about methods and interpretations' (Creswell and Miller 2000: 129). Compared to member check peer debriefing is a more widely advocated technique (Cohen and Crabtree 2006, Cohen and Crabtree 2008). 
Throughout the five year period that this study spanned, I presented provisional interpretation, findings, and theorizing results in over twenty sessions of formal seminars, conferences or workshops and informal gatherings. The peers in these presentations were either academic scholars or practitioners and their comments and critiques met the guidelines quoted above. Also, the dissertation promoter and supervisor acted throughout as the ultimate peer reviewer. This constitutes peer debriefing for this study.I decided to rule out member check due to its controversial nature and the potential high risk that this technique may be a doubleedged sword and lessen rather than enhance rigor, especially for research informed by interpretive paradigm, as argued by Angen (2000) and Sandeowskil (1993).

Note: Author's selective integration and application from selective literature (Akkerman, Admiraal, Brekelmans, and Oost 2008, Altheide and Johnson 1994, Angen 2000, Cohen and Crabtree 2006, Cohen and Crabtree 2008, Creswell and Miller 2000, Cutcliffe and McKenna 2004, Davies and Dodd 2002, Emden and Sandelowski 1999, Geertz 1993, Glaser and Strauss 1967, Halpern 1983, Healy and Perry 2000, Johnson 1997, Lincoln and Guba 1985, Malterud 2001, Mauthner and Doucet 2003, Mays and Pope 1995, Miller 1997, Patton 1999, Rodgers and Cowles 1993, Ryle 1949, Sandelowski 1993, Steinke 2004, Strauss and Corbin 1998, Tina 2004, Tong, Sainsbury, and Craig 2007, Yardley 2000).

H AVING THUS ELABORATED ON THE KEY METHODOLOGICAL THRUST OF THIS STUDY, LET US NOW ENter the virtual reality and experience cross-cultural intelligence in action with the first of the three thick descriptive cases narrated in the next chapter. 


\section{Chapter 3}

\section{CAse ADT}

\section{UnDutching the UnDutchables}

They tell me what they think I would like to hear. They tell me this is going well because they think I'd like to hear that it's going well. They are not lying but they are not telling me what is going on. So, in the end I can only go by the end result.

Khun Kees van Dijk

CEO, ADT

2006

\section{. 333<मse8.}

In the Netherlands, I wouldn't do it this way, no.

Khun Pim Kempster Managing Director, ADT Thailand 2006

\section{.3334>8E.}

The Dutch are very direct. They can really shatter things to pieces with their directness. 
THE very first interview I conducted for this study was with Khun Herman, a high-level executive of a Dutch medium size, international manufacturer of industrial products ( $\mathrm{Vi}$ gnette TMF). The interview was at the company home base in Waalwijk. The experience was pleasant and encouraging; I couldn't have started my study any way better than that.

Khun Herman was the second person who responded to my request for interview, but the first I scheduled an interview with to seize the opportunity before his upcoming trip to Thailand. He held one of the top positions at corporate level, but also was acting as the MD for the company's Thai subsidiary. I was quite nervous to meet with such a 'real' executive, but Khun Herman turned out to be a kind, interesting and thoughtful man. When we were e-mailing to arrange for my trip to the company $H Q$, he was genuinely concerned about how I was to get there and even helped me with the practical details of the trip.

I took a train and then a bus to Waalwijk and arrived there one hour earlier than scheduled, by intention. I knew enough to make sure I was on time for an important appointment with a Dutch. I was supposed to call Khun Herman once I arrived at the bus station. Having arrived one hour earlier, I just went 'wandelen' around the city centre until it was time. At the agreed-upon time, I called Khun Herman and he said he would come to pick me up. Ten minutes later, there he was, riding in an old but well-kept Mercedes. We chatted on the short ride to the corporate $H Q$.

Once we were there, I was surprised to see that Khun Herman had arranged an assortment of sandwiches for me. He was quite thoughtful to have imagined that I wouldn't have had time for lunch, given the appointment time. The interview commenced and concluded better than I expected. I was immensely impressed by the whole situation, especially by Khun Herman's unexpected thoughtfulness of my well-being. Meeting him was my very first formal encounter with a Dutch outside the academic world and it surprised me a great deal that he was not as 'Dutch' as I had expected.

Fast forward about one year later in Thailand. I was sitting listening to Khun Herman's 'prized employee' - the Regional Export Manager, Khun Ekachai, a Thai whose potential Khun Herman had expressed great appreciation. Khun Ekachai revered and respected Khun Herman but at one point in the interview he divulged a hidden resentment that Khun Herman could sometimes be too 'detached' from his employees. Khun Ekachai was quite hurt when Khun Herman didn't show any concern about his recent family trauma: something 'everyone around here expressed their condolences to me', according to him. I was puzzled. I know in my heart that Khun Herman was far from unkind. But as a Thai, I quite understand Khun Ekachai's story and his sentiment also.

How do I make sense of that?

How do YOU make sense of that?

How do WE make sense of that?

WELL, as it turned out, I had to start figuring that out, not by looking at the case of TMF, but rather at the story of ADT - my very first experience of 'making sense'. 


\begin{tabular}{|c|c|}
\hline Industry & data communication technologies \\
\hline Product/service portfolio & $\begin{array}{l}\text { design \& development, manufacturing, and sales \& } \\
\text { marketing of data communication equipments \& solu- } \\
\text { tions (modems, routers and other interface solutions) }\end{array}$ \\
\hline Market portfolio & $\begin{array}{l}\text { B-2-B (OEM, embedded and carriers), and consumer } \\
\text { (end-users) markets worldwide }\end{array}$ \\
\hline Core competency & $\begin{array}{l}\text { design \& development / data communication engineer- } \\
\text { ing }\end{array}$ \\
\hline Operating portfolio & $\begin{array}{l}\text { primary focus on design \& development; sales \& mar- } \\
\text { keting served to drive growth; manufacturing only } \\
\text { recently done in-house in the Thai division (prior to } \\
\text { this, production entirely outsourced) }\end{array}$ \\
\hline Relative size $^{1)}$ & small \\
\hline Headquarters & Spijkenisse, the Netherlands \\
\hline Divisional offices & $\begin{array}{l}\text { R\&D in the Netherlands, one manufacturing facility in } \\
\text { Thailand; sales offices in the Netherlands, Thailand, UK } \\
\text { and the USA; distributors and resellers in several coun- } \\
\text { tries worldwide }\end{array}$ \\
\hline Corporate culture & $\begin{array}{l}\text { key executives were all Dutch, greatly influencing the } \\
\text { company organizational and management culture; } \\
\text { young, fast-growing and dynamic in outlook, in line } \\
\text { with its industry; centralized but flat organizational } \\
\text { structure }\end{array}$ \\
\hline Workforce $^{2)}$ & $\begin{array}{l}\text { Worldwide: } 160 \\
\text { In Thailand: } 120 \\
\text { [1 expatriate MD; } 7 \text { Thai high-level technicians, 1-2 Thai } \\
\text { sales staff; } 100+\text { Thai shop floor \& lower level employ- } \\
\text { ees] }\end{array}$ \\
\hline Manner of entry into Thailand & accidental (part of an acquisition package) \\
\hline Operating portfolio in Thailand & $\begin{array}{l}\text { production, and sales \& marketing (for the Asian re- } \\
\text { gion) }\end{array}$ \\
\hline Years in business & 22 \\
\hline Years in Thailand & 9 \\
\hline
\end{tabular}

Note: 1) defined very loosely as small, medium, and large, based on rough benchmark vis-à-vis overall global operation of the entire corporation relative to average multinationals.

2) as of 2006 . 
P IM KEMPSTER, MD OF A THAI SUBSIDIARY OF ADT, A DUTCH MULTINATIONAL WITH BUSINESS in data communication technologies, had recently decided that he would spend the rest of his life in Thailand, after half a decade running the one subsidiary of ADT in Rayong, a seaside town east of Bangkok. Was he now a rare 'unDutched' among the many UnDutchables? He couldn't say. He only knew that, even though he was still as Dutch as he could imagine, he now felt 'at home' here in this place, thousands of kilometres away from his native land.

His friend and boss, Kees van Dijk, CEO of ADT, and a few other colleagues at the headquarters, started to say teasingly to Khun Pim: 'You are not Dutch anymore. You've become more Thai than the Thai'. A tease this might be, it was still a telling tease. On the one hand, it seemed natural that Khun Pim had to 'adapt' somehow to the new environment. He could not keep on as a fish out of water and still effectively command a factory full of Thai employees. On the other hand, how well could he keep on channelling corporate mandates if he had to adopt a mentality that was akin to his Thai staff but foreign to his Dutch colleagues?

Khun Kees, the CEO, appreciated the delicate situation but still felt that, in any event, corporate mandates must take precedence. At times, he admitted he was rather frustrated with Khun Pim's sloppy approach to the Thai. 'How and why has he lost his Dutch directness?' - Khun Kees often wondered about Khun Pim. Other colleagues also saw things differently. Khun Joeri, Corporate VP of Sales \& Marketing, a true Dutch, felt that he could only do his job effectively the best way he had always done it: direct and to the point. It might not be compatible with the Thai's way, but it was what worked for him in his function and he could not do his job any other way. Khun Maleewan, a Thai female engineer in charge of the shop floor people, performed her role as a living bridge and had to juggle daily between the two mentalities she was sandwiched in between.

Is this tangle web of myriad approaches, viewpoints, and sentiments a case of a glass half empty?

Or is the glass actually half full?

\subsection{Enter the UnDutchables}

ADT was a Dutch company founded in the early 1980's by five engineering graduates of the Technical University of Enschede. Initially ADT started with a focus solely on design \& development of data communication technologies. Until its entry into Thailand around 1997, the company outsourced all its production needs to third parties and concentrated mainly on its core competency: design and development of data communication technologies - both hardware and software, for a wide range of data communication equipments including end-user modems, high-end modems, routers, and data interface solutions for telecommunication carriers and other businesses. ADT's divisional office in Thailand was a fully-owned subsidiary of the Dutch ADT. 


\section{Corporate}

Until its expansion into Thailand, the company's main activities centred on R\&D, logistics, and sales \& marketing and its sole customer base was made up of only business clients, that is, OEMs (original equipment manufacturers), carrier companies, and other businesses that needed data communication solutions. After a period of expansion, involving merger and acquisition as well as organic growth, ADT was now operating a full cycle of R\&D, production, logistics, sales \& marketing, and after-sales services, serving both consumer and business customers worldwide. Its brands of data communication hardware and software solutions were well recognised in all the markets it served.

ADT was a classic case of high technology entrepreneurial success. A small group of young Dutch engineers founded ADT to explore and exploit their expertise in communication technologies for commercial purposes. Within just a little over two decades, the company had grown and branched out considerably, mostly in a 'seize the day' fashion more than through strictly planned manner. By way of acquisition, it had broadened its market profile (to serve both consumer and industrial markets) as well as its competence (taking up production, in addition to design \& development and sales \& marketing). However, ADT's core competency remained its R\&D innovative expertise in data communication technologies.

ADT operated in a fast-moving high-tech industry where R\&D capabilities as well as market responsiveness were the key to its success. ADT served the entire range of data communication technology market, with four major market segments: retail, OEM, embedded solutions, and carrier solutions. This meant that ADT must respond to a variety of customer needs, from retail products for end-users to industrial solutions for business clients.

As of 2006, ADT operated a full-range of activities: R\&D, production (in Thailand), sales \& marketing, and after-sales services and supports. Company headquarters was in Spijkenisse, the Netherlands while its R\&D activities were carried out at the original Enschede office. Production took place at the Thai plant in Rayong. The company sales \& marketing network spanned the entire globe with branch offices in USA, UK and Thailand, and a vast network of resellers and distributors worldwide. This extensive scope of operation was quite impressive, for a young and small company. ADT was set to expand itself further in the future.

The management of ADT remained very much in the hand of the original founders, the CEO and key-executives being among the co-founders. Management style remained essentially Dutch: no-nonsense, result-oriented, direct approach. Decision and organization structure was fairly centralised, with key decisions and activities managed virtually by the core key executives for the entire worldwide operation. At the same time, organizational structure appeared relatively flat, with a simple chain-ofcommand arrangement. This may have to do with the relatively small size and young age of the company as well as the Dutch egalitarian spirit. 


\section{Local Division in Thailand}

ADT's operation in Thailand came about as a by-product of an acquisition in 1997 of Company TWX, which owned a popular retail brand of end-user data communication equipments. The main rationale for the acquisition was to gain entry into retail market, where the acquired company had already developed a substantial base. At the time of the acquisition, Company TWX owned a 'skeleton' factory plant in Rayong, Thailand. The embryonic plant was considered initially to have no potential for ADT since it was barely functional and came with problematic profile.

Thus, originally ADT was not at all keen on developing and using the plant, particularly as its production so far had entirely been outsourced successfully to other manufacturers, both in Europe and Asia. However, incidentally, the Asian financial crisis that occurred at the same time as the acquisition made it suddenly very cost-effective to develop the plant as a result of the drastic devaluation of the Thai Baht. ADT decided to invest in the plant and experiment with having its own production. In any case, if this experiment did not work out well, the plant could still be sold without a loss given the low investment cost.

As it turned out, ADT found that one of the advantages of having its own production site in-house was that this would give the firm greater flexibility to carry out other activities which could not be done easily via outsourcing - activities such as experimenting with new concepts, producing small specialized series or prototype products, and so on. This became part of the motivation not to do away with the Thai plant but instead to expand it even further. By 2006, ADT's Thai production facility served as a platform to produce higher-end products that benefited from in-house supervision and yielded better margin. For lower margin, simpler products, ADT still made use of outsourcing to cheaper third party factories such as those in Taiwan and China. With its own manufacturing capability, ADT even started to take on production of high valueadded products for other parties beside that of its own brands.

As of 2006, ADT operation in Thailand was under the management of a Dutch MD who was transferred from the headquarters. The MD had a long history as part of ADT top executives and was considered someone who could provide the best 'channel' for transferring corporate mission and management directives from the headquarters to its Thai plant. The MD managed a team of about seven high-level technicians and engineers who were in charge of the factory operation that had about 100 local workforces.

At first, sales \& marketing activities in Thailand were carried out via contracted distributors. As business advanced further, ADT decided to develop its own sales \& marketing capability in Thailand, with a sales \& marketing office in the heart of Bangkok. In 2006, the corporate Vice President of Sales \& Marketing took charge of this operation. The first sales personnel recruited by the earlier 'short-term' MDs were not functioning in line with ADT's expectation and would be replaced with a new team recruited by the VP, Sales \& Marketing himself. Most likely, sales \& marketing team for ADT in Thailand would grow as the business expands further both within Thailand and the Asian region. 
However, as of 2007 ADT did not plan for a rapid growth and still preferred to keep its operation to a size that was compatible with its flat organizational style of management.

\section{The actors}

\section{CEO: Dhr. Kees van Dijk (Khun Kees)}

Dhr. Kees van Dijk was the top-most executive of ADT and one of the original founders of the company. Dhr. van Dijk was a Dutch in his fifties and had a long history with the company. As CEO he was in charge of its entire operation. His position was based at the headquarters but he kept a close eye on the company operation worldwide and travelled regularly to interact with its divisions. He paid regular visits to the Thai plant about twice or three times a year, to oversee the yearly results and also deal with formal financial matters. His main concern about the Thai plant was how to keep it 'managed' in a proper ADT way through the primary Dutch channel he had, the current MD of the Thai division and his long-time colleague.

Dhr. van Dijk is referred to as Khun Kees in the narrative.

\section{Reflection 5: Khun Kees - a Dutch who liked coriander roots!}

At first contact, Khun Kees came across as a stern, serious, high-level executive whose concern was only about the business. However, once I got to know him better, especially after meeting him the second time at a hotel lobby in Bangkok and seeing him with his people at the factory. I found him to be a quite cheerful and caring person. His concern about the Thai 'lax' management and business style seemed to contrast sharply with his appreciation of the other aspects of the Thai culture. For example, he took a class in Thai cooking and actually knew how to cook some of the most authentic Thai dishes and surprisingly appreciated the most exotic Thai ingredient (coriander roots). He was very appreciative of the Thai 'dignified' humility, as he put it to me. He was certainly appreciative of some Thai traits while annoyed with some others at the same time.

\section{VP, Sales \& Marketing: Dhr. Joeri van Emmen (Khun Joeri)}

Dhr. van Emmen was a Dutch in his thirties. An energetic, lively personality, he oversaw sales \& marketing operation for the entire company. He maintained regular contact with the Thai office because he was the core link between ADT customers and its three other key departments: design \& development, manufacturing, and after-sale support \& service. His primary concern was to translate well what the customers wanted to the rest of the company while maintaining professional and profitable relationship with the customers. One essential 'code of conduct' of ADT was that the Sales \& Marketing department must be the 'only' interface between the company and its clients. As such, Dhr. van Emmen played a crucial role at ADT.

Dhr. van Emmen directly managed the Thai Sales \& Marketing operation, which had an office in Bangkok, as well as co-ordinated very closely with the Thai production 
operation. One important metaphor at the ADT factory was that Dhr. van Emmen personified the factory's 'biggest customer' because he was the sole representation of ADT's entire client base. Dhr. van Emmen's main concern was thus how he could drive the R\&D, production, and support teams to deliver what the clients wanted in a manner that was best for ADT.

Because Sales \& Marketing in Thailand was only in its beginning phase, Dhr. van Emmen had yet to build up his local Thai marketing team. He had some concerns about how to recruit Thai personnel who would understand and carry out the company's policy well. At the same time, he also needed to constantly uphold the role of Sales \& Marketing as the only interface between ADT customers and its Thai production team.

Dhr. van Emmen is referred to as Khun Joeri in the narrative.

\section{Reflection 6: Khun Joeri - the axe itself!}

Khun Joeri certainly had the charm of an effective salesman. He was my first contact at ADT and helped put me at ease through his friendly but efficient manner. Interestingly, I learned later that Khun Joeri was in fact Khun Kees' son-in-law. With hindsight, I can see why Khun Joeri could be quite relaxed and direct towards Khun Kees, something I first ascribed only to the Dutch style of management.

Khun Joeri was nothing if not direct! A Thai staff described him humorously as 'the axe itself'. To me, he personified well the 'trading' nature of the Dutch: the WYSIWYG style (what you see is what you get). He didn't mince words and he gave the impression that what he said he would deliver 'would' be delivered, no matter what. To his clients, this must be very reassuring. But I can't help wondering if his starkly 'direct' manner of communication might be too strong a medicine to swallow for many Thais.

\section{MD, Thai Division: Dhr. Pim Kempster (Khun Pim)}

Dhr. Kempster was about the same age as the CEO and, as one of the founders of ADT, had been with the company since its conception. Prior to taking up the MD position in Thailand, Dhr. Kempster had been a crucial member of ADT's top management team, managing its $R \& D$ division. For that reason, it was believed that he would be best suited to help establish a sound management tradition for the Thai operation since he understood first-hand how ADT worked and what its fundamental missions and mandates were. Prior to coming to Thailand, Dhr. Kempster had had some exposure to the Asian culture from dealing with Asian producers and suppliers as head of the R\&D division he previously managed.

Dhr. Kempster managed the entire operation of the Thai plant and worked closely to provide linkage with the headquarters and other divisions of the company. His main concern was how he could manage the Thai plant so that it was in harmony with the entire organization while keeping his local Thai staff happy with how certain things must be done in a 'Dutch' fashion.

Dhr. Kempster is referred to as Khun Pim in the narrative. 
Reflection 7: Khun Pim - the unDutched Dutch?

Khun Pim came across to me as a very reserved, polite and kind executive. Once I got to meet him, even in a short meeting. I realized how he could be regarded by many Thai employees as an ideal boss. He was reserved, which was a desired characteristic in the Thai image of an authority - to designate proper distance in a non-arrogant way. At the same time, he showed sincere affinity for the Thai people in general, and his Thai employees in particular. This portrayed the image of benevolence - yet another desired trait of an authority in the mind of most Thais. Somehow, my impression of Khun Pim was that he was almost like an aristocrat Dutch, not a commercial one. I still have to fathom how I came to form this impression.

\section{Production manager/HRM: Khun Maleewan Manasakul (Khun Maleewan)}

Khun Maleewan was an important member of the small team of Thai high-level technical personnel who provided the daily supervision of the shop floor activities at ADT. An accomplished amateur athlete in her school years, Khun Maleewan was a strong woman in her forties who was unique in several ways. She was a self-made educated middle-class, having fought her way up from an underprivileged working-class background. A woman in a man's world of engineering, she had every reason to be proud of her success. She had extensive experience working for an impressive list of famous multinationals. She spoke fluent English, was highly capable in her functions, and well respected and demanded as a guest lecturer at several local educational institutes. She had qualifications and experiences in both engineering and human resource management, which enabled her to perform several key functions at ADT. Khun Maleewan left ADT for a couple of years to work for another company but decided to come back again because of the personal affinity she had for Khun Pim, the shop floor people, the work, and the company itself.

As Production Manager/HRM, Khun Maleewan's main responsibilities included supervision, training and management of the overall workforce at the ADT factory. Khun Maleewan saw herself as a representative of the Thai employees. Yet, her personality had several traits more common in a Dutch. She was a self-proclaimed 'Miss Blunt' and aware that she had been in conflict with many Thais because of her direct manner. For all practical purposes, she acted as one of the main linkages between the Dutch executive team and the Thai shop floor employees. She 'translated' Dutch (or rather Dunglish, if you may) into Thai, literally as well as figuratively, on a daily basis. Her main concern was to make sure that executive mandates got translated into shop floor action in a manner as accurate yet agreeable as possible. This required a balancing act of constantly concerting the wishes of two different groups of people, both of which Khun Maleewan herself belonged to simultaneously. 
Khun Maleewan appeared to be an interesting personality. She was very articulate and confident, in a quite pleasing manner. I found her to be perceptive and caring. She certainly didn't have a character of a stereotype meek and mild Thai lady. Yet, to me, she personified an intriguing image of still another stereotype Thai woman: one described in a well-known Thai saying as ' a woman with one hand cradling her child while the other hand wielding a deadly weapon to defend her people'.

I believe I was intrigued by Khun Maleewan because she shared a few similar personality traits and life experiences as mine. We were both strong women, from very poor working-class families, who had to constantly fight to prove our worth in communities that belong more to people of superior endowments and privileges. I wonder how this impression influenced my interpretation of her messages.

\section{Supporting cast}

Two other Dutch employees from the Netherlands that kept regular contact with the Thai subsidiary were the financial officer and the technical officer. These two dealt mostly with Khun Pim, the MD, and were rarely in contact with the Thai staff.

Together with Khun Maleewan, another five to six high-level technicians or engineers formed a small team of supervisory management. They all had higher education and good command of the English language. This team provided various supervisory functions and were the Thai personnel that had regular contact with the Dutch executives or their Dutch counterparts. The rest of the Thai staff of a hundred or so local shop floor workers rarely, if ever, had to come into direct contact with any of ADT Dutch employees. These shop floor employees did not have the command of English to communicate with the Dutch, nor did they need to do so in their functions.

\subsection{The accidental inheritance and its cultural riddles}

ADT plant stood out in the midst of a vast landscape that used to be simple farm land. The coastal town Rayong, and particularly the Banchang district where ADT plant situated, was rapidly being transformed from a rural agricultural province into a booming industrial estate. Rayong was originally a sea-faring and farming community where simple folks lived a humble but contented life. By 2006, however, the region fast resembled a common suburb of Bangkok by the day.

The location of ADT plant was relatively remote, though not really a disadvantage for an industrial plant. The facility looked immaculately modern, in stark contrast with its surrounding area of vast farm land spotted by a few old dilapidated local dwellings. The surrounding area would eventually develop into booming industrial estate, but for now, ADT had the first-mover advantage and could consider expansion comfortably. ADT had already bought some surrounding land area, part of which would be a site for a small housing complex planned for visiting personnel or clients from abroad. 
But one prominent house in this complex would be a future home for Khun Pim, the MD, who had decided that he and his family wanted to settle down in Thailand, at least for the foreseeable future. His children might be going back to the Netherlands for their education, but he and his wife would adopt Thailand as their home. This decision came about after only half a decade at ADT in Rayong. Was this a case of unDutching the UnDutchables?

Khun Pim's decision marked an unexpected turn of event, given that ADT hesitated at first to even hold on to the plant when it came with the acquisition that was motivated more by branding asset of the targeted company than by this poor physical skeleton of a factory in a remote place of a far-away land. Prior to the acquisition and the accidental inheritance, ADT had no interest in manufacturing its own products in the first place and only decided to hang on to the factory just because it promised to be a lucrative investment, one way or another. As it turned out, the investment looked to be a permanent one.

This here is a story of an accidental inheritance that may have indeed unDutched a few of the UnDutchables.

\section{An accidental inheritance}

The inherited plant seemed at first sight to be a feeble excuse of a factory, no more than four walls and a roof to boast of. Development of the factory and its facilities had been put in the hand of a couple of Taiwanese 'plant developers' - also part of the inheritance. After a few months of no advancement in developing the plant further, they were duly dismissed by ADT. The skeleton building was then left to be properly 'maintained' by a small group of Thai staff who were attached to the factory by virtue of a previous contract, until ADT knew what to do with it.

Just then, the aftermath of the Asian crisis that left the Thai Baht drastically devaluated suddenly reduced the cost (or value) of foreign investments in Thailand by almost a third. ADT's guilders were worth almost three times their value only a few months earlier. The plant could be developed into a fully-functioning modern manufacturing facility at a cost which was affordable, though still substantial. To sell it in the midst of the crisis would not bring in much. For ADT, it only made logical sense to invest and consider this a windfall gain rather than a futile burden. Yes, it meant threading into an uncharged area for the young engineering company that had never dealt with or ever wanted to handle the production side of their innovation. Yet, it presented a lucrative 'experiment'. If that uncharged area proved to be bountiful, it would represent a major step forward. If not, the plant could be sold at a price that would not be a loss anyway, given the unbelievably low investment and the likely future recovery of the Thai economy.

It was not a smooth riding to set up the plant. For one thing, ADT had to learn how to deal with the way things were done in Thailand, when it came to bureaucracy and the various local customs. As long as it was not illegal or unethical, this challenge posed no acute predicament. The Dutch executives even enjoyed some of the quaint 
rituals like inviting the local monks to bless the plant at the opening ceremony (with ADT making a proper donation to the local temple, of course) or having the local police as special guests at a luncheon party so they would feel that the plant was part of the community they were protecting.

Eventually the plant grew into a full-blown, modern, impressive production facility in need of proper management. ADT top management, led by Khun Kees the CEO, looked first into those 'local Dutch' expatriates, ones who had been working and living in Thailand for a long period. Surely, they would know best. They must have known the Thai well after all these years. And they were Dutch, toch! It should have been a perfect solution.

Unfortunately, the solution was far from perfect. Somehow 'they' were not really Dutch. At least, they were not able to deliver what should be delivered in a manner ADT would want. The factory continued to operate more in the 'relaxed' Thai style even under the 'local' Dutch management. For whatever reason, the original two 'local' Dutch MDs did not provide the needed link to channel corporate mandates to the Thai division. It was a bad experiment to put faith in their capability to provide the needed linkage. Even so, the factory itself turned out to be a good experiment that promised substantial potential. ADT was now certain that it would want to keep this 'Cinderella' inheritance and further expand and exploit its new competence in production. What it needed was just to manage the plant effectively, the ADT's way.

Khun Pim thus came all the way from the Netherlands to take up the helm of ADT Thailand as a messenger of corporate mandates. It was never the intention that Khun Pim would stay in Thailand for a very long period. His role was simply to establish an efficient operation there so that its management could be easily transferred to another capable hand later on. Khun Pim was practically part of the original ADT family. He would be a better channel to deliver corporate message. After all, 'the medium is the message', is it not?

In the beginning, Khun Pim was not permanently stationed in Thailand. He would be in Thailand for about two months then back at the headquarters for around two weeks. After some time, the alternating arrangement was abandoned and Khun Pim decided to move and stay in Thailand permanently, bringing his family with him. When the five year maximum period of expatriate status was up he decided to stay on without the expatriate status but as a permanent resident.

Thus, the accidental inheritance turned out to be a pleasant gift. Yet, with the gift of this Cinderella subsidiary also came a few cultural riddles that puzzled the minds of all involved. Let us take a close look at two of those puzzles.

\section{Deadline: dead or alive?}

Khun Pim was rather impressed with the Thai's willingness to serve and the different manner of daily living he met with as a top expatriate in Thailand. True, things could be quite inconvenient without the ready modern material advantages he was used to in the Netherlands. However, the inconvenience of rural Thai ways was easily made up by 
myriad helping hands in the figures of personal driver, cook, maid and even gardener, all pleasingly willing and very easy on the pocket. Yet, these helpers were far from cheap in the value they created. At first, Khun Pim was overwhelmed with the overflow of the willingness to please, suspecting that a monetary agenda must be behind all that. Yet, he later interpreted this to be the fabric of the Thai culture. He felt that here, people were there for each other. It was not constantly about 'getting and spending' and he appreciated deeply the non-material aspects of life the Thai's way had to offer.

What was also enticing to Khun Pim was the relaxing attitude to life and work he experienced in Thailand. He also developed a genuine affection for the Thai staff at the plants. They were willing to serve, humble in their manner, yet strangely dignified. The 'dignified' humility was also appreciated by Khun Kees, the CEO, who found it to be a refreshing change from the overt brown-nosing manner he observed in some other places. The Thai's willingness to please but not in an overly-fawning way struck the right cord with the Dutch, who rarely felt comfortable in any overtly non-egalitarian situation. Yet, at times, the readiness to serve, or rather the eagerness to please, could create a strange tension.

Both Khun Pim and Khun Kees knew very well that the general Thai staff were always 'trying their best' to please. For Khun Pim, this meant a reciprocal kindness on his part. He felt the responsibility for the staff who looked upon him to 'provide and protect'. Although he interacted mainly with the few top technicians and engineers, he was a daily figure at the factory. All knew him as 'our boss'. Khun Kees, by contrast, was 'the boss', one who operated somewhere very much at the top, to be revered yet also distant. As for Khun Joeri, the VP of Sales \& Marketing, he was the 'biggest' customer and so he also needed to be served, of course as a top client. Khun Joeri himself believed that he only needed to make his 'wishes' clear and then there should be no question about the delivery to fulfil those wishes.

Sandwiched in between Khun Pim, Khun Kees, and Khun Joeri on the one hand, and the hundred or so Thai personnel on the other, was Khun Maleewan. Khun Maleewan strived to 'serve' the wishes of her Dutch bosses and to 'please' the expectation of her Thai staff, as much as it was reasonable and practical. It's a daily balancing act of translating a lot more than the literal English language into Thai. Oftentimes, she felt as if she was just a good old car bumper, absorbing the shock and preventing severe damages to all parties. She considered herself a very direct, down-to-earth person, yet she realized the Dutch could be even blunter than she could ever be. Still, Khun Pim, despite being a Dutch, seemed to be softer than she was. Thus, in this midst of diverse approaches and styles, she had to operate and provide the needed linkage and cushion. She knew that for the Thais, total directness can be petrifying and so she tried to act as a shock absorber for her Thai people. At the same time, a no-nonsense person herself, she often had to step in to deliver the bad news instead of letting Khun Pim do that. First of all, Khun Pim could not be so direct anyway. But most importantly, Khun Pim stood for the company to the staff; thus it would be a better tactic to keep 
up the image of Khun Pim as a benevolent boss, something that was important to any Thai workforce. Thus, a lot of times, while Khun Pim played the good cop, Khun Maleewan took on the bad cop's role.

Take the routine case of setting up 'delivery schedule,' for example. As a Production Manager/HRM, Khun Maleewan was directly responsible for scheduling the resources, physical as well as human, so that production could be done to meet delivery requirements, with regards to quantity, quality, and time. How should one set up 'delivery schedule' that would serve and please all parties involved?

ADT served a very demanding client-base and competed in a computerised technology market which changed so rapidly that stockpiling was not a viable solution. Put simply, the clients wanted the right products, at the right time, with the right quantity and quality. What this meant to Khun Joeri was that once a commitment was made for a delivery, it had to be delivered as and when committed. Delivery schedule for Khun Joeri was in effect a deadline with the emphasis on dead. Khun Maleewan knew this of course and always tried to set production schedule so that it matched with Khun Joeri's expectation. For the factory, however, that commitment could be no more than a delivery schedule - as in 'intended' date of completion, or a point of reference. Khun Pim on his part, could only trust that Khun Maleewan and her team would try their very best to keep to that point of reference.

Unfortunately, often enough, things did not go as 'scheduled' and the delivery dates had to be 'rescheduled' - sometimes more than once. At the factory, this was a matter of working harder to meet the new point of reference. But for Khun Joeri this could spell disaster. He needed to deal with anxious or, more often than not, disgruntling clients. He might even need to take costly action to keep the 'deadline' alive, such as having the shipment delivered by expensive air-cargo instead of cheaper sea freight. This dented the profit margin and did not reflect well on Sales \& Marketing. As it was, Khun Joeri and Khun Kees faced pressure from customers everyday to deliver on schedule. How could this pressure be felt just as acutely at the Thai factory, just so delivery schedule would translate into delivery deadline to them?

Khun Pim had experienced that kind of pressure before and certainly knew how it felt. Yet, here and now he also witnessed how 'hard' his staff tried to serve and realized that putting pressure was not really the Thai way of getting results. And his staffs were certainly Thai. At the same time, Khun Maleewan also felt the pressure. She knew when Khun Joeri - 'the axe' - said he wanted things delivered, it should be delivered and she tried her best to keep to the estimates. Still, she could only push the factory so far. The production was these people's jobs, yes, but it was not their lives. She wished the schedule could always be a tad more realistic as a reference point and a bit less holy as a deadline.

How could everyone be served and pleased at the same time? How could the delivery deadline and delivery schedule be synchronized? 


\section{Three strikes and you're out}

Murphy's Law governed the ADT plant every so often. That was to be expected. Nobody at ADT had the unrealistic expectation that mistakes would not be made. Still, mistakes could bring on complications that were beyond its simple direct consequences. The question was: how should one deal with mistakes?

ADT had a strict policy that only Sales \& Marketing Department was the only focal point for all communications with customers. This code of conduct enabled ADT to keep its tactical and strategic responses to market as solidified and consistent as possible. Khun Joeri did not really mind dealing with customers' comments, concerns or even complaints regarding ADT products. It did not matter where in the company the concerns were related to, be it design \& development or production. To deal with customers' requests and complaints was after all part of Khun Joeri's job. The information he got from such dealing could be used to improve future operation. In some cases, He could even use such cases to leverage his negotiation with the clients. If a customer had any issue with ADT's products or services, Khun Joeri and his team were there to deal with it. All in a day's work.

But the clients sometimes did not really care to observe ADT internal code of conduct, like in the case of a Singaporean client who took it to himself to approach the factory directly regarding dissatisfaction with a particular shipment. To the client, this was the shortest route to having his concern addressed. The Thai staffs at the factory who received the complaint were very embarrassed and anxious. They knew that they should tell the client to go to Khun Joeri but they suspected that the client was reluctant to do so for a combination of reasons. The client operated in Asia and it seemed more convenient to contact the Thai factory than the Dutch office. All that the client wanted was a direct, instant response he could get with a face-to-face meeting or realtime telephone conversation, without having to bother with time-zone difference or delayed e-mail communication. Moreover, most local clients preferred to deal with their local counterparts. To most Asians, it could be quite uncomfortable to approach and deal with the Dutch who were too far away and often too direct for their liking.

The Thai personnel concluded that to direct the customer to Khun Joeri was too awkward and irresponsible a solution. To the Thai employees, it was their obligation to please the client and rectify the shameful situation that arose out of their domain. They did not feel comfortable even to refer the problem to Khun Pim, the MD. In the end they tried their best to satisfy the client's request exactly as asked. After all, such direct dealings were not uncommon in most Thai business operations. Why involved Sales \& Marketing when it was actually the factory's fault? All's well that ends well.

Until Khun Joeri found out what had happened. This made his job incredibly difficult. He now had to try to sort out the double problems of rectifying the client's original concern, as well as restoring the undesirable consequences of this criss-crossing of communication lines. The factory was thus given a clear warning - a written announcement from the top boss, Khun Kees the CEO, who was not amused with the incident either. 'Please next time, if and when the factory get such a request from a 
customer, whoever that is, refer the customer to Sales \& Marketing and do nothing else whatsoever until you hear from your biggest customer - Sales \& Marketing Department, or Khun Joeri..' End of Act One.

Act Two: a similar incident came to pass. This followed again by a similar exchange between a client and the factory. It ended up again with the same 'deal' to rectify the 'mistake'. Everyone should be well-served and pleased. Alas, Khun Joeri and Khun Kees were certainly not. Another, this time very stern, warning was issued and made clear to all parties. It was specifically stated that the next time such non-observant of the code of conduct for a proper line of communication occurred, somebody would have to take the blunt of the consequences. Three strikes and you're out. End of Act Two.

Khun Pim the MD was not happy for Act Three to ever come to pass. He knew it was a common practice back in the Netherlands for such a reprimand to be issued and take effect. Yet he felt ill at ease seeing that treatment applied to the Thai people - $h$ is people - at the Rayong plant. They did their best with the best intention and might not take the reprimand as it was intended by the headquarters. Khun Joeri of Sales \& Marketing cared more that such misconduct would not occur again to interfere with his job; in his view, the Thai just had to learn to live with company's policy - simple as that. Khun Kees the CEO knew of course that the mistake was a result of a good intention, which made it even more difficult for him, because that good intention did not resonate with the best practice, particularly in this case. Something needed to be done to ensure that the best intention would also be compatible with the best practice.

Khun Maleewan agreed with Khun Kees in this respect but she also knew her people and the clients enough to realize that a 'stiff rod' approach would do no good. She settled for a balanced act. She would let Khun Pim do what he did best: being a benevolent boss, the good cop, while she herself took on the role of a disciplinary engine, the bad cop. This had worked well so far. The people needed to have a kind boss to put their faith in but they also needed to be injected some disciplines at the same time. As Khun Maleewan saw it, bad news to Thai employees had softer blow coming from a fellow petite, female Thai supervisor than from the top Dutch bosses. And so be it - a bumper she would be.

The intriguing questions remained. How could the best intention result in such unintended tension? How could the best intention be aligned with the best practice?

\section{The bottom line}

Yet, this was not really a completely tragic tale. These days, ADT Thai factory was operating and contributing to the corporate advances. Khun Pim was happy to continue his function as the MD for the Thai subsidiary, most likely until his retirement. Khun Maleewan, who left ADT to work for another company for a period, was now happy to be back at the plant she had helped to grow, working with the bosses and the staff she had developed personal affinity with. Khun Joeri was busy establishing his Sales \& Marketing team as well as keeping close contact with the factory. 
And Khun Kees, despite periodic concerns over the Thai plant, did not consider it to be unmanageable. As Khun Kees summed it up, 'Let's put it simple. If I can get the products in at the right time, with the right price, and of the right quality, whatever happens there, it doesn't matter. So far it's getting that way.' Khun Kees believed that after almost a decade, ADT had gradually learned the rope to strike an acceptable balance of approaches that would make future operation of the Thai plant smoother and less prone to cultural glitches. Not that he expected it to be smooth riding from now on, but such was life and everybody would have to take it as it came.

The accidental inheritance had lead ADT into an uncharged area in more ways than one. And it looked like the 'uncharged' area was steadily turning into a familiar ground. All in good time.

\subsection{Deciphering the cultural riddles}

The story of ADT highlights certain properties that characterize the majority of crosscultural encounters. Two such properties of any cross-cultural condition are most distinct in the above narrative: first, a multitude of competing meanings, and second, a certain degree of 'unDutching'. The following interpretation elaborates on these two properties of cross-cultural condition with a focus on the interfaces between the two national cultures: Dutch and Thai. Yet, as the analysis unfolds, other levels of cultural interfaces, beside that of 'national' level, also emerge to reveal the underlying intricate cultural webs.

\section{A multitude of competing meanings}

What was most striking in both incidents was that each character had his or her specific meaning of an act or issue he or she was facing with, even though all characters were involving in the exact same incident. A multitude of competing meanings created tension, especially when characters were not aware of the existence of other meanings. Tension could also arise even when actors were aware of the competing meanings but did not quite comprehend or appreciate what those meanings signify.

The following interpretation highlights a few major concepts to demonstrate what possible competing meanings transpired in the narrative of ADT.

\section{Time and commitment}

An obvious issue underpinning the issue of 'delivery schedule' v. 'delivery deadline' is that of time and commitment. For the Sales \& Marketing department, particularly Khun Joeri, delivery appointment denoted a guarantee of commitment. It designated what he guaranteed to delivery to customers, which equated to what the production line guaranteed to deliver to him. It was a deadline, meaning failure to fulfil this commitment would result in a fatal consequence. At the most apparent level, Khun Joeri's notion of time and commitment revealed his Dutch trait. For the Dutch, time is a valuable resource and appointment is a commitment (King 1993, Lawrence 1991, Vossestein 2004). In the Thai culture, by contrast, time is usually seen as something one 
should take command of and not be subject to (Cooper 2004, Holmes, Poonprasit, and Komin 2006, Holmes and Tangtongtavy 1997, Klausner 1993). A popular saying in Thailand asserts: 'Take it slowly and you'll get doubly rewarded'15. As such, Thais tend to take appointment not that seriously but merely as a point of reference, something that you set up so you have an idea of your target. Not meeting an appointment means no more than that either the target is wrongly set or something else has happened that has not been included in the estimation. In such a situation, for the Thai, a new target can be set and life goes on, no fatality needed.

Obviously, this interpretation draws on only one level of the meaning of time and commitment: the level influenced by national culture. Yet, in the incident on delivery schedule v. delivery deadline, each character's meaning of this issue was actually a blend of meanings which was only partly influenced by national culture and partly influenced by other cultures that a character belonged to. Khun Joeri, for example, took time and commitment extra seriously because of his profession and function as a Vice President of Sales \& Marketing. Khun Maleewan, on her part, had extensive experience in modern-day production line and was used to working under the pressure of deadlines. But at the same time, she was supervising the Thai workforce, who she knew full well would not take to deadline that seriously. So, to her, delivery schedule was a coin that had two sides, a sort of 'best estimate of commitment' if you may. This notion reveals a blend of several cultural forces - national, professional, functional, and departmental, to list the most obvious - that simultaneously influenced how Khun Maleewan looked at time and commitment in this particular incident.

\section{Motivation: pressure v. persuasion}

Both incidents in the narrative created a visible tension, which each character felt and sought to resolve, in his or her own way. To the Dutch executives, particularly Khun Kees the CEO, the most efficient solution was to put pressure on the workforce to meet the deadline or to observe the company's codes of conduct. From a Dutch viewpoint, this was the most efficient way to motivate people: that is, by putting a direct demand and spelling out the consequences of any failure to meet that demand. In addition, Khun Kees, as a business executive in a fast moving industry, operated constantly under pressure from all sides, which functioned as the key motivation for him to operate effectively and compete successfully. Putting pressure on people at the Dutch office had always worked for him. Naturally, Khun Kees considered it his duty to put pressure on Khun Pim, so that Khun Pim would pass the pressure on to the workforce.

Khun Pim, in comparison, having witnessed firsthand how the Thai carried on their lives, realized that pressure was not something that would motivate the Thai people. On the contrary, direct, stern pressure accompanied by harsh warning of a conse-

\footnotetext{
${ }^{15}$ This is a non-literal translation of 'cha cha dai pra song lem ngam', whose literal translation is 'Slowly, slowly and you will get two beautiful swords'.
} 
quence of failure could often be seen as a threat, and work to demotivate rather than inspire most Thais.

Khun Maleewan, as an atypical Thai who preferred direct approach to getting things done, appreciated the value of pressure. Her strong character could have been influenced by a cultural value of a special social class she belonged to, namely the selfmade professional. Yet, she also appreciated the Thai style of motivation that favours gentle persuasion, as opposed to direct pressure. Every educated Thai of her generation was taught the folk lore of Koe Nantawisan, a tale that celebrated the virtue of genteel kindness in an authority figure ${ }^{16}$. The imprint of this kind of teaching in the mind of the Thai is very vivid. Most Thais tend to sub-consciously absorb it message as a guideline of behaviour. When they are in a subordinate status, they prefer kind, gentle bosses to forceful, stringent ones. When they are in a superior position, they strive to show benevolence (Atmiyanandana and Lawler 2004, Chatterjee and Pearson 2002, Holmes, Poonprasit, and Komin 2006, Holmes and Tangtongtavy 1997, Komin 1989, Komin 1991, Lawler and Atmiyanandana 2004, Lawler, Siengthai, and Atmiyanandana 1998, Siengthai and Bechter 2004, Siengthai and Bechter 2005).

Thus, Khun Maleewan was operating under an intricate image of a boss that was a blend of many cultural meanings: the Dutch and Thai cultures, as well as her other cultural influences. Her solution resembled a 'good cop, bad cop' strategy. She would take on the role of the bad cop and let Khun Pim play the good cop's role. Her rationale was two-fold. First, as the top Dutch boss at the Thai plant, Khun Pim personified ADT, so it would be in the company advantage for the employees to have a good impression of the one boss who was a father figure for ADT. Secondly, given the Thai's abhorrence to any 'loosing face' kind of situation, a tough word from a foreign male boss carried a double shame: a shame of an inferior and a shame of being a misbehaved Thai. A harsh reprimand from a female Thai boss could considerably soften the blow of the double shame by more than half, by eliminating the complex embarrassment of being 'judged' badly by an 'outsider' who was also somehow a father figure. To Khun Maleewan, this strategy combined the best of both worlds, although it was certainly a lot more complicated to carry out.

This may look like a simple carrot v. stick issue of motivation. Yet, it is not that simple. The Dutch view's of pressure is not exactly a stick and the Thai view of persuasion is more than just offering a reward. Added to that is how this two distinct national views on the same issue can be further interpreted within an individual under the forces of other cultural influences. In the above analysis, for example, the influence of gender as well as work status was apparent. Had Khun Maleewan been a typical male engineer, the solution taken could and likely would have taken a different shape.

\footnotetext{
${ }^{16}$ This folklore tells a story of a cow who did not move one bit to work the field when hearing a rude shouting command from his farmer boss, but was later inspired to work extremely hard after his boss realized the negativity of his old style and changed from rude command to genteel, polite request for help and assistance. The cow, or Koe Nantawisan, symbolizes the soul and humanity of the subordinate.
} 


\section{Mistake: shame or guilt?}

In the second incident, a multitude of competing meanings of 'mistake' played a subtle role. There were at least two layers in deciphering the meanings of mistake: what mistake signifies and what is identified as a mistake.

Thais tend to look at mistake with apprehension. This relates substantially to the abhorrence of 'losing face' (Holmes and Tangtongtavy 1997, Komin 1991, Komin 1989, Siengthai and Bechter 2004).To most Thais, it is very important to be perceived well by others in the community. And to be perceived well is achieved mostly by maintaining a respectable, flawless appearance, reinforced by the eagerness to 'please' others. In reverse, anything that signifies a weakness, failure or inferiority is to be ignored, avoided, covered up, or instantly rectified. Mistake signifies negative quality and is a cause for shame. While Thais are shameful of mistake, the Dutch tend to take it simply as a sign of inefficiency or incorrect choice (King 1993, Lawrence 1991, Vossestein 2004). To most Dutch, one learns from one mistake and moves on. To learn from a mistake, one first needs to acknowledge it, or have it pointed out in the face. As such, to repeat the same mistake in the Dutch eyes is a sign of either inexcusable ignorance or misguided stubbornness.

The above competing views of mistake explain partly why the Thai employees acted the way they did when confronted with a client's complaint, and why the Dutch were not amused at the repeated violation of an important code of conduct. They also explain why there was a mismatch of identification of what exactly was 'the' mistake. The Thai staffs felt shameful of and responsible for having delivered 'bad' products and at the same time when confronted by the client, identified themselves as part of the company. They must have felt doubly shameful: at having produced bad products and embarrassed on behalf of the company. While the Thai looked externally, the Dutch were more concerned with the internal implication of the mistake. Khun Kees and Khun Joeri were much less concerned with the defect in shipment than with the outright violation of the company's key code of conduct. To them as executives, the consequences of the production mistake were at operational level, while violation of code of conduct struck at company's strategic and tactical cord. The incident of mistake and its consequences thus illustrated a rather tangled web of mismatched meanings of mistake.

\section{UnDutching as a prerequisite for cross-cultural encounter}

The multitude of competing meanings forming intricate cultural webs that characterize cross cultural encounters leads to a requisite of some 'unDutching'. First, every meaning that transpired in the case was simultaneously influenced by a multitude of cultures that make up a character. Each of the characters belonged to many cultural groups simultaneously - national, regional, organizational, ethnic, professional, social class, gender, and many others. As the case illuminates, how each character formed a meaning for an incident or issue was a blend of all these influential cultural forces. To add to the complexity is the 'dynamic' nature of this blend of cultures. It is dynamic in 
many levels. First, all cultural forces were in constant flux and so were their influences. Then, each character was shaping and reshaping his or her cultural profile on a daily basis based on his or her continuing experience in the world. More, each character was constantly shifting the relative 'weight' of the cultural influences according to a particular context.

Yet, the competing meanings and the fluctuating and complex nature of such diversity is simply a fact of life. The glass is neither half-full nor half-empty; it is simply filled. Whether one would think of the glass as half empty or half full depends on what the content of the glass is and how one interpret it in relation to the context. This is thus highly relative and contextual. Still, from the narrative, we can trace a few hints of how one can inspect the content of the glass and make the best out of it. Followings are a couple of 'rules of thumb' that emerge out of this case, which together signify that a degree of unDutching is always called for in a cross-cultural encounter.

\section{Let's call the whole thing off}

Maintaining positive energy in order not to be tangled up in the intricate cultural webs were elements that lurked behind the scene in this narrative. It appeared that once the characters got over the existence of the multitude of competing meanings and the desire to dogmatically strive for one single meaning, they turned their energy into dealing with the actual situation instead. A level of frustration was always present at first, but then those who faced the fact usually turned to find a way to work with, or around, the situation. Some even turned it into an advantage.

To accept competing meanings as a fact of cross-cultural life appeared to be the first step to operate in a cross-cultural condition.

\section{Something's got to give}

In the midst of the forces of multiple competing meanings, something has got to give. This means simply that adjustments are always called for in order to deal with the tension that are always present in cross-cultural life and turn that tenstion into a positive force - a case of some 'unDutching,' if you may.

Adjustments can come in many ways, shapes and forms. A character can adjust his or her attitude or style. Or, by contrast, a character can attempt to adjust the attitudes or styles of other actors. Alternatively, context can be shaped to ease the tension or routines can be revised or invented to accommodate the multiple meanings. The key to the unDutching is not a prescription of what, how, where or when to adjust but a constant awareness and seeking out for necessity and opportunity for adjustment. As far as the case goes, specific instructions for adjustment cannot be detected because the actual appropriate adjustments were relative to the context and governed more by heuristic than formulaic routine. 


\subsection{Final words}

\section{Khun Kees}

I personally believe that the relationship must be good but it's the result that counts. If the good relationship helps to get good results then that's good but it's not always the case. It could sometimes be a bad thing to have a good relationship. Every company is set up to make a profit, so it's the result that must take priority, not the relationship. The relationship must not be above the result. You do not build a company to have a good relationship with the people who work for you. That's not what a company's for.

\section{Khun Joeri}

But I do not think I'll 'adapt' to it. Sometimes I may upset people but to be frank, at the end of the day, it's OK. We cannot always be nice. Sometimes we need to get things done and we need to be a little bit more to the point. ... It's not my intention to insult people, but I need to get things done. Even my boss sometimes say I could be a little bit more, what do you say 'political'. I've been told often that you need to be 'political' and soft with the Thai people. But I think I can deal with them my way. Even with the customers in Thailand. I'll go to them and actually get to the point. I mean yes you can do it in a nice and friendly way and be political but it'll take forever.

\section{Khun Pim}

If Dutch people come here to Asia, they need to understand that Asian people, they don't like to say something that is not nice for us. So, they'll always try to bring it up in a positive way. They'll ALWAYS try to do that. If you ask somebody something, the answer is always 'yes' but the yes is not always a yes. I learned that from experience. I first always ask in a direct way and then if I get yes, I'll look to see a little bit from the attitude of the people if it's really yes. If not, then I'll ask again in a different way and get another answer. Then I'll even ask again the third time in another different way to get another answer. This way, I can collect the information and then I can make a decision, ok, this is really the answer. Then I try to anticipate on it and then I'll ask them if this is the answer. Then SUDDENLY they'd start talking. In the Netherlands I wouldn't do it this way, no.

\section{Khun Maleewan}

I think you first have to look at the actual personality and character of the particular individuals you are dealing with. Then you have to look at who they are, what role and status they have in relation to what you're dealing with. You then have to look at their words and acts according to all those together. ... But it takes time, practice and commitment. It took me constant training on the job for 25 years to get to this point. You have to be willing to be on constant training and open to new practices.

T HE STORY OF ADT INTRODUCES MANY CRUCIAL CONCEPTS THAT WILL BE FURTHER PROBED IN THE course of our journey. Among these are the image of cultures as intricate webs of competing meanings, the heuristic nature of cross-cultural interactions, the importance of context, and the necessary 'unDutching' element in such heuristic practices. The following chapter takes a closer look at the first concept in greater details, as it is one of the two core categories in the eventual proposed grounded theory. 


\section{Chapter 4}

\section{INTRICATE CULTURAL WEBS}

\section{A metaphor we live by}

The essence of metaphor is understanding and experiencing one kind of thing in terms of another. [...] We shall argue that [...] human thought processes are largely metaphorical. This is what we mean when we say that the human conceptual system is metaphorically structured and defined. Metaphors as linguistic expressions are possible precisely because there are metaphors in a person's conceptual system.

$$
\begin{array}{r}
\text { George Lakoff and Mark Johnson } \\
\text { Metaphors we live by } \\
1980: 5-6
\end{array}
$$

\section{$3334 \rightarrow 88$. .}

Metaphor is inherently paradoxical. It can create powerful insights that also become distortions, as the way of seeing created through a metaphor becomes a way of not seeing. [...] In recognizing theory as metaphor, we quickly appreciate that no single theory will ever give us a perfect or all-purpose point of view. [...] Some of the metaphors tap familiar ways of thinking; others develop insights and perspectives that will be rather new. Collectively, they demonstrate how we can use metaphor to generate a range of complementary and competing insights and learn to build on the strengths of different point of view.

Garret Morgan Images of organizations 2006: 5-6

\section{$.3334 \rightarrow 88$.}

The concept of culture I espouse [...] is essentially a semiotic one. Believing, with Max Weber, that man is an animal suspended in webs of significance he himself has spun, I take culture to be those webs, and the analysis of it to be therefore not an experimental science in search of law but an interpretive one in search of meaning.

Clifford Geertz The interpretation of cultures: selected essays 


\section{Reflection 9: Metaphorically speaking}

MOST people when embarking on a journey into a foreign land usually pick up a practical guide-book or are given one by a kind soul. I was no exception. When I arrived in Maastricht, a kind Dutch lady - who administered my study programme and whose Dutch name, Corien Gijsberg, was a challenge to my tongue - introduced us new alien students to a book called The UnDutchables. She even loaned me her own copy to read. For all practical purposes, that was my first introduction to the Dutch culture.

Since then, I have had extensive encounters with the Dutch culture, through academic and practical literature as well as first-hand. I was thus not surprised to learn from the many Dutch informants I talked to for this study that they too relied initially on those practical guidebooks and cross-cultural classroom trainings on how to live and work with the Thai when they first encountered Thailand and her people. What surprised me though, was a prevailing observation from most that, useful as these guidebooks are, there's no substitute for actual interaction with the Thai. Surprised I was, because I had up till then flattered myself that the exact same observation was unique only to my experience.

I hope that this study would reveal that those guidebooks and classroom trainings are but the very tip of the 'cultural iceberg', as a popular metaphor for culture would have it. Indeed, the more I delved into the question of what 'culture' really is and how to capture its flavour and essence, the more confused I became. Throughout the coding and theorizing process, I struggled with how best to articulate this well-known yet hard to elucidate concept. Countless coding memos on the concept of 'culture' I produced while I kept turning over the cases and their incidents and meanings in my analysis. The more I tried to explain to myself what culture meant to these informants and their stories, the more I felt like I was looking at a complex and complicated 'webs' of intertwining and inter-related elements - one tangle that, whenever I tried to untangle, would fall apart and lost its meanings instantly, not unlike if I were to jab at real spider webs. These intricate webs of meanings must be looked at as they are - intricate webs.

That was how I found a solution in the age-old power of metaphors. Once again, I took comfort when I discovered how there were many 'connoisseurs of metaphors' who have already made a great case for the use of metaphors in theorization. I felt reassured that my image of culture was nothing weird or unjustifiable.

AND so I came to detect from the case evidence an intriguing image of culture as a tangle of intricate webs of meanings. There my first core category was born - metaphorically speaking, of course. 
ULTURE IS SUCH AN ELUSIVELY COMPLEX CONCEPT THAT MANY SCHOLARS RESORT TO THE power of metaphor to capture its essence as they see it. Likewise, metaphor aids the elucidation of culture in my theorizing of this core concept. Specifically, grounded evidence in this study conjures up an image of culture as a multitude of intricate webs of competing meanings - an image that eventually serves as the underlying metaphor that underpins the first of the two core categories of the theory derived in this study ${ }^{17}$.

In conceptualizing culture, it is impossible to ignore the attempts made by a vast number of scholars to capture its essence. Yet, I must weigh all previous conceptualization of culture against the evidence I had in my hand from the cases and their meanings. As such, I must emphasize that the following interpretation of culture was based primarily on what this study evidence revealed to me, with support from existing literature in a selective manner. Only those elements from previous work that either confirmed or concurred with what emerged from my analysis of the field evidence were incorporated, merged, or extended in the following conceptualization of culture. To recap, my selective references to previous work should not be taken as a conventional literature review, but rather a theoretical sampling in grounded theory fashion.

\subsection{Images of culture: metaphors we live by}

The now classic book, Metaphors we live by, by Lakoff and Johnson (1980) has incited much interest in metaphor as a powerful conceptualization instrument. Despite inevitable critic and debate, their premise - that metaphor functions not only as a sheer linguistic device and in fact reflects the way the human mind works - has inspired a number of scholars to explore the theoretical power of metaphor further and deeper, not the least in the field of management and organization.

Most particularly, Garret Morgan put metaphor at the forefront of organizational studies when he set forth a provocative proposition that 'all theories of organization and management are based on implicit images or metaphors that lead us to see, understand, and manage organizations in distinctive yet partial ways' (Morgan 2006: 4, emphases added). Morgan's work elaborated the many insightful yet 'distorted' images of organizations, which, on the one hand, collectively provide a comprehensive view of what an organization is and how it works, and, on the other hand, individually distort our treatment of management and organization. Morgan's work serves to illustrate how metaphor affords an elegant yet delicate tool to scrutinize and explicate complex entities or concepts (Morgan 1986, Morgan 2006). Indeed his work has inspired an infant research theme on metaphor and organization which appears set to gain momentum (Cornelissen 2006a, Cornelissen 2005, Cornelissen and Kafouros forthcoming, Cornelissen, Oswick, Thoger Christensen, and Phillips 2008, Grant and

\footnotetext{
${ }^{17}$ The other core category is that of 'cross-cultural intelligence' or XCQ, a subject of Chapter 6.
} 
Oswick 1996, Heracleous and Jacobs 2008, Inns 2002, Tsoukas 1991). Most significantly, as argued by Cornelissen (2006b), the concept of 'metaphor as theory' or 'theory as metaphor' underlies a view of theorization as 'disciplined imagination', espoused by a prominent management theorist, Weick (1989) - a view adopted for the theorization, or rather theorizing, in this study. That is, culture is conceptualized in the grounded theory I propose through the use of one distinctive metaphor - that of $a$ tangle of intricate webs of competing meanings.

Looking at culture through a metaphorical lens is an approach by no means novel in cultural studies. Because culture is a complex and complicated concept that defies universal definition, any definition of culture is in essence a statement of what the definer perceives as its most important aspect (Kroeber and Kluckhohn 1963). Hence, history has shown that, in trying to define culture, many cultural theorists made use of the theoretical power of metaphor to illuminate their particular views of culture. As Morgan rightly cautioned, metaphor as theory is paradoxically discerning yet distorting: a particular choice of image magnifies one aspect of a concept it supposedly represents at the expense of overlooking or distorting some others. Yet, what Morgan proposed with regards to the many metaphors for organization applies also to the many metaphors for culture. That is, taken collectively as well as individually, the various images of a concept can 'demonstrate how we can use metaphor to generate a range of complementary and competing insights and learn to build on the strengths of different point of view' of that concept (Morgan 2006: 6, emphases added). In this fashion, the many metaphors for culture all help illuminate this very difficult concept.

Accordingly, although one distinct image of culture - the view of culture as a tangle of intricate webs of competing meanings - lies at the core of my theorizing in this study, this image also benefits from different views of culture expounded in earlier metaphorical representations. Specifically, the concept of intricate cultural webs as espoused here acknowledges and embraces the complementary and competing metaphorical meanings from the following three important sets of images of culture.

\section{Culture as the air or one's nose: the paradox of culture}

The first set of metaphors for culture focuses on its paradoxical nature, that is, its pervasive yet obscure presence. A. Lawrence Lowell introduced perhaps one of the earliest metaphors for culture when he lamented that an attempt to define culture 'is like trying to seize the air in the hand, when one finds that it is everywhere except within one's grasp' (Lowell 1934: 115).

There we have it: culture is like the air - as omnipresent as it is evasive. In the same vein, Gert-Jan Hofstede stated: 'Culture is like one's nose: everybody can see your nose except yourself' (Hofstede 2008). The images of culture as the air or one's nose emphasize its paradoxical nature of being obviously pervasive yet elusively imperceptible, particularly when, where and to whom it should be most obvious. The insight that individuals are usually unaware of their own cultures and their forces also 
brings home one very significant aspect of culture: culture exerts an unconscious influence and hence difficult to detect and unlearn.

Many informants in this study revealed the effect of this characteristic of culture in their reflections on their cultural identities. One recurring observation was that they often understood the cultures they belonged to most clearly when they must interact with people to whom those cultural traits were never a given - as if an encounter with other cultures acted like a mirror to reflect the image of their own cultures. For instance, in normal circumstances, most of the informants admit to have rarely been conscious of what denoted characteristic Dutch or Thai inclinations. The Dutch informants seldom noticed the comfort that came with the fact that 'yes' always meant 'yes' until they were puzzled by the Thai 'yes' that could mean anything from 'absolutely' to 'maybe' to 'no way'. The Thai informants seldom appreciated how their fellow Thais were quite astute at face-saving until they were hit with the Dutch axe of blunt remarks. As a reflexive note, I also found that I understood my own intricate cultural webs more profoundly in the course of this study. Particularly, I learned more about my two 'national' cultures - Dutch and Thai - through the attempt to juxtapose them side by side in this study. Apart from that, my other cultural webs, professional, biological, and others, were also revealed to me during the analytical process.

Thus, the paradoxical nature of culture can render cultural analysis rather problematic and elusive. Still, once we appreciate the paradox, it can aid our treatment of culture. Although we can hardly grasp the air in our hands, we certainly do breathe it; although we hardly see our own noses' in everyday situation, we certainly do make use of a mirror to reflect on our own images. This study, metaphorically speaking, attempts to breathe culture and devise a mirror to reflect on its significance.

\section{Culture as an iceberg or onion: visible and hidden cultural elements}

The two most widely known images of culture are ones that magnify its internal components as comprising of visible and hidden elements or layers, analogous to an iceberg or an onion. Although subtly different in their analogous exemplification, both models place particular emphasis on the significance of the 'hidden' elements of culture, espousing them as more difficult to discern yet exerting greater influence than the visible parts.

The iceberg model of culture - developed by two groups of scholars in organizational development studies, R. J. Selfridge and Sokolik (1975) and French and Bell (1972, 1999) - informs much of organizational culture research. According to this model, culture has two major elements: the visible one that accounts for only a small part, or the tip, of the whole 'cultural iceberg' and the hidden element which lies beneath and forms the very foundation of the whole. Usual examples of the visible, above the water part of the cultural iceberg are artefacts, written rules and explicit norms, whereas common examples of its hidden, under the water elements are assumptions, understandings, values, judgments, and beliefs. 
The other widely accepted metaphor for culture's internal components is the cultural onion model which informs much of cross-cultural management research (Adler 2002, Hofstede 2001, Schneider and Barsoux 2003, Trompenaars and Hampden-Turner 1998). Although there must be as many variations of the cultural onion as there are cultural theorists, all variants of the model share the following fundamental messages. First, culture manifests itself in diverse ways, shapes and forms - ranging from the most visible to the least fathomable - that may be likened to the many layers of an onion. The most easily seen and comprehended cultural layers are those on the outside, which include entities such as material objects, costumes, verbal and non-verbal languages, and architectures. The less visible inner layers comprise institutions and various cognitive attributes. Next, most theorists would go on to state that all these layers inter-related in a particular fashion. The most widely theorized relationship is that the inner layers influence the outer layers, and the innermost layer, or cultural core, is the key to all the influences.

From that point, though, the diverse cultural onion models vary in two respects: 1) what components make up the layers and what their relative positions are (particularly what constitutes the core and which dimensions it comprises); and 2) the exact nature of the onion's internal dynamics. Cultural core in particular became a topic that has received the most attention in cultural studies (Boyacigiller, Kleinberg, Phillips, and Sackmann 2004, Earley 2006, Holden 2002, Jackson and Aycan 2006, Leung, Bhagat, Buchan, Erez, and Gibson 2005, Usunier 1998). This topic is the heart and soul of the ubiquitous dimensional studies in cross-cultural management, which focus on defining, categorizing and scrutinizing cultural core and its dimensions. Schneider and Barsoux discussed a number of major views of cultural core, which they defined broadly as 'basic assumptions', and integrated them into one consolidated vision (Schneider and Barsoux 2003: 34-35). Notably, evidence from this study also concurred with some aspects in this view of culture, and thus the metaphor of cultural onion will be incorporated and extended to be one aspect of the core concept of intricate cultural webs, as duly elaborated in Section 4.2.

In summary, the iceberg and onion models of culture extend further the implication of its paradoxical nature by highlighting the fact that the more invisible part of culture, particularly the least fathomable (that is, the bottom of the cultural iceberg or the core of the cultural onion), while harder to discern, exerts greater consequence and hence demands meticulous theoretical scrutiny. The next set of metaphors, by contrast, moves beyond precise dissection and examination of cultural elements by zooming the conceptualizing lens on the question of how culture and its elements exert their influence - the nature of cultural force.

\section{Culture as software, mosaic, or webs: the complexity of cultural force}

Cultural theorists who seek to capture the working of cultural force eventually face with its enormous complexity. The question of how requires yet another view of culture - one that exemplifies not only a scrutiny of culture's internal elements, but also, 
and more so, a probe into how these elements exert their collective force. For this, various theorists selected diverse metaphors to elucidate their varying views.

The most acknowledged of such metaphors earned the title place of a wellregarded popular version of Hofstede's work which exploited modern day familiarity with computerization and looked at culture as 'mental program' or 'software of the mind' (Hofstede and Hofstede 2005). This metaphor portrays culture and its force as a collection of socially derived 'patterns of thinking, feeling, and acting' (Hofstede and Hofstede 2005: 3) that work to partially influence an individual's social act above and beyond the influence of that individual's personality. Hence, to extend the analogy, an understanding of the codes and coding of this mental programming 'indicates what reactions are likely and understandable, given [an individual's] past' (Hofstede and Hofstede 2005: 3). Under this view, Hofstede's five-dimensional model of national cultures and his detailed and extensive comparative analysis of a vast number of national cultures can be interpreted as results of the effort to decipher the codes and coding that make up the 'programming' of diverse national cultures of the world.

Looking at culture as socially derived software puts great emphasis on the cognitive aspect of cultural force: the image of software inevitably conjures up elements of deliberation, design, and structure. Such undertone well fosters the precision and orderliness needed in a quantitative approach of the burgeoning dimensional studies before and since Hofstede's work (Bond, Leung, Au, Tong, Carrasquel, Murakami, Yamaguchi, Bierbrauer, Singelis, Broer, Boen, Lambert, Ferreira, Noels, Bavel, Safdar, Zhang, Chen, Solcova, Stetovska, Niit, Niit, Hurme, Böling, Franchi, Magradze, Javakhishvili, Boehnke, Klinger, Huang, Fulop, Berkics, Panagiotopoulou, Sriram, Chaudhary, Ghosh, Vohra, Iqbal, Kurman, Thein, Comunian, Son, Ae, Austers, Harb, Odusanya, Ahmed, Ismail, deVijver, Ward, Mogaji, Sam, Khan, Cabanillas, Sycip, Neto, Cabecinhas, Xavier, Dinca, Lebedeva, Viskochil, Ponomareva, Burgess, Oceja, Campo, Hwang, D'souza, Ataca, Furnham, and Lewis 2004, House, Hanges, Javidan, Dorfman, and Gupta 2004, Inglehart and Baker 2000, Inkeles and Levinson 1969, Kluckhohn 1951, Leung and Bond 2004, Schwartz 1992, Schwartz and Sagiv 1995, Trompenaars and Hampden-Turner 1998, Welzel 2007), which explains why this metaphor captures the imagination of many scholars in cultural study.

A more recent metaphor for culture that also emphasizes elements of structure and deliberation, albeit with different interpretation, is one which looks at culture as a mosaic. The term 'cultural mosaic' has gained popularity as a metaphor for multiculturalism that embraces cultural diversity, as opposed to a long-standing image of multiculturalism as 'a melting pot', which endorses instead cultural convergence. Schwartz (1997: 263) examined this popular meaning of the mosaic metaphor for culture and traced its origin back to two Canadian scholars, Gibbon (1938) and Porter (1965,). The use of 'cultural mosaic' as a metaphor to promote cultural diversity, however, touches only at its most straightforward message.

Probing deeper, the image of culture as a mosaic has recently taken a more sophisticated conceptualizing flavour in cultural study. Notably, it constitutes the core thesis 
of Chao and Moon's proposition that the concept of 'cultural mosaic' be adopted to portray the complexity of cultural force, particularly at an individual level (Chao and Moon 2005). Applying concepts from chaos, complexity and network theories, they drew an analogy between an individual 'cultural identity' and a mosaic or 'a composite picture made up of distinct coloured tiles or miniature photographs' and proposed a taxonomy of cultural mosaic with three primary 'tiles' or categories: demographic (age, ethnicity, gender, race); geographic (climate, temperature, coastal/inland, urban/rural, regional/country); and association (family, religion, employer, profession, politics, avocations) (Chao and Moon 2005: 1129). The image of culture and its force as a composite mosaic of distinct and distinguishable 'tiles' or parts reinforces the complexity of culture as well as resonates with the conviction that such complexity, if not fully dissectible, is still founded upon strong structural and systematic elements and explicable with quantitative, positivist/post-positivist application of chaos, complexity and network theoretical tools. Most notably, as Chao and Moon (2005) elaborated, the cultural mosaic model aims to take a bottom-up orientation and focus primarily at the individual level of analysis, as opposed to the more common national or organizational levels of analysis. Arguably, this only represents a shift in orientation and does not reflect a strong departure from the common viewpoint in cultural study that each level of analysis can only be taken one at a time, one of the major issues that the next and last image of culture takes up as a point of departure.

That is, whereas the last of metaphors for culture in this set also highlights the complexity of cultural force, it paints a picture of such complexity that is a naturalistic creation and not compartmentalized system and hence embraces such complexity in a holistic rather than segmented fashion. The image of culture as webs of meanings, a metaphor more in tune with anthropological approach, conjures up an interpretation of culture that comprises discernible patterns but with no deliberate cognitive undertone. The image of multiple yet inter-connected 'webs' implies that such multitudes of patterns of meanings, despite its individual distinct design, are inherently interconnected in a distinguishable yet unpredictable manner. Analogous to physical spider webs, the pattern of cultural webs is naturally spun, so to speak. More, the image of inter-connected webs suggests that any attempt to dissect such webs would fundamentally disintegrate the whole picture, just like a jab at a tangle of spider webs would fundamentally alter, or even destroy, them altogether.

In her introduction to a reprinted edition of Benedict's Pattern of Cultures, Mead mentioned this metaphor for culture only in passing in the phrase 'the strength of the cultural web' but did not expound on it any further (in introduction to Benedict (1959: p. x)). Clifford Geertz, however, presented the image of culture as 'webs of significance' in a rigorous manner, and whose conceptualization very much informed the core category of intricate cultural webs that emerged in this study.

The concept of culture I espouse, and whose utility the essays below attempt to demonstrate, is essentially a semiotic one. Believing, with Max Weber, that man is an animal suspended in webs of significance he himself has spun, I take culture to be those webs, and the 
analysis of it to be therefore not an experimental science in search of law but an interpretive one in search of meaning.

(Geertz 1993, p. 5, italic added for emphasis)

Obviously, although software, mosaic and webs share a basic view of culture as socially constructed patterns of complex forces, each has its own underlying interpretation of how such patterns emerge and exert their influence. Specifically to this study, the evidence from the cases concurred most strikingly with the image of intricate cultural webs, underscoring its naturalistic nature, holistic essence, and interpretive force, thereby departing from the more popular inclination to view culture that focuses on its rationale characterization, departmentalized dissection, and cognitive governance.

Therefore, although my conceptualization of the culture concept also draws selectively on many elements in all of the above metaphors for culture, it is in Geertz's 'webs of significance' view of culture that this study and its findings find the most intimate connection. The next section elucidates this point in detail.

\subsection{Culture}

The concept of culture is itself a cultural product, as it is a mere mental abstraction. More, culture as it is used, defined and meant in contemporary social science discourse is a relatively young cultural product that only emerged in the late $19^{\text {th }}$ century. Prior to that, the word 'culture' carried only its original meaning of cultivation or tillage of land. The meaning of culture as a way of living had its seed in the Germanic intellectual heritage and was introduced into the English language by an English anthropologist, Sir Edward Burnett Tylor, in his book Primitive Culture (Tylor 1871). This anthropological meaning of the word took half a decade after Taylor's introduction to enter popular public awareness, marked by the addition of the 'new' meaning of the word culture in the supplement of the 1933 Oxford Dictionary (Kroeber and Kluckhohn 1963: 63). In short, anthropology gave birth to the culture concept as a subject of intellectual investigation.

The legacy of this anthropological root is evident in all cultural studies from any disciplinary perspective, including management and organization. Generally, the concept of 'culture' as introduced by anthropologists denotes the collective complex totality of a way of life which governs human beings' thoughts, feelings and conducts in such a way that it is possible to somehow distinguish one human group from another. Yet the exact meanings of culture are infinite; its formal definitions abundant. Ever since Kroeber and Kluckhohn (1963) published their meticulous cataloguing of 262 precise definitions of culture over four decades ago, we are even further than ever from unanimity on what culture actually is.

Thus, anthropologists have struggled with this perplexity ever since Tylor first introduced the concept. Sackmann's catalogue of the five major schools in anthropology gave a succinct bird-eye view of the variety and variants of theoretical efforts on the issue (Sackmann 1991, pp. 8-15). Notably, though, these variants have not been equally taken up by cross-cultural management scholars. 
Upon close examination, each major cultural anthropological school distinguishes itself in the details of how the cultural elements and their relationship are theorized and, most importantly, in what is identified as the analytical locus, or the core. That is, in anthropology, there are five distinct views of what culture essentially constitutes, or what is the locus of culture, namely, 1) 'personality writ large' (Benedict 1932, Benedict 1959, Mead 1949, Mead 1983), 2) an evolutionary system (Murdock 1940, Murdock and Provost 1973); 3) a value system (Kluckhohn 1951, Kluckhohn and Strodtbeck 1961, Kroeber and Kluckhohn 1963); 4) a web of symbols and meanings (Geertz 1993), and 5) a communication system (Hall 1989, Hall 1990a, Hall 1990b, Hall and Hall 1990). Each of these views first identifies, or implies, a different set of cultural elements and then translates the locus of analysis into a particular cultural core by exemplifying the core's unique characteristics and components.

The variety and variants in conceptualization of culture by diverse groups of management scholars echo this trend in anthropology, with each scholaristic group adopting primarily only one of the above five stances on culture and cultural analysis. Notably, the value system tradition is the single most received among management scholars, followed not too closely by the personality (psychological) and communication schools (Adler 1983, Boyacigiller, Kleinberg, Phillips, and Sackmann 2004, Sackmann 1991, Smith, Peterson, and Thomas 2008, Yeganeh and Su 2006). The dominant of the value system school led to another related trend in cross-cultural management research - the tendency to pin the analytical orientation on cultural core, namely on value system. This distinct trend motivated Holden to lament the dominant of the 'culture-as-essence' school of cultural study (2002: 55-58) - an issue I will duly revisit.

By comparison, the findings from this study suggest a stark departure from this general trend in management research in two aspects. First, as already introduced, evidence from the cases pointed to the significance of culture in relation not to value systems, but rather to networks of meanings. In effect, it concurred more with the fourth, much less popular tradition in the above list of anthropological heritages namely Geertz' tradition. Second, the evidence also suggested that our insight can also advance further by taking a departure from the attempt to dissect the components of cultural core and delve more into the interactive aspect of its operation - in brief, less focus on the what and more attention to the how.

Given the above introductory notes, I now present the concept of culture as emerged from the analysis of the cases and its meanings in this study.

\section{Culture: sense-making process and shared meanings}

As highlighted by the second set of metaphors for culture (the iceberg and onion models), most conceptualization of culture acknowledge the importance of the 'hidden' part of culture and place the locus of analysis on the 'subjective' or invisible aspect of culture rather than the 'objective' or visible manifestation of it, to use Triandis' broad taxonomy - subjective and objective - as a convenient and simple categorization (Triandis 1972). This view interprets the subjective, invisible part (however identified) to 
signify the totality of culture as well as each of its elements. In a broad sense, one single mutual agreement among all cultural theorists is that to understand culture, one has to focus on that aspect which is more than skin-deep - the innermost layers of the cultural onion, or the deepest parts of the cultural iceberg, as the two metaphors for cultures indicate.

But that is where the mutuality ends. Individual theorists then go their separate ways in identifying the exact subjective part of culture that they consider significance. The most common candidates for a locus of cultural analysis - by order of popularity, are: value, belief (or social axiom), basic assumption, (collective) personality, communication, knowledge and meaning. Among these, as earlier pointed out, value (and to some extent its close cousins, belief and basic assumption) has been by far the most preferred among cultural theorists (Adler 2002, Bardi 2001, Bond, Leung, Au, Tong, Carrasquel, Murakami, Yamaguchi, Bierbrauer, Singelis, Broer, Boen, Lambert, Ferreira, Noels, Bavel, Safdar, Zhang, Chen, Solcova, Stetovska, Niit, Niit, Hurme, Böling, Franchi, Magradze, Javakhishvili, Boehnke, Klinger, Huang, Fulop, Berkics, Panagiotopoulou, Sriram, Chaudhary, Ghosh, Vohra, Iqbal, Kurman, Thein, Comunian, Son, Ae, Austers, Harb, Odusanya, Ahmed, Ismail, deVijver, Ward, Mogaji, Sam, Khan, Cabanillas, Sycip, Neto, Cabecinhas, Xavier, Dinca, Lebedeva, Viskochil, Ponomareva, Burgess, Oceja, Campo, Hwang, D'souza, Ataca, Furnham, and Lewis 2004, Hofstede 1980, Hofstede 2001, Hofstede and Hofstede 2005, House, Hanges, Javidan, Dorfman, and Gupta 2004, Inglehart 1997, Inglehart and Baker 2000, Inglehart, Basanez, Diez-Medrano, Halman, and Luijkx 2004, Kluckhohn and Strodtbeck 1961, Leung and Bond 2004, Leung, Bond, Carrasquel, Muñoz, Hernández, Murakami, Yamaguchi, Bierbrauer, and Singelis 2002, Rokeach 1968, Rokeach 1973, Schein 2004, Schwartz 1999, Schwartz and Sagiv 1995, Trompenaars and Hampden-Turner 1998, Welzel 2007). One fundamental assumption and implication of this popular tradition is that a cognitive attribute (value, belief, or assumption) drives cultural dynamics, a tendency that is regretfully labelled as the 'culture-as-essence' fixation by Holden (2002: 55-58) as earlier introduced. Under the culture-as-essence conceptualization approach, the identified 'essence' or cultural core is then given prime, if not exclusive, attention, leading to meticulous dissection and rigorous examination of the core aspect of culture, be that value, belief, assumption, or social axiom. In effect, the 'culture-as-essence' approach is built on the faith that once we have identified what make up the core of culture, we can proceed to study any culture primarily by focusing on its core elements and once that is accomplished, we can so claim to know enough about that culture.

By contrast, cultural theorists who see locus of analysis as something other than mental attribute, tend to pay attention to a more interactive and interpretive aspect of culture, such as practice, communication, personality, behaviour, interaction, and sense-making, (Benedict 1959, Geertz 1993, Hall 1989, Hall 1990a, Hall 1990b, Jackson and Aycan 2006, Mead 1983, Triandis 1972, Triandis 1994). The theory proposed here shares an affinity with this second, hitherto less dominant, tradition of the above two major views of culture. Specifically, first, the proposed grounded theory focuses pre- 
dominantly on the interpretive and interactive aspect of culture and second, cultural core, though deemed noteworthy, does not constitute the single most significant aspect of culture. In fact, the theory goes beyond fixing its scrutiny purely on cultural core and pays greater attention to the actual totality of the interpretive and interactive dynamics of cultural force.

Thus, in this study, what emerged as the locus of culture was the sense-making process and the meanings its produces. Hence, culture is conceptualized as intricate webs of shared meanings that underpin two basic social processes: 1) interpretive processes, which involve the making sense of all that an individual encounters, whether material objects, social happenings, or mental concepts; and 2) behavioural processes, which comprise action, reaction, inaction, and interaction, depending on the meanings given to each object, event, or concept. Precisely, from evidence in this study, I conceptualize culture to be a shared way of being and sense-making that is unique to a distinct group of people and can distinguish that group from their counterparts.

The significance of the shared sense-making process and its eventual meaning in a cultural group as the heart and soul of culture recurred over and over in all cases in this study. The previous narrative of ADT presents a few examples of how the exact same entity was given entirely different meanings by different cultural groups, such as the disparity in how 'deadline' and 'mistake' were regarded differently by the Dutch and Thai employees. The significance resided not simply in the fact that the Thai were less conscious of time and more conscious of face, when compared to the Dutch, but more in how these different systems of meanings influenced their interpretation of 'deadline' and 'mistake'. The next two narratives will provide further examples to support this interpretation. Case CDW, for instance, will exemplify how cultural groups at various levels - national, professional, organizational and social - can be defined, identified, unified, and set apart, based on their distinct 'shared' manner of being and sense-making. The case illustrates that it was actually the tendency to give a specific meaning to an act, feeling or thought that distinguishes one cultural group from its counterparts. For example, at CDW, while engineers looked at cost-conscious attitude as an undignified mindset, accountants held the very attitude as their holy principle. Of course, this episode shows that the engineers at CDW held a value system that regarded quality over cost, whereas their accountant colleagues aimed more at value for money. However, what it illustrates more significantly is how these value systems only became significant when they influenced members of the two cultural groups to interpret the same practice in a particular manner that was shared by members of their own culture and distinct from that held by members of a counterpart culture.

From this perspective, culture influences how individuals think, feel and act, not in a one-to-one manner, but through their interpretations of the thoughts, feelings and acts of those they come into contact with. Yet surprisingly, with the notable exception of anthropologist Clifford Geertz (1993), most prominent cultural theorists have paid little, or virtually no, attention to this interpretive influence of culture, which this study 
has shown to be a vital aspect of cultural dynamics. Indeed, in the theory proposed here, this interpretive influence of culture is the prime, and supremely significant, force in any cross-cultural life because it fundamentally drives the directive influence. In other words, individuals think, feel, and act based on the meanings they give to the objects of their thoughts, feelings, and acts.

National culture, according to this conceptualization, is thus a way of being and sense-making that is unique to the group of people that belong to a particular nation (one way or another - either by birth, up-bringing or well-integrated naturalization). In this sense, Dutch people have a tendency to conduct their lives and gives meanings to things in a particular manner that are shared by their fellow Dutch countrymen. The same can be said of the Thai people. The degree, detail, and direction of the shared tendency may vary from one Dutch or Thai to another, but the shared way of 'making sense' does exist at a collective level. Directness, for example, is a strong Dutch trait, while face-preservation is a strong Thai propensity. As such, not only is a Dutch usually more inclined to be direct and a Thai to preserve face, each would also give different meanings to any direct or face-preserving acts or artefacts. A blunt Thai such as Khun Maleewan of ADT, for example, would still see differently from a Dutch how her fellow Thais could be insulted and lose face if confronted with Dutch directness. By the same token, a relatively gentle Dutch such as Khun Pim would still take the bluntness of a very direct Dutch like Khun Jeroen in a different manner than most Thais: he would not automatically relate it to the issue of face-preservation like many Thais would, at the very least. This is simply because the shared meanings of directness and facepreservation for the Thai and Dutch are two entirely different sets of meanings. ${ }^{18}$

Given this definition of culture, with its locus of sense-making and meanings, three significant characteristics of culture are derived. First, culture is a collective phenomenon; it is cultivated and shared by a group of people, horizontally (across roles and statuses) as well as transmitted vertically over time (through successive generations). Second, culture is cumulatively learned, often forgotten but hard to unlearn. Individuals are instilled cultural traits through successive experiences with a particular group they are a member of; culture is thus a social and not biological phenomenon. An ethnically Thai girl born to Thai parents but raised from childhood in the Netherlands by her adoptive Dutch parents would grow up to be basically Dutch and not Thai - an example of an occurrence that is not unheard of in modern day society. Yet, as a cumulatively learned force, culture is often forgotten such that individuals are seldom aware of their cultural traits even when these are exerting their influence: a notion already exemplified in the metaphors for culture as the air or one's nose. By the same token,

\footnotetext{
${ }^{18}$ It must be noted that what is broadly and qualitatively defined as 'shared tendency' here is in line with the notion of 'variation in value orientation' that was introduced by Kluckhohn (Kluckhohn 1951, Kluckhohn and Strodtbeck 1961) and explained in many literature in a quantitative manner using statistical terminologies such as modal personality (Inkeles and Levinson 1969) or the normal curve phenomenon of cultural homogeneity (Hofstede 2001, Hofstede and Hofstede 2005, Schneider and Barsoux 2003, Trompenaars and Hampden-Turner 1998).
} 
culture, being virtually ingrained in the subconscious like 'software of the mind' - to use the powerful metaphor proposed by Hofstede and Hofstede (2005) - is particularly hard to unlearn, or 'debug'. The term 'UnDutchables' is thus in fact applicable to any cultural trait: we are all unDutchable in varying degree. This is because while culture is unconsciously learned, it needs to be consciously unlearned if desired, and even when so desired, unlearning is not always entirely possible. To illustrate this difficulty using Khun Pim's experience, he reflected,

\begin{abstract}
I won't completely adapt and it's not a matter of 'willing' but more that I'm not able to adapt. For example, about the religion, here you're Buddhist and I respect the religion and appreciate many good things in Buddhism. But the way Buddhism is in the Thai people's hearts and souls - that can never be in mine. It's too difficult. I'm willing to, but I just can't. You were born and grew up with it.... This is not something you can learn. It's something you got used to or something you grew up with from when you're young.
\end{abstract}

Third, because any given culture is continuously being collectively formulated and reformulated by its current members, based on what has been formed by its past members, culture is dynamic, but not volatile. Dynamic regeneration does not equal volatility: unless cultural traits are relatively stable over time, a culture cannot retain its uniqueness. Nevertheless, culture does change, albeit through a long and subtle process. Not surprisingly, cultural change constitutes a rigorous field of research (House, Hanges, Javidan, Dorfman, and Gupta 2004, Inglehart and Baker 2000, Welzel 2007).

\title{
Cultural elements: a cultural onion model
}

Although my proposed view of the culture concept intentionally de-emphasize the significance of cultural elements and its core, it does make use of an appreciation of this aspect as part of the further probing into how these illuminate the interpretive and interactive issues. As a prelude, I now present a brief discussion of how I conceptualize cultural elements and its core.

For this, I again make use of the metaphorical cultural onion model because this image does indeed correspond well with what emerged from the grounded evidence in this study, with the added benefit of it being a popular choice among cross-cultural management scholars. Diagram 4.1 depicts a particular view of the cultural onion model that underlies the grounded theory proposed here, which is in essence an extended or embellished view of the popular cultural onion model.

This cultural onion model implies two main categories of layers, the top two dealing with the collective and organizational components of culture and the bottom two with its philosophical and interpersonal components. Thus, the first category comprises the material and institutional embodiments, so termed because they are either material objects or abstract mechanisms that embody shared cultural meanings. At the outer layer are tangible artefacts such as artistic objects (paintings, statues, music, dance, literature), architectural structures, language, and food. At the inner layer is the institutional apparatus that constitutes the social representation of the shared meanings, which includes all social mechanisms, rules, and systems created to regulate or 
organise such that members act and interact in conformity with the group's shared meaning. In a simple analysis, the outer layers embody the meaning of the inner layer. Architectural artefacts such as a mosque, church or temple, for example, can be interpreted to be an embodiment of the institution of religion in a particular culture, according to this cultural onion model.

Diagram 4.1: A cultural onion model

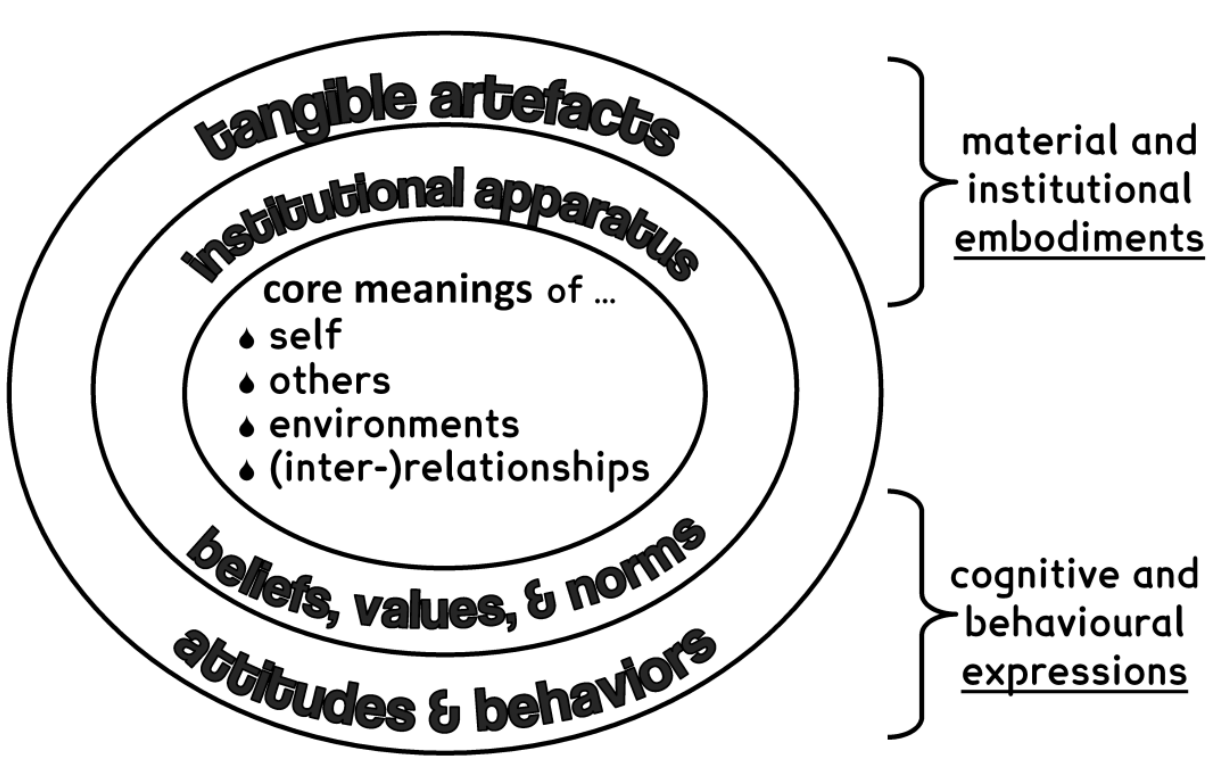

Note: Author's integration and re-conceptualization based on grounded evidence and relevant literature (Adler 2002, Bardi 2001, Benedict 1932, Benedict 1959, Bond, Leung, Au, Tong, Carrasquel, Murakami, Yamaguchi, Bierbrauer, Singelis, Broer, Boen, Lambert, Ferreira, Noels, Bavel, Safdar, Zhang, Chen, Solcova, Stetovska, Niit, Niit, Hurme, Böling, Franchi, Magradze, Javakhishvili, Boehnke, Klinger, Huang, Fulop, Berkics, Panagiotopoulou, Sriram, Chaudhary, Ghosh, Vohra, Iqbal, Kurman, Thein, Comunian, Son, Ae, Austers, Harb, Odusanya, Ahmed, Ismail, deVijver, Ward, Mogaji, Sam, Khan, Cabanillas, Sycip, Neto, Cabecinhas, Xavier, Dinca, Lebedeva, Viskochil, Ponomareva, Burgess, Oceja, Campo, Hwang, D'souza, Ataca, Furnham, and Lewis 2004, Geertz 1993, Hall 1989, Hall 1990a, Hall 1990b, Hall and Hall 1990, Hofstede 1980, Hofstede 2001, Hofstede and Hofstede 2005, House, Hanges, Javidan, Dorfman, and Gupta 2004, Inglehart 1997, Inglehart and Baker 2000, Inglehart, Basanez, Diez-Medrano, Halman, and Luijkx 2004, Kluckhohn and Strodtbeck 1961, Leung and Bond 2004, Leung, Bond, Carrasquel, Muñoz, Hernández, Murakami, Yamaguchi, Bierbrauer, and Singelis 2002, Mead 1949, Mead 1983, Rokeach 1968, Rokeach 1973, Schein 2004, Schwartz 1999, Schwartz and Sagiv 1995, Trompenaars and Hampden-Turner 1998, Welzel 2007).

The second category of layers comprises the cognitive and behavioural expressions that communicate the shared cultural meanings. At the outer layer lie the interpersonal expressions of the shared meanings: the behaviours - established, learned, and taken-for-granted patterns of action and interaction (verbal and non-verbal) that reflect or extend shared meanings - and the attitudes or evaluations (formed about objects, concepts, or behaviours) that are influenced by these shared meanings. At the inner layer lie the mental expressions of the shared meanings: the beliefs, which relate 
to the real, unreal, or surreal; the values, which determine what is good, bad, or neutral; and the norms, which delineate what is normal, abnormal, non-normal, or paranormal. Again, the outer layers express or reflect the meanings of the inner layers. The many versions of Thai smiles are thus various expressions of diverse beliefs, values and norms - including such notions as adherence to harmony, face preservation, avoidance of direct negativity, abhorrence of conflict, sensitivity to indirect or non-verbal clues, to list but a few.

Last but not least, it should be noted that the above representation of culture's internal components only attempts a pragmatic classification: it is not a strict portrayal of the complex abstract phenomenon under analysis. In actuality, the relationship among the layers is not a straightforward unidirectional correlation. To exemplify this point with an example, a wedding may embody marriage institution, which is itself an embodiment of various beliefs, values, and norms. At the same time, people's attitudes and behaviours in a particular wedding are influenced by yet many other beliefs, values and norms. In fact, the thrust of this observation foreshadows the concept of intricate cultural webs.

\section{Cultural core: core shared meanings}

At the core of the above cultural onion model lie the 'core meanings'. The conceptualization of cultural core as proposed here made selective, not integrative, note of prior view of cultural core cited earlier and compared them to what emerged from this study's evidence. The resulting conceptualization is relatively simple. What lies at the core of culture is simply a set of core meanings related to four fundamental concepts generally applicable to all cultural groups: the meanings of self, others, environments, and relationships and inter-relationships between and among the first three entities. First, the meaning of the self answers the simple question of what characterizes membership of a particular culture: 'what does it mean to be ... (Dutch, Thai, male, female, an engineer, an accountant, a sale manager, a Christian, a Buddhist, a Muslim, and so on so forth)'. The meaning of others signifies how individuals see and interpret the meaning of all other human-beings both within and outside their cultural groups. The meaning of the environments, physical as well as abstract, refers to how individuals see the world in all its facets, based on the shared meanings of their cultural groups. Thus it relates to what meanings a person gives to physical environments, such as natural surroundings, as well as abstract environments, such as time and space. Finally, the meaning of the relationship and inter-relationship between and among the first three basic entities refers to how individuals define the various relationships, based on the shared meanings of their cultures.

The concept of cultural core presented here, to repeat, is intentionally simple. It is meant to serve as a means for heuristic interpretation and not as an essentialist declaration as is usually the case in many cultural studies and theories. To explain, based on evidence in this study, there are as many definitive variations of the cultural core as there are cultures. As such, it is neither desirable nor feasible to give definite dimen- 
sions of this concept. The only comprehensible treatment of the cultural core is in the form of a very broad guideline that is general enough to lend itself to an analysis of any culture and cross-cultural interface, but in no way to be taken as an essentialist or universal law.

\subsection{Intricate cultural webs}

As earlier introduced, the first of the two main conceptual categories in the proposed grounded theory, the 'cultural webs' of meanings, is obviously not new, having been rigorously presented by Clifford Geertz (1993), whose conceptualization very much foreshadowed the expanded interpretation theorized here. It was thus both surprising and comforting to discover during theoretical sampling of literature that the image of culture as emerged from grounded evidence in this study was not too peculiar after all.

This image of culture as intricate cultural webs illuminates three related constructs - it characterizes first cultural unit (an entity), second cross-cultural interface (a process) and third, cross-cultural platform (a condition/context) in which this process transpires. In other words, a cultural unit is a set of intricate cultural webs that invariably operates in yet another superset of intricate cultural webs that make up a setting or context for any cross-cultural interaction. Thus, in any cross-cultural interface, intricate cultural webs stand as a shared property for the units, their operative process, and the context of that process.

\section{Cultural unit}

Most cultural studies delineate two basic classes of cultural unit: an individual and a group (Leung, Bhagat, Buchan, Erez, and Gibson 2005, Schneider and Barsoux 2003, Smith, Peterson, and Thomas 2008, Tjosvold and Leung 2003, Usunier 1998, Yeganeh, $\mathrm{Su}$, and Chrysostome 2004). However, such delineation is usually made to serve methodological purposes, particularly in studies that employ quantitative approach, which dictates precise definition of unit of analysis. Generally, the majority of studies on cross-cultural management focus on analysis of diverse individual cultural units as surrogates of the cultural groups that those individuals are members of, such as studying managers from different countries to extrapolate to the culture and management of the nations those managers belong to (Andrews and Chompusri 2001, Baker and Hess 2001, Chatterjee and Pearson 2002, Earley 1999, Hofstede 1980, Hofstede 2001, Ulijn, Rutkowski, Kumar, and Zhu 2005).

By comparison, the concept of cultural unit to be expounded on in this section serves primarily theorization purpose. That is, I now present my conceptualization of what a cultural unit is. The following conceptualization is thus again a fruit of theoretical sampling from the above body of literature, in conjunction with evidence from the cases.

Theoretically, a 'cultural unit' denotes a person or group whose individual or collective identity is shaped by one or (most likely) multiple cultures. Thus, as usually delineated, two main categories of cultural unit are first an individual and second a 
group of any fashion (such as a family, a clique, a department, an organization, a nation, and many others). In the theory proposed here, an individual is conceptualized as the basic cultural unit because every single individual is shaped by a combination of the diverse cultures he or she belongs to simultaneously. Thus, an individual comprises in essence a multitude of cultures within his or her cultural identity. A cultural unit that is a group then comprises many of these individuals who operate under a distinct set of shared meanings.

Evidence from this study suggests further that the delineation of one cultural group from another can then be drawn along two dimensions - those of cultural property and cultural boundary. Cultural property refers to the 'glue' that holds a cultural group together, a set of unique attributes that identifies the group as distinct from other counterpart groups. For example, national culture has a complex set of attributes, the most prominent of which include geographical border, nationality, legal legitimacy, a political system, an economic system, social institutions, and a history, among the most prominent. Cultural boundary then defines the limit of cultural property of a cultural group. For example, a national culture is bounded the simplest level by its geographical boundary, which is physical and definite. However, cultural boundary can also be abstract and flexible, such as a sense of nationalism or ethnicity that could also define national culture. Given this, boundary of a cultural group is inherently fuzzy and fluid.

Cultural group can then be further classified into two major categories - the formalised and the incidental. These two categories and their common sub-categories are summarily catalogued in Diagram 4.2.

First is the formalized category of cultural group, whose cultural property and boundary are relatively formal or 'formalized' and the sub-categories are inherently hierarchical in relation to one another. The formalized category of cultural group is often the first to be conceptualized, identified and elucidated in mainstream crosscultural management analyzes, as many cultural group types in this category are prevalent in business organizations. Schneider and Barsoux (2003), a good representation of such literature, discussed in detail what corresponds to the formalized cultural class here, which they named differently as 'cultural spheres of influence' (Schneider and Barsoux 2003: 51-79). Within the formalized category, the usual sub-categories are fairly straightforward as shown in the diagram in the order from higher to lower levels.

The second category of cultural group is the incidental category, so termed because the property and boundary of these groups are primarily social and the subcategories relate to one another in a fashion resembling an informal labyrinth rather than a formal hierarchy. The properties of the incidental cultural groups come from many possible inadvertent sources and the list in the diagram is only representative of the numerous such incidental cultural groups. 


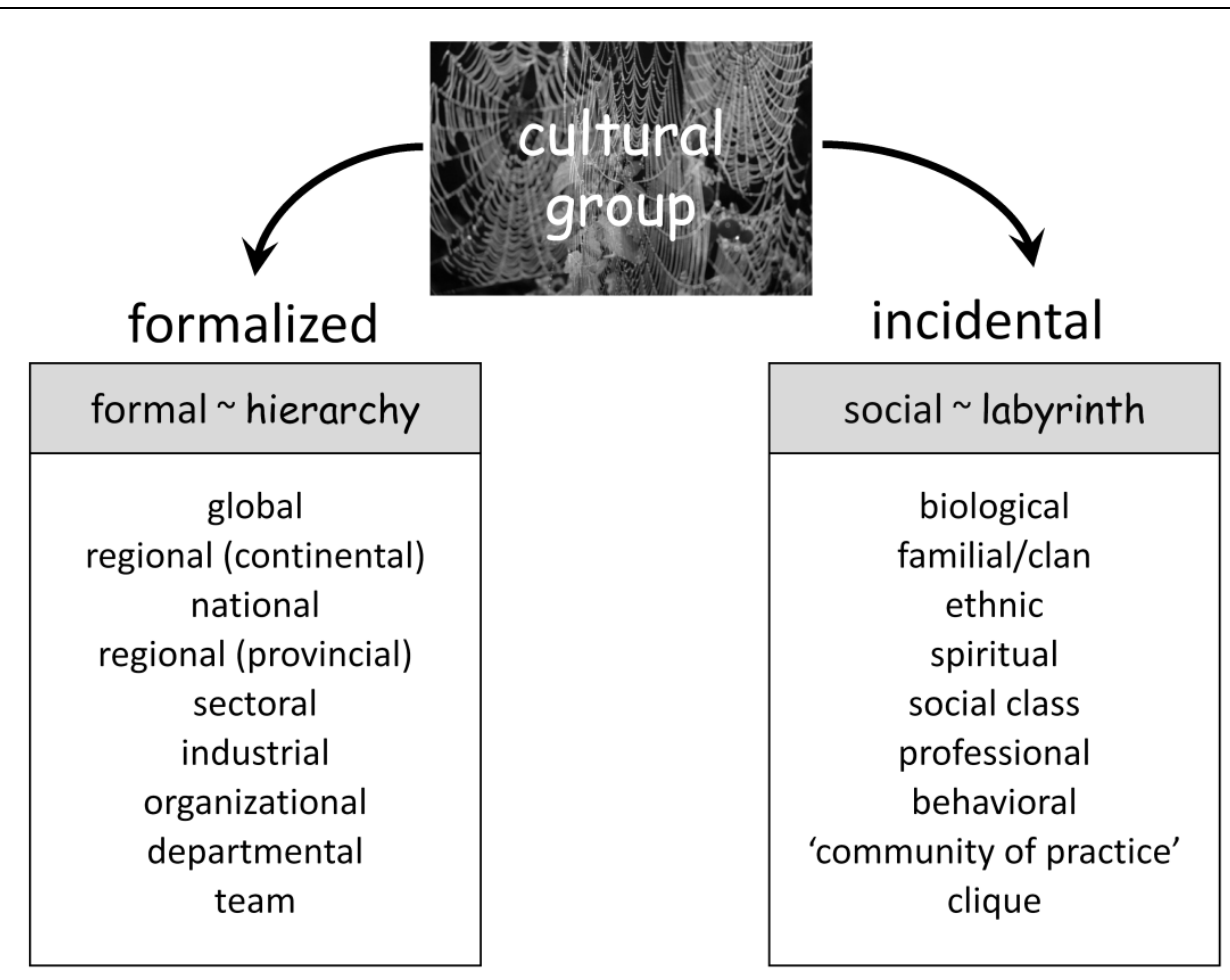

Note: Author's conceptualization based on grounded evidence and relevant literature (Adler 2002, Hofstede 2001, Schneider and Barsoux 2003, Trompenaars and Hampden-Turner 1998).

\section{Notes on the concept of cultural unit}

Three important notes regarding cultural unit are in order. First, as with the concept of internal cultural elements, the above categorization of cultural unit into two main classes of individual and group and the further subsets (formal and incidental) of cultural group serves only as a pragmatic examination of the concept of cultural unit and certainly not a definitive and exhaustive treatment of it. In particular, the list of all possible cultural group types is virtually indefinite and an attempt at or claim of an exhaustive list would defy practical comprehension. Secondly, the boundary and property of the many types of cultural group are neither mutually exclusive nor exact. Overlapping, intersection, and submersion of cultural groups are all too common, yet another condition that underlies the concept of intricate cultural webs. Again, this observation corresponds well with the notion of 'interacting spheres of culture' in Schneider and Barsoux (2003: 51-79).

Last and most importantly, the treatment of cultural unit as expounded here carries significant implications for the issue of 'level of analysis' - an issue that has been and is still being heatedly debated. As earlier mentioned, in many cultural studies, particularly the ubiquitous dimensional research, a precise identification and treat- 
ment of the exact cultural unit under study bears significant implications on the interpretation and validity of the study results. This relates primarily to the constraint of quantitative approach, for which unit of analysis must be exactly defined and properly operationalized. Debates on this issue centres on the validity of an interpretation of data or results gathered at an individual cultural unit when applied to or projected on various cultural groups that those individual are theorized and operationalized to belong to. For example, one prevalent debate concerns the validity of the results of many studies on national cultures that are based on survey of individuals (Hofstede and McCrae 2004, Maznevski, Gomez, DiStefano, Noorderhaven, and Wu 2002, Peterson and Castro 2006, Smith 2004). However, this study and its subsequent grounded theory, being qualitative in nature, needs not be constrained by the precision required in quantitative approach and hence adopts a different treatment of this issue of level of analysis. Specifically, for this study and its resulting theory, the unit of analysis issue itself represents yet another set of intricate cultural webs, rendering simultaneous look at different levels of analysis not only possible but essentially imperative.

To begin with, the relevant cultural units for this study were at two levels: 1) the individual actors (Dutch and Thai employees) in each case, and 2) the case itself as a unique cultural group. Whereas the second level was the core cultural unit of the theorizing effort - which focused on cross-cultural intelligence that was manifested when practiced via interactions of individuals that formed a particular group in a particular cross-cultural setting - the study must also look at the individual unit of analysis at the same time. More, other relevant cultural groups in the case, each representing diverse levels of analysis - such as the companies (organizational), the countries (national), the industries (industrial), the profession (professional) and so on - must also be included in the analysis when relevant. This mandatorily simultaneous treatment of multiple levels of analysis recurs time and again in various sections of this manuscript, again reflecting and responding to the very nature of intricate cultural webs.

\section{Intricate cultural webs}

As the first of the two core theoretical categories of the grounded theory proposed here, the significance of intricate cultural webs concept is threefold: it characterizes three theoretical constructs: first, a cultural unit (an entity), second, a cross-cultural interface (a process), and third, the platform under which the units operate and the process transpires (a context).

\section{Intricate cultural webs as cultural identity of a cultural unit}

Intricate cultural webs are the fundamental property of any cultural unit, whose identity is made up of diverse cultural traits which simultaneously influence the operation of that cultural unit. Thus, 'cultural identity' refers to the combined cultural forces within a cultural unit, whether an individual or any type of groups - be it team, organization, nation, or others. Because a cultural unit, even if an individual, invariably belongs to or is made up of more than one cultures, its property can be depicted, at the 
simplest level, as a cluster of many cultural onions (as earlier expounded). Such an image of diverse cultural onions moves the conceptualization of cultural identity into a new level of complexity - that of complex webs. Hence the property of a cultural unit is better characterized as intricate cultural webs - the key concept of this chapter and the first of the two core category of the grounded theory from this study. That is, a cultural unit represents a set of complex webs of cultural layers of the many cultures to which it belongs, of which it is made up, and by which it is constantly being influenced. ${ }^{19}$ The dynamics of intricate cultural webs in a cultural unit reflects how its cultural identity is constantly influenced and reformulated by multiple cultural forces. That is, as that unit continues to operate, its cultural webs are forever spun, metaphorically speaking.

The cases in this study illuminate two different types of such dynamics: repositioning of cultural stimulus and transformation of cultural profile. I conceptualize the distinction between repositioning and transforming with the use of an analogy to the distinction between movements along a curve v. shifting of the entire curve, to borrow an apt economic analytical tool.

Repositioning of cultural stimulus within a cultural unit is akin to the movement along a curve (e.g. demand or supply), which means the integral identity of the cultural unit is not 'transformed' in any fundamental way and how the unit makes sense, gives meaning to objects or concepts, and acts upon those meanings is merely a response to context. This is the case when a cultural unit operates in a situation under the sheer command of a particular culture (or sets of cultures) that the unit belongs to. For example, how Khun Kees, the CEO of ADT, as an individual level unit, made sense of and reacted towards the crisis incident was based primarily on his professional cultural identity as the top executive. His interpretation of the situation (that the Thai did not learn from their 'mistakes') was based primarily on his Dutch working culture (mistakes are nothing to be ashamed of and ignored, but something to be guilty of and learned from). Then, in another incident, when Khun Kees accepted how Thai people could behave in their own ways as long as it did not damage business results, he was, in a sense, refocusing his cultural reference to the context, that is, from a Dutch operating in the Netherlands to a Dutch operating in Thailand.

Transformation of cultural profile, by comparison, is analogous to the shifting of an entire curve, up or down, or even in its elasticity property. In this case, there is a fundamental transformation in the integral identity of a cultural unit. The case of Khun Pim of ADT, again as an individual unit, who eventually wanted to settle down in Thai-

\footnotetext{
${ }^{19}$ Theoretical sampling of existing literature has led to a discovery of yet another stream of theorization in social psychology which corroborates some elements in the above concept of cultural identity. These are the work that examine the broad theme of a general theory of the self (Stets and Burke 2000), which comprises the closely related identity theory and social identity theory (Hogg, Terry, and White 1995, Stets and Burke 2000, Stryker and Burke 2000). In this sense, social psychology theories of identity also corroborate the concept of cultural identity as espoused here (Ashforth and Mael 1989, Burke 2003, Burke 2006, Tajfel and Turner 1985, Worchel, Morales, Paez, and Deschamps 1998).
} 
land, provides an illuminating example. In his case, he could no longer go back to live and work in the Netherlands (or the West) without feeling a certain discomfort, something that never happened prior to his cultural transformation. In other words, his national cultural profile had been transformed from a Dutch to a hybrid Dutch-Thai. This signified not just a shifting of cultural stimulus (vis-à-vis context) within his own intricate cultural webs but that the entire pattern of his cultural webs had changed. This transformation was not dependent on a situation but rather evolved over a period of time before Khun Pim assumed an entirely different national cultural profile altogether.

\section{Intricate cultural webs as property of a cross-cultural interface and platform}

Cross-cultural interface is any process that transpires in a cross-cultural platform or context. As stated earlier, both cross-cultural process and its context share the mutual characteristic of involving multitude of intricate cultural webs.

Cross-cultural platform is a social setting or context that entails two or more competing cultures. By 'competing cultures' is meant different cultural units of the same level and type, for example, Dutch v. Thai (national culture), male v. female (gender), working v. middle classes (social), and so on. As such, a particular cross-cultural environment always has the undercurrent of a specific set of multiple meanings of the same concept or object competing within a specific context. For example, Dutch and Thai views of time are distinctly different and when the Dutch and Thai have to work together with time as a benchmark, their different views of time come into contention with each other, as was the case of the meanings of 'deadline' at ADT.

What is thus critical in an analysis of cross-cultural interface is to identify the key competing meanings of the phenomenon under scrutiny, that is, which 'crossing of meanings' is of critical influence in a particular context and for the particular process that transpires in that context. This exercise emerged to be one distinct element in the cross-cultural heuristic - the core of cross-cultural intelligence - which will be presented in Chapter 6.

\section{Properties of intricate cultural webs}

To recap, intricate cultural webs characterize cultural unit, cross-cultural process and cross-cultural condition, three constructs that share one critical property: all three are made up of a multitude of cultural webs - complex networks of interlacing cultural layers and levels. Such cultural webs themselves have one key property: the exponential complexity that inspired my choice of the metaphor 'intricate cultural webs'. Even in simple situations like one interaction between a Dutch manager and a Thai subordinate of a particular Dutch firm in Thailand, the entire event actually operated under the forces of a multitude of cultures and sub-cultures, all of which comprise many complex layers. Thus, every analysis must effectively examine multiple, complex, and complicated cultural webs and all their forces. 
Such complexity results from the following properties. First is the presence of countless identities that are intertwined from many layers and levels. Second is the sheer fact that these many identities are always in constant flux, invariably developing as part of the larger historical and social process that involves infinite cultural units with multiple cultural identities. Metaphorically speaking, all cultural webs at all levels are forever being spun and re-spun such that to find a pattern in this constant flux is extremely challenging, if not impossible. Third, every cultural unit, cross-cultural process and its condition operate and transpire within a tangle of cultural webs and is subject to the interaction effect of cultural forces. Identifying how each cultural unit or cross-cultural situation is influenced by what element(s) from which of the multitude of cultures is difficult because in most cases the influence is not from one single effect but, to borrow a quantitative term, from an interaction effect. For example, when a female, Thai, newly rich (formerly lower class) engineer working for a Dutch male boss in a Dutch-owned company in Thailand (aka Khun Maleewan of ADT) acted in a certain way in one particular situation, what can serve as a benchmark for analyzing her action, reaction, or inaction? It is extremely difficult, if not impossible, to derive a finite formula to predict that, for instance, she did $X$ because she is female, $Y$ because she is Thai, and $Z$ because she was reacting to a Dutch boss. Even in only one instance, the particular actor had all these qualities and more, making it virtually impossible to ascertain the exact identification of cultural forces that triggered her one action, let alone pinpointing how the interaction of such forces exert their 'combined' influence.

Given the above exponentially complex nature of cultural webs that underlie a cultural unit and cross-cultural life, any theorizing on cross-cultural issues is constrained by one significant limitation. Because cross-cultural analysis inherits this complexity, the more the cultural units, layers, and levels in the analysis, the more the complexity grows exponentially. As this study results show, even the simplest of real-world situations is actually made up of practically uncountable numbers, and the often unaccountable presence, of cultural units and their forces. The significance of this intractable complexity and non-deterministic nature of cultural webs exerts a great influence on the nature of cross-cultural intelligence, as will be further expounded in Chapter 6.

\subsection{Core meanings: Dutch and Thai}

As a substantive exercise, I now turn the theoretical lens to focus on the crossing of the Dutch and Thai cultures. This section introduces the underlying webs of meanings in these two cultures by identifying parallel core meanings of the two national cultures. For this purpose, my earlier elaboration on a cultural onion model and my theorized 'cultural core' serve a useful purpose. Metaphorically speaking, I now turn the theoretical lens to focus only on one 'slice' of culture - that of national culture. Diagram 4.3 summarizes core meanings in the Dutch and Thai cultures, as detected in this study evidence, using the framework provided by the cultural onion image. 
Even at cursory glance, the contending nature of the two sets of meanings is interestingly evident. Not only do the two sets of meanings differ, they differ in quite a stark fashion. This is the first signal that the undercurrent of national cultures in the cross-cultural context under scrutiny has a high potential to exert consequential influences, because it provides a rich ground for competing meanings. This observation touches on yet a similar issue in mainstream cross-cultural study, namely the issue of cultural distance - a research area that aims at development, application, and interpretation of quantitative measurement of the extent to which cultures (particularly national) differ from one another and what that 'distance' means in cross-cultural interaction. Study on cultural distance has become a rigorous area of research in crosscultural management (Shenkar 2001). The observation in this study, however, remains qualitative and hence the main interpretation of the starkly different nature of the core meanings in the Dutch and Thai cultures suggests only that there appears to be rich ground for competing meanings when the two cultures cross.

\section{Diagram 4.3: Core meanings: Dutch and Thai cultures}

\begin{tabular}{|c|c|c|}
\hline Meaning of ... & Dutch & Thai \\
\hline self & $\begin{array}{l}\text { is defined primarily by self. } \\
\rightarrow \text { You are what you make of yourself. }\end{array}$ & $\begin{array}{l}\text { is defined primarily by others. } \\
\rightarrow \text { You are what people make of you. }\end{array}$ \\
\hline others & $\begin{array}{l}\text { is defined by their acts in a situa- } \\
\text { tion, in relation to what contribu- } \\
\text { tions those acts make. }\end{array}$ & $\begin{array}{l}\text { is defined by their roles in a } \\
\text { situation, in relation to how } \\
\text { they fulfil those roles. }\end{array}$ \\
\hline environment & $\begin{array}{l}\text { is a given, to be explored and } \\
\text { exploited. } \\
\text { Nature: is functional and manageable. } \\
\text { Time: is valuable and must be used } \\
\quad \text { efficiently. } \\
\text { Space: is scarce and must be used } \\
\quad \text { efficiently. }\end{array}$ & $\begin{array}{l}\text { is a gift, to be thankful for and } \\
\text { revered. } \\
\text { Nature: is sacred and animate. } \\
\text { Time: serves merely as a point of } \\
\text { reference. } \\
\text { Space: is sacred as it is part of } \\
\text { nature. }\end{array}$ \\
\hline (inter)relationship & $\begin{array}{l}\text { is defined by relative contribu- } \\
\text { tions to a situation. }\end{array}$ & $\begin{array}{l}\text { is defined by relative roles in a } \\
\text { situation. }\end{array}$ \\
\hline
\end{tabular}

Note: Author's theorization based on grounded evidence and relevant literature (Atmiyanandana and Lawler 2004, Baker and Hess 2001, Earley 1999, Hampden-Turner and Trompenaars 1993, Hofstede 2001, Holmes, Poonprasit, and Komin 2006, Holmes and Tangtongtavy 1997, Klausner 1993, Komin 1989, Komin 1991, Lawler and Atmiyanandana 2004, Lawler, Siengthai, and Atmiyanandana 1998, Lawrence 1991, Mulder 2000, Redmond 2002, Segaller 2005, Siengthai and Bechter 2004, Siengthai and Bechter 2005, van der Horst 1996, Vossestein 2004, White and Boucke 2006).

Further, I must point out that the issue of cultural distance implies also the other side of the coin. That is, meanings in different cultures can be either 'competing' or 'congruent' in nature. The detected competing nature in the Dutch and Thai sets of core meanings provides a good example of competing meaning. Yet, in some other 
pairs of cultures, where 'cultural distance' is less wide so to say, the sets of core and other meanings may instead reveal more congruence rather than opposition. I will revisit this issue and extend its implications further as part of the elaboration of crosscultural heuristic in Chapter 6. For now, the relevant observation is that the Dutch and Thai sets of core meaning appear to reveal competing more than congruent nature when set side by side.

\section{The meaning of 'self'}

First is the meaning of self, or in other words, the meaning of one's existence. In the Dutch culture, this is defined primarily by the individual himself or herself, meaning 'you are what you make of yourself'. Not surprisingly, the Netherlands scored very high in Hofstede's individualism index - with an index of 80 and a rank way at the top 4-5, on par with Canada and only surpassed by the US, Australia and Great Britain (Hofstede 2001, p. 500). Yet, the Dutch's view of self has its unique quality, which is in line with what has been theorized to be a major common Dutch trait: egalitarianism (Hampden-Turner and Trompenaars 1993, van der Horst 1996, Vossestein 2004). Van der Horst's explication of the Dutch egalitarianism concurred with much that was also seen in the cases in relation to how the Dutch characters interpreted their own existence.

According to van der Horst, the Dutch version of egalitarianism has its particular flavour, '[i]t advocates cultural individuality but not personal egoism' (van der Horst 1996, p. 57), which distinguishes the Dutch individualism quite starkly from other strands, such as the American, to cite the most obvious example. For the Dutch, the meaning of oneself is first and foremost a self-responsibility before it can turn into a right or privilege. You are responsible for your own significance but that does not mean that you are the only one that matters. Everybody is subject to this one responsibility and that is what makes for equality. You have to earn your meaning, in the Dutch eyes; you are not simply born deserving it. Again, you are what you make of yourself, in a nutshell.

This egalitarian individualism could be detected in many of the Dutch characters in the case. Broadly speaking, when the Dutch informants in the study looked at themselves, they tended to talk in terms of what they had accomplished and had yet to pursue and what their duties and tasks were. They seldom looked at themselves in terms of what other people thought of them. If they did so, they seemed to do it more out of curiosity than anxiety. Khun Kees of ADT, for example, carried a view of himself as the CEO that was more related to what he had done to earn that function and what he must continue to do to remain in that function. He even felt embarrassed when people treated him in a brown-nosing way simply because he was the CEO. To him, it was a CEO in him, and not him as a CEO, that made him matter.

The Thai, by contrast, tend to define the meaning of self based primarily on how they think other people surrounding them make of their existence. It is not that they do not take their own idea of themselves into account. They do, but only after and in 
relation to what they deem to be the idea other people have of them. Again, not surprisingly, Thailand scored in the opposite direction to the Netherlands in Hofstede's individualism index - an index of 20 and ranked way towards the bottom half at 39-41. Many analyses of the Thai culture indeed unanimously classified it as a 'collectivist' society, where collective thinking exerts decisive forces on individuals (Atmiyanandana and Lawler 2004, Holmes and Tangtongtavy 1997, Komin 1991, Mulder 2000, Redmond 2002).

Interestingly, Komin reported her quantitative survey results to show that ' $\mathrm{t}] \mathrm{he}$ Thai are first and foremost ego oriented, characterized by the highest ego value,' (Komin 1991: 133), which seems to contradict what is espoused here. Yet, at closer look, her interpretation of the ego-orientation in fact described exactly how the Thai 'ego' is shaped in a collectively oriented way, because by 'ego oriented' she meant highly conscious of self-image. In her very telling words, "[t]he "face" is identical with "ego" and is very sensitive' resulting in 'a tremendous emphasis on "face" and "ego" [such that] preserving one another's "ego" is the basic rule of all Thai interactions' (Komin 1991: 135). Ego is, in others words, defined by what is in the open, through social interaction. Komin then went on to relate what she described as ego-orientation to three other prominent Thai values: face-saving, criticism avoidance, and being considerate, or the notion of ' $k$ reng jai' ${ }^{20}$. As such, for the Thai, self or ego is what is out there in the open, defined and revealed by how others treat and define you. You are what people make of you, in a nutshell.

Holmes and Tangtongtavy (1997) gave an insightful view of how the Thai would behave based on other people's view. They theorized on the exact nature of 'others' that the Thai take into account under the framework of 'the three circles of Thailand' (Holmes and Tangtongtavy 1997: 39-44), which basically classified the three spheres of social circles from the most intimate one - the family circle, to the non-family but important one - the cautious circle, to the negligible one - the selfish circle. They elaborated on how Thais would behave differently when operating in each of these circles. Their observation actually sheds interesting lights on how the Thai define the meaning of themselves. That is to say, how a Thai takes into account what other people think of himself or herself, depends very much on from which of these circles those others belong to. How a family member, or someone you consider as virtually your family, makes of you is meaningful but can be taken for granted. Your family must always think well of you and even when they do not, they are often ready to help you work on

\footnotetext{
20 This Thai term cannot be avoided here because the notion of 'kreng jai' defies any accurate translation. There is no one single word in the English or Dutch languages that actually conveys all that 'kreng jai' means in Thai. 'Considerate' in English and 'zorgzaam' in Dutch are the closest; yet they do not convey precisely what 'kreng jai' means in Thai culture. In brief, to exercise the spirit of 'kreng jai' means to do things in a manner that causes the least discomfort and preserve the face and dignity of all parties involved. It motivates the tendency to avoid any act or word that could harm someone's feeling, put him or her in an awkward position, cause him or her to lose face, or create an inconvenient or uncomfortable feeling of any kind. This usually results in an avoidance of direct confrontation and open negativity altogether.
} 
a new meaning that is more desirable. Those others from the cautious circle, however, are ones whose notion of you must be cautiously cultivated and harvested. These are the people you interact with on a social or formal basis, at work, social functions, and so on. How the views of these others would contribute to the meaning of your existence, in turn, depends on who they are, to you. How your boss sees you, for example, would contribute much to who you are at work. If he or she thinks well of you, then you are significant in your work function. The same can be said of your teachers, friends, colleagues and so on. Finally, the negligible others are those from the selfish circle, the circle where you can totally define your own ego without paying much attention to the views of these insignificant others. These are people who have no lasting influences on your life and as such, how they make of you have no influence on the meaning of your existence.

Most of the Thai characters in the cases viewed themselves first and foremost based on the way they perceived how the three circles of others think of them. Khun Maleewan of ADT, for example, was very proud to hear that her Dutch bosses thought highly of her and did not care greatly that some of her subordinates thought of her as being too blunt. Actually, according to her, that was a good sign, because it was proper that they respected and revered her. Khun Busara of CDW in the upcoming case, expressed discomfort when she discerned that some of her Thai colleagues had thought of her as the Dutch bosses' 'pet' because she still wanted to be part of the Thai social circle and did not like the derisive element associated with that picture of herself. She described how she needed to correct this image, even though she did not think of herself in that way at all.

The episode concerning mistake at ADT provides a good illustration of how the Thai define the meaning of themselves. For the Thai employees, the company and their bosses were 'family' and the mistake was seen as a loss of face to the outer cautious circle, the customers, whom they needed to be most careful with. Their reasoning could be as follows. The customers must always think well of you, else you would lose your face, and your business. How the bosses thought of you and how you acted in this particular incident was an internal affair that could be easily resolved within the family. Thus, you must take quick action to remedy the customer's negative view first whereas internal affair could be taken care of later.

\section{The meaning of 'others'}

The meanings of others in the Dutch and Thai cultures are understandably related to the meaning of self as described above. At the most obvious level, how the Dutch and Thai give meaning to others, is a kind of mirror image of how they give meaning to themselves, although the process has its own slant and emphasis.

The Dutch define the meanings of others by their acts in a situation and in relation to what contributions those acts make to the situation. In a way, this is rooted in the egalitarian spirit of the Dutch culture - each person is judged based on how he or she is and acts, individually. It also relates very much to the Dutch utilitarian orientation as 
identified by van der Horst (1996). For a Dutch, those whose acts contribute positively to a situation are one whose existence bears valuable meaning and vice versa. Thus the Dutch take people based on what they do and the contributions they make. The Dutch characters in the case invariably talked of other people in terms of their actions and contributions, and rarely in relation to their status, character, or personality.

The Thai, on the other hand, are first and foremost concerned with what role a person has and how he or she fulfils that role. The meaning of other people is first defined by looking at who they are in a situation. The role of each person in a situation has first to be established before one know how to address and interact with every individual one meets. This resonates very much with how Thailand scored fairly high in Hofstede's power distance index, with a score of 64 and ranked 21-23 towards the top. There is, however, more than a simple rule of hierarchy in the Thai webs of meanings, which underlies how a Thai gives meaning to other individuals. Again, the three circles of Thailand concept provides a good explication. The Thai see meaning in others, first and foremost, based on which circle those others belong to. Within each circle, there are a set of roles, each of which with its own specific rank, status, behaviour, contribution, and so on, or in one word, the total 'meaning' of a person in that role. A role is then defined in a rather complex fashion, involving initial birth condition, age, gender, education, social status, achievement, and all else that make up a person.

Once roles are established in a situation, the Thai would then see others based on how they fulfil their particular roles. Certain expectations of character and behaviour are always associated with each role. In general, the higher in rank a role is, the more superior quality is expected, in all aspects. The four top roles in the Thai society at large, by order of superiority, are 1) the royalty, 2) the monk, 3) the teacher, and 4) the doctor (Baker and Hess 2001, Chatterjee and Pearson 2002, Earley 1999, Holmes, Poonprasit, and Komin 2006, Holmes and Tangtongtavy 1997, Klausner 1993, Lawler and Atmiyanandana 2004, Lawler, Siengthai, and Atmiyanandana 1998, Mulder 2000, Siengthai and Bechter 2005). That a king can do no wrong and is regarded almost like a living god, reflects this fundamental meaning of role in the Thai culture. Still, role definition is always set against role fulfilling, and when the two do not correlate in a person, the meaning of that person diminishes significantly. There is a Thai saying 'bhun tam karm tang' which translates as 'one is born with a predestined fate which is then shaped in this current life by one's act'. The meaning of act here, however, is more in line with the meaning of karma and not a mere action. The spirit of this saying underlies how the Thai give meaning to others, in the sense that once a role is established, one must act to fulfil it accordingly, if not, one's meaning will depreciate.

In the work place, for example, a boss is expected to be superior in all aspects of character and behaviour. He or she is supposed to know best, act kindly but decisively, make good decisions, lead with confidence, take responsibility, be morally and ethically sound, and so on so forth. If a boss does not fulfil these expectations, a Thai subordinate tends to think less of him or her. A subordinate, by comparison, has a different set of expectations to fulfil. In general, a subordinate does not need to know 
best but must follow order well and be loyal to the boss without a speck of doubt. This view of boss and subordinate permeated in all the Thai characters in the cases, in varying degrees. Khun Maleewan for example, expressed a clear opinion that she preferred to have Khun Pim be regarded as a superior and kind boss, who would never hurt the employees, because in her eyes, that was the role he needed to fulfil as the top boss of ADT Thailand or, figuratively speaking, the father of the family, if he were to be regarded well by the Thai employees. Many of the Dutch characters, on the other hands, expressed great frustration that their Thai employees seemed to lack a sense of initiative and only waited to follow orders.

\section{The meaning of 'environment'}

The Dutch and the Thai look at the environment, again, in a starkly opposite way. For the Dutch, the environment is simply a 'given' whose value must be explored and exploited. By explore is meant a practical survey to evaluate the situation and by exploit is meant to make the best possible use of what is given. This applies to all aspects of environment.

Thus to the Dutch, natural environment serves a functional purpose and must be managed to the best of its potential. The famous Dutch dykes and other ingenious ways to make use of land and water are testimonials to this spirit and often used to characterize the Dutch utilitarian view of their physical environment (Hampden-Turner and Trompenaars 1993, van der Horst 1996). The Dutch treat abstract environment in the same fashion. Time and space are truly scarce resources to the Dutch, serve functional purposes, and must be managed and used efficiently. Many of the Dutch characters in the case expressed frustration at how the Thai never took appointment seriously and always showed up late. Punctuality is a virtue in the Dutch culture because time is precious and must be well observed. In all the five companies investigated (including the two vignettes), the office and factory areas were designed in an efficient fashion. ADT's decision to experiment with the inherited still-born factory also serves to illustrate the Dutch view of space. The 'deadline' episode at ADT is a classic illustration of how the Dutch view time, and how that view can cross with other meaning of time, as will be duly elaborated.

The Thai, by contrast, take environment as a gift, to be thankful for and revered (Klausner 1993, Komin 1991). Natural environment is deemed sacred and animate; there are gods for all various natural artefacts - rivers, mountains, valleys, forests, trees, rain, drought, the sun, moon, star, sky, and so on. The famous annual festival 'Loy Krathong,' for example, has its origin on the belief that the rivers are animated entities, personified by the mother of waters or 'Mae Khongkha'. The ritual illustrates in many subtle ways how the Thai have woven their view of natural environment into the fabric of their lives. During the festival, on the full moon of the twelfth lunar month, when the rivers are in high tide, the Thai will go out in the evening with decorative floats ('Krathong') made of leaves and flowers and adorned with candles and incenses, that they would let adrift on the waterways. This act signifies several beliefs 
simultaneously. First and foremost, it is a gesture of gratitude as well as remorse for what the waters have given to their lives and what harms they may have incurred to the waters through their use. The ritual has also developed as a way for many Thais to mark a sort of new beginning, asking blessings from the mother of waters, for a new lease on life in the year to come. Countless other rituals are associated with how the Thai look at natural environments not as physical objects but animated entities. Even in modern days, many Thais, when visiting or settling down in a new place, would ask for permission and blessing from the 'mother or father or spirit' who owns that place.

Abstract environment is regarded by the Thai in a similar fashion. Time is regarded as part of life, it is a mere point of reference and its meaning is relative. As Klausner put it, 'the Thai are averse to being slaves to the clock.' (Klausner 1993: 334). Time, in other words, is part of what transpires, and not a dictator of it. In fact, the Thai tend to regard haste as something that could lead to undesirable results, such as mistakes and inefficiency. When the Thai make an appointment, the time is set as just a convenient reference point, not a precise contract. Arriving early or late for an appointment is thus not a serious crime, an issue that very much frustrated many of the Dutch characters in the cases. No wonder, at ADT, deadline for the Thai employees was neither definite nor critical; it was mainly a point of reference. More, since time is part of life, past, present and future are regarded as a circle, rather than a linear progression, a concept that is rooted in Buddhist view of life as one continuous series of karma (Klausner 1993), and hence quite difficult to explain to Westerner, who hold a linear view of time. This may explain partly how Hofstede's long-term orientation index put Thailand in the top ten, ranked 8 with a score of 56 . However, for the Thai, it is not exactly that they are long-term oriented, but rather that for them, past, present and future are very much related, simply because they are all part of life. As for space, the Thai regarded this as part of nature and so their view of space is similar to how they view natural environment. The Thai concept of space is very similar to the Chinese concept of 'feng shui' as will be explored further in the upcoming narration of the CDW case.

\section{The meaning of '(inter)relationship'}

Naturally, the meaning of (inter)relationship in the Dutch and the Thai cultures follow fairly straightforwardly from how they view self, others and environment.

Put simply, for the Dutch (inter)relationship between and among those three basic entities are based primarily on the relative contributions of each of those entities to a situation. The Thai, however, define (inter)relationship first and foremost based on the relative roles of those entities and how the roles are to be fulfilled. Since relationship and interrelationship are diverse and very much situational, the discussion on this issue is kept at a broad description for now. Subsequent discussions, however, explore a few more specific cases of (inter)relationship further as their relevance emerges. 
W ith this image of culture as intricate webs of meanings, we can now Venture into the virtual world of another set of intricate cultural webs - the case of CDW, an odd-eyed cat.

\section{. 333सम8E8.}





\section{Chapter 5}

\section{Case CDW}

\section{Portrait of an odd-eyed cat}

I think the "how-to" courses and trainings are useful but I cannot say that I've relied on them much myself. I picked up on things by myself. Maybe I don't do it right either. Who knows?

Karel Westerveld President, CDW

2006

\section{. 3334\$8E.}

So, for me, I find that I have to be very flexible in my style. I have to adapt my style to all these different styles of various cultures. I dance to their steps, to speak metaphorically, just so we can dance together.

Choawalit Saenamuang Vice President, CDW

2006

\section{. 333\&>8E.}

I mean, to tell you the truth, I still have a very hard time to get acquainted to the Netherlands. I mean I'm Dutch. Dutch is my mother language, but Dutch people are holding off, eh? Very difficult to get to be friend with Dutch people. 


\section{Reflection 10: I am captivated by cats and addicted to quotations}

THE central metaphor that came to my mind for the case of CDW certainly had something to do with my fascination with cats. I wonder if other researchers would think of something else to depict the key theme in this narrative.

I am also addicted to quotations, mostly through reading - a habit I developed during my days of literature study. When you study literature, you need to make notes of quotations so that you can use them to support your argument and analysis of the work. From this practice, I developed a habit of 'bookmarking' anything I find interesting in my reading. This way, I can quickly go back to them. I find myself doing this even when I'm reading or listening to non-academic work such as advertising, package labelling or music. Over the years, I have accumulated a good stock of favourite quotations.

Surprisingly, in more than a few occasions during the course of this study. I suddenly 'understood' what I was struggling with in my conceptualization by relating the issue to a favourite quotation. Some were from my literary collections and favourite lyrics, and quite a few were passages in detective books that were my usual bed-time or travelling companions. A few of these found their ways to be the preluding quotations that serve as a lead-in to each non-case chapter and also at the beginning of this manuscript.

Thus, these 'preluding' quotations actually aided me well as an analysis and articulation tool. They 'marked' what I deemed to be the key messages for the entire book and each individual chapter. I didn't at first intend them to actually be included in the final report. I simly put them there in earlier drafts as a sort of reminder or signpost to myself. That was how I related to these lead-in quotations during the writing and analysis process. I planned to take them out in the end as they didn't seem apt for academic work. But then, how can I not acknowledge their indispensible service? In the end, they remain a part of this final manuscript, so unconventional and non-academic as they may be.

Now, back to the cats. My choice of metaphor for the case reveals another personal inclination that certainly influenced my interpretation and delivery of this study. I relate what and whom I investigate to something I love. I am a true Thai when it comes to the two issues of face and gratitude. Readers may already detect my rather obvious 'nice' view of the actors in the first narrative of ADT. I'm afraid this will also be true of the subsequent ones. In a sense, I am a living victim of the Thai smile. Besides having difficulty in saying anything bad about my informants, because I do not want to slight either their or my faces, I also feel forever grateful to their generous help. As a Thai, it is a sin to think badly about anybody who has been kind to you. Thus, I must plead guilty to being partial to my informants. Yet, this realization eventually served as an effective self-check mechanism throughout my analysis - whenever I found my interpretation to be too positively partial, I double or triple checked my theorization and many times this conscious act indeed helped me see the issues more clearly and improve the end results.

I have never seen a real-life odd-eyed cat, let alone being granted the honour of having one as a companion. Yet, maybe I can claim CDW to be a virtual odd-eyed cat I have had the privilege to come to know. 
Diagram 5.1: $\quad$ CDW fact sheet (As of 2007)

\begin{tabular}{|c|c|}
\hline Industry & civil engineering/consultancy \\
\hline Product/service portfolio & $\begin{array}{l}\text { civil engineering consultancy, primarily in architecture } \& \\
\text { construction and presently also water \& environmental }\end{array}$ \\
\hline Market portfolio & business firms; governmental and other agencies \\
\hline Core competency & $\begin{array}{l}\text { design, engineering, architecture, and project manage- } \\
\text { ment }\end{array}$ \\
\hline Operating portfolio & $\begin{array}{l}\text { complex operating structure that encompasses all as- } \\
\text { pects of civil engineering project - i.e. conception, de- } \\
\text { sign, construction, and project management, primarily } \\
\text { of large-scale construction }\end{array}$ \\
\hline Relative size $^{1)}$ & $\begin{array}{l}\text { large, being a partially-owned subsidiary of a large } \\
\text { Dutch engineering consultancy multinational (RHG) }\end{array}$ \\
\hline Headquarters & $\begin{array}{l}\text { corporate headquarters (RHG): the Netherlands; } \\
\text { Asian headquarters (CDW): Bangkok, Thailand }\end{array}$ \\
\hline Divisional offices & $\begin{array}{l}\text { global: complex network of branch offices, subsidiaries } \\
\text { and partners worldwide; } \\
\text { in Asia: local offices in Cambodia, Vietnam and the Phil- } \\
\text { ippines; and representative/partner offices in China, } \\
\text { India, Indonesia, and Malaysia }\end{array}$ \\
\hline Corporate culture & $\begin{array}{l}\text { global: fairly structured, with a complex structural and } \\
\text { hierarchical arrangement } \\
\text { in Asia: engineers and architects were the heart and } \\
\text { soul of the company and their professional cultures } \\
\text { dominated corporate culture }\end{array}$ \\
\hline Workforce $^{2)}$ & $\begin{array}{l}\text { global: } 4000+ \\
\text { CDW total in Asia: } 400+\text { and CDW Thailand: ca. } 250 \\
\text { [the Thai office: } 1 \text { expatriate MD; } 5 \text { expatriates, } 1 \text { local- } \\
\text { hired Dutch; } 10 \text { Thai management staffs; } 50 \text { Thai profes- } \\
\text { sional staffs; } 100+\text { Thai supporting staffs] }\end{array}$ \\
\hline Manner of entry into Thailand & semi-accidental, following a major consultancy project \\
\hline Operating portfolio in Thailand & comprehensive civil engineering consultancy \\
\hline $\begin{array}{l}\text { Years in business } \\
\text { Years in Thailand }\end{array}$ & $\begin{array}{l}125 \text { (RHG) } \\
32(\mathrm{CDW})\end{array}$ \\
\hline
\end{tabular}

Note: 1) defined very loosely as small, medium, and large, based on rough benchmark vis-à-vis overall global operation of the entire corporation relative to average multinationals.

2) as of 2006 . 
ITTING NOW IN A QUIET, COSY LIVING ROOM OF HIS APARTMENT IN LEIDEN, THE NETHERLANDS, Khun Geert, Co-founder and former President of CDW, often reminisced about the many memorable years he lived and worked in Thailand. He was back 'home' now, in a sense, since this country was where he was born and raised. Yet, in a way, here was no longer entirely his home, well, not his 'only' home at the very least.

Thinking of his time in Thailand, he could not help musing about how his enterprise there evolved from a business partnership into a life-long friendship with one 'odd' character, Khun Chatoeran - the only Thai who ever uttered 'now, damn it' in his face. Together, they built a successful joint-venture, and out of that, their partnership had gradually turned into a life-long friendship that Khun Geert still held dear. Come to think of that, Khun Geert thought it would be nice to brew that tea from Khun Chatoeran's retirement venture of organic farming. As he sipped the tea, Khun Geert thought, the tea was not bad, not bad at all. As a matter of fact, it might be of a quality just as rare and fine as that of the person who cultivated it.

At the same moment, people back at CDW office in Bangkok carried on the legacy of the two 'parents' of CDW. The 'offspring' of this partnership, a successful Dutch-Thai joint-venture, turned out to be a rather fascinating creature - an odd-eyed cat of sort.

Let us see what we could make of this intriguing creature.

\subsection{Meet the odd-eyed alliance}

CDW was a Dutch-Thai joint-venture consultancy providing services in civil engineering field with extensive expertise in architecture and building and prospective expansion into water and environment. CDW's portfolio of expertise included: feasibility study, design, engineering, interior work, construction management, and project management. CDW's activities span the range from conception, construction and maintenance of industrial and commercial facilities, office buildings, civil works, and infrastructure, with recent addition of maritime works and water and environment. As of 2007, CDW had over 30 years of experience in Thailand and the South-East Asian region. The firm had become a partially-owned subsidiary of a large Dutch engineering consultancy, a status which reaffirmed as well as enhanced its solid position in the Thai and Asian market.

\section{The big picture}

To be precise, CDW became a part of RHG, a top-notch large Dutch engineering consultancy, after RHG acquired CDW's former Dutch parent company, DWG, in the early 2000s. RHG was a century-old, prominent Dutch engineering consultancy that operated a full range of civil engineering activities with the following divisions in its operating portfolio: spatial development; infrastructure \& transport; building \& architecture; building management \& consultancy; building services; industrial installations; water; environment; coastal \& rivers; and maritime. RHG was active in Europe, Africa, Asia, the Middle-East, and Latin America with a vast network of 11 operating divisions and 
numerous operating offices in over 26 countries spanning five continents. Globally, RHG had well over 4,000 employees and an annual turnover of $€$ million 327 in 2007.

In 2001, as part of RHG's expansion plan into Asia, the firm acquired DWG, which was the 'Dutch' parent company of CDW, thereby inheriting the joint-venture CDW. In fact, CDW was an integral part of the attraction for the acquisition of DWG, because RHG saw great potential in this burgeoning Asian offspring of DWG. CDW had operated as a lucrative regional arm of DWG and hence could serve as an effective channel for RHG's move into Asia. That such a prestigious firm as RHG considered CDW a prospective Asian addition to its vast global network served an impressive testament to the success of CDW.

However, to fully appreciate the current outlook of CDW, a long and complex history of its conception and advancement is in order.

\section{The origin}

In the early 1970s, a burgeoning Dutch civil engineering consultancy - DWG, won a contract to design and manage a major construction project of a new head office building for a prominent Thai governmental agency. This was of a landmark calibre and an impressive achievement of DWG representative: Dhr. Geert Halsema, especially since DWG did not even have an office in Thailand at that time and was working only from its site in Indonesia. The nature of the civil engineering industry, however, necessitated a need to incorporate local presence and knowledge to meet legal requirements as well as to attain familiarity with domestic practices. So, the Dutch firm sought and found a local partner in a young and dynamic Thai architectural firm - D321, owned and managed by a budding Thai architect, Khun Chatoeran Prempreecha.

The initial intention of the alliance was only to carry out the one particular prized project. However, the union turned out to be quite a success, not only with regard to the project itself, but also, and perhaps more impressively, in relation to the resulting synergy of the two partners. Due to this success, the two companies decided to join together and found CDW as a joint-venture. CDW was in effect a hybrid offspring of DWG and D321. Both parents of CDW maintained their presence and continued their businesses as before - DWG as an international Dutch engineering consultancy and D321 as a Thai architectural firm - while the offspring firm CDW took on the task of exploring a lucrative niche market, targeting multinational companies that were expanding their operations into Thailand and in need of architectural and construction expertise to set up their facilities.

\section{The evolution}

The two decades after the birth of CDW proved to be a prosperous period for the new firm to exploit. The Thai economy was blooming into a little tiger, with an influx of huge multinationals, all wanting to establish firm footing in Thailand and the neighbouring countries, starting with proper offices or plant facilities, or both. CDW, backed-up by the image of its Dutch parent, could attract an impressive number of 
high-calibre projects from well-known multinationals, particularly those from the Netherlands and Western Europe. At the same time, with support from D321, CDW also rapidly secured a stronghold among prestigious Thai clients, governmental, nongovernmental, and business alike. CDW's list of past, on-going and repeated clients read like a roll-call of prominent businesses and agencies, including such names as: Akzo Nobel, Bank of Thailand, Bayer, Carrefour, Coca Cola, Charoen Pokphand (CP Group), Dow Chemicals, Foremost Friesland, Heineken, Hoechst, IBM, Johnson \& Johnson, Kellog's, Kodak, KLM Royal Dutch Airlines, Lotus, Makro, Merck, the Netherlands Embassy, Nestlé, Orange, Philips, Q8, Royal Dutch Shell, Seagate, Siamcona, Thai Baroda, Thainox, Thompson, Tops, Unilever, Unithai, and Wella.

CDW grew impressively over the years, expanding its operations into other countries in the South-East Asian region and accumulating a remarkable portfolio of projects. CDW Thailand was eventually established as the regional hub of the CDW group even though the Indonesian office of DWG was its predecessor in the region for the Dutch parent. This was partly due to the advantageous economic and political environment in Thailand at that period and partly a result of the effectiveness of CDW Thailand itself. As of 2007, CDW had branch offices in Cambodia, Vietnam and the Philippines, plus representative or partner offices in China, India, Indonesia, and Malaysia.

Externally, CDW operated independently from its parent companies but exhibited a strong tie with both. It was virtually the operating arm of DWG for the Dutch firm's Asian operations while close partnership with D321 continued until these days. Internally, the three companies were virtually one close-knit family. For all practical purposes, the three firms operated very much as one party, drawing resources from one another and capitalizing on the diversity of strength within the group.

\section{Present day CDW}

Hence, when RHG acquired DWG in 2001 and CDW became a partially-owned subsidiary of RHG, CDW continued to operate independently and under its own name, due to the goodwill of its reputation in Asia. It appeared likely that CDW would be gradually absorbed under the umbrella of RHG while still maintaining its high operating autonomy and identity. RHG had no immediate plan to completely assimilate CDW into its constitution. The plan was, on the contrary, to continue capitalizing on CDW's reputable goodwill in the Asian market by letting the joint-venture operate independently in its captured market of building \& architecture while gradually injecting whatever extra capabilities RHG had to offer into the local operation in Asia. For instance, RHG set up a branch office under its own name (RHG Asia) to explore opportunity in water \& environment sub-sector, where RHG had already established a solid status worldwide. To the outsider, however, CDW would still be the same reputable consultancy, only with even more 'promise' that was strengthened by the calibre of its new esteemed Dutch parent. 
As of 2007, the entire CDW/RHG group of offices in Asia employed a total of 400 plus workforce, with Bangkok as the home base. CDW office in Bangkok (inclusive of D321 and the newly registered RHG Asia) had about 250 employees, most of whom were local Thai staffs in the middle management, professional (including engineers, architects, draftsmen, and construction supervisors) and supporting functions. The need for expatriates was only for high-level and special positions that required the expertise, seniority, or calibre of expatriates. The top position of President had always been held by Dutch expatriates. Generally, CDW had about 5 expatriate posts, all of which were in the high-ranked professional and top management functions.

\section{The Actors}

\section{Co-founder and first President: Dhr. Geert Halsema (Khun Geert)}

Dhr. Halsema was the Dutch co-founder and first President of CDW. Although already retired from active function, he continued to serve as Executive Consultant on an advisory basis. This reflected both the wealth of experience that he had accumulated and the deep affinity he had developed for the firm, during the almost three decades that he managed it. Dhr. Halsema and his Thai counterpart, Khun Chatoeran, were the key figures in the DWG-CDW-D321's birth, growth and success in Thailand and the Asian region.

The major part of Dhr. Halsema's career was as an expatriate executive for DWG. He travelled extensively and had exposure to many foreign cultures. Prior to taking up the position of President at CDW, he managed DWG office in Jakarta for a short period. After that, he spent the next three decades building and managing CDW until his retirement around the later part of the 1990s.

After retirement, Dhr. Halsema and his wife decided to move back to the Netherlands to be closer to their children and grandchildren. However, beside Dhr. Halsema's advisory role at CDW, he and his wife also maintained a strong personal tie with Thailand and still visited the country and many friends there on a regular basis. Already retired, Dhr. Halsema's concern with regard to CDW was mainly that the legacy that had made CDW what it was at present, and of which he was a key part, would continue to flourish and fuel CDW business further into the future.

Dhr. Halsema is referred to as Khun Geert in the narrative.

Reflection 11: Khun Geert - a Dutch 'gentle' man!

My first and lasting impression of Khun Geert was that he was such a kind but elegant man. He reminded me a lot of my own father-in-law (also a Dutch from the same generation as Khun Geert). They were both wise, professional, experienced men whose characters managed to demand respect as well as induce comfort. Throughout the interview and casual talk with Khun Geert, I had this feeling that he was very willing to share his experience with me, less because he wanted to talk about it, but more because he 'felt' it would be useful for me. Yet, there was nothing arrogant about his delivery. He was not 
showing off; he was simply passing on the story. I believe this level of stature can only be manifested in an individual who has amassed more than his due share of contributions and can afford to be so comfortable with his own identity that total raw honesty is the only manner of expression.

This impression certainly affected the way I received and interpreted the meaning of his message. My only defence is that it motivated me to be just as honest about my examination of the message as Khun Geert was in his delivery of it.

\section{Co-founder: Khun Chatoeran Prempreecha (Khun Chatoeran)}

Khun Chatoeran was Khun Geert's Thai counterpart in the history of CDW. Khun Chatoeran was of a privileged rank in the Thai society when he started his architectural consulting firm D321 around the end of the 1960s. Western-trained, highly qualified and extraordinary progressive, he welcomed and stood on par with his Dutch counterpart, a key to the success of the joint-venture. While Khun Geert took on the position of President of CDW, Khun Chatoeran kept his position as D321 Director. With Khun Chatoeran at the helm and acting as the needed linkage, D321 provided Thai fluency and architectural capability for CDW's projects. More, Khun Chatoeran made sure the Thai firm's identity helped provide a trustworthy Thai front to attract prestigious local clients. The result was that after three decades, D321 was highly regarded within the Thai architectural community.

Although trained professionally as an architect, Khun Chatoeran had high management competence and keen business sense. Thus, even though he had officially retired from the firm, he still exercised an active advisory role, just like his Dutch counterpart. Progressive all through his life, he spent most of his time after retirement in an up-country farm, cultivating organic tea - a hobby that he thought may turn into a good little business later on.

\section{Reflection 12: Khun Chatoeran - a Thai 'gentle' man?}

Due to resource constraint, I did not have a chance to meet Khun Chatoeran in person one of the few things I regretted in the course of this study. But such is a constraint in any study. So, I had to limit his (indispensable) portrait to the remarks from a few thirdperson impressions. I found this to be an intriguing exercise. From the little that I knew of him, I had to piece together a sketch of a man that was as true to life as possible.

I conjured up Khun Chatoeran's character from a few signs in the tales. My first-hand knowledge of how Thailand was in the 1970's helped me realize that Khun Chatoeran had to be at the forefront of his generation. Few Thais in those days would have the Western training and progressive outlook that he seemed to exude. Even fewer would have the means and fortitude to set up businesses of their own. In those days, he must have been among the few contributing elites, literally as well as figuratively, who helped to move the Thai business and economic landscape to where it is now. Even in his retirement, I believe he still commanded the same quality and stature. It would be hard-pressed to find a Thai 
of his generation who is passionate about farming organic tea, let alone willing and able to materialize that passion.

I believe he is someone I would very much enjoy meeting face to face.

\section{President: Dhr. Karel Westerveld (Khun Karel)}

Dhr. Karel Westerveld was a Dutch in his fifties. He originally worked for DWG at its location in the Netherlands. Then, during the 1990's he was involved in several projects in Asia, including India, Indonesia and Thailand. After a period of extensive travelling and involvement in the Asian side of the business, Dhr. Westerveld decided that he would want to move and work in this part of the world on a more permanent basis. Although the corporate bosses were not quite enthusiastic with Dhr. Westerveld's request, they eventually agreed to his taking up a top position at CDW Thailand.

After the retirement of the first President, Dhr. Westerveld took on the position of CDW President. Shortly after that came the acquisition of DWG by RHG. Dhr. Westerveld continued a dual role of President for both CDW and the newly established RHG (Asia) during the interim period before turning over the position of CDW President to the third and current President, also a Dutch expatriate. As President of RHG (Asia), Dhr. Westerveld was the top most executive of the RHG operation in Asia. Although this position meant that he would be more involved with a broader mission of RHG (Asia), Dhr. Westerveld continued lending guidance and supervision to CDW activities, as CDW remained the most significant part of the entire Asian operation, at least during the immediate period after the acquisition.

Dhr. Westerveld is referred to as Khun Karel in the narrative.

\section{Reflection 13: Khun Karel - a CEO and a professor}

My first impression of Khun Karel was that he seemed a very reserved Dutch and a typical high level executive. Prior to the actual interview, I only communicated with Khun Karel via his secretary, Khun Busara, and somehow I was quite nervous before my meeting with him. When I actually met him, I still felt a bit anxious, especially because of the formal setting of his office. As the interview went on and I learned more about him, however, I suddenly felt as if I were in the Netherlands. Khun Karel reminded me of quite a few Dutch that I have learned to get used to, after overcoming an initial apprehension of their reserved manner. I gradually realized that although Khun Karel was reserved, he was neither unkind nor inhospitable.

I found it rather surprising, though, that Khun Karel liked to live and work in Thailand, because he seemed still quite Dutch. He also showed understanding of the Thai culture without losing touch of his Dutch heritage. To me, he stood as an amiable UnDutchables, one who retained his original cultural profile but also sincerely appreciated those of others. By the end of this project, I learned that Khun Karel eventually left CDW to take up a position as a Professor at a Dutch university. Somehow this didn't surprise me at all. 


\section{Executive secretary: Khun Busara Navara (Khun Busara)}

Khun Busara was an active lady in her mid-thirties. She came to CDW as a young secretarial intern. Her performance and rapport with Khun Geert, however, secured her a permanent position with the firm, moving up from a junior to the most senior secretarial position as Executive Secretary to Khun Geert, for over fifteen years. Then, she continued on in her function assisting Khun Karel and the third President. Khun Busara was thus one of those loyal CDW old-timers.

As is the case with many secretaries, particularly executive secretaries and especially in Thailand, Khun Busara was virtually the gate-keeper and semi-butler for the top boss. As Khun Geert put it fondly, she, in her capacity as executive secretary to CDW President, 'was the most important lady in the firm'. Khun Busara was the one to make sure that whatever demanded the boss' attention were channelled through the gate and whatever might waste his time and energy got sifted out. This meant that she needed to be fully and constantly aware of the President's businesses and priorities. Not only that, in a true Thai tradition, Khun Busara also took care of certain personal matters for her bosses. As a Thai, she could handle several matters for the expatriates more efficiently and it was not uncommon for her to be running personal errands for the top bosses, leaving them time to deal with business matters.

Khun Busara's role was thus a blend of interpreter, evaluator, coordinator, bridge and sentinel. This applied to all parties she dealt with, outside as well as inside the firm. She was, in many ways, the hub of what was going on at CDW, internally and externally, for all things great and small, or 'from toothpick to submarine' as the Thai expression goes. Her role indeed went further and beyond official secretarial duties as many Westerners may perceive. It would not be an exaggeration to say that if one wanted to know what's going on at CDW, one only needed to ask Khun Busara. Khun Busara's main priority was to function as a gatekeeper and coordinator for her bosses while at the same time served as an invisible 'link' between the various hierarchical and functional slices of the firms.

Reflection 14: Khun Busara - the gatekeeper

Khun Busara's stern voice over the phone in her very first phone conversation with me puzzled me a great deal, especially in contrast to her very pleasant and cordial second phone call with me, in which she confirmed my appointment with Khun Karel. I only found a clue to this puzzle after I had a chance to meet her in person and appreciated her role as the gatekeeper at CDW. My first call to her caught her by surprise and she needed to 'guard' her boss from a 'strange' request. Her call-back to me was after she had learned more about me and her boss' position regarding my interview request. Once I realized this, I could see how earnestly Khun Busara carried out her responsibilities and how crucial her earnestness must be to her boss.

Then, when I had a chance to interview Khun Busara, I got to like her quite a lot. She was sincere and eager to share her experience. She was very humble at first, not realiz- 
ing how her 'secretarial view' could be of use to an academic study. But once I assured her, she was very quick to comprehend the meaning of the interview and opened up herself. I appreciated her earnest and vivid stories, which provided me with yet another unique set of evidence, one I can only get from a person in such a role. Khun Busara was the only 'secretary' I had a chance to talk to for this study, and her contribution to my analysis was thus most valuable. Looking back, I believe it would have been a good idea to seek more informants of similar profile as that of Khun Busara, not only for the constant comparison and triangulation purposes, but also for the refreshingly different take on the issues.

\section{Vice President: Khun Chaowalit Saenamuang (Khun Chaowalit)}

Khun, or rather Dr. Chaowalit Saenamuang was one of the top 5-7 project managers at CDW. By training, he was a highly qualified engineer, with three Master degrees from well-known Thai and German universities (two in engineering and one in business administration) and a doctorate in operation management from a respectable American university. In many respects, Khun Chaowalit carried on the legacy of Khun Chatoeran, that of a progressive, Western trained, elite Thai. Just like Khun Chatoeran, Khun Chaowalit understood the concerns and needs of his Thai colleagues and clients but also empathized with Dutch/Western sentiments and attitudes. He appreciated both, as a matter of fact, and hence played a vital role in the continuation of the legacy of the Thai co-founder. Khun Chaowalit professed substantial affinity for the Dutch in particular. He found them to be pleasant to work with, both as clients and colleagues. He liked the no-nonsense, direct style that was typical of the Dutch he met and the low-key sense of humour they expressed at social occasions.

Khun Chaowalit worked mostly as a project manager and hence was at the centre of diverse activities, functions, professions, and interests. His most current position was that of a Vice President in Business Development, targeting a specific segment of the market, namely logistics and operations management projects for large retailers. He was involved with the entire spectrum of a project, from bidding to completion. As such, he must deal constantly with colleagues from all internal departments at CDW, as well as with outside authorities, contractors, suppliers and the clients themselves. In a nutshell, he was a conductor of a large symphony orchestra that included a variety of professions and agendas. His main concern was, like that of any maestro, to achieve harmony while bringing out the best in each and every member of the ensemble. $A$ successful bidding and completion of a project would stand as the ultimate testament to how well Khun Chaowalit performed his function.

\section{Reflection 15: Khun Chaowalit - the conductor}

To me, Khun Chaowalit was one of those rare individuals: 'practical intellectuals'. He was intelligent as well as smart. His academic achievements were a testament to his intellect. His smartness, however, only revealed itself in the manner and content of what he shared 
with me during the interview. I learned much from the many stories that he told with lots of humour - how he observed the subtle differences among the variety of supposedly similar cultures such as among the diverse Westerners (Dutch, German, French, American) or among the many Easterners (Hong Kong, Singaporean, Taiwanese, Thai); how he viewed the tension between the local and expatriate within his own firm; and what it took to carry out his function. His down-to-earth attitude balanced well with his professional qualification and I had no doubt he used them both to great advantage in his function as a project manager. I have to admit that much of the analysis of CDW was influenced by my adopting a viewpoint very similar to that of Khun Chaowalit's.

\section{Supporting cast}

Beside Khun Karel, there were about 4-5 other Dutch expatriates at CDW, all of whom were in high ranked professional or managerial functions. These expatriates interacted primarily with high-ranked Thai personnel within the firm (such as Department Heads and Project Managers) and their counterparts in the clients' organizations. Middle and lower ranked Thai personnel did not usually have direct or frequent contact with expatriates, although they did pay attention to the influences and activities of the expatriates. A single unique foreigner in the firm was a local-hired Dutch who had adopted Thailand as his new home and was contented to be employed as a non-expatriate Dutch.

\subsection{It takes two to tango}

CDW had a robust tradition of the strong Thai-Dutch partnership and took pride in this. The company's name itself reflected a conscious effort to nurture a mutual respect in this partnership. The Thai part of the name used the first name of the Thai co-founder rather than his family name, following a Thai convention, while one of the words in the company name used Dutch spelling for a similar English word to reflect the Dutch legacy. This conscious blend of the two heritages was as much a desire as it was a necessity - the unique blend of international/Dutch aura and local/Thai fluency gave CDW an edge in its target market.

In the civil engineering consultancy business, what a firm virtually sells is a 'promise' - that is, a proposal of a product yet to be delivered. As such, CDW operated in an environment where prestige and reputation were the top-most critical success factors. CDW's clients were large organizations - commercial corporations as well as public agencies. Its products were in essence engineering and architectural capabilities, which can be visibly manifested only in successfully completed projects, in the form of landmark office buildings, prolific production plants, or celebrated public facilities and infrastructures. To win high calibre contracts that involved hefty outlay on the clients' part, CDW needed to constantly maintain and build on the 'image' of its capabilities, by capitalizing on past successes as well as cultivating new accomplishments. This required a harmonious interplay between 'perceived' and 'real' qualifications: that is, the joint-venture had to be able to make a convincing impression to win a contract, and 
then delivered a completed project that at least met or, better yet, exceeded that impression. More, since CDW targeted huge projects of high calibre and price-tag, from clients that operated at the global playing field, the firm needed to strike a good synergy between global expertise and local fluency. As such, promises and achievements (or perceived and real capabilities) had to make up two side of the same coin for CDW.

In the same vein, global prestige and local stature also had to reinforce each other and made up two sides of yet another coin for CDW. This translated CDW's daily operation into a complex orchestration of myriad capabilities. Its core competency resided in those of its key professionals: the chief engineers and architects, whose professionalism and qualification marked the beginning and conclusion of a project. At the same time, the supporting teams of project management, construction \& interior work, and quality control must operate to carry out their functions effectively to ensure that each project was delivered as conceived, planned and pledged. In addition, CDW also needed to cooperate well with external parties such as outside contractors and governmental agencies. Finally, one of CDW's key strategies was to maintain a constant partnership with clients throughout a project life-cycle to guarantee clients' satisfaction as well as to pre-empt costly rework.

Partnership was thus the origin and lifeblood of CDW - partnership of myriad actors, activities, functions, professions, entities, agendas, interests, or in a nutshell, partnership of cultures at many levels and in many ways, shapes and forms.

This here is a tale of a tangle of myriad cultural partnerships that eventually bred an intriguing odd-eyed alliance.

\section{An ideal match: a tale of two founders}

To Khun Geert's mild surprise, he found himself having to 'grow' back to the Dutch culture once he and his wife settled down in the Netherlands again after three decades of expatriate life. At the shop the other day, for instance, he found himself feeling affronted when addressed by a young assistant with the familiar 'je' instead of the respectful 'u.' ${ }^{21}$ This would never happen in Thailand. No young Thai would behave that way to a man of his own maturity and stature. His wife was at this very moment studiously studying for an entry exam to be a member of a golf club. In Thailand, she was as good as 'cordially invited' to take up membership in an equivalent Thai establishment. So, they both now had to try to grow back into the Dutch way of life that had faded into the background over the years.

This was nothing new to Khun Geert, of course. He had done it many times before, this 'growing into' an unfamiliar way of living. As a lifelong expatriate, Khun Geert learned earlier on that one had to gradually adapt to a new culture if one were to survive and thrive in it. Of his years in Thailand, he was glad that the adapting experience

\footnotetext{
${ }^{21}$ In Dutch, two forms of second person pronoun exist: the formal ' $u$ ' and the intimate 'je.' Interestingly, the Thai language has a similar, although much more elaborated, system of distinction in the proper use of all pronouns (a subject that deserves an entire chapter in itself).
} 
turned out to be memorable in a pleasant way. For that, he felt forever thankful for a partnership he had with his Thai counterpart, Khun Chatoeran. As a matter of fact, Khun Chatoeran has eventually become not only a fine business partner but also his lifetime friend.

Their partnership was to Khun Geert a true partnership, an ideal match, if there was such a one. Khun Geert considered himself lucky to have Khun Chatoeran as his counterpart in the needed Dutch-Thai alliance when he first entered Thailand. They complemented each other well and operated on equal footing. He now remembered fondly the usual shouting matches they had had over the years and how grateful he was that Khun Chatoeran would be so comfortable with direct confrontation, without ever making a personal fuss about it afterwards like most Thais would have. The usual shouting matches would transpire; frictions got sorted out; and their partnership and friendship kept on without a dent, so to say. Things would not have been so smooth and productive if Khun Geert had to struggle with the usual round-about Thai way in his dealing with a Thai partner when the business was still in its embryonic stage.

Indeed, many a time Khun Geert wished that he could find this raw honesty in other Thai colleagues and personnel. He still remembered with a twinge of astonishment how a trusted staff would come up to him one day out of the blue to hand in his sudden resignation. There were quite a few of such occasions to sadly puzzle and upset Khun Geert. A few of them were even those he had set his eyes on to succeed him when he would retire. Indeed, it was because of the repeated cases of sudden resignation of three 'heirs apparent' that in the end his successor eventually had to be a Dutch.

These sudden-departure cases were Thai employees who usually just preferred to say goodbye right then and there without any pre- or post-amble whatsoever. What he found most astonishing was that these were usually the very people he had never imagined to be ones who wanted to leave the firm. They seemed so happy and fitted in. They never gave a hint of any dissatisfaction or desire to leave, but when they did, they only wanted to just turn their back and walk away without any explanation. Khun Geert would not have been so bewildered if the departure was not so sudden and the goodbye so abrupt. But that seemed to be the preferred fashion for many Thais. Why couldn't they just give it to him directly but smoothly? That would have been easier to swallow than this mysterious swift cordial goodbye. After all these years, this remained the one frustrating memory of his time in Thailand. The memory reminded him of the one thing he liked about Khun Chatoeran, though - the upfront, say-it-as-it-is style that gave mystery not a chance and left the air clear and easy to breath.

So, they learned to 'use' each other, to exploit each other's complementary quality for the benefit of a mutual business advantage. Khun Geert focused his effort on developing CDW for the then parent DWG and he certainly needed the rare, yet ideal, blend of quality he found in Khun Chatoeran: local familiarity, Western sympathy, and honest - Dutch-like - style of direct interaction. Khun Chatoeran, as a Thai who appreciated the value of Western know-how for a still developing economy of Thailand, wel- 
comed the advanced technical expertise that he could tap on through the Dutch-Thai partnership. More, he could even use the Dutch blunt approach, in the living figure of Khun Geert, to achieve certain results with his own countrymen when he knew he himself could never do so as a Thai. When the "kreng jai' ${ }^{22}$ value could get in the way of business, he would turn to Khun Geert to cut through it, since the Thai would not take to the direct approach that seriously when it came from a 'farang' ${ }^{\text {'3 }}$. Khun Geert, on the other hand, relied on Khun Chatoeran to openly and honestly share with him local fluency that he needed to run the business successfully. Thus, although heading two seemingly different companies - Khun Geert as the President of CDW and Khun Chatoeran as the Director of D321, the two co-founders of CDW operated virtually as one team, one family.

Their synergic partnership indeed fuelled the eventual growth of all the business entities they founded and managed. It also established the identity of the sequel of their initial alliance, in more ways than one. Even when both the co-founders have retired, the legacy of the intriguing partnership still permeated the present CDW-D321 group.

As for the origin of that legacy, the ties still bound. Both Khun Geert and Khun Chatoeran maintained advisory roles in the CDW-D321 group, and their experiences were often valuable to the current generation. Personally, they remained close friends and enjoy each other's company on a regular basis. Their children had also become good friends. Khun Geert kept his apartment in Bangkok where he and his wife stayed during their regular visits to Thailand, something that served as a routine get-away, especially whenever the Dutch weather got a little too cold for comfort. Khun Chatoeran lived now in the North of Thailand where he enjoyed working on his farm, cultivating organic tea, a passion that might develop into a fine little hobby-cum-business one day.

\section{A curious offspring: an odd-eyed cat?}

With that distinctive origin, CDW became a product of a unique union and itself appeared a rather curious fusion. If CDW were to be a biological entity, its appearance would probably carry a telltale sign of its crossbreed. CDW might then be akin to those peculiar creatures - the odd-eyed cats (each eye being a different colour, such as one blue eye and one orange or copper eye). This is a striking but apt analogy, for CDW thrived on an odd form of various partnerships. By odd is meant that all the partnerships at CDW were based on one crucial characteristic - the need for each partner to uphold its own unique quality while contending with and exploiting the unique quality of the other partner(s) at the same time. In such a particular form of partnership, if one partner were to be completely absorbed into the other's identity, the partnership

\footnotetext{
22 'Kreng jai' is earlier explained in Chapter 4 of this manuscript.

23 'Farang' is a Thai word for Westerner, most likely originating from the English word 'foreigner' or 'foreign.' Thais do not refer to non-Caucasian foreigners as 'farangs', though.
} 
itself would no longer work - hence the aptness of the odd-eyed cat metaphor. This may sound rather obscure, so let us scrutinize the many odd-eyed partnerships at CDW.

\section{Partnership of nationals}

A key policy at CDW is always, as Khun Karel put it, "local expertise with international standard.' In simple terms, 'local' meant Thai while 'international' meant Dutch/Western (as a touchstone for international standard). But what did the policy really mean in real terms?

The requisite need for local expertise was driven of course by the nature of the industry in which the firm operated. For one thing, in the construction and architectural industry (as in other professional fields such as law, medicine, or accounting), legal mandate always calls for professionalism that is locally certified. That was indeed the very first reason why Khun Geert needed the locally certified architect, Khun Chatoeran, to 'sign off' the initial project. However, legality was but the tip of the iceberg since local expertise also involved an on-the-ground familiarity with local practices, preferences, and quirks, some of which were too subtle for an outsider to recognize, let alone appreciate.

To give an obvious example, even though the Thai do not subscribe strictly to such belief as the Chinese 'feng shui' ${ }^{\mathbf{2 4}}$, they do observe a looser form of such practice. A building front gate needs to be positioned in such and such a way in order to induce stability and prosperity. Internal arrangement of different types of rooms and spaces should follow certain principles to ensure physical and spiritual harmony. One does not sleep with one's head towards the South or the West; else one may be subject to inferiority or misfortune (because another meaning of the Thai word 'South' is 'to be under' while that of 'West' is 'to fall'). Certain colours are not to be used for such and such spaces or purposes. And so on, so forth. Thus, it is not uncommon in Thailand to have a building torn down or renovated to 'correct' spiritual (not physical) mistakes. As a result, a religious-cum-spiritual ceremony performed on sites always marks the inauguration of a construction project, particularly a major one. This ritual requires an involvement of many experts in diverse specialized teachings, an occasion that would pose average foreigners quite a challenge to orchestrate ${ }^{25}$. No wonder CDW needed local expertise to help carry out its business. Ignorance or inobservance of such seemingly immaterial familiarity, or even unreasonable superstitious belief in some Westerners' eyes, could spell disaster. And this represented but one example of the many

\footnotetext{
${ }^{24}$ Feng shui is '(in Chinese thought), a system of laws considered to govern spatial arrangement and orientation in relation to the flow of energy ... and whose favourable or unfavourable effects are taken into account when sitting and designing buildings.' (Oxford University Press 1989, p. 674)

${ }^{25}$ As a reflexive note, during my childhood, I became involved in many such rituals as a helping hand to my mother's side business. In her spare time, she earned good fees for the arrangement of Brahmin rituals performed by my grandfather, who served his whole life as one of the Thai King's Brahmins. For this reason, I can claim first-hand 'understanding' of the significance ascribed to these rituals in the Thai culture.
} 
instances of local familiarity that played a subtle yet influential role in the daily operation of CDW.

On the other hand, the need for international calibre was partly a result of CDW's particular choice of its niche market. CDW's clients were major players in the global scene, the majority of whom are big name multinationals that needed to observe international standard in all its facets, particularly that of industrial, environmental, and quality control. The Dutch ownership of CDW in effect created a trademark of international standard for the firm. Indeed, as Khun Chaowalit put it:

When I deal with Western clients, I'm basically selling 'Westerness' if you know what I
mean. To be honest, I think some of the Thai engineers and architects may be better at their
functional tasks than their expatriate counterparts, well, at least better at those here in
Thailand for sure. But I can't sell Thainess to the 'farangs'. They wouldn't buy it. It's just a
fact of life and you've simply got to take it or leave it.

So, for many such projects, Khun Chaowalit needed to put upfront the appropriate expatriate professionals to secure the expatriate clients' trust. These expatriate professionals of course earned expatriate pay, which drove up project costs considerably. Still, the majority of CDW multinational clients were more than willing to pay higher prices for such a trademark. It did not really matter if in many cases the actual projects might make heavy use of the internal Thai expertise at CDW. First was to secure a project. And if it took the expatriate' stamp for that, so be it.

But international standard was in many senses more than just a trademark. As with local expertise, CDW's tie with Western know-how and practices represented also a unique quality of material value. In an industry where advance in technology is critical, Western experience and knowledge did give CDW a cutting edge. Khun Geert remembered how he needed to bring people in from the Netherlands to train the Thai professionals on CAD/CAM technology during the early years. Khun Chatoeran valued the Dutch-Thai partnership because he knew that it was the best way to tap on Western know-how to move Thailand forward. Khun Karel knew for certain that in some areas, such as quality control or the specialized field of water and environment, the Thai had quite a bit to learn from the Dutch. Khun Chaowalit also professed genuine respect for the Dutch expertise with waterworks:

You've got to admire the Dutch in this. They really know how to deal with dyke and dam and water. They're really the expert in things like irrigation, coastal protection, maritime, and so forth. And the Dutch experts in these area that come here really deserve our respect. They are 'the real thing'. And I'm certain that RHG Asia will win a lot of projects in the future because of the strong Dutch expertise.

And that was that. The Thai were good at some things and the Dutch were good at others. At CDW, they both needed each other's unique quality to create the unique odd-eyed identity. More, each needed to excel at their quality in order to reinforce the eventual unique identity, at least at the 'appearance' level, and more importantly at a more profound level. Still, the odd-eyed appearance could be a cause of unavoidable rift and tension. Khun Chaowalit and Khun Busara, for example, witnessed on a regular basis how such 'keeping up appearances' could cause resentment among the Thai staffs and create friction between the local and the expatriate. 
Looking from the Dutch expatriates' view, the higher prestige and pay were of course not much more than what they would earn in the Netherlands. It would make no 'Dutch' sense, at the very least, to expect them to be rewarded the same rate as the local Thai, unless of course you were one of the 'unDutched' who chose to adopt Thailand as your new home. At CDW, there was indeed one such local-hired Dutch whose position, rank and earning were the same as those of the local Thai personnel. But that was the 'price' he had to pay for wanting to be a naturalized Thai and stay in Thailand permanently with his Thai family. Most qualified Dutch professionals, though, would expect at least the same reward they would get in the Netherlands, if not more, for coming to work in a foreign country. With the cost of living in the Netherlands and in Thailand being starkly different, this situation would not change any time soon. And that was that.

Still, many fine Thai engineers and architects at CDW could not help feeling it was not quite fair for them to earn a lot less for the same type of work (that they were often better at) simply because they were Thai. Khun Busara sympathized very much with her Thai colleagues. They were quality people and their loyalty to their professions and the firm was unquestionable. Oftentimes, she understood their sentiment perfectly and it pained her to witness the undercurrent of this 'double standard'. She questioned whether the need to impress Western clients was justification enough for the double standard and even if it was, that still did not make the situation any less unfair. Khun Chaowalit couldn't agree more. He was a fine Thai engineer himself and he could hold his own with any Westerner in his functional capability. Yet, he could not have secured as many prestigious projects as he had done, if he had not had the 'Westerness' or 'farangness' as a trademark to market his ware. Trademark was nothing but appearance, of course. But it was paradoxically an appearance that was more than skin-deep.

It would have been fine, of course, if business would allow CDW to upgrade all Thai professionals to earn the same as their expatriate counterparts. Yet, that would surely render CDW's service ridiculously expensive and utterly uncompetitive in the Thai and Asian market. After all in any business, it was the bottom line that counted and Khun Chaowalit's job hinged on the kind of bottom line that necessitated the double standard. So, double standard it must be.

Odd as it is, the odd-eyed quality that CDW boasted was unavoidable as well as indispensable, even though it may be a cause for many concerns. As Khun Chaowalit put it, 'it's just a fact of life and you've simply got to take it or leave it!'

\section{Partnership of professionals}

Unfortunately, the inevitable friction between the Thai employees and Dutch expatriates was not the only tension Khun Chaowalit had to deal with. As a project manager, he also had to orchestrate the many departments within the group so that they worked in harmony and brought each and every project to a successful completion. He would not say it was a simple task. For one thing, he was constantly spinning at the 
centre of all functional divisions: conception \& design, budgeting, procurement, construction, interior work, and quality control. In effect, as a project manager, Khun Chaowalit was at the hub of the many partnerships of professionals and functions at CDW.

Of course, each profession had its own professional culture that underpinned the beliefs, values and norms of the community and influenced acceptable attitudes and behaviours. Tension naturally emerged whenever two or more professions or 'communities of practices' needed to come together and cross each other's comfortable zones. Hence, it was yet another crossing of cultures that Khun Chaowalit needed to turn into a partnership rather than a combat. Just like the partnership of nationals, this partnership of professionals at CDW was founded on the principle that each profession must excel at its own expertise while contending with and exploiting the unique expertise of the other professional partner(s).

Let's take the case of procurement and quality control as a lucid example. Procurement people's main concern was to keep to the budget and schedule. It was not that they were eager to sacrifice quality but their foremost 'bottom line' items were first the timely and correct delivery of materials and, second and most importantly, the cut-and-dried figures in the final project account. It was not unthinkable that cutting corner might come into play and that was where quality control needed to step in and have their say. The interplay between budget, time and quality control could easily make or break a project. Khun Chaowalit, again as a project manager, must make sure that an optimal balance was struck, with the least discord possible. Far from a piece of cake.

Then, there was another partnership of the engineers, the architects, and the interior designers, three closely related yet distinct professions who took great pride in their expertise - pride that could often turns into prejudice against the other professions. At the same time, each party had to have enough respect and confidence in the other's expertise in order to carry out their mutual assignment - turning conceptual design into physical reality. Again, this dictated a good balance of healthy self-esteem v. courteous trust in the other parties for the partnership to work. For instance, if a design did not work out quite well in the construction phase, who was to blame - the designer or the builder? As far as Khun Chaowalit was concerned, he would prefer that the blaming game was played off as seldom as possible. Yet, with such a threesome of esteemed and proud professions - again not a piece of cake.

These were but a few examples of the myriad partnerships of professions constantly going on at CDW. More, these myriad professional partnerships often crossed one another and complicated the situation even further. Given the countless professions that made up the CDW symphony orchestra - architects, designers, draftsman, engineers, foremen, mechanics, technicians, attorneys, accountants, quality controllers, and other specialists, Khun Chaowalit and his fellow project managers indeed needed to constantly exercise their conductor-like skill to create a harmonic project 
execution. Even for a maestro like Khun Chaowalit, this was still not a piece of cake, although he apparently must somehow make it his cup of tea.

\section{Partnership of organizations}

Finally, Khun Chaowalit stood yet at another crucial junction in CDW's operation, one between CDW and three indispensible external parties: the clients, the contractors/sub-contractors, and the authority. With all these three external parties, he had to orchestrate still another special partnership where all factions must uphold their functions, while contending with the views of the other partner(s).

Take CDW's relationship with the clients, for example. Convincing the clients to take up the best decisions and actions possible constitutes a hallmark of excellent consultancy. Coming up with proficient advices was critical but usually straightforward, because it was after all what CDW professionals were trained and qualified to deliver. The architects, designers, engineers, mechanics, and other specialists generally had no real difficulty delivering expert advices vis-à-vis their professions. Yet, necessary as smart advices were, they in themselves were certainly not sufficient. The clients had to see and agree that these were indeed 'smart' - a matter not as straightforward. For one thing, what about the old adage that 'the customer is always right'? Now, that is not always the case with consultancy. In fact, in the consultancy business, customers cannot always be right, else why would they seek the consultants' expert advice in the first place.

Nevertheless, a client was a client was a client, and consultants must always walk the fine line between upholding their expertise without offending the clients. They must confront and challenge the clients when the clients were not quite right, eventually convincing the clients to take up their advices. The ability to handle this oxymoronic condition was the key to consultancy business. It required a good interplay of confidence, trust, respect and assertiveness. Interestingly, Khun Karel, as CDW President, observed that the Dutch appeared to be better at this little game, particularly when it concerned Dutch or Western clients who were used to separating emotion from subject matter - yet another reason for the need to have expatriate involvement. Khun Chaowalit, on the other hand, also witnessed how in some circumstances involving Thai clients, a touch of 'Thainess' was often called for to establish good rapport. Still, for some other instances, a dose of opposite cultural tendency turned out to be more useful, such as when a stubborn Thai client would listen with less feeling of losing face to a blunt expert foreigner. Take the historic case mentioned earlier when Khun Chatoeran turned to Khun Geert to deal with a Thai client, for example. Khun Chatoeran suspected that as a Thai, the 'kreng jai' notion could make it awkward, if not impossible, for him to confront the Thai client with a dose of strong medicine. Khun Geert, in his Dutch cloak, could deliver the difficult message more easily.

All things considered, in most cases, a combined Dutch-Thai team proved to be the most effective for CDW. Whatever the case maybe, though, partnership with the clients demanded that CDW professionals must balance well the respect for their own 
professions, for their clients, and for the end results. These three distinct types of respect were not automatically in harmony and the Project Manager like Khun Chaowalit stood always at the centre of the balancing act.

The same applied to the partnerships with outside contractors and official authorities, two external parties CDW needed to constantly concur with who did not necessarily see eye to eye with the firm's agenda. Contractors, such as construction companies or other specialists, naturally had their own business agendas to tend to, agendas that might or might not be in line with those of CDW's. Yet CDW must work with them to ensure successful completion of projects, much in the same way as with the clients, only maybe in a reverse fashion. Finally, CDW must also deal with governmental agencies on a regular basis. The need to obtain a variety of permits alone meant that CDW must concur with many public offices, a transaction where local practice and international standard must form a harmonious agreement. Therefore, partnership with local authority became another balancing act of diverse organizational cultures.

Khun Chaowalit and other project managers functioned at the hub of all these partnerships: of national, professional, and organizational cultures. Their main instrument to orchestrate these partnerships was to convince all parties that they were all in it together - preferably for better rather than for worse, for richer rather than poorer.

\section{Partnership of ranks}

While Khun Chaowalit's job was at the hub of functional activities, Khun Busara's role at CDW put her at the centre of administrative and social acts. As a long-timer with the firm and the gatekeeper for the top Dutch bosses, Khun Busara had evolved into a key figure in the partnership of ranks at CDW. As executive secretary to the top bosses, she was often taken to be a de-facto spokesperson for the higher rank. Yet, Khun Busara also identified with the lower rank, because her position was more similar to theirs. In effect, Khun Busara was constantly the go-between, serving as microphone as well as speaker, and in a full duplex fashion so to speak, since she channelled messages in both directions simultaneously. Little wonder that Khun Busara found this strenuous role to be thanklessly rewarding - a rather curious affair that reflected yet another odd-eyed partnership at CDW. Again, a kind of orchestration must be in place to sustain this partnership of ranks.

Most strikingly, there seemed to be a strong correlation between ranks and other cultural groups, particularly national and professional cultures. Khun Busara noticed that at CDW, the Dutch, or the Westerner, were often in the higher ranks and the Thai were mostly in the lower ranks. Then, there were the subtle differences in status even among similar professional groups. Engineers and architects were regarded more highly than foremen or draftsmen, for example. There were exceptions of course, but the correlation between ranks and other distinct cultural groups was rather dominant at CDW. This complicated the issue further, because people had the tendency to muddle up the various identities of these diverse cultural groups. 
Khun Busara witnessed, for instance, incidents in which opinion of a Thai in a very high rank was dismissed by the top bosses a lot more easily than it would have been coming from a Dutch of the same rank. Was it because his national identity completely overshadowed his rank in the eyes of the top-ranked Dutch bosses? Then, there were incidents where she felt that her close connection with the top Dutch bosses caused some of her Thai colleagues to assume that she would always be 'on the Dutch side' because they were her bosses. What happened to her Thai identity then in this case? On the other hand, whenever she succeeded in persuading her Dutch bosses to agree with what the lower rank Thai employees wished, she would then be regarded as 'one of' the Thai rank once again. Still, that often came at the expense of her own bosses feeling slightly hurt because, in her words, they may feel that 'I was more passionate about my fellow Thai colleagues than about my own bosses.' It was all quite confusing, to say the least, when the partnership of ranks seemed to be tangled up with the parameters of all other cultural partnerships that were going on at CDW.

That was especially so when cross-interpretation came into play. Khun Busara observed such tendency in all ranks and other cultural groups. For instance, the Dutch could appear rather arrogant simply because they were Dutch. Would being Dutch automatically guarantee superiority the same way higher rank rightly do? Were they really superior because most these Dutch happened to be higher in rank, or were they simply being too proud of being Dutch? On the other hand, some Thais could be just as arrogant simply by virtue of being the host of the playing field. Since this was their country, they assumed they must have the right-of-way, even though they might be of lower rank. But then, take the case of the sole non-expatriate Dutch, who was regarded as 'the poor guy' by many Thai personnel simply because he was a Dutch who must suffer a Thai salary and rank, thereby standing out as an odd mismatch of rank and national identities. Why should he be pitied because he was in the same status as most Thais? Did that not imply that he deserved more simply for being Dutch? Now, whatever happened in this instance to the concept of rank? Was rank now redefined by national cultures rather than by its own merit? Yet, at the same time, Khun Busara found most Dutch bosses to be less authoritative than their Thai counterparts. 'The Thai bosses always think they are 'bosses' - that is, they are simply above you. But the Dutch bosses look at you more like their assistants, their helpers or colleagues.' When did rank really matter, in and of itself, and in what manner; or was there any formula to relate ranks to other cultural identities? - Khun Busara often wondered.

The partnership of ranks, even in the eyes of only one person at the hub of it, was far from a straightforward matter. At the very least, partnership of ranks certainly did not operate in a vacuum, much like all other partnerships. Interestingly though, rank seemed to be heavily correlated, rightly or wrongly, with many other cultural identities, making it a tough puzzle to sort out for Khun Busara and many others for sure. 


\section{De wereld draait door...}

Be that as it may, life went on at CDW, in spite of and because of the frictions and puzzles created by the odd-eyed identity of the firm. It was as it was and most at CDW had learned to live with or even thrive in it. Here and there, one may hear some jarring notes or see some out-of-step moves. But, after more than three decades of successful operations, CDW was certainly not out of tune or step in any major fashion.

De wereld draait door. ${ }^{26}$ And as the world turned, life went on. All things considered, it seemed to be a rather intriguing world and thriving life at the odd-eyed CDW the fact that the joint-venture ranked among the top in their industry in both the Thai and regional markets served as a testimonial to this assertion.

\subsection{Cultural crossing at CDW}

The story of CDW illustrates how the many levels of culture and the forces of numerous cultural groups or identities - national, professional, organizational and 'rank' cultures - could all interlace in a real-world setting and serve as an instrumental force of success, or failure, amid tension and confusion in one particular cross-border strategic alliance.

CDW thus represented a Dutch-Thai cross-cultural platform that thrived by weaving together the many intricate cultural webs to achieve a unique pattern of partnership which, metaphorically speaking, became its indispensible trademark.

\section{Multi-level cultural partnership as strategic trademark of CDW}

The most striking imprint of the origin and evolution of CDW was how cultures, in various shapes and forms, and their particular mode of crossing, served as a virtual trademark of competence for CDW. As a company that operated in an industry dependent upon perception and promise of competence, CDW needed to carry a prominent mark of distinction, something that conveyed past success as well as warranted future fulfilment. Success and fulfilment were of course in the eye of the beholder and since CDW had many groups of beholders, it needed to carry a trademark of competence that had many facets to attract the various beholders.

Hence, multi-faceted national culture was the first distinctive trademark of competence for CDW. For Western or Dutch clients, Dutch ownership served as a trademark of international competence; for Thai clients, Thai involvement that of local fluency. For all clients, its Dutch-Thai alliance afforded a trademark of desirable combined expertise. In all these instances, a particular national culture, or a unique aggregate thereof, served as a trademark of an exclusive type of proficiency, the reason Khun

\footnotetext{
${ }^{26}$ Literally, this translates into 'The world continues to turn'. Figuratively, it has the same connotation as the English expression: 'Life goes on'. The dramatic use of this expression here was definitely inspired by the daily Dutch television talk/variety show De Wereld Draait Door which was running successfully at the time of this writing.
} 
Karel, the President, emphasized the value of a 'combined' team, which epitomized the unique trademark of the total sum of CDW's competence.

Professional culture was of course another distinct trademark of CDW's competence. Granted, most, if not all, professionals at CDW were legally certified; yet legal certificate was but one stamp of qualification. Generally, each profession had its own culture, which bonded the members of each profession to one another and guarded them against those outside of their circle. The 'guarding' function of professional culture trademark had a decisive role at CDW because the firm operated in a consultancy business where professional advices must carry dominant weight when set against clients' stance. It was not only the material capability of the professionals, but their authoritative aura and clout that made them an equal partner, not a mere servant, to the clients. Hence, CDW had to retain its 'professional' eye colour on par with that of its client - another odd-eyed instance.

CDW also needed to blend many organizational cultures in its simultaneous dealing with clients, suppliers, contractors and governmental agencies. Each of these organizations had its organizational culture that was a particular blend of internal and sectoral cultures, at the very least. The competence of CDW as a consultancy must be constantly upheld by successful management of the partnership of its own organizational culture with those of these other establishments. As mentioned earlier, as a consultancy, CDW had to maintain its own standards without denting the prestige of these other business partners and clients - once more, an odd-eyed sort of interplay.

However, the odd-eyed trademark also carried with it certain complications, partly because the odd-eyed personality of CDW was a distinct case of cultural crossing. The uniqueness of the odd-eyed identity of CDW stemmed from the contending yet uniting condition of various competing cultures. CDW needed this particular instance of crosscultural identity but at the same time it created constant tension and puzzling interpretation, illustrated by the many 'unanswered' and some 'unanswerable' questions in the mind of Khun Busara regarding the entanglement of the many cultural groups and their simultaneous and conflicting forces within CDW.

The questions are thus - how did CDW maintain its odd-eyed personality and what made it work despite the tension and confusion that came with it? The simple answers would be: CDW maintained its odd-eyed trademark because it needed to, and this puzzling identity worked simply because it must. Since CDW needed the odd-eyed personality, with all the tension and the contention that come with it, everybody involved needed to make it work, for survival's sake at the very least, and for prosperity's sake at the very best.

These two misleadingly simple, or some may even say simplistic, answers are in fact a kind of epiphany, as they reveal insights that are deceptively obvious, yet simplistically powerful. They underpin two fundamental properties of effective crosscultural interface: context and instrument. 


\section{The significance of context}

Because the odd-eyed identity was the cultural trademark of competence that CDW needed for its chosen business, the firm's context in effect defined its operative identity and qualified its eventual livelihood.

Consequently, the story of CDW illustrated vividly how significant context is in any cross-cultural encounter. In the final analysis, what matters is the total sum of a particular context. To wit, there are no two CDWs, so to speak, and if we want to understand what is going on at a particular instance of cross-cultural interaction, we need to take its general and specific context into account, simultaneously. Although all cases of a cross-cultural platform share the same general properties, the eventual identity of each setting is unique unto itself. This makes an analysis of context indispensible if we were to truly understand a particular case of cultural crossing.

In the case of CDW, the context defined the definite type of partnership and the exact mode and nature of cross-cultural condition for the alliance to function well. First and foremost, the combined context of its industry and its niche business made the cultural odd-eyed quality a necessary trademark of its competence. Additionally, other conditions and entities that made up the joint-venture all contributed to how CDW was what it was. Finally and just as importantly, the context also defined the eventual effectiveness of the cross-cultural interaction itself. In brief, if the eventual condition of a certain cultural crossing contributes to the prime objective of its existence, then it is effective, despite and because of all the oddities and difficulties it creates.

As such, the many types of partnership that co-existed at CDW exemplify the very property of intricate cultural webs. Here again at CDW, we see a cross-cultural platform that was made up of simultaneous 'crossings' of different shapes and forms of complex cultural units - individuals as well as groups. The complexity of this labyrinthlike characteristic is inherent in any cultural entity or condition that is made up of more than one culture, in CDW cases at least four possible major cultural groups (national, professional, organizational and rank). This characteristic is inevitable and defies any definitive characterization further than that it is complex and infinite. Yet, the many actors at CDW appeared to be able to handle this complexity with relative success. What was the 'secret' that help them manoeuvre their way in this labyrinth?

A possible clue may lie in how the successful actors related their experiences. Particularly striking was Khun Chaowalit's insistence on the 'take it or leave it' mentality. It seemed that those who had chosen not to leave it eventually learned to take it, one way or another. If so, what was it that they needed to 'take'?

In CDW case, once the particular form of partnership was demanded and defined by the context, it was a simple matter of survival and prosperity for the firm to make it work. Partnership, in essence, works on the basis of the saying: 'It takes two to tango'. On the surface, this may sound rather cynical; at a deeper level, it is simply pragmatic. There are two sides to the expression. On the one hand, it carries the usual critical connotation that in any detrimental dispute, both parties are equally responsible for the negativity. On the other hand, it signifies a realistic recognition that in a successful 
joint endeavour, both parties need each other to complete the mutual undertaking, because each lacks what the other has. At CDW, both meanings were at work and both were what all actors had to 'take' as simply a fact of life.

This 'fact of life' was accepted to various degrees by the many actors at CDW. Most impressive, though, were those who not only accepted the necessary evil of partnership, but also learned how to wield its double-edge sword just so the possible damages were minimized and the potential benefits maximized. And the key to their strategy resided in the exercise of appropriate cross-cultural instruments.

\section{The value of cross-cultural instrument}

The tension detected at CDW could only take on consequential effect if both parties allowed it. As we see, although the tension was certainly there, a mechanism must have been in place that prevented it from degenerating into total negativity. In the case of CDW, one very clear such mechanism was in the form of what is theorized in the proposed grounded theory as a 'mediator'. The precise property of a mediator, and its relation to the other three forms of cross-cultural instruments is elaborated in the following chapter where the theory is explicated. A preliminary observation of the various mediators active at CDW serves as a prelude to the said explication.

A mediator refers to any instrument that acts as a 'go between' for difference parties in order to render a mediate effect (Ulijn and St Amant 2000). The need for a 'go between' arises from some fundamental differences that call for a certain level of synchronization before the parties can interact with a tolerable level of friction, incompatibility or detrimental side effect. A mediator comes in a variety of shapes and forms, each performing synchronization in different manners. Various mediators were active at CDW. First were the human mediators in the figures such as Khun Busara and Khun Chaowalit. Both actors operated at the centre of critical activities in CDW and both acted as mediators for the many forms of partnerships at CDW as illustrated in the case. In the case of the co-founders - Khun Geert and Khun Chuchawal - however, the mediator took the form a 'common denominator' that was the mutual need and appreciation in each other's contributions to the partnership.

The importance of mediator exemplified in the story of CDW provides a strong hint at the significance of key instruments in cross-cultural interface. As will be expounded in the following chapter, three other types of cross-cultural instruments are theorized, making up the four types of key instruments - controller, hydridizer, and adaptor each serving different purposes and suitable for different cross-cultural conditions. The case of CDW illustrates the critical role that the right instrument can play in a crosscultural interaction. 


\subsection{Final words}

\section{Khun Geert}

I should emphasize one more thing. If you're working in our type of business - services - you have to find a good local partner. We have competitors in our field of business who failed because they didn't have the right local partner. I said before, in the beginning, we were lucky to have a very good local partner in Khun Chatoeran. Especially in our type of business ... when you provide service, there's an element of trust and knowledge of local partner that you need.

\section{Khun Karel}

Mutual respect is always what I try to maintain. I always convey to both the Dutch and the Thai that we cannot do without the other.

\section{Khun Busara}

I myself like to have a variety of styles. I mean, sometimes I'd like the bosses to tell me strongly when I'm wrong too. With the Thai style, it's good for the purpose of 'Pok Krong', that is to set a proper environment for authority and control, which you need sometimes. It makes you feel alert. But I think the Dutch more respectful style is also good. So, I like it both. It really depends.

\section{Khun Chaowalit}

For the Dutch, what they're interested in is not to be the best but to make the best of what they have. They don't focus on being top leaders like having top automobile brand such as Porsche or building top aircraft like the Concorde. What matters for them is the real bottom line. As long as it's a win-win situation, the Dutch are happy.

T HUS, THE CASE OF CDW EXEMPLIFIES FURTHER SEVERAL KEY CONCEPTS IN THE GROUNDED THEORY IN this study, not only by the few answers it suggested but also by the many questions it raised. These questions became part of the foundation for the key outcome of this study - a grounded theory of cross-cultural intelligence, the very subject of the next chapter. 



\section{Chapter 6}

\section{XCQ AMID INTRICATE CULTURAL WEBS}

\section{A grounded theory}

Something old, something new

Something borrowed, something blue

And a silver sixpence in her shoe.

Anonymous

old English rhyme

circa England's Victorian Era

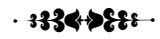

It was a wonderful sensation, this. He felt like a king when he was able to harness the power of wind and waves and set them to do his bidding. Not that it was quite so entirely in his command. In truth, he knew that Hamadus had been right many years before, when he had told Isok that the real skill of a mariner lay not in trying to force the vessel or the seas to do his bidding, but in seeing how the sea and his ship wanted to behave, and persuading each to permit him to go as he wished.

Michael Jecks as part of a monologue in his fiction - The Outlaws of Ennor

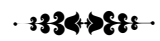

How many units of human compassion And how many grapes went into the wine How many illusions were sacrificed wantonly How many people have looked for a sign?

$$
\text { [...] }
$$

How many truths have run through our fingers And how many credos ... . Believing in mine How many have led into unthought of dangers And how many grapes went into the wine? 
Reflection 16: 'And how many grapes went into the wine?'

DURING the course of this study, I often turned to my Dutch partner for help with clarification of some Dutch words, phrases, idioms, customs and habits. This gave me assurance that I would be less prone to interpret wrongly the language and custom that are not my native.

One time, I couldn't figure out from the audio record precisely a Dutch word that Khun Kees used as a nickname for Khun Maleewan. I asked my Dutch partner what a Dutch term would be that described somebody who always smiled or laughed. We finally figured out it was the word 'lachebekje' that Khun Kees used. My partner then asked me what it was all about. I said it's a word an informant used to describe a Thai employee. My partner then remarked, 'But all Thais are lachebekjes!' And, how did I respond to that? I laughed, of course, like most Thais would do in such a situation.

But then, my Dutch partner's response surprised me. He started to laugh with me and then his laugh softened into one of those 1001 Thai smiles, a smile not at anything funny, but at something amiably annoying. After years of our constant bickering over the stupidity of Thai smiles, we ended up smiling at the absurdity of such squable, together.

I didn't quite realize the meaning of this experience until I was well into my theorizing. And the realization came at the most unusual moment when I was reading a passage in a mystery novel - a passage that has nothing to do with cross-cultural intelligence but another type of intelligence altogether. It was a passage when a character reflected on his sailing skill. Yet, the description that 'the real skill of a mariner lay not in trying to force the vessel or the seas to do his bidding, but in seeing how the sea and his ship wanted to behave, and persuading each to permit him to go as he wished'seemed to describe exactly what I saw in the actors of the cases. Those who professed comfort with cross-cultural interfaces were ones who had a power of observation, understanding and persuasion, to assess the context, appreciate the meanings, and persuade all involved, including themselves, to permit them all to do what they mutually wished, against all odds.

I realized then that the core category of my little theory was neither 'knowledge' nor 'knowing' about culture and cross-cultural interaction, but a little bit of both knowledge and knowing, plus a huge dose of understanding - a kind of intelligence. The power of metaphor and analogy helped me to piece together concepts, ideas, insights, intuitions and epiphanies that seemed to come from all over the place, even the most unlikely nook and cranny.

In this manner, I could say that the theory and my theorizing were woven from 'something old, something new, something borrowed, something blue' and even 'a silver sixpence' in my shoe. Or, borrowing Beer's analogy, there were many, many grapes of grounded evidence from all sorts of informants - formal, informal, scholarly, literary, and accidental - that went into this wine of grounded theory I now proceed to present.

I humbly hope the wine tastes good to my readers and does not intoxicate them all too much. 


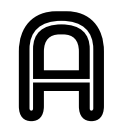

CCORDING to Weick (1995), 'What TheORY IS NOt, THEORIZING IS' - THIS CHAPTER REports a theorization of a grounded theory of cross-cultural intelligence, thereby fusing the product with the process - theory with theorizing, simply because as prominent scholars have keenly observed, it is rather impossible to distinguish the two (Dubin 1976, Langley 1999, Weick 1989, Weick 1995). This qualification, for one thing, reflects the humility of theoretical claim made here. Both the theory and theorizing I present in this manuscript are work-in-progress at best, elementary at worst - when put against the larger effort of theorizing on CQ. Although what is strived for is the best, the worst may not be too disheartening, given the complexity of the subject at hand.

The theorizing process that went on in this study solidified when the second core category emerged, a process which started with an attempt to find a good 'link' between the two elementary themes of culture and learning. That query slowly evolved into the concept of 'cross-cultural intelligence', whose moment of 'epiphany', if such a hyperbolic expression may be indulged, came with a reading of Clifford Geertz' assertion that, 'it is not necessary to know everything in order to understand something' (Geertz 1993, p. 20, emphases added).

That is what distinguishes knowledge from intelligence. The essence of intelligence is 'understanding' and it was this appreciation that solidify the two core categories in the theory expounded here. Knowledge, even a thorough one, of a something does not automatically translate into the ability to relate to that something in an appreciatively effective manner. It takes true understanding of something for one to be able to relate to it in a manner that is in any way rewarding. Intelligence thus has more to do with genuine understanding than sheer knowledge. As such, cross-cultural intelligence fuses together the two concepts of culture and learning - it epitomizes an understanding of what culture is and exploiting that understanding to operate well under its intricate force.

This chapter elucidates a theory of cross-cultural intelligence that is the key finding of this study. I name this theory 'cross-cultural intelligence (XCQ) amid intricate cultural webs' to succinctly denote the two core categories of the theory - cross cultural intelligence (or XCQ) and intricate cultural webs, as well as their relationship - amid.

\subsection{Cultural crossing}

As introduced in Chapter 1, the term 'cross' has definitive significance because it accentuates the very focus of the core theory emerged from this study. XCQ is a capability and strategy that is needed for a cultural unit to handle the complex intricate cultural webs that characterize its cultural identity while that cultural unit operates yet in the intricate cultural webs that also define the condition of any cross-cultural interface. The emphasis here is on the significance of 'cross' - hence cross-cultural intelligence, rather than simply cultural intelligence. The choice of the letter $X$ in the abbreviation exemplifies this emphasis, which marks the first subtle distinction be- 
tween the concept of XCQ and that of cultural intelligence or $C Q$, an issue to be duly expounded.

The locus of XCQ is thus not in the nature of its focussed entity - namely, intelligence about culture, but in the nature of its interactive feature - that is intelligence when dealing with cross-cultural situation. Cultural intelligence, as defined by prominent theorists on the subject, is fundamentally about the ability to react effectively in an unfamiliar cultural context (Earley and Ang 2003, p. 59, Peterson 2004, p. 89, Thomas and Inkson 2003, p. 62). By comparison, cross-cultural intelligence is the ability to operate successfully in a context where the crossing of more than one culture is the salient condition. Unlike cultural intelligence, cross-cultural intelligence puts emphasis on the implications of the interaction, namely crossing, of cultural forces, rather than on the necessity to accommodate or adapt to one particular cultural influence. Granted, this is fundamentally a difference in focus and not in substance. The two concepts of $C Q$ and XCQ are very much related and lead to complementary implications. In this respect, it is fair to characterize cross-cultural intelligence as a sub-set of cultural intelligence, since XCQ is certainly part of the ability of a cultural unit to react effectively in an unfamiliar cultural context - one when various cultures come in contention. Yet, the emphasis on crossing bears distinct connotation.

\section{Modes of crossing in cross-cultural condition}

What then is the importance of the crossing factor in the proposed theory? First, simple dictionary definitions of 'cross' actually denote several subtle modes of interaction of which clashing is only one. When applied to crossing of cultures, in reality many possible conditions can result. This study evidence suggests four distinct modes of crossing when cultures cross or interact: clashing, reciprocal, unification, and variation. These modes are summarized in Diagram 6.1 which serves as a framework for subsequent discussion.

The first property when cultures cross relates to its 'dominant catalyst', which refers to the primary force in the interaction. This signifies what the actors identify as the salient ingredient of a particular cross-cultural condition - the initial or primary engine that motivates their interpretation of the thoughts, feelings, and acts of themselves and others within that cross-cultural context. Next, the property 'predominant interaction' refers to the most prevailing activity in an interface, which correlates strongly with the dominant catalyst. Third, the property 'key instrument' relates the primary mechanism used in order to carry out the predominant interaction and bring about the possible steady state resolution. Fourth, the property 'steady state resolution' signifies a state of a cross-cultural condition that is sustained over a certain period. Steady state denotes a sustained but not static condition because a certain cross-cultural phe- 
nomenon may start in one mode but later develop into another mode altogether. ${ }^{27}$ Finally, a mathematical analogy for each of the three modes serves a simple, intuitive inferential device.

Diagram 6.1: Taxonomy of cross-cultural condition

\begin{tabular}{|l|c|c|c|l|}
\hline \multirow{2}{*}{ Key properties } & \multicolumn{4}{c|}{ Modes of cross-cultural condition } \\
\cline { 2 - 5 } & clash & reciprocal & unification & variation \\
\hline dominant catalyst & difference & mutuality & affinity & empathy \\
\hline predominant interaction & conflict & exchange & hybridization & $\begin{array}{c}\text { adaptive } \\
\text { response }\end{array}$ \\
\hline key instrument & controller & mediator & hybridizer & adaptor \\
\hline steady state resolution & $\begin{array}{c}\text { separation, } \\
\text { division } \underline{\text { or }} \\
\text { assimilation }\end{array}$ & aggregate & hybrid & chameleon \\
\hline mathematical analogy & $\begin{array}{c}1+1=1,1 / 2 \underline{\text { or } 0} \\
\text { nyyyyy}\end{array}$ & $1+1=2$ & $1+1>2$ & $1+1=\infty$ \\
\hline
\end{tabular}

Note: Author's conceptualization based on study evidence.

Four important points should be made regarding the proposed taxonomy. First, the theorizing here involves no underlying judgement about the modes of cross-cultural condition: the taxonomy simply reports the range of options and possibilities indicated by evidence. Thus, any assessment of one mode's superiority to others is relative to the situation. What is informative and valuable is an understanding of what contributes to a particular situation's engendering of one mode rather than another and the consequences of such a development. Second, rather than deriving an exhaustive list of all possibilities, which would render the taxonomy redundant and cumbersome, the choice of the four modes indicates the range of possibilities that may characterize a cross-cultural condition. Third, these four 'representative' modes are collectively exhaustive, yet not mutually exclusive in a single cross-cultural situation (denoted by the broken lines in the diagram): that is, at least one mode must exist at one point while more than one mode may (and usually do) co-exist in a cross-cultural context or within any type of intricate cultural webs. The key is to discern what mode is the dominant

\footnotetext{
27 The terminology 'steady state' follows its use in the discipline of computer simulation in general and discrete event simulation in particular. Because of its relative neutrality, I prefer this term instead of its alternative that is favoured in economics - 'equilibrium'. However, I use the term steady state in a very broad sense and it thus does not embrace all the fine properties and implications of the terminology as used in the above two disciplines. As a reflexive note, the choice of 'steady state' was likely influenced by my former training in the discipline of discrete even simulation.
} 
one for a particular phenomenon under analysis and what that entails. Last, crosscultural modes are not static; rather, they are forever proceeding, or metaphorically being woven, although not necessarily as a linear progress and more likely in a weblike fashion. These four notes on the taxonomy of cross-cultural condition, particularly, the two last points, are revisited in detail in Chapter 7 where the case of ZIA serves to illustrate the above characterization of the variant modes of cross-cultural conditions.

\section{Clashing cross-cultural condition}

The clashing mode is one in which difference denotes the dominant catalyst and conflict often emerges as the crux of the interface. This is the most recognized and recognizable of all the modes because of its vivid nature and persistent prevalence. Indeed, this is the mode identified as the most significant by many prominent cultural theorists such as Hofstede and Huntington (Hofstede 1980, Hofstede 2001, Huntington 1993, Huntington 1996a, Huntington 1996b). In this mode, difference between cultures significantly, if not solely, influences how actors interpret and act. By 'difference', under an interpretive analytical lens, is the difference in meanings given to concepts, entities or acts.

The fixation on difference often, though not necessarily always, leads actors to contemplate conflict, because one meaning will want to exert its force over that of its counterpart. When this happens, the key instrument is generally a sort of controllersomething that renders one party the ability to govern the situation in the direction of the meaning it subscribes to. A controller can be in any form that ranges from formal authoritative power to subtle manipulative tactic. Whatever form a controller takes, its main effect is to establish the meaning in the one culture that the party wielding the controlling instrument identifies with as the governing meaning of the cross-cultural situation. In the clashing mode, there are three possible steady-state resolutions: separation, division, or assimilation. Separation transpires when actors from all involving cultures hold on to their subscribed meanings and go their separate ways, terminating the cross-cultural condition altogether. Division results when the differing actors from different cultures maintain their own meanings but still operate in the cross-cultural context in which conflict persists. Assimilation occurs when one cultural meaning totally subsumes the other(s), thereby effectively eradicating the forces of all other competing meanings for all actors involved. As far as mathematical analogy goes, the clashing mode is analogous to when one plus one could eventually yields one, onehalf, or zero.

There are numerous everyday examples of the clashing cross-cultural mode. The one identified by Huntington, for example, is believed by many to characterize today's world affairs, following Huntington's thesis (Huntington 1993, Huntington 1996a, Huntington 1996b). The clash of civilizations theory, in brief, predicts international relations to operate with cultural difference as the key catalyst and conflict persists and characterizes the relationship among major cultures or civilizations of the world. Hofstede's work provided countless examples illuminating how difference among cul- 
tures in organizations and societies could result in vivid tension that leads to either one of the three steady-state resolutions of assimilation, division, or separation (Hofstede 1980, Hofstede 2001, Hofstede and Hofstede 2005).

To delve into the three clashing resolution states as revealed in this study's cases, I will start with the easiest mode to detect, that of separation steady state. A small incident when ADT decided to terminate the Taiwanese contractors at the beginning of ADT entry in Thailand is a typical example of a clashing mode that ended in separation. The irreconcilable difference in this situation lied in the opposite meaning of 'professionalism' between the new Dutch owners and the inherited Taiwanese personnel.

The story of TMF, portrayed as a vignette in the last chapter, provides an example of a clashing mode of cross-cultural condition that prolonged in a steady state of division. Although no dramatic conflict was obvious in this vignette, the underlying condition of the Thai office of TMF was still basically of two dominant national cultures, Thai and Dutch, existing together side by side in a divisional manner. Khun Herman, the Dutch MD, looked at issues and exercised his functions based on a Dutch view of organization and management. He concentrated mainly on putting a Dutch management imprint on the company's Thai division, primarily through his 'prized' employee, the Thai Export manager. Yet, when looked closely, the rest of the company in Thailand operated more or less according to a Thai's view of organization and business. The two local sale executives ran their sale activities not differently from their counterparts in other Thai companies. At the same time, Khun Herman and his prodigy the Export Manager, managed the regional side of sales and marketing according to how Khun Herman and his Dutch colleagues would. Thus, TMF as narrated in the vignette was operating under a divisional clashing mode, because all parties involved appeared to be quite steadfast at how they wanted to function in the manner familiar to them and at the same time revealed underlying frustration or tension at how other actors chose to act in 'different' manners. Yet, this underlying tension had not moved the opposing actors to openly superimpose their preferences on the other parties. They simply kept on functioning in their own chosen manners in a 'together apart' fashion - each serving the same company but in his or her own way.

While TMF was in a divisional steady state clashing mode, ADT offered yet another clashing example, that of assimilation. The crisis episodes of repeated 'mistakes' in handling customer complaints resulted in an imposition of the company's sacred policy regarding communication with customers. It seemed most likely that if and when the same 'incident' were to happen one more time after two repeated warnings, ADT's top executives would certainly exercise the 'three strike and you're out' policy, implying a sort of assimilation into the Dutch's business philosophy. On the other hand, there were other elements in the case which suggested that this seemingly clashing mode could also inspire a development within ADT's cross-cultural environment that later altered the properties of the dynamics such that the cultural crossing condition might take on a different mode. Again, only a revisit of the company for further case research would reveal the actual development. As the case stood in the narrative, though, ADT 
presented a cross-cultural condition that was very much in the making, since other cross-cultural modes could also be identified in the case. As such ADT also serves as an illustration of how different cross-cultural modes could co-exist in one single situation.

\section{Reciprocal cross-cultural condition}

The reciprocal mode has mutuality as its main force. That is, in this mode, the focus of the actors involves some kind of mutual interest, desire, need, goal, and the like. Thus, mutuality refers to a common target that can only be achieved by having actors from the different cultures contributing inputs that are unique to their own cultures. Such a situation occurs when actors from each of the cultures have what the others do not have by virtue of their distinctive cultural heritage and identity. In this crossing mode, the focus of the interaction is mainly exchange, meaning straightforward give and take, where some sort of mediator is the key instrument. As the name implies, a mediator refers to any instrument that acts as a 'go between' for difference parties in order to render a mediate effect, analogous to a physical bridge, so to speak. In the reciprocal mode, actors from one culture give what those from other cultures need but lack, and vice versa, in order for all to achieve a mutual target - hence the label reciprocal. The steady state resolution of a reciprocal cross-cultural condition is a straightforward aggregate of cultural meanings that retains the essence of their original cultural sources, without critical change or alteration. Metaphorically speaking, this is an uncomplicated case of one plus one equal two, as in basic mathematics.

This second crossing mode is less easily recognizable but exists more often than most people realize. The case of CDW, the odd-eyed cat, is a lucid example of such a cross-cultural condition. CDW thrived on a reciprocal cross-cultural condition because its key strategy and trademark, local fluency with international expertise, was a mutual target of all concerned and can only be achieved by having each of its key cultural groups, particularly the national cultures Dutch and Thai, retaining or even brandishing its own cultural essence side by side - an issue already analyzed at length in Chapter 5.

\section{Unification cross-cultural condition}

In the unification mode, actors' affinity with the meanings in the other culture denotes the dominant catalyst. This mode is, in a simple sense, the reverse of the clashing mode and one step beyond the reciprocal mode. In this mode, actors in a culture discern that actors from the other culture have different meaning than that in their own, yet find it to be more admirable and desirable. Thus, in this mode, what is reciprocal is a fundamental affinity between actors from different cultures, each admiring, not merely in want or need of, elements or meanings in the other culture that do not exist in their own. In this mode, the predominant interaction is hybridization, because actors will attempt to assume the desirable meaning and make them an integrated part of their system of meanings. For this hybridization, a sort of hybridizer is naturally the needed instrument. A hybridizer signifies a mechanism that fuses various competing meanings into one new system of meanings. Such fusion evolves from genuine appre- 
ciation and aims at creating a new fused set of meanings hitherto not in existence. A hybrid entity - a novel entity entirely - then emerges as a result of this cultivation. Metaphorically speaking, this is a case when the new whole is greater than the sum of its parts, that is, one plus one is greater than two.

A cross-cultural unification and its hybrid creation may be more common in everyday life than most would imagine. An immigrant, who is well received by and has integrated well in a new country, is a good example. Such an individual has developed a new national cultural identity, at his own individual level, that is neither his birth culture nor the adopted one, but actually a fusion of both. His new cultural identity reflects some cultural traits from his own motherland, some from the adopted country, and some totally new but with influences from both. A successful mixed marriage is another everyday life example. In such a household, elements from all involved cultures blend together to form a new unique household, a hybrid cultural familial group.

From the cases in this study, several examples of a unification cross-cultural condition were present. As an individual cultural unit, many of the actors in the case have actually transformed their national cultural identity to be a hybrid one. Khun Pim of ADT and Khun Geert of CDW, for example, professed deep affinity with certain elements of the Thai culture that were starkly different from, or even opposing to, those in the Dutch culture. Khun Pim admired the lifestyle of the Thai culture and developed a preference to the Thai meaning of work and play that convinced him to decide to settle down and retire in Thailand instead of going back to his home country. Similar analysis can be applied to Khun Geert who was eventually as comfortable in Thailand as in the Netherlands. Khun Danny of the next case ZIA, on the other hand, was a Belgian whose affinity with the Dutch work ethic and business prowess had turned him into a unique unDutchable Belgian. Each of these actors in effect assumed a new cultural identity which was neither Dutch nor Thai, but a hybrid of both. At an organizational level, ZIA served as a case of cross-cultural condition that showed some elements of a unification mode, although more in a camouflaged manner - a key analysis of the narrative in Chapter 7.

\section{Variation cross-cultural condition}

The fourth and last of the cross-cultural modes is perhaps the most intriguing one. This cross-cultural mode emerged most subtly in the course of my theorization. In the variation mode, actors' empathy with the meanings in the other culture denotes the dominant catalyst. By empathy is meant a deep understanding or appreciation of the meanings an actor discovers in cultures unfamiliar to him or her. It must be emphasized that empathy does not necessarily imply or automatically equate to affinity. Empathy signifies understanding as if one were 'in the shoes' of those actors in cultures other than one's own. This understanding may or may not inspire affinity but it certainly leads to a particular pre-dominant interaction mode of adaptive response. Simply put, an adaptive response is an outward manifestation that is adapted to accommodate the meanings as defined by cultures other than one owns. An adaptive 
response serves diverse purposes and varies according to context, hence the name variation for this cross-cultural mode. An instrument that enables this adaptive response is an adaptor, which, analogous to the biological function of a literal chameleon, enables actors to create a kind of chameleon-like ability to adjust his or her response to suit a certain environment and reach a particular purpose. Hence, the steady state resolution of this cross-cultural mode is a chameleon state, which is virtually infinite in its manifestation - the case of the mathematically intriguing one plus one equals infinity. An elaboration of the key metaphor governing this mode of crosscultural condition should make the above statements more meaningful.

That metaphor is that of a 'chameleon'. This metaphor has been used in the literature with regards to the issue of social and cultural interaction and the concept of the variation mode here indeed echoes elements in this body of literature (Chartrand and Bargh 1999, Earley and Peterson 2004, McCaig 1996, Pollock and van Reken 2001, Thorne 2000). In addition, insights into the nature of the actual creatures, the chameleons, and their behaviour from biological field provide further theorizing acuity (Cooper 2002, John A 1981, Roach 2005, Starrett 1993, Stuart-Fox and Moussalli 2008). To begin with, chameleons are known to have a special ability that enables them to change their skin colours in response to the environment in which they find themselves. Biologists have explored both the mechanism and explanation behind this fascinating capability (Cooper 2002, John A 1981, Roach 2005, Starrett 1993). Three reasons are now established to explain the biological purpose of why chameleons would change their skin colours. The first is perhaps the most known and often misleadingly believed to be the only reason, that is the camouflage or mimicry response as a way to 'blend in' with the environment, either to hide from predators or deceive preys (Starrett 1993). However, recent studies have unveiled two other, even more insightful reasons for chameleons' colour changes. It appears that chameleons change their skin colours also to increase their comfort level within an environment, such as turning their skin darker to absorb more heat and light in cold temperature (Cooper 2002, Roach 2005). Lastly, it has also been established that chameleons use variation in their skin colours to communicate with other chameleons, for example, to signal submission, aggression or sexual desire (Stuart-Fox and Moussalli 2008).

Drawing from the above biological understanding of chameleons' behaviour, a 'cultural chameleon' in the proposed taxonomy is theorized to also exercise adaptive response in order to either blend in, induce comfort, or communicate. The most wellknown use of the term 'cultural chameleon' is associated with the study of a particular type of individuals - the 'third culture kids' or 'global nomads' - those who have grown up in a culture or cultures other than that of their parents' native land, such as a child of a career diplomat (McCaig 1996, Pollock and van Reken 2001). Pollock and van Reken, for example, explicated how third culture kids develop and use their cultural chameleon ability as a survival as well as comfort inducing tool (Pollock and van Reken 2001: 92-93). Chartrand and Bargh's study in social psychology investigated how subconscious social mimicry is exercised as "a kind of natural "social glue" that produces 
empathic understanding and even greater liking between people, without their having to intend or try to have this happen' (Chartrand and Bargh 1999: 897). More specifically to the theme of culture and learning in organizations, Thorne used the term 'cultural chameleons' to characterize how, in an organization, multiple professional cultures 'are described as coconstitutional: existing alongside each other in a relationship of asymmetric complementarity' (Thorne 2000: 326).

To date, the term 'cultural chameleon' remains comparatively new and its used relatively informal and mainly metaphorical. Evidence in this study, however, suggests that this particular steady state resolution of a cross-cultural condition carries a farreaching signification, particularly as distinct from a straight cultural aggregate or a cultural hybrid. In a cultural aggregate, such as the odd-eyed CDW, strong preservation and manifestation of original cultural identity is the key, whereas in a cultural hybrid, a whole new cultural identity is cultivated. By comparison, a cultural chameleon retains its original cultural identity, while displaying outward cultural indicators that accommodate variation in environment. Because the purpose of this adaptive response can be either to simply blend in artificially, to raise comfort level, to communicate, or indeed to achieve all of these purposes, a cultural chameleon could be a cross-cultural condition that is the most versatile, yet also the hardest to derive and sustain. The following narration by Khun Fons van Beek (of the next case, ZIA), paints a vivid example of such an adaptive response of this cultural chameleon ability:

\footnotetext{
I always have the honour to be the first one to sing [at the company's social Karaoke session], of course. I like it. At first I feel embarrassed, of course. But now it's OK. A lot of the Dutch they hate it in the beginning, but then ..., and this is something amazing about the Thai people I find. They like to do this kind of things in public. I think not many people in the Netherlands would like to go in public and sing or even say something in public, unless of course they are in a good position to do so like they are a performer or public speaker. But in Thailand, you can pick out anybody, put him on a stage, give him a microphone and without any shame, he'll be able to talk to the crowd and sing, without any shame, no problem at all. It's quite surprising about the Thai people.

First time it happened to me, I was shocked. I felt very, very uncomfortable. But when you have to go through with it, you just get through it. You joke your way out of it. I remember the first time it happened to me when I was first in Thailand. My friend took me to a Chinese wedding, and you know how a Chinese wedding is, there can be hundreds of people. And when you come in as a foreigner, you're something special. So then they called me on the stage and asked me if I could say a few words. And I thought 'shit' but then I joked my way out of it, saying some amusing thing. Later on I understand it really doesn't matter what you say on the stage, as long as you say something nice and then everybody is happy.
}

The next case of ZIA unveils intriguing cases of cultural chameleons, at both individual cultural unit and organizational levels - the key theme of the narrative in Chapter 7. The above passage serves as part of the evidence in a detailed analysis on cultural chameleon and its unique functioning.

\subsection{Cross-cultural intelligence (XCQ)}

The second core category of the proposed grounded theory is the heuristic-based capability needed for successful cross-cultural interaction, 'cross-cultural intelligence' or $X C Q$, so named to differentiate it from the more general 'cultural intelligence' or $C Q$. 
Most particularly, the concept of XCQ is an expansion of the CQ concept to stress its occurrence not simply during interactions in unfamiliar cultural contexts but in situations in which cultures specifically cross. Thus, XCQ constitutes the facility or power to exercise cross-cultural interaction to achieve desired and desirable results when actors operate in a cross-cultural interface. That is, XCQ is a learning-by-doing execution of the ability to practice cross-cultural heuristic effectively.

The general term intelligence is rooted in the field of human intelligence, which houses two major competing schools of thought: the general intelligence view versus the faceted intelligence perspective (Gardner, Kornhaber, and Wake 1996, Mackintosh 1998, Sternberg 1985, Sternberg 2000, Wolman 1985). The conventional model of intelligence is the foundation of the quantitative measurement of general intelligence popularly labelled IQ (from intelligence quotient). According to this model, intelligence consists of a set of general properties (the $g$-factors) that can be objectively measured using a standardised test, whose results signify an individual's standardized level of intelligence (the IQ). Thus, this view looks at intelligence as a basic knowledge stock whose property is measurable and quantifiable. Recent developments in intelligence theory, however, espouse a different view, one that leans more towards the notion that 'intelligence is as intelligence does' (Bar-On and Parker 2000, Gardner, Kornhaber, and Wake 1996, Kihlstrom and Cantor 2000, Sternberg 1985, Sternberg 1997, Wagner 2000). This emerging view of intelligence focuses more on the flow of knowledge - the actual exercise of the stock of knowledge. According to this view, intelligence comes in multiple forms and manifests itself in multiple fashions. It was actually this alternative perspective that gave rise to concepts and theories such as social intelligence, multiple intelligences, and emotional intelligence. Following a framework established by Sternberg (1986), Earley and Ang (2003: 25-58) elaborated in detail the above development in the field of intelligence study and provided a structured and thorough review on the different perspectives of intelligence.

Once again, as with the expanded concept of intricate cultural webs, rather than drawing solely on pioneering research on cultural intelligence (CQ) (most particularly, Ang and Van Dyne 2008, Ang, Van Dyne, Koh, Ng, Templer, Tay, and Chandrasekar 2007, Earley and Ang 2003, Group \& Organization Management 2006, Thomas 2006, Thomas and Inkson 2003, Thomas, Elron, Stahl, Ekelund, Ravlin, Cerdin, Poelmans, Brislin, Pekerti, Aycan, Maznevski, Au, and Lazarova 2008, Thomas, Stahl, Ravlin, Poelmans, Pekerti, Maznevski, Lazarova, Elron, Ekelund, Cerdin, Brislin, Aycan, and Au 2008), the conceptualization of XCQ emerged originally from integration and analysis of the grounded evidence from analyzing the cases and their meanings in this study. In fact, it was the emergence of this core category from evidence in the cases that led to a theoretical sampling of literature and the eventual identification of the said stream of research. Nonetheless, because cross-cultural intelligence is a core category of the proposed theory, a conscientious incorporation of earlier work on CQ into the theorizing process, as part of the use of literature as another source of evidence, served a major role in the entire process. Such incorporation afforded a certain level of confi- 
dence to the subsequent theorizing on XCQ. The resulting conceptualization of crosscultural intelligence emphasises its qualitative and highly interpretive nature, which, as explained above, is signified earlier by the addition of the letter $\mathrm{X}$ to the standard acronym for cultural intelligence, $\mathrm{CQ}$, to signal the important complementary distinction between the concept of XCQ and the more generally deductive and quantitative approach of CQ.

Fundamentally, XCQ is the strategic capability that a cultural unit needs to handle the intricate cultural webs of its own cultural identity while operating in the intricate cultural webs inherent in any cross-cultural interface. Thus, the locus of XCQ is not in the nature of its focussed entity - that is, intelligence about culture and its influences but in the nature of its interactive facet - that is, intelligence when operating in crosscultural situations. In contrast, $\mathrm{CQ}$ as initially explored by its most prominent pioneers was primarily, although certainly not exclusively, defined in terms of the ability to act effectively in an unfamiliar cultural context (Earley and Ang 2003: 59, Thomas and Inkson 2003: 62). Nevertheless, of late, $C Q$ research has paid more attention to the issues of cultural diversity and the multi-cultural context (Ang, Van Dyne, and Koh 2006, Ang, Van Dyne, Koh, Ng, Templer, Tay, and Chandrasekar 2007, Thomas 2006, Thomas, Elron, Stahl, Ekelund, Ravlin, Cerdin, Poelmans, Brislin, Pekerti, Aycan, Maznevski, Au, and Lazarova 2008, Thomas, Stahl, Ravlin, Poelmans, Pekerti, Maznevski, Lazarova, Elron, Ekelund, Cerdin, Brislin, Aycan, and Au 2008). Accordingly, the concept of crosscultural intelligence fully takes into account the ability to operate successfully in a context where the crossing of more than one culture is the salient condition. In doing so, it puts sole emphasis on the implications of the interaction (i.e. crossing) of cultural forces. The fundamental theoretical difference is one not of substance but of focus, as was also keenly observed by Thomas (2006). Based on this premise, I characterize cross-cultural intelligence as a sub-set of cultural intelligence or $C Q$, since $X C Q$ is certainly part of a cultural unit's ability to act effectively in one unique instance of unfamiliar cultural contexts, that in which cultures actually 'cross', in any of the four major manners theorized in the earlier section.

\section{Nature of XCQ}

An illuminating way to clarify the nature of XCQ is to compare it with the type of intelligence required in such tacit know-how as driving skill. In learning how to drive, a learner cannot actually get on the road only by reading or attending lectures on the theory of how to drive; he or she needs to actually take the driver's seat and start driving, no matter how clumsily. Likewise, reading a book or even attending cross-cultural training sessions can at best be only a prelude to exercising XCQ. An individual needs to actually be involved in an actual cross-cultural interaction to know how to exercise $X C Q$, however awkwardly at first. This analogy can be extended to many other aspects of both driving and XCQ, including assessment, mistakes, accidents, and experiences. Those who pass the theory test do not necessarily know how to drive; those who pass the road test do not instantly drive well. A driver's license only indicates an individual's 
potential ability to drive, whereas driving skill is a life-long practice. Only those who have been on the road long enough can drive adequately and only some all of them can drive proficiently. The same analysis can be applied to XCQ.

Thus, XCQ can be theorized to have three key properties: tacit knowledge or knowhow, embeddedness in practice, and situational dependence. Regarding the first property, Polanyi's landmark scrutiny of tacit knowledge (Polanyi 1974, Polanyi 1983) generated numerous inquiries into this particular type of knowledge and inspired burgeoning work on knowledge management and organizational learning which has also been augmented by research showing the significance of tacit knowledge as related to intelligence (Boisot 2002, Chiva and Alegre 2005, Cook and Brown 1999, Dierkes, Antal, Child, and Nonaka 2001, Fiol and Lyles 1985, den Hertog and Huizenga 2000, Mirvis and Mirvis 1996, Nonaka 1991, Nonaka 1994, Nonaka and Takeuchi 1995, Sternberg 1997, Wagner 2000). Again, relevant insights on tacit knowledge from this body of literature underpin the conceptualization of XCQ.

As tacit knowledge, XCQ is not a codifiable knowledge type and although some part of it can be coded (in the form of how-to books, for example), such codes, useful as they may be, are far from sufficient for a successful cultivation of XCQ. That is, XCQ must be embedded in practice, as the driving analogy illustrates. The apparent implication of this is that practice is not only imperative but, as the old adage claims, 'practice makes perfect'. Given that, mistakes and accidents are neither avoidable nor uncommon in XCQ practice and serve as part of the learning process.

Finally, the exercise of XCQ is very situational dependent in that it requires constant assessment and sensitivity to the actual situation or context. Hence, it is nondeterministic and must be based on a flexible heuristic rather than a rigid formulaic procedure. Moreover, since XCQ is manifested in its practice, its assessment is not completed until it is being evaluated during an actual action. In this sense, quantitative measurement of XCQ is analogous to assessing an individual in the theoretical part of the driver's test: it only confirms knowledge about traffic and signals possible driving ability. To assess how well that individual actually drives is a matter of taking a passenger's or examiner's seat and observing his action on real traffic. This attests to the value of qualitative approach in conceptualizing and understanding XCQ - a kind of $X C Q$ road test, so to speak, an issue to be revisited in the concluding chapter.

\section{3. $X C Q$ amid intricate cultural webs: a grounded theory}

The major concepts elaborated so far - culture, intricate cultural webs, cultural crossing and its major modes, and cross-cultural intelligence - form the building blocks of the core research outcome, the grounded theory of 'XCQ amid intricate cultural webs'. This theory, which explains how a cultural unit operates on a cross-cultural platform, could aid in both the analysis of cross-cultural interfaces and effort to manoeuvre such interfaces successfully. It is conceptually depicted in Diagram 6.2 and can be elaborated as follows. 


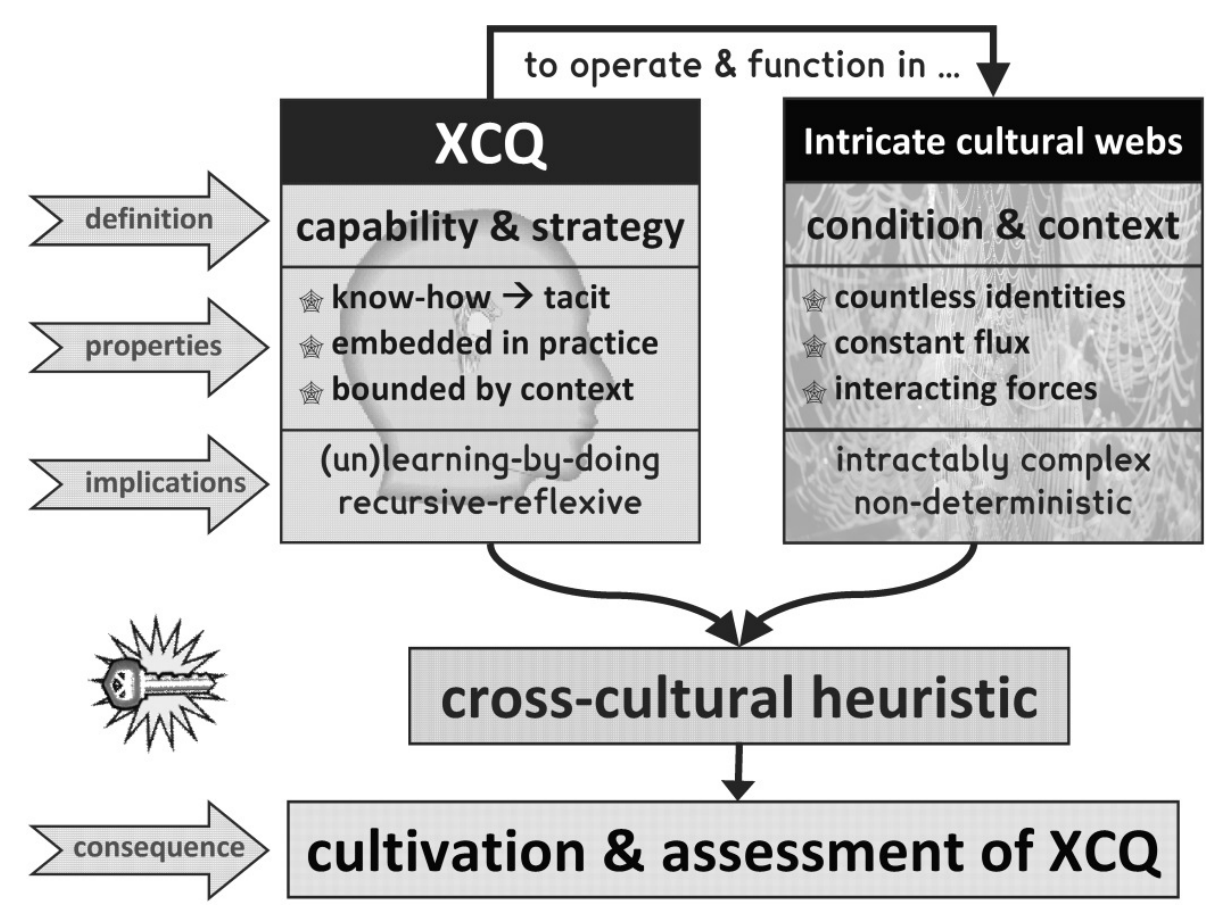

Note: Author's conceptualization based on study evidence.

The underlying condition of a cultural unit's XCQ is its cultural identity, which comprises multiple dynamic cultural webs. When a cultural unit operates in a cross-cultural condition, it must function in yet another set of intricate cultural webs. Cross-cultural condition, in this sense, comprises intricate cultural webs as its underlying property. This dynamic can in turn be extended to larger and larger networks of cultural webs. For example, take the case of Khun Pim of ADT, he himself was an individual cultural unit made up of intricate cultural webs (male, Dutch, engineer, top management, computer industry professional, Christian, husband and father, and so on). He was then employed to operate in the specific cross-cultural environment of a Dutch multinational company in Thailand, a cultural unit that was a group which had its own intricate cultural webs (Thai, Dutch, departments, cliques, employees of different social classes and so forth). The Thai subsidiary of this multinational was yet another cultural group functioning in one or more cross-cultural platforms with even more intricate cultural webs (including the large multinational firm it belonged to and the Thai cultural scene).

If this image sounds excessively complex, then I certainly make my point. Realworld cross-cultural conditions are intractably complex, comprising multiple meanings that are constantly in contention. To cope with such intractable, non-deterministic conditions and contexts, XCQ must have one important characteristic: it must be a 
recursive-reflexive ability and strategy. It must be recursive because of its (un)learningby-doing nature - individuals must call on their XCQ to exercise XCQ - and reflexive because the more individuals practice cross-cultural interaction, the greater their mastery of it and the more they cultivate their XCQ. In brief, having a recursive-reflexive property means that exercising $X C Q$ requires the use of $X C Q$, and that practicing $X C Q$ is itself crucial to the process of cultivating XCQ.

The key to a cultural unit's operation in the complex condition of intricate cultural webs is the cross-cultural heuristic - a simple strategy for dealing with complex situations, which lies at the heart of cross-cultural intelligence and is the topic of the very next section.

The above properties of XCQ amid intricate cultural webs have several implications for XCQ cultivation and assessment. First, the proposed theory suggests that XCQ is best developed by its constant practice while any formal education to cultivate XCQ, such as cross-cultural training courses, should employ an experiential learning approach, involving such tools as cases, simulations, games, or role-playing exercises. In the same vein, the proposed theory implies that any meaningful assessment of XCQ must involve seeing it in action. Thus, although not unappreciative of the current pioneering efforts to develop quantitative assessment and measurement instruments for cultural intelligence by the pioneering group of researchers (Ang, Van Dyne, and Koh 2006, Ang, Van Dyne, Koh, Ng, Templer, Tay, and Chandrasekar 2007, Ang, Van Dyne, $\mathrm{Ng}$, and Koh 2004, Earley and Mosakowski 2004, Thomas, Elron, Stahl, Ekelund, Ravlin, Cerdin, Poelmans, Brislin, Pekerti, Aycan, Maznevski, Au, and Lazarova 2008, Thomas, Stahl, Ravlin, Poelmans, Pekerti, Maznevski, Lazarova, Elron, Ekelund, Cerdin, Brislin, Aycan, and Au 2008), I argue here and elaborate further in the concluding chapter that developing a set of complementary qualitative assessment tools based on real-world observation and experiential philosophy is crucial to a thorough assessment of XCQ in particular and $C Q$ in general.

One final vital point is XCQ's relation to the issue of level of analysis. Essentially, the concept of XCQ is applicable to any type of cultural unit - either an individual or groups (whether an organization, nation, global, family, clique, and so on). Fundamentally, every cultural unit (individual or group) must possess a certain level of XCQ in order for it to function adequately at a minimum. First of all, every cultural unit is itself multicultural, having to deal first and foremost with the intricate cultural webs that characterize its very own identity. Moreover, since, from the view of culture informed by this theory as elaborated in Chapter 4 , social conditions that involve a single culture are practically non-existent, every cultural unit must invariably operate on at least one, but most likely many, multicultural platforms. Thus, a cultural unit, no matter individual or group, needs to exercise XCQ to handle both its own identity and its environment. In principle, we can thus speak of XCQ of an individual, a team, an organization, a nation, and all other types of cultural groups.

As a matter of fact, the theory of XCQ amid intricate cultural webs compels, rather than restricts, simultaneous multi-level analysis in order to exploit its full analytical 
power and render insightful understanding of real-world cross-cultural phenomenon. Further theorization and refinement of the XCQ concept and the effective analytical approach vis-à-vis levels of analysis, both in breadth (investigating how the numerous levels are associated) and in depth (examining each possible level of analysis or cultural group in detail) is an attractive candidate for future research.

\subsection{Cross-cultural heuristic}

Cross-cultural intelligence, as a capability and strategy, can only be manifested and witnessed 'in action'. XCQ in action, in turn, is best captured and observed under a schema of a heuristic procedure that lies at the heart of cross-cultural intelligence. The theorized procedure is termed simply 'cross-cultural heuristic' - the very subject of this section.

The term heuristic is selected for this theoretical concept under the influence of its use and meaning in the disciplines of management science/operation research and computer science (Michalewicz and Fogel 2000, Pólya 1973) because the gist of XCQ as revealed in the grounded evidence has the properties that correspond to a heuristic procedure pioneered in the two disciplines and now explored further in social sciences (Gigerenzer, Todd, and the ABC Research Group 1999). ${ }^{28}$ A discussion on the properties and meaning of heuristic begins this section, followed by a presentation of the details of cross-cultural heuristic that emerged from the evidence in this study.

Heuristic, in management science/operation research and computer science, is a procedure to reach a solution to a problem that serves an alternative approach to algorithm (Michalewicz and Fogel 2000, Pólya 1973). The sub-field of 'theory of computation' provides a clear taxonomy of classes of problems and ways to solve them (Lewis and Papadimitriou 1998). According to this taxonomy, there exist two major classes of problems: 1) simple, thoroughly solvable problems for which definite algorithms can be derived to solve for guaranteed solutions, and 2) complex problems for which no algorithm can be formulated to solve for guaranteed solutions. For deterministic, thoroughly solvable problems or situations, a finite step by step instruction can be devised that would require manageable resources to apply and solve for proven solutions. Such a procedure is an algorithm. However, only a certain class of problems or situations are simple enough to lend themselves to clear-cut algorithmic procedures (ones that require manageable resources and involves finite steps) with proven results. A large number of cases, unfortunately, fall in the second category of complex problems for which proven algorithms to deal with them are either not possible (unsolvable problems) or not practical (intractable problems). Complex problems, thus, lend themselves to a different type of simple routines that could lead to at best 'satisficing' solutions. Such is a heuristic procedure.

\footnotetext{
${ }^{28}$ Reflexively, my previous training in management science/operation research and computer science has definitely influenced why the term emerged to denote this concept.
} 
Computational literature on solving complex problems identified the following distinct characteristics of complex situations or problems (Dörner 1997: 37-41, Funke 1991: 186-187, Michalewicz and Fogel 2000: 11-30): 1) multiplicity, i.e. involving large, often overlapping and conflicting, numbers of factors, parameters, and goals; 2) complexity, i.e. involving infinite interrelation among factors and parameters; 3) dynamic development, i.e. parameters and properties are invariably changing and influencing each others, such that the assumption of ceteris paribus can never hold and the principle of 'divide and conquer' is rendered inapplicable; and 4) opacity or intransparency, i.e. factors and relationship are not easily discerned or determined. For such a problem or situation that is 'difficult', an algorithm is usually either not possible or derivable and hence the solution is inevitably non-deterministic.

In the field of human intelligence in general and social intelligence in particular, recognition of the complexity of real-world situations that defy simple deterministic problem solving process is well documented. Following Neisser's discussion of the difference between academic and practical intelligence (Neisser 1976), Earley and Ang's characterization of Neisser's distinction was very much in line with the taxonomy of problem as established in computational literature.

[...] Neisser (1976) reasoned that individuals who perform well in academic settings may not necessarily thrive in real-world settings because the problems faced in academic settings are very different from those of the real world. In school, students are often required to solve problems that are (1) well defined, (2) already formulated by others, (3) come complete in the information required to solve the problem, typically only has one correct answer, and one correct method of getting the right answer, and (4) are simplified so that they are out of the context of the ordinary experiences of similar problems in the real world.

In contrast, the practical everyday problems faced by individuals in nonacademic settings tend to be unformulated, or in need of formulation, or reframing. Individuals work with incomplete information and have to deal with dilemmas of multiple correct solutions, all only satisfying at best, with none necessarily optimal. Practical problems are also characterized by their many different ways of arriving at these solutions.

(Earley and Ang 2003: 50-51)

Earley and Ang (2003) then related how this distinction shed lights on the functioning of Steinberg's practical intelligence (Sternberg and Grigorenko 2006), the type of intelligence needed to solve real-world problems, of which CQ (which includes XCQ) is arguably a subset. Most real-world social situations belong to the class of intractable problems at best, unsolvable at worst. Cross-cultural interaction is certainly a very difficult social situation, since the very nature of intricate cultural webs elaborated in Chapter 4 does indeed manifest all of the four characteristics of a difficult situation or complex problem highlighted above. As with complex computational problems, an ambition to come up with a comprehensive formula, or algorithm, to untangle the whole 'intricate cultural webs' is a Herculean task which, even if successful, would end up delivering a model or algorithmic procedure that is so complex it will prove to be either impractical to apply at best, impossible at worst.

Recently, an interest in viewing human's problem solving as a primarily heuristic process has emerged. With the recognition that real-world situations are 'shrouded in a mist of dim uncertainty' where people 'have only limited time, knowledge, and com- 
putational capacities with which to make inferences about what happens in the enigmatic places in their world' and must make use of 'fast and frugal heuristics' (Gigerenzer, Todd, and the ABC Research Group 1999: vii), the authors of Simple heuristics that make us smart set out to explore the many simple heuristic routines people employ in their dealing with a wide variety of everyday problems. The authors observed in their conclusion that heuristics employed in social interaction domain 'is one of the most important areas still to be mapped out' (Gigerenzer, Todd, and the ABC Research Group 1999: 363-364).

The conceptualization of cross-cultural heuristic could contribute to this 'most important' domain of research in social heuristics that 'make us smart'. In real-world situations where individuals have no choice but to deal with complex situations in the tangle of intricate cultural webs, most develop the capacity to deal with them in a simple, yet elegant manner - the heuristic route. The actors in the three narratives appeared to follow a certain kind of heuristic in wading their ways through intricate cultural webs. Their individual success in doing so might vary in degree, but one thing was certain, most did attempt to deal with the situations in a heuristic manner, however complex and difficult those situations might be. Following is a theorization of the heuristic procedure these actors exercised in the case evidence and concurred by relevant literature.

\section{Steps in cross-cultural heuristics}

Diagram 6.3 outlines the four step procedure in cross-cultural heuristic. This section elaborates on each of the steps and how they are related to one another in the practice of XCQ.

\section{Invoke negative capability to form an open mind}

Step one in cross-cultural heuristic is to invoke negative capability to form an open mind. This may sound like a fancy cliché, yet it is the most difficult step to take a full command of and the one element of CQ unanimously identified by all major theorists (Ang and Van Dyne 2008, Ang, Van Dyne, and Koh 2006, Ang, Van Dyne, Koh, Ng, Templer, Tay, and Chandrasekar 2007, Sternberg and Grigorenko 2006, Thomas 2006, Thomas, Elron, Stahl, Ekelund, Ravlin, Cerdin, Poelmans, Brislin, Pekerti, Aycan, Maznevski, Au, and Lazarova 2008, Thomas, Stahl, Ravlin, Poelmans, Pekerti, Maznevski, Lazarova, Elron, Ekelund, Cerdin, Brislin, Aycan, and Au 2008, Triandis 2006).

The narrative of ZIA in the next chapter will illustrate a key concept which I decided to name using Keat's terminology - negative capability. Negative capability refers to an ability to achieve a state of an open mind that creates a greater chance of forming a robust understanding of the unfamiliar and the unknown. Since its first appearance, this term and its meaning has influenced much theorizing, not only in the literary circle, but also in other fields, including psychology and management, where negative capability is theorized to be an essential characteristic of effective leaders and a key 
ingredient for management under uncertainty and change (French 2000, French 2001, Simpson, French, and Harvey 2002). There are numerous interpretations of Keats' original concept, but the one that is most in tune with the meaning of the term as used here in relation to cross-cultural heuristic was by Genoways who described negative capability as a 'spirit of rigorous open-mindedness, [a] willingness to view any issue critically but from all sides' (Genoways 2005: 2). Not to reinvent the wheel, I settle for Genoways' definition to be the definition of the term negative capability for the proposed cross-cultural heuristic.

\section{Diagram 6.3: Cross-cultural heuristic}

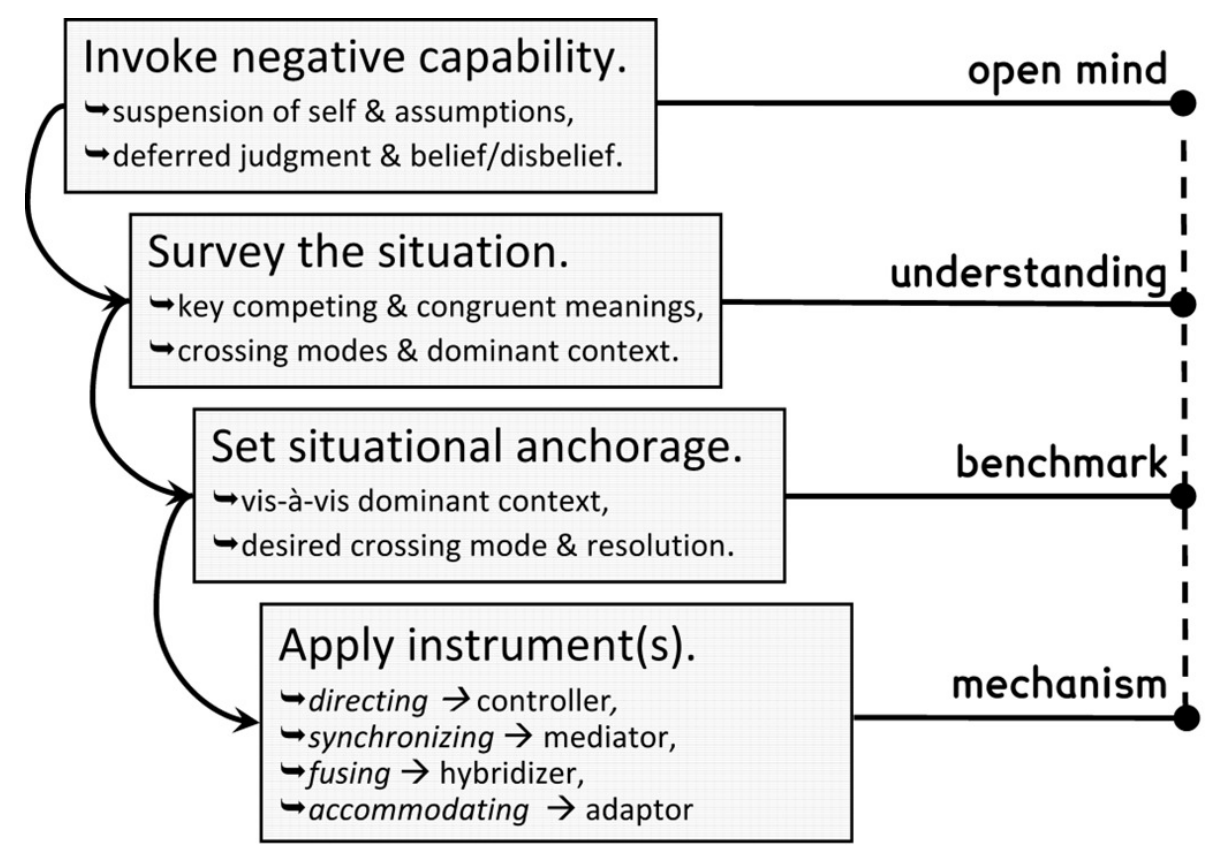

Note: Author's conceptualization based on study evidence.

The significance of negative capability in cross-cultural heuristic lies in the fact that it designates the very first step in dealing with and functioning in a cross-cultural condition. Cross-cultural condition is intractably complex, marked by a strong presence of the unfamiliar and the unknown that confront a cultural unit with great 'uncertainties, Mysteries, doubts' (Keats and Forman 2004: 57). In such circumstances, basic human instinct often dictates a need to apply what is known to the unknown, to quickly reach a rational conclusion in order to ease the unease created by the unfamiliarity (French 2001). However, such quick and fast 'reaching after fact \& reason' (Keats and Forman 2004: 57) does not always yield rigorous understanding. Khun Pim of ADT gave a vivid account of such tendency when he described how he initially suspected the readiness to please in the Thai people when he first encountered them. In his words, he stated, 
Let me explain. When I first arrived in Thailand, in Bangkok, in the beginning, I was suspicious. I hope you understand what I mean. The behaviour was extremely friendly, so willing to help. So I thought, what are they thinking, are they looking for something. But later on I learned this is true. This is really the way they are. They like to give a lot of things to other people, and then maybe later, in another way, they may get good things back. I always tell other people, it's a kind of investment. They invest by doing good things for other people so they may get good things back later, in another way. Then I started to accept it. But in the beginning I was suspicious. I entered Bangkok as a foreigner and then I had people coming to offer helps. Do you want a taxi? Are you looking for a hotel? And so on. And so I was overwhelmed and thought, they were looking for money, for sure.

Khun Pim, as an individual cultural unit, illustrated a movement from instinctive 'reaching after fact \& reason' to a 'negative' and richer understanding of a common Thai trait: the willingness to please. At first, he simply applied a usual Dutch interpretation of this gesture - people offer services in return for direct remuneration - an interpretation that reflects a common Dutch view of exchange: trade-mindedness, egalitarianism, and a straightforward system of reward. His suspicion was an unease that resulted from the conflicting message between the gesture and the meaning he applied to it. The following questions must be running through his mind: they acted like they wanted money, but then why didn't they state the price upfront; why did they have to be so friendly about it; what did they really want? Once his mind was open and his Dutch inclination was suspended or 'negated', however, he discovered other possible meanings of such friendly gestures that are more in sync with common Thai view of exchange: the notion of karma, indirect and long-term expectation, and a subtle system of reward. Interestingly, Khun Pim still used a very Western term to denote the alternative meaning he just deciphered - the term 'investment' - while his description of it was actually more in line with the Thai meaning of karma, when he stated, 'They like to give a lot of things to other people, and then maybe later, in another way, they may get good things back'.

Negative capability inherently demands a pre-condition of first suspension of self and assumptions, and second deferred judgment. In a sense, the need for negative capability suggests that in any cross-cultural interaction, a certain degree of 'unDutching' is called for: a need to temporarily disassociate from one's cultural identity to an extent that the assumptions and judgment that come with it exert no influence during the search for meanings. Suspension of self and assumptions is thus a chameleon like ability, as will be further discussed at the end of the ZIA narrative. The chameleon analogy highlights a fine distinction between suspension and elimination. Suspension of self and judgement does not equate with elimination of either; it is only a transient state that allows one to make room for other 'selves' and other 'assumptions' in such a way that one can form an empathy with them - the unknown becomes the known, the unfamiliar now familiar. The empathy is next reinforced by deferred judgment and belief (or disbelief) because any judgment at the point of a search for meanings will not only prevent one from embracing new findings but also blind one from looking any further. If Khun Pim had settled for the interpretation that the friendly Thais were only after money, he would never have discovered alternative meanings for their friendly 
gesture. Judgment in whatever direction - right or wrong, true or false, real or unreal, and so on - blinds the mind from more insights and puts a halt in any search.

Negative capability functions as a heuristic practice because it does not seek to guarantee that one would get 'the' true meaning of anything, it merely opens up one's eyes to as many possibilities of meanings as may exist, a condition inherent in any cross-cultural interface: myriad meanings in contention. Hence, the more meanings one can identify, the better; while a quest for the one 'true' or 'right' meaning is simply meaningless and would in fact stunt the search for meanings. This is what is meant by robust understanding - an understanding that is strong and healthy because of its open-mindedness. In the above example from Khun Pim, it can be argued either way that the friendly Thais in this case were indeed after quick monetary gain, or that they were simply being friendly because they believed that would bring good karma to their lives. The point is, it does not really matter at this step what the right argument is, because with negative capability, the argument or meaning any individual subscribes to is always the right meaning to that individual. What is important, however, is the ability to see what the myriad 'right' meanings are there in contention. The eventual 'right' meaning will be a relative one, a task of a later step in cross-cultural heuristic. In the very beginning, however, without a thorough search for as many relevant meanings as possible, the identification of a 'right' meaning for a given situation will be not as effective. Negative capability ensures that such a search is as thorough as it can humanly be.

Negative capability is, to repeat Genoways's definition (2005) '[a] willingness to view any issue critically but from all sides.' There is a fine line between being critical and being judgmental. Critical examination is inclusive whereas judgemental assessment exclusive. At the end of the first step in cross-cultural heuristic, what is achieved should be a critical but open mind that is ready to identify and include as many competing and congruent views of a situation as possible - the exact task of the next step in this heuristic procedure.

\section{Survey the situation to form an understanding}

Step two in cross-cultural heuristic is to survey the situation to form a robust understanding of the pertinent state of affairs. Two aspects of the situation to be surveyed are the key meanings and its context.

What need to be surveyed first and foremost are the key competing and congruent meanings. The complexity of intricate cultural webs would present a cultural unit with countless issues, each with myriad meanings, some conflicting whereas others congruent. In order not to be ensnared in the cultural webs, a cultural unit needs to find a focus by first identifying what the key issues are and then concentrating the search for only the meanings of those key issues.

Key competing and congruent meanings refer to those meanings that are either starkly differing (competing) or highly compatible (congruent) and will hence most likely exert significant force in the crossing of cultures. Specifically, the term 'significant 
force' denotes only the issue of strength and, following the spirit of negative capability and open-mindedness, does not carry with it any judgmental valuation. It means simply that the identified sets of competing and congruent meanings have a great potential to define the nature of the crossing of the cultures in questions. It does not, in any way, indicate the exact direction the crossing situation will develop. Opposites can attract as well as repel, whereas similarity can charm as well as bore. Again, under the spirit of the cross-cultural heuristic, any direction of a cross-cultural condition is highly situational and is hence not predictable by any static formulaic manner. It unfolds only when put 'in action'.

The case of 'three strike and you're out' at ADT, as a cultural unit that is at a group level (that is the group of Dutch and Thai employees interacting to serve a business organization) can be used to illustrate. First, this episode was set in a context that involved numerous issues: quality control, communication channel, corporate policy, customer service, marketing strategy, and so on so forth. However, the focus of the crisis could be identified as the competing meanings of 'mistake' held by the Dutch and the Thai, which resulted in how the episode was interpreted differently by the various characters, eventually leading to a tension. In this situation, it would pay for the group to survey how the different people involved looked at the notion of mistake, what it meant to them and how those meanings had influenced their actions, thoughts and feelings. Similarly, in the narrative of the next case ZIA, competing meanings of Dutch v. Thai views of project management also provide a similar illustration for an existence and search for key competing meanings. In contrast, CDW offers the other side of the coin - an existence of congruent meanings, where different views of the same issue, such as professionalism or rank, complement one another and could be a basis for a combination or fusion.

Once key issue and its competing and congruent meanings are surveyed, the next task in this second step is to identify initial crossing mode and the dominant context of the situation at hand. Again, as noted earlier regarding mode of crossing in crosscultural context, there may be more than one mode co-existing in one particular situation. At this point in the heuristic, the task is to identify the initial crossing mode, that is, the one that dominates the situation as it begins to unveil. The nature of the initial crossing mode is important because it will provide needed information for the third step, where desired crossing mode and steady state resolution is identified. Without knowing what currently transpires, a movement towards what is desired will be rather haphazard rather than purposeful.

To carry on with the same examples, the crisis episode of mistake at ADT clearly had a clashing condition as its initial crossing mode. True, there were other crossing modes in the case. Khun Pim's affinity towards his Thai employees and the Thai culture and Khun Maleewan's warm feeling for ADT and her Dutch bosses were more of a unification mode; while Khun Kees's resignation that 'if I can get the products in at the right time, with the right price, and of the right quality, whatever happens there, it doesn't matter' revealed more of a reciprocal condition. Yet, for the 'mistake' episode, 
a clash was dominant. The situation was characterized by a strong tension as a result of how the various actors view the situation and its relation to the concept of 'mistake' differently. The decree of 'three strike and you're out' was an exercise of control on the Dutch executives' part. At the same time, the Thai factory had previously exercised a different source of control: the control that came from being local, which meant they were the closest to the situation and allowed them to be the first to respond. Up to this point in the episode, the state of affair was of a division, each party exercising their directing instrument to take control of the situation as they saw fit. Similarly, section 7.3 in Chapter 7, on variant cross-cultural modes identifies several co-existing cultural crossing modes at ZIA with one crucial clashing mode regarding the concept of project management as the most poignant. Applying the same analytical scheme to the CDW case will also yield similar insights and reveal an initial crossing mode as being a mix of two co-existing modes: reciprocal and unification.

After identifying the initial crossing mode, one more important survey needs to be done before the next step in the cross-cultural heuristic can start: a survey to identify the dominant context of the situation. By dominant is meant the context that defines the significance of the whole situation, one whose meaning is critical for the situation to be of importance. In all narratives in this study, the dominant context was simply the 'bottom line' of the business. In a nutshell, the business context was pre-eminent in the cases here, as the unit of analysis is indeed that of a business firm and its operating unit. No doubt, other contexts might be of influence, but they were not the one that defined the situation in all three cases and two vignettes in this study. The social relationship among the actors, the networking context between the Thai and the local customers, for example, might be of influence to some of the actors but these were not the context that defined the situation that brought these actors together, and must continue to define their inter-relationship.

Naturally, in cross-cultural platforms outside of a business setting, dominant contexts of other nature abound. For example, the dominant context of a cross-cultural family unit is most likely a constancy of family life, whereas at a global level, we could argue that continuity of the human race defines global cross-cultural landscape. Note, though, that this identification of dominant context is distinct from desired steadystate resolution. Both constancy of family life and continuity of the human race can be attained by various means. A family in which one partner completely dominated the other could survive just as well as that in which both partners are completely in unison. The same is true regarding continuity of the human race, which, theoretically if not appealingly, could come about through war as well as peace.

Once key competing and congruent meanings and the initial crossing mode and dominant context are defined, it is time to move on to the next step where the desired steady state resolution is assessed, so that it could be commanded by the exercise of cross-cultural heuristic and not left completely to chance. 


\section{Set situational anchorage to establish a benchmark}

Step three in cross-cultural heuristic is to set situational anchorage to have a benchmark for desired crossing mode as a steady state resolution. The key word here is 'situational', because it is the situation as defined in the second step that serves as the anchor to set an effective benchmark.

This step in cross-cultural heuristic resonates with a recent observation made by a number of prominent cultural researchers - a plea for greater and closer attention to the significance of context in cross-cultural analysis (Boyacigiller, Kleinberg, Phillips, and Sackmann 2004, Earley 2006, Earley and Singh 1995, Jackson and Aycan 2001, Peterson 2006, Smith 2001, Triandis 2001, Yeganeh, Su, and Chrysostome 2004). Based on this study's evidence, cultural units indeed rely heavily on situation as a guidance for deciding which one of the competing meanings should be the 'desired' one. Simply put, the 'desired' meaning and resolution are the ones that serve the context best.

To continue with the same illustration used in step two, the group at ADT in fact had several options to select as their desired crossing mode and resolution. Since the dominant context was the business of ADT, the resolution must be one that served this context well. Given the fact that ADT was predominantly a Dutch owned and managed company, the most efficient way was to preserve management philosophy and practice that had served the company well and was being exercised throughout the rest of the company. In this case, it was the policy of keeping a single communication point vis-à-vis external parties. The question then became: how this benchmark could be reached. This was where several options presented themselves.

Let us examine first the option that seemed to have been selected as far as the investigation for this study uncovered. At the end of the case, one option had apparently been selected: the 'three strike and you are out' option. Khun Kees the CEO was adamant that if such 'mistakes' were to happen again, somebody would have to bear the consequence of it, because the policy must be upheld. That was the key message in the letter from the CEO. The resolution in this case, on the Dutch executives' part, was simply to uphold the clashing condition and bring it to either an assimilation or separation steady state. Which of the two it turned out to be would depend on how the party at the Thai plant reacted. If the Thai understood or accepted the 'meaning' of the situation as decreed by the Dutch, then assimilation would be the resolution. They would carry on their function and replace the new notion of 'mistake' that was different from what they would see in other situations outside of this context. However, if the Thai insisted on their interpretation of the situation, those who did so would likely have to separate from the company altogether.

ZIA, on the other hand, provides a different desired steady state resolution to a clashing initial condition concerning competing views of Dutch v. Thai project management. As analyzed in the next chapter, ZIA management preferred instead to move from a clashing to variation cross-cultural condition, and hence exercised their chameleon like ability to engineer a chameleon steady state resolution, where the Thai engineers were lured into applying Dutch instead of Thai project management principles. 
CDW, by contrast, appeared to discern that the initial reciprocal crossing mode was exactly what the firm needed and hence continue to further maintain and enhance it into an effective aggregate resolution, as already elaborated in the narrative.

Khun Kees of ADT reflected vividly how this third step in cross-cultural heuristic was applied in a simple yet elegant manner, on the ground:

I personally believe that the relationship must be good but it's the result that counts. If the good relationship helps to get good results then that's good but it's not always the case. It could sometimes be a bad thing to have a good relationship. Every company is set up to make a profit, so it's the result that must take priority, not the relationship. The relationship must not be above the result. You do not build a company to have a good relationship with the people who work for you. That's not what a company's for.

\section{Apply instrument as the mechanism to reach desired resolution}

Once a benchmark is set, the fourth and final step in cross-cultural heuristic is to apply appropriate instrument as the mechanism to reach desired resolution. Four primary classes of key cross-cultural instrument are as introduced in earlier discussion on the taxonomy of cross-cultural condition: controller, mediator, hybridizer and adaptor (see Diagram 6.1).

Cross-cultural instruments come in many shapes and forms, ranging from formalized ones such as rank, authority, legality, financial means, and so on, to incidental ones such as power, knowledge, network, locality, charm, manipulation, and so forth. Some instruments take a concrete form, such as weapons or dictionaries, whereas others are more abstract such as affinity or respect. In fact, the varieties of crosscultural instrument are endless and only limited by the resourcefulness of a cultural unit. Whatever shapes and forms they take, the one characteristic all instances of cross-cultural instrument share is that they can be used to achieve a desired function: that is, to either direct (controller), synchronize (mediator), fuse (hybridizer) or accommodate (adaptor) - as summarized in Diagram 6.3. Although one particular form of instruments may lend itself better towards a particular function, any single instance of cross-cultural instruments can, theoretically, be exercised to achieve any of the four possible functions. Authority, for example, is a natural instrument to exercise control, while charm can be used to act equally well as a controller, mediator, hybridizer, or adaptor. Thus, what is crucial is to understand how these various instances of crosscultural instrument are exercised and to serve what function. Also, although each of the four classes of key cross-cultural instrument is best suited to different crosscultural modes as indicated in Diagram 6.1, all four can be exercised simultaneously in any cross-cultural context, depending on what particular resolution is desired at a particular point in the interface. The nature and properties of each of the four classes of cross-cultural instrument are as follows.

Controller refers to any instrument that renders those who exercise it a control over a situation such that they can direct its development according to their will. As long as what is exercised yields controlling ability to those exercising it, it is a controller. Controlling ability is the ability of one party to dictate the will, action, thought or feeling of other parties, fundamentally against their original wishes. The case of ADT 
displayed how controller could be used. To recall, Khun Kees's 'three strike and you're out' warning letter was a clear exercise of authoritative controlling power, whereas the controller of the Thai employees at the plant came in the form of their locality and network. Controller is perhaps the easiest class of instrument to discern as its effects are often vivid and obvious. The many violent means of settling differences that abound in our present day world affair provide ample evidence of the exercise of cross-cultural controller. Controller is the most effective instrument to sustain a clashing cross-cultural mode as it could efficiently lead to a steady state resolution of assimilation, division or separation.

Mediator refers to any instrument that acts as a 'go between' for difference parties in order to render a synchronizing effect. The need for a 'go between' comes basically from fundamental differences that call for a certain level of synchronization before the parties can interact with a tolerable level of friction, incompatibility or detrimental side-effect. The three primary roles that a mediator performs are as a bumper, connector, or interpreter. Bumper is the crudest and most basic function of mediator and serves a simple purpose of collision control through sheer diversion or absorption. Mediator when acting as a bumper simply absorbs or diverts potential frictions that result from differences. Khun Maleewan of ADT, for example, explained that one of her functions was simply to serve as a 'cultural bumper'. This was, for instance, when she described how she would listen to each of the parties, Dutch executives and Thai workers, and either blocked, screened out, or softened potential harmful meanings from those who might be hurt by them. Acting as a connector is the second role a mediator can perform. As a connector, a mediator serves by providing a channel for exchange. The need for a connector arises from any sort of distances or gaps - either conceptual or physical - that exist among different parties. In the case of ZIA, for example, Khun Fons described one particular instance of a connecting mediator, again in a form of an individual, as follows:

You can also try to have a representative of your employees, who can try to be a middleman in negotiating on serious matter. For example, if I have one of my engineers who are unhappy with something, he can then go to talk to the middleman, tell him about it. The middleman will then come to me and tell me about it. Then I'll think about it, find a solution and tell the middleman and he can bring the message back to the unhappy engineer. We can see who can be a good middleman after years of experience. We have a number of middlemen here in our history of the company.

The above description of a middleman illustrates how he or she serves as a connecting instrument by providing a channel for communication between the two different parties. The final primary role of a mediator is as an interpreter. As the name implies, this mediating function serves to translate meanings in one culture to their equivalent in another culture. The simplest example of an interpreter is of course a dictionary and the most sophisticated example of an interpreter is arguably a cultural theorist and his or her findings. In all its three primary roles, a mediator functions best to nurture a reciprocal state, as it acts to foster an aggregation of diverse cultural elements without injecting fundamental alteration to their core properties. At CDW, where steady-state cross-cultural mode is that of a reciprocal, we thus witnessed many instances of a me- 
diator particularly in the form of an individual such as Khun Chaowalit and Khun Busara.

The third class of cross-cultural instrument is hybridizer which serves the function of fusing or blending competing meanings to form a new hybrid meaning that have desired elements from its various sources. As with other instruments, hybridizer comes in many shapes and forms, from an abstract attribute, such as affinity and respect, that tends to instil a desire for unification, to an instinctive motive, such as survival instinct, that injects a need for a new hybrid cultural profile that would fit the situation at hand better than any existing identity. Hybridization is usually a long and complex process that is hard to observe meticulously. From this study evidence, we witnessed a cultural hybrid most vividly only at the level of an individual cultural unit, such as Khun Pim of ADT, Khun Geert of CDW and Khun Danny of ZIA. From these examples of a cultural hybrid, it appeared that initial affinity and prolonged experience with elements of other cultures acted as the hybridizer for both actors. Because of the obscured nature of hybridizer, especially at a group level, this is a research area very much in need of further study.

Finally, adaptor refers to a cross-cultural instrument that drives an adaptive response to help actors accommodate variation in cultural forces, either with regards to themselves as individual cultural units or in relation to aspects of the cross-cultural condition they are operating in. Let us take an electrical adaptor as an apt analogy to help clarify the function of a cultural adaptor. An electrical adaptor is a device that works to adapt an attribute of an electrical instrument in one system to enable it to function in a system other than that for which it is originally designed to work. The degree of differences in the two systems dictates what attribute(s) would need such adaptation, which in turn defines how sophisticated the adapting mechanism must be. At the simplest level where the two systems do not differ greatly - such as between the continental European and British electrical systems - an electrical adaptor only needs to adapt simple physical attributes: mainly the shape and wiring of the connecting plug to fit a different socket type. For systems of deeper discrepancy, such as the different voltages between the European and the American electrical systems, a more sophisticated adapting mechanism is required, involving transformation at the level of the electrical current. The same analysis may be derived in a cultural adaptor.

At a superficial level, for example, most foreigners try to learn and mimic outward gestures acceptable in an unfamiliar culture they venture into. The Dutch informants in this study, for instance, all related how they know that the Thai 'wai' was the equivalent of the Western handshake, and all would use the Thai form of addressing people with the title 'Khun'. Most would go on to mimic these outward gestures to indicate their willingness to blend in (an adaptive response) or accommodate the new cultural environment whenever they saw the need for such adaptive response. At a more sophisticated level, cultural adaptor works in a less discerning manner. The case of ZIA as analyzed in the next chapter provides numerous incidents when adaptor in various forms and levels of sophistication were exercised to create a chameleon like effect. 


\section{Notes on cross-cultural heuristic}

The followings must be noted with regards to the nature of cross-cultural heuristic. First, cross-cultural heuristic is an iterative and concurrent procedure. As Diagram 6.3 depicts, procedural steps in cross-cultural heuristic have a certain linear element in them but are by no means linear in actual execution. The arrows on the left in the diagram indicate a natural flow from one step to the next; yet the broken-line on the right indicates that such linear steps could be in concurrent with one another, and actors may take a step back as well as forward iteratively during the entire execution. As such, cross-cultural heuristic is best practiced with a flexible, situational spirit - completely in line with the property of XCQ as a tacit know-how that is embedded in practice and bounded by context as already discussed. Finally, as a heuristic, not algorithmic, procedure, cross-cultural heuristic does not guarantee optimal result or success; rather, it offers an intuitive, 'fast and frugal' means to reach a satisficing outcome or options of outcome, which vary across situations and actors. Cross-cultural heuristic is, to conclude, 'XCQ in action'.

\subsection{XCQ within the $C Q$ context}

The proposed grounded theory of cross-cultural intelligence or XCQ can be located within the overall effort of the emerging group of $\mathrm{CQ}$ theorists under a comparative review of how the variant perspectives of $\mathrm{CQ}$, including this XCQ concept, relate to one another in the overall picture.

\section{Variant perspectives of CQ: mutuality and complementarity}

To date, only a handful of researchers have rigorously investigated the theme of cultural intelligence as the core of their inquiry. Undoubtedly, the two most prominent voices are those of Earley, Ang, and colleagues (Ang and Van Dyne 2008, Ang, Van Dyne, and Koh 2006, Ang, Van Dyne, Koh, Ng, Templer, Tay, and Chandrasekar 2007, Ang, Van Dyne, Ng, and Koh 2004, Earley and Ang 2003) and Thomas and his team (Thomas 2006, Thomas and Inkson 2003, Thomas, Elron, Stahl, Ekelund, Ravlin, Cerdin, Poelmans, Brislin, Pekerti, Aycan, Maznevski, Au, and Lazarova 2008, Thomas, Stahl, Ravlin, Poelmans, Pekerti, Maznevski, Lazarova, Elron, Ekelund, Cerdin, Brislin, Aycan, and $A u$ 2008). Less visible, but as fervent in their focus on the centrality of $C Q$, are those who lean more towards the construct's practical and managerial implications (such as Peterson 2004, Plum 2007). The proposed grounded theory of XCQ amid intricate cultural webs adds yet another variant to this emergent stream of research.

Although the diverse perspectives on cultural intelligence vary on many fine points, they all share fundamental elements. Moreover, the variations among them constitute a complementary multiplicity of viewpoints and foci more than irreconcilable discrepancies. Diagram 6.4 summarizes this mutuality and complementarity of the variants in $\mathrm{CQ}$ research along the three dimensions of conceptualization, methodology, and implication which are highlighted in the subsequent discussion. 
Diagram 6.4: CQ: mutuality and complementarity in the variant perspectives

\begin{tabular}{|c|c|c|}
\hline Dimension & $\begin{array}{c}\text { Elements Of mutuality } \\
\sim \text { uniformity of the fundamentals }\end{array}$ & $\begin{array}{l}\text { Elements of complementarity } \\
\sim \text { diversity of viewpoints and foci }\end{array}$ \\
\hline Conceptualization & $\begin{array}{l}\odot \text { theoretical validity and practical } \\
\text { value of CQ } \\
\odot \text { complexity and significance of } \\
\text { culture } \\
\odot \text { multi-faceted view of intelli- } \\
\text { gence } \\
\odot \text { fundamental characteristics of } \\
\text { CQ } \\
\rightarrow \text { multi-dimensional } \\
\rightarrow \text { importance of behavioural } \\
\quad \text { and interactional aspect } \\
\rightarrow \text { tacit nature } \\
\rightarrow \text { critical success factor: open- } \\
\quad \text { ness to experience / mindful- } \\
\text { ness / negative capability }\end{array}$ & $\begin{array}{l}\text { diverse disciplinary back- } \\
\text { grounds: industrial/ organiza- } \\
\text { tional psychology, international } \\
\text { (cross-cultural) management, } \\
\text { intercultural communication, } \\
\text { and others } \\
\text { different initial conceptual } \\
\text { heritages: intelligence, or cul- } \\
\text { ture } \\
\text { various analytical foci: } \\
\rightarrow \text { components of CQ } \\
\rightarrow \text { locale of CQ: intrapersonal or } \\
\text { interactional } \\
\rightarrow \text { level of analysis: individual, } \\
\text { group, organization, etc. or } \\
\text { multi-level } \\
\text { different emphases on the end } \\
\text { pursuit of CQ and its related } \\
\text { context: adaptation or exploita- } \\
\text { tion of new, unfamiliar or di- } \\
\text { verse cultural context }\end{array}$ \\
\hline Methodology & $\begin{array}{l}\odot \text { utilizing methodological diver- } \\
\text { sity: multidisciplinary, multicul- } \\
\text { tural } \\
\text { ๑ exploratory social inquiry } \\
\odot \text { close ties with practices }\end{array}$ & $\begin{array}{l}\text { diverse paradigms of inquiry: } \\
\text { positivism, interpretivism, or } \\
\text { others } \\
\text { different theorization ap- } \\
\text { proaches: deductive or induc- } \\
\text { tive } \\
\text { different procedural emphases: } \\
\rightarrow \text { quantitative measurement or } \\
\text { qualitative evaluation of CQ } \\
\rightarrow \text { precision in explication or } \\
\text { depth of understanding }\end{array}$ \\
\hline Implication & $\begin{array}{l}\odot 3 \text { basic implications of CQ, vis-à- } \\
\text { vis research and practice } \\
\rightarrow \text { elucidation } \\
\rightarrow \text { assessment } \\
\rightarrow \text { cultivation }\end{array}$ & $\begin{array}{l}\text { different target and audience: } \\
\text { identification and selection of } \\
\text { the excellent or cultivation of } \\
\text { needed competence }\end{array}$ \\
\hline
\end{tabular}

Note: Author's integration from references cited throughout Section 6.5. 


\section{Conceptualization}

The most deeply rooted uniformity among cultural intelligence theorists is their mutual conviction of the concept's theoretical validity and practical value. As a novel construct in both the studies of intelligence and culture, the concept of cultural intelligence has been subject to the usual scepticism about and critique of its legitimacy and usefulness. Despite such negativity - and thanks in large part to meticulous seminal work (Earley and Ang 2003, Earley and Mosakowski 2004, Ng and Earley 2006, Thomas 2006, Thomas and Inkson 2003), the concept of cultural intelligence has received a relatively warm welcome in both academic and managerial communities since its prominence in two major academic venues (Academy of Management 2004, Group \& Organization Management 2006). Thus, the task for cultural intelligence inquiry now seems to be not validation but enhancement of its theoretical rigour; not illustration, but enrichment of its practical value.

Without exception, all cultural intelligence researchers and practitioners are also uniform in their elemental conceptualization of the three main constructs - culture, intelligence, and cultural intelligence. Indeed, one key motivation for all who advocate the significance of cultural intelligence is a conviction that culture is a complex phenomenon that matters in a fundamental way. In addition, a multi-faceted view of intelligence, following the tradition of the faceted perspective of intelligence (Davidson and Downing 2000, Gardner, Kornhaber, and Wake 1996, Kihlstrom and Cantor 2000, Marlowe 1986, Mayer, Slovey, and Caruso 2000, Sternberg 1997, Thorndike 1920, Wagner 2000) invariably informs all cultural intelligent theorists, who depart distinctly from the generalist view of intelligence and go beyond the cognitive aspect to take into account its other facets, particularly the behavioural and interactional ones. This leads naturally to their shared view that the basic characteristics of cultural intelligence are multidimensional and tacit.

Most interestingly, all major work on cultural intelligence to date has seemingly identified one identical construct as its key critical success factor: namely the ability to achieve robust open-mindedness. Ang and colleagues (2006: 118) identified the finding in their study that 'openness to experience was the only Big Five factor that was significantly related to all four aspects of CQ' as the most insightful. Thomas' elaboration on the importance and nature of 'mindfulness' expounded it as the key component in his conceptualization of CQ (Thomas 2006). This study's finding and the earlier theorizing on the cross-cultural heuristic also emphasise the importance of open-mindedness as the first crucial step in successful cross-cultural encounters. Admittedly, each perspective has coined different terminology for this construct - openness to experience, mindfulness, or negative capability - and has given varying treatments of it, yet the fundamental properties of this key capability are virtually converging and its preeminent place in the conceptualization of cultural intelligence is common to all perspectives.

The diversity in the conceptualization of cultural intelligence primarily reflects the differences in disciplinary background and conceptualization style, which inform each 
variant and influence its choices of conceptual emphases and analytical foci. The two underlying concepts, intelligence and culture, are inherently interdisciplinary by nature and when combined to foster a new construct, this characteristic is even more evident. The two pioneering groups, for example, have their origins in two different, albeit closely related, disciplines of industrial/organizational psychology (Earley and Ang 2003) and international management (Thomas and Inkson 2003), while within each group, interdisciplinary capabilities abound. Likewise, this study and its resulting XCQ perspective represents a fusion of the disciplines of organizational psychology and cross-cultural management.

The most obvious disciplinary influence is reflected in the difference in initial conceptual heritages of intelligence versus culture, which produced differently angled perspectives. For example, scholars like Early and Ang (2003), who have a background in industrial and organizational psychology, are well versed in, and offer valuable insights into, intelligence. Those with original interest in international and cross-cultural management, such as Thomas and Inkson (2003), by comparison, tend to initially concentrate on culture and its influence on management. Inevitably, though, the influences of the two original interests converge to complete the picture, which has also been the experience in this study, which started off with a combined interest in culture and learning and finally found the fusion of the two in the concept of XCQ. Thus, the interdisciplinary diversity among cultural intelligence theorists actually reinforces its theoretical rigour.

Numerous analytical foci then follow the diversity in conceptualization origins. Most notably, the detailed elucidation of cultural intelligence differs among variant perspectives. While Earley, Ang and colleagues theorized a four-factor model of cultural intelligence (meta-cognitive, cognitive, behavioural and motivational) (Ang, Van Dyne, and Koh 2006), Thomas and his team presented a similar yet distinct model of cultural intelligence, comprising three main components - knowledge (both of content and process), behaviour, and, the most crucial, mindfulness (Thomas, Elron, Stahl, Ekelund, Ravlin, Cerdin, Poelmans, Brislin, Pekerti, Aycan, Maznevski, Au, and Lazarova 2008). Although not oblivious of the interactional aspect of cultural intelligence, both of these two pioneering groups have so far focussed more on the intrapersonal side of cultural intelligence than on its interactional side. In contrast, this study, informed by a qualitative and interpretive approach, focussed solely on the interactional aspect of cultural intelligence and looked at cultural intelligence in its entirety, embedded in cross-cultural practices.

The different foci in the locale of cultural intelligence also influence the choice of level of analysis. For instance, because of their theoretical and methodological frameworks, Earley, Ang and colleagues and Thomas and his team have so far aimed to achieve precision by concentrating their efforts primarily on the individual level, although both perspectives have acknowledged that future work should expand the investigation to other levels such as groups and organizations. By contrast, the qualitative and interpretive model proposed here allows, or even compels, this study to si- 
multaneously take into account multiple levels of analysis. In fact, the very properties of intricate cultural webs provide a viable interpretive tool for deciphering competing meanings at both the intrapersonal and interactional levels, simultaneously even within the same phenomenon, allowing for multi-level analysis that is impossible under a precise quantitative lens.

Finally, different viewpoints on both the end pursuit of cultural intelligence and its related context are evolving. Whereas earlier views of cultural intelligence placed heavy emphasis on adaptation to new cultural environment (Earley and Ang 2003: 59), later work shows greater sophistication. For example, when Ang and colleagues reported their later efforts to enhance the precision of their four-factor model of cultural intelligence, their view of cultural intelligence has been refined to refer to 'an individual's ability to function and manage effectively in culturally diverse setting' (Ang, Van Dyne, Koh, Ng, Templer, Tay, and Chandrasekar 2007: 337, emphases added). Thomas and his team proposed an even more comprehensive view of the end pursuit of cultural intelligence and its context, viewing CQ as 'a system of interacting knowledge and skills [...] that allows people to adapt to, select, and shape the cultural aspects of their environment' (Thomas, Stahl, Ravlin, Poelmans, Pekerti, Maznevski, Lazarova, Elron, Ekelund, Cerdin, Brislin, Aycan, and Au 2008: 4, first emphasis original, the rest added). Apparently, the definition of XCQ as a facility or power engendering the manoeuvring of cross-cultural interaction to achieve desired and desirable results conforms to the above view of cultural intelligence that encompasses exploitation (which includes adaptation as a sub-set) of a context in which cultural diversity is a salient force. It is likely that as research and theorization intensify, diverse views of the exact nature and ultimate pursuit of cultural intelligence (adaptation versus exploitation), as well as the characteristics of its cultural context (new, unfamiliar, or diverse), will converge to form a complete and more in-depth view of cultural intelligence and its context.

\section{Methodology}

As suggested by the earlier discussion, the elements of methodological mutuality among the various cultural intelligence perspectives result from, or even rely on, a diversity of viewpoints and experiences. Research efforts in this theme encompass diverse disciplinary and cultural backgrounds, which is a much-sought after characteristic in cultural study (Adler, Doktor, and Redding 1986, Boyacigiller, Kleinberg, Phillips, and Sackmann 2004, Jackson and Aycan 2006, Usunier 1998). Nevertheless, despite impressive advances in recent years, work in cultural intelligence, given its young age and emerging sophistication, is still virtually in its infancy. As such, all cultural intelligence theorists must bear with similar scepticism and critique and unite in their conviction of its validity. Fundamentally still, all perspectives are methodologically uniform in the acceptance by all cultural intelligence researchers of the close tie between CQ and practice.

To date, the dominant methodological genre in cultural intelligence study has been primarily quantitative in nature, which is the key motivation for the contribution this 
study can make to the research stream. In brief, the research efforts of both pioneering groups were characterized by the post-positivist paradigm of inquiry, which informed their deductive theorization based on falsification theoretics, quantitative procedural approach, and meticulous quest for precision in research procedures and findings. By contrast, the perspective in this study examines the same concept within an interpretive paradigm that informs the inductive theorization and qualitative search for deep understanding. In this respect, this study stands to make a distinct contribution since its chosen methodological perspective has not yet been reported while offering a potential to explore theoretical elements unattainable via quantitative tools but achievable with qualitative procedures (Boyacigiller, Kleinberg, Phillips, and Sackmann 2004, Gephart 2004, Jackson and Aycan 2006, Wright 2004). Recent debate on research methodology has also increased attention to the potential of a mixed methodological genre as a way to increase the quality of the research process and finding (Tashakkori and Teddlie 2003). In the same vein, a complementarity achieved by combining a rigorous quantitative approach with a vigorous qualitative style will further enhance the rigour, value, and appeal of cultural intelligence as an emerging theoretical theme.

\section{Implication}

All work in cultural intelligence suggests it has implications along three basic dimensions with respect to both research and practice. First, there is a need for further elucidation of the nature of cultural intelligence and its key components and dynamics. Second, to increase its practical value, more effort should be made to develop reliable and robust tools and procedures for assessing the level and quality of a cultural unit's CQ (whether individual or group). Lastly, tools and procedures are also needed to aid the cultivation of $\mathrm{CQ}$ that can cater to a diverse set of practitioners. Obviously, the three implications are far from mutually exclusive, and most efforts in cultural intelligence study involve elements of all three, albeit with varying degrees and emphasis.

In fact, this variation in degree and emphasis actually constitute the diverse ways in which the various perspectives treat the three implications of cultural intelligence. Generally speaking, the pioneering researchers put greater emphasis on predicting and characterizing individuals based on their quantifiable cultural intelligence assessment procedures, primarily as an aid in identification or selection of individuals with a certain level of 'talent' in cross-cultural settings. The interest inspired by this study, by contrast, is broader in its target and audience. Specifically, the study suggests an adoption of an action-based learning perspective that seeks to understand the entire range of cultural intelligence as it is practiced by individuals in real-world settings. Accordingly, the whole range of individuals, from the culturally adept to the culturally inept, captures the attention of this study and its findings. Most particularly, this study advocates paying the greatest attention first and foremost on the majority group within this range - those ordinary individuals - in the hope that further findings can help this majority improve their cultural intelligence to a level that is at least adequate for their 
everyday lives. To return to the earlier analogy of driving, this study instils an ambition not to train a race-car driver but rather to help ordinary individuals learn how to drive efficiently and effectively when their daily life requires them to be on the road. With the current globalizing trend, the size of this majority group who must face everyday challenge of a cross-cultural encounter will only keep rising, reinforcing the demand for an understanding of their particular CQ cultivation need. Finally, this study puts forward the ultimate goal in cultural intelligence research: as we advance further, it is equally important to better understand the culturally inept and find an effective way to deal with such phenomenon, because this group arguably represents the prime source of cross-cultural crises and hence signifies the final frontier in cultural intelligence study.

NDEED, AS A NEWCOMER TO THE CULTURAL INTELLIGENCE RESEARCH SCENE, I HOPE THAT THE PROposed grounded theory of 'XCQ amid intricate cultural webs' has learned well from past effort and will add to future investigation. At the very least, the proposed theory contributes new and complementary insights to the ongoing research in CQ. More, the diverse perspectives on CQ represent variations of form rather than substance. Thus, the challenge in achieving complementarity is in essence a cross-cultural one, since it requires that the competing quantitative and qualitative research cultures interact effectively to exploit their uniformity and complementarity. Undoubtedly, much remains to be explored, most particularly this new view of XCQ - a prospect to be reflected on in the final chapter. For now, let the narrative of ZIA relates yet more realworld accounts of XCQ in action. 



\section{Chapter 7}

\section{Case ZIA}

\section{Unveiling a cultural chameleon}

In a sense, it is almost like there is no such word as 'no' in Thailand.

Adriaan Mannen

Founder, ZIA

2006

\section{. 3334 $\rightarrow 8 E$.}

We Thais are not too serious about working according to plan. I mean, we just work till it's completed. We know we'll have to get it done and that's it. But it's not that way with the Dutch. They don't just look at getting the project done, but it has to be done according to plan. Like if a project is assigned 80 manhours, then you have to get it done within that assigned hours. So, in the beginning, you can get a bit frustrated and stressed out.

Somphob Raksakul Senior Project Managers, ZIA

2006

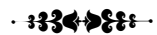

In the beginning this frustrated you, because the things you wanted done wouldn't happen. And then you talked to the people only to find out they didn't understand you. And then you asked them, why didn't you ask me, because I would be more than happy to tell you twice or to tell you ten times if you didn't understand. And then they still wouldn't say anything - they just smiled!

Fons van Beek Regional Director, Asia-Pacifc, ZIA 


\section{Reflection 17: How the chameleon fooled and charmed me}

ZIA turned out to be the hardest case to crack. This case haunted me days and nights for months on end. I could say this was the case that was responsible for moving my thesis delivery deadline forward and forward and forward. It was not good for my sanity at all. I would be fooled to think I got it made - and kept on transcribing and re-reading the transcripts, naming the case and renaming it, drawing the outline for the analysis and rearranging it over and over, starting to write and re-write only to erase what I'd written until I could go crazy with it.

The only good thing that came out of this torment was that 'finally' I really got it. So, without even intending to, I was actually letting the strict emergence principle of Glaser dictate my analysis. Somehow, I had to let the evidence shape the theme of the case, and not the other way round - which would have made my life a lot easier, though not necessarily better, as I now realize.

And when I 'got it' I felt a strange sense of gratification I imagine a crime detective must feel when he or she finally pins down a culprit. I ordered home-delivery pizza to go with a nice glass of wine that day to celebrate the 'light at the end of the tunnel' because I knew intuitively then that this study was getting close to its completion.

Now when I look back, how I cracked this case was indeed a good reflection of how a cultural chameleon can fool and charm you. I wrote my reflection on the character of Khun Adriaan as being chameleon-like long before I realized that my impression of him was indeed what the case was all about. For a long period, I tried to analyze Z|A as a cultural hybrid, based on a collection of outward signs - the apparent congenial atmosphere, the actors' affinity towards each other, and so on. But when I looked closer, the real evidence didn't really fit that scheme, however hard I tried and tried to shape it that way. In the end, I gave up. I stopped looking at the case for a long time, turning to work on some other parts of the manuscript.

I was thus kept sinfully happy for a long period while I tried to forget about this stubborn case. Until I knew it's high time I got the manuscript completed. I returned to the case I had forgotten. With a more or less blank space in my conception of it, I took a good look at the evidence again and all of a sudden, I spotted the chameleon that had been lurking there all this time.

I hope I did a decent job of unveiling this intriguing creature to my readers in the following tale of a cultural chameleon. 
Diagram 7.1: ZIA fact sheet (As of 2008)

\begin{tabular}{|c|c|}
\hline Industry & industrial automation \\
\hline Product/service portfolio & $\begin{array}{l}\text { total process control and management of industrial } \\
\text { automation solutions (hardware and software) }\end{array}$ \\
\hline Market portfolio & serving industrial firms from numerous industries \\
\hline Core competency & $\begin{array}{l}\text { project management to deliver integrated solutions } \\
\text { for process control and industrial automation }\end{array}$ \\
\hline Operating portfolio & $\begin{array}{l}\text { turnkey projects for process control and industrial } \\
\text { automation, project management and consultancy } \\
\text { services, hardware engineering services, software } \\
\text { engineering services, control panel assembly services, } \\
\text { commissioning and start-up, maintenance \& support } \\
\text { services, and training }\end{array}$ \\
\hline Relative size $^{1)}$ & $\begin{array}{l}\text { small, although recent acquisition made ZIA part of a } \\
\text { large, privately own, Asian conglomorate (ZIG Group). }\end{array}$ \\
\hline Headquarters & $\begin{array}{l}\text { group headquarters (ZIG Group): Hongkong } \\
\text { corporate headquarters (ZIA): Bangkok, Thailand }\end{array}$ \\
\hline Divisional offices & $\begin{array}{l}\text { globa (ZIG Group): a portfolio of corporations in many } \\
\text { industries with 60+ offices in } 15 \text { countries across Asia; } \\
\text { for ZIA: a network of offices or professional teams in } \\
\text { the Philippines, Vietnam, Malaysia, Indonesia, China, } \\
\text { the Netherlands, Belgium, Germany, UK and Slovakia; } \\
\text { and a strategic alliance with a US system integrator }\end{array}$ \\
\hline Corporate culture & $\begin{array}{l}\text { largely centralized vis-à-vis strategic decisions but } \\
\text { fairly distributed vis-à-vis tactical activities }\end{array}$ \\
\hline Workforce $^{2)}$ & $\begin{array}{l}\text { global (ZIG Group): } 10,000+ \\
\text { for ZIA 200+ (In Thailand: } 60+\text { ) } \\
\text { [1 expatriate Regional Director Asia; 3-4 expatriate } \\
\text { engineers, and professionals,; } 10 \text { Thai engineering or } \\
\text { professional staffs; } 40+\text { Thai supporting staffs] }\end{array}$ \\
\hline Manner of entry into Thailand & semi-accidental, following a commissioned project \\
\hline Operating portfolio in Thailand & total project management work \\
\hline $\begin{array}{l}\text { Years in business } \\
\text { Years in Thaland }\end{array}$ & $\begin{array}{l}100+(\text { ZIG Group) } \\
12 \text { (ZIA) }\end{array}$ \\
\hline
\end{tabular}

Note: 1) defined very loosely as small, medium, and large, based on rough benchmark vis-à-vis overall global operation of the entire corporation relative to average multinationals.

2) as of 2007 
ONS VAN BEek, DUTCH TOP EXECUTIVE OF ZIA, A BUDDING INDUSTRIAL SYSTEM INTEGRATOR IN Thailand, could not help smiling when he thought of the prestigious award his company has just received from the Netherlands-Thai Chamber of Commerce (NTCC). To be awarded the annual NTCC Business Excellence (SME category) indeed made his already gratifying life in Thailand even more delightful. He had come a long way, since he and his boss-cum-business partner, Adriaan Mannen, ventured their dream and set up the forerunner of ZIA in this charming land of deceptive smiles (one that were often hard to decipher and sometimes gave him such a headache).

Adriaan was here no more and their small venture had now a new name, a new building, a new owner, and a new identity. You could hardly recognize it from the humble establishment he and Adriaan had dreamed up. Yet, Khun Fons could not help feeling that his business partner's spirit was still in the air of the place he walked in each day to keep it thriving. The more things changed, the more they remained the same. Just like Adriaan, Khun Fons pondered, ZIA had become a chameleon - ZIA fit as best it could with whatever environment it ventured into, yet deep inside its core spirit remained intact.

He could not say right away whether this was for better or for worse.

It all depended really.

For what was in front of his eyes now, it had been not bad. Business was thriving, ZIA was gaining ground, he was Dutchfully satisfied with his work, his people and his life in this peculiar place.

Despite the Thai's countless quirks, or maybe because of them, Khun Fons would not say he had a bad life since he arrived in Thailand a little more than a decade ago. His lovely Thai wife had just given birth to their first child and the 'baby' of a company he had formed with Adriaan was now a prolific young force in the Thai business scene, with lots of prospect globally ahead. Khun Fons thought appreciatively of Khun Adriaan, the chameleon who had moved on.

How would this chameleon ZIA move forward, he wondered. Now - that would be interesting to watch.

\subsection{Dutch seed stumbling on Thai soil}

ZIA is a company whose Dutch seed was planted, nurtured, raised and transformed on Thai ground, by successive biological as well as foster and adopted parents of myriad national backgrounds.

ZIA was a total solution industrial system integrator, providing complete, mostly turn-key, solutions for industrial system automation - from set-up to operation and maintenance. ZIA services included design and integration of computer hardware and software for industrial automation, as well as consultancy, project execution and management for industrial firms in diverse industries. ZIA was a relative young and small company, although via recent acquisition by a long-established Asian-based (of European heritage), privately-owned group - the ZIG Group, it had became by the time of 
this narrative a new addition, albeit a relatively independent one, to a very large Asian multinational.

The biological root of ZIA stemmed virtually from two Dutch entrepreneurs who came to Thailand as employees of a Dutch industrial automotive consultancy to carry out a major project commissioned to their firm by a well-known Anglo-Dutch multinational in consumer products - Unilever. Initially, the two engineers came to Thailand simply to execute a new industrial system for Unilever's Thai plant in the early 1990's. From that project, they gained knowledge of the local environment and saw potential business opportunities. Having during these early years built up a good network, presence and reputation among a circle of other well-known Dutch and Western companies, they decided to set up a new venture for themselves to explore the attractive prospect.

And so the new company, MPE Europe - whose first initial was in honour of the key founder, Dhr. Mannen, was established in mid 1990's, followed by its subsidiary, MPE Thailand in Bangkok, to exploit business opportunities in the Asian region. Although MPE Europe also developed into a thriving business in the Netherlands and Europe, it was the business experience and opportunity in Thailand that virtually instilled in the Dutch founders an entrepreneurial spirit to start a new venture by themselves in a continent hitherto foreign to them.

\section{A new adopted Asian parent}

Around 2005, after less than a decade of successful operation, MPE Europe decided to sell fifty percent of its share in MPE Thailand to a larger company - ZIG. ZIG was an affiliated unit of a very large but privately-owned Asian multinational, the ZIG Group. Interestingly, the history of the ZIG Group resonated with that of MPE. The group started off as a small business venture established in the Philippines by a Swiss entrepreneur a century ago. Since then, it had grown into a huge, though still privately owned, multinational, with strong presence and operation throughout Asia and in diverse sectors. ZIG was an independent unit within the larger ZIG Group and operated in industry sectors that were closely related to the core business of MPE - hence the alliance.

Not long after the initial alliance, MPE Europe decided to sell off the remaining share to ZIG but with special arrangement that the management and operation of MPE Thailand would still remain closely tied to the Dutch MPE. The main founder of MPE, Dhr. Adriaan Mannen, headed the operation of MPE Europe but still maintained an active supervisory role for MPE Thailand, while his colleague, Dhr. Fons van Beek, took the helm of MPE Thailand. For a short period, this arrangement was maintained, with the company operating under the old name of MPE. Eventually in 2006, the tie with its biological Dutch parent was effectively dissolved and MPE Thailand changed its name officially to ZIA to signify a new bond with its adopted parent. Soon after that, ZIA started to establish its own presence in the Netherlands and Europe under this new 
identity and MPE Europe continued to operate its business in Europe independently of ZIG. The Dutch seed had thus emerged as a full-blown tree with a life of its own.

\section{An evolving identity}

In layman terms, ZIA business was simply to set up an industrial plant and help run it smoothly. ZIA had extensive experiences in process control system and industrial automation in the following industries: food \& beverage, logistics \& packaging, consumer products, chemical industry, automotive industry, pharmaceuticals, and heavy industry. As with all consultancy and project management businesses, ZIA operated in an environment where trust built on past reputation and relationship was the key quality. As such, a strong identity was crucial to its success. The company had accumulated a good network of clients from its past projects and with the new name and support from an established adopted parent, ZIA intended to expand its business further. ZIA clients were primarily large multinationals with global operation, which offered an automatic prospect for expansion to ZIA.

As of 2008, ZIA office in Thailand served as the regional office for its operation in the Asian region. This came about for historical as well as business reasons. First, MPE Thailand started its operation in the Asian region from Thailand. At the same time, Thailand was also considered a good focal point for branching out, in terms of logistics and infrastructure. Equally important, Thailand had a good number of potential international clients for the company to build and expand its business.

The Regional Director, Asia-Pacific, Dhr. Fons van Beek, was stationed at the headquarters in Bangkok and was now in charge of ZIA operation for the entire Asian region, which had now over 200 personnel. ZIA Thai office had a total of 5 expatriates and about 60 local Thai employees. Beside a few administrative personnel, the majority of ZIA Bangkok workforces were engineering or technical professionals. Basically all of the expatriates were at higher levels or functions. This ratio of foreign and Thai staff was designed to reflect a balanced mixture of advanced (Western standard) technical and project management expertise with local character. Specifically, since ZIA's client base made up mostly of Western, mainly European, a strong presence of Western and European personnel worked more effectively to establish good impression and rapport with its clients.

As one of the two founders of the former MPE Thailand, Dhr. Fons van Beek had been involved in the Asian side of the company ever since its conception. Because MPE Thailand had now assumed a new identity of ZIA, Dhr. van Beek was thus in charge of making this identity a prosperous one. Winning the annual NTCC Business Excellence (SME category) only after a decade since he and his boss-cum-business partner started this venture thus helped boost up his spirit a great deal. 


\section{The Actors}

\section{Founder: Dhr. Adriaan Mannen (Khun Adriaan)}

Dhr. Adriaan Mannen, a Dutch entrepreneur in his 50's, was the key reason why ZIA came into existence. As a project manager at a Dutch company he worked for in the early 1990's, he took charge of a large industrial automation for a major client, Unilever, to set up a new plant in Thailand. Prior to that, Dhr. Mannen had had extensive experiences managing industrial projects in various overseas environments. It was in Thailand, however, that his entrepreneurial drive came into effect. He sold the idea of setting up a new company to another engineer (Dhr. van Beek) who went to Thailand as part of his team, and together they set up MPE in Europe and Thailand. Dhr. Mannen was the Managing Director of MPE Europe but worked extensively with MPE Thailand, until the final turn of identity. Since then he continued exclusively with MPE Europe. His legacy in ZIA, though, could still be easily discerned at present day ZIA.

Dhr. Mannen is referred to as Khun Adriaan in the narrative.

Reflection 18: Khun Adriaan - a cultural chameleon

For some reasons, Khun Adriaan did not make an impression on me as a Dutch at all. It was quite strange that his mannerism and personality reminded me more of a chameleon individual and I could not quite pin-point his (national) cultural identity other than that he was 'probably' European. His other cultural traits - I could not even begin to decipher. He was unusually casual and diplomatic, which was not that common in a Dutch. He displayed quite a subtle level of understanding of the Thai culture, far beyond the normal guidebook characterization. He made me feel at ease, and yet I had a feeling that he actually understood other people more than other people would understand him, unless he wanted them to. His one Dutch trait that I saw clearly, though, was his obvious practical outlook. His deftness at combining his understanding of other cultures with his practical pursuit probably served him well in his venture in Thailand and beyond.

Compared to the first two cases of ADT and CDW, it took me far longer to analyze the case of ZIA. This has been the most difficult case to decipher and when everything 'finally' fell into places, it suddenly dawned on me that the case was as hard to crack as a chameleon is hard to spot. ZIA, like Khun Adriaan, was a real challenge to decode.

\section{Regional Director - Asia-Pacific: Dhr. Fons van Beek (Khun Fons)}

Dhr. Fons van Beek was one of the two founders of MPE and continued with the enterprise all through its metamorphoses. With a keen interest in exploring different adventures and cultures, he readily joined Khun Adriaan in setting up the new venture in Thailand. Since then, he had been at the helm of the enterprise until present day. In the beginning, he managed the Thai office by alternating his presence between the Netherlands and Thailand, even after marriage to his Thai wife, whom he met during the first few years in Thailand. Eventually, he and his wife found that it was too tiring 
to alternate living in two countries all the time and, interestingly, they decided to settle down in Thailand, at least as long as he would be managing ZIA.

An engineer by training, Dhr. van Beek's functioned now as a typical executive, overseeing all aspects of the operation and involving more with ZIA's strategic and management issues than with its daily operation. A self-confessed firm believer in cultural sensitivity as the key to success in cross-cultural life (professional as well as personal), Dhr. van Beek had a lot to say about cultural differences and how these can be challenging as well as rewarding. He admired the 'multicultural' trait that he saw in his former boss and colleague, Khun Adriaan, and tried to continue that legacy at ZIA.

Dhr. van Beek will be referred to as Khun Fons in the narrative.

\section{Reflection 19: Khun Fons - karaoke and all ...}

Khun Fons was surprisingly friendly and casual, for a Dutch and a Dutch executive. He struck me as someone who genuinely appreciated the Thai culture for what it is and not for the idealized portrayal of it. He seemed to like living with the Thai in spite and because of all its idiosyncrasies that easily annoy many of his Dutch countrymen. What I really liked about his attitude was that he did not treat the other culture in a patronizing manner but in a characteristic egalitarian Dutch spirit. The way he described how awkward his first 'karaoke' session was and how that amused and perturbed him at the same time was an interesting observation in my eyes, particularly because I personally abhor the 'karaoke' craze. In many instances, he seemed to 'appreciate' the Thai for what they are more than I do and that remains a nice riddle with many possible interpretations with regards to my own cultural identity.

\section{Operation Director: Dhr. Ralf Kellerman (Khun Ralf)}

Dhr. Ralf Kellerman was a Dutch in his forties. He was the 'third' Dutch expatriate for ZIA, having worked with Khun Fons from the very beginning when ZIA only operated as a satellite unit for the Dutch headquarters. In the first few years, Dhr. Kellerman managed the Thai operation in alternation with Khun Fons. They would take turn to come and stay in Thailand for two to three weeks at a time. Once the business grew too big for such an arrangement and ZIA Thailand was firmly established, Dhr. Kellerman, like Khun Fons, eventually decided to settle down in Thailand. He was also married to a Thai and as long as the business prospect of ZIA remained attractive, Dhr. Kellerman would not make any specific plan regarding his future settlement.

Dhr. Kellerman was an engineer by training and he stayed in charge of the entire engineering operation of ZIA. He acted basically as the right-hand man for Khun Fons. His main concern was thus to keep the engineering operation function as efficiently and effectively as it could be to foster business success.

Dhr. Kellerman will be referred to as Khun Ralf in the narrative. 


\section{Reflection 20: Khun Ralf - a true engineer}

I remember my meeting with Khun Ralf to be relatively short and did not expect much when I started transcribing and analyzing his narrative. I was surprised to find out that, a man of few words though he was, his words were succinctly 'engineered' and he provided more evidence for my study than I first expected. He was an engineer through and through - in his manner, his thinking, his professional approach and his attitude towards life. Although he was married to a Thai, I don't think that he is married to the culture, and that in some way pleased me. As a Thai partner of a Dutch, I am wary of the fact that some 'farang' husbands, and their Thai partners, seemed to be attracted to their partners' culture more than to the partners as individuals. Khun Ralf's response to my question about how he saw his future revealed that he was married to a lady who happened to be a Thai, rather than the other way round. Somehow that struck the right chord with me.

\section{Sales Manager, Industrial Project: Dhr. Danny Taridise (Khun Danny)}

Dhr. Danny Taridise was a Belgium in his early thirties and a man with a clear mission. After several years of educational and professional training at Dutch universities and firms, Dhr. Taridise had evolved into a classic Dutch trader, despite his Belgian origin. Trained as an engineer as well as a manager, his early career had been to work for a few Dutch firms in the Netherlands, one of which was MPE. A self-described adventurous spirit, Dhr. Taridise contemplated a wish to 'move around the world' and proceeded with it in a quite methodologically Dutch manner. First he pondered his early touring experience in Asia in conjunction with business, cultural and social prospect of a few possible candidate Asian countries where he could move to. Then, he decided that, all things considered, Thailand came out as the best candidate. Yet, before he finally took the plunge, he spent a 'road-test' period actually living in a remote area in Thailand in order to make sure he could survive there long enough to thrive in his chosen new venture.

He finally moved to Thailand in the early 2000's and started searching for a good position. He decided to take the offer from ZIA, even though it was actually the least attractive financially. But, as he put it, he 'had very good feeling with Fons' and found ZIA organizational environment to be compatible with his spirit. Dhr. Taridise was in charge of sales for ZIA industrial projects, which demanded a great deal of delicate negotiation with clients in combination with efficient management of his sales team. Although he would not commit to a promise to stay forever with ZIA, he was very satisfied with his work for the company and happy to see the prospect ZIA was developing for itself and its people. He did not anticipate leaving this young adventure any time soon.

Dhr. Taridise will be referred to as Khun Danny in the narrative. 
I quite enjoyed the long interview I had with Khun Danny. Like most salesmen, Khun Danny could certainly keep a lively conversion. He was very coherent and methodological in his articulation, with ready answers for whatever questions you may put forward. His business and analytical style appeared very Dutch to me; yet his mannerism was not at all Dutch, and when he said he was in fact a Flemish Belgian, I was not surprised. Still, he did not quite resonate with the many 'typical' Belgian I had met earlier either. He was the unDutchable Belgian - 'my Dutch son' to his Belgian father and a curious cultural hybrid in my collection of evidence.

His straight characterization of ZIA as 'a Thai company with foreign management' lurked at the back of my mind for a long time before I started to see its implication. Interestingly, it was not in the many conscious analyses he gave about the Thai culture, but more in the various unconscious 'reminiscences' he reflected that I found interesting evidence to corroborate many concepts I was pondering on in my analysis.

\section{Senior Project Manager: Khun Somphob Raksakul (Khun Somphob)}

Khun Somphob Raksakul was a Thai in his late thirties. He was a technician by formal training, with a diploma in electronic engineering and technology from a well-known Thai community college. Before joining ZIA, he had had extensive experience working on industrial automation projects with a local Thai firm for a full decade. He joined ZIA as one of the first few local employees and had been with the company from its very beginning, through thick and thin, so to say.

He started with ZIA as an engineer, working closely with Khun Fons and Khun Ralf for all the projects ZIA took on in its early days. From a company of only 5 or 6 people in total, ZIA had grown fast over the years and Khun Somphob had also grown with it. He was promoted to be project manager, in charge of all aspects of project management, from procurement to delivery. As the business grew further, project management work was reorganized and divided into two major sections: software and hardware. Khun Somphob was at the time of this narrative in charge of the hardware side of the engineering operation. He worked closely with another senior project manager, a Dutch expatriate, who took care of the software section. Khun Somphob still reported directly to Khun Ralf and Khun Fons, whom he had developed a comfortable working relationship with over the years. With the network of clients he had built up during the years working for ZIA, he also helped with sales and marketing, particularly in connection with clients who already knew and trusted him.

Khun Somphob was thus a key Thai personnel and his function extended beyond the official job description of a senior project manager. To both the Dutch executives and his team, Khun Somphob was a valuable 'link'. Khun Somphob worked under the principle that loyalty to organization always came first and he believed this was why he was deemed valuable to ZIA. 


\section{Reflection 22: Khun Somphob - mediator or adaptor, or both and more?}

Khun Somphob struck me as a decent, humble person who knew his worth but not to such an extent that it would make him full of himself. Given the significant role he played at ZIA and his interpersonal capability. I was curious to see that he was not so conscious about this fact. It was more from my talk with others at ZIA that I realized how Khun Somphob was highly respected and regarded by his bosses, colleagues and subordinates alike. To his people, he was their de facto 'mouthpiece' as Khun Nicki put it. They went to him when they needed to vent or plead to the Dutch side because they trusted that Khun Somphob would dare speak up for them. To his Dutch bosses, he was their 'middleman' who provided the needed link that they could trust.

I believe Khun Somphob won over both sides because of two qualities: humility and sincerity. When he talked about the Thai people who worked under him, he always referred to them as 'nong nong' - a Thai term that literally means 'my brothers and sisters'. I felt he sincerely cared for them as if they were a family and I had no doubt that his 'nong nong' must have felt that way too. He also talked of the Dutch bosses with respect and sincerity. He viewed them with realistic appreciation, willing to acknowledge both their superior and inferior elements.

I think that a nice study could be done on characters like Khun Somphob - ones who operate as successful human cross-cultural instruments. It would be interesting to see what qualities make such a character, because I do believe they are among the top when it comes to high $X C Q$.

\section{Marketing Executive: Khun Nattha Kanchanasakul (Khun Nicki)}

Khun Nattha Kanchanasakul was a newly graduated marketing executive in her early twenties. Referred to amicably by her boss and colleagues simply as 'Nicki' and modern enough herself to refer to her top boss as just 'Fons', Khun Nicki fit well with the Dutch management attitude of ZIA. She had a bachelor degree in Education from the top university in Thailand, Chulalongkorn University, which put her right at home with the crème de la crème of the educated Thai ${ }^{29}$. She then obtained her master degree in

\footnotetext{
${ }^{29}$ As a side note, it should be pointed out that to be admitted in either of the top two universities in Thailand - Chulalongkorn University (where Khun Nicki attended) and Thammasat University (my alma mater) is considered a great accomplishment in the Thai society.

In Thailand, admissions to public universities (which are considered more prestigious) are done through an annual nation-wide examination, and seats are assigned based on students' performance in that one event. Students are designated to schools and departments based on a ranking system; thus top students go to top departments in top universities, and so on so forth. Students often devoted their years in high school to this sole purpose and a case of suicide by one who could not get in the place of his or her dream is not unheard of, reflecting a strong social pressure associated with this curious phenomenon. By contrast, Dutch educational system is typically well-organized, with procedural mandates that make sure each student's educational competence and potential is systematically assessed and planned ahead from very early years.

This presents yet another interesting comparative analysis of the two cultures.
} 
information technology management from a British university, right after her graduation from Chulalongkorn University.

Khun Nicki appeared very fluent in English and got her first job partly because of this quality that is so highly prized in Thailand. Khun Nicki accepted a job in marketing with a well-established large foreign corporation even though she initially wanted to work in IT management. She then realized that she actually had a talent in marketing work which she found to be very interesting. After two years with a big organization, Khun Nicki sought a more exciting challenge and joined ZIA to work directly under Khun Fons, a situation she found to offer a good learning opportunity for a young force like herself. She was put in charge of the entire marketing programme for ZIA, where she worked hard and enjoyed the challenge assigned to her. She had all the drive of her generation and, at the time of this narrative, was anxiously awaiting an interview for admission to an evening MBA study programme, again at her former university. She hoped to be one of the top ten percent contenders to be finally admitted to the programme. If that happened she planned to major in marketing. In any case, she was set to be on the way up the career ladder.

\section{Reflection 23: Khun Nicki - the face of things to come?}

To me Khun Nicki represented a new crop of Thai workforce - young and fresh, welleducated, highly motivated, and fully familiar with the Western ways of things. Even her nickname reflected this new sharp attitude that seemed to characterize many Westerntrained Thais whose nicknames sounded more Western, either because of the modern temperament of their parents, or the creative invention of their own imagination. Her profile was one that was emerging more rapidly in Thailand. She represented the next generation.

She was certainly the only Thai I interviewed who referred to her top boss without any preamble whatsoever, not even a 'Khun'. I was impressed by how at ease she was talking about Khun Fons, in Thai to me, as simply 'Fons'. I don't believe she was even conscious of the fact, as it seemed to come very naturally to her. This reflection made me wonder about myself. I also call my $\mathrm{PhD}$ promotor and supervisor simply by his first name, but only when I am speaking in English or Dutch. If I were to talk about him in Thai, to a fellow Thai, I would most likely refer to him as 'Acharn Friso' - which is a proper Thai way to respectfully address your professor ('Acharn' is a cordial word for teacher, mentor, or professor).

What does this say about my own cultural identity? I am still wondering about it even now.

\section{Supporting cast}

ZIA had only a few more expatriates, most of whom were Dutch engineers taking charge of various projects, including the project manager in charge of the software team who was Khun Somphob's counterpart and the only other Dutch expatriate sta- 
tioned permanently in Thailand. A few Thai senior project managers headed smaller teams of junior Thai engineering and technical staff working on various projects. The core group of Dutch and Thai staff that worked together on a regular basis, however, evolved around the major six actors portrayed above and the one Dutch project manager $^{30}$. Communication between the Thai and the Dutch offices happened mainly among the Dutch expatriates and their colleagues in the Netherlands, although some senior Thai staff occasionally visited the Dutch site as part of training or project work.

\subsection{A cultural chameleon in disguise?}

ZIA was as entrepreneurial and multicultural as a small company can get. It was brought to life in Thailand by Dutch entrepreneurs, adopted by an Asian multinational whose origin traced back to a Swiss entrepreneur. Yet, the most poignant characterization of ZIA had to be its independent spirit. Externally, ZIA operated and tendered its expertise with full autonomy from suppliers, hence promising the best integrated solutions to clients without the need to 'push' any particular product line. Internally, ZIA had a culture that was distinctively independent in diverse aspects. At first glance, ZIA appeared to exhibit outward signs of a cultural hybrid. Yet, with further probing, ZIA turned out to be more of a cultural chameleon. Its misleading hybrid facade was only a fabrication of its chameleon ability.

This here is a mysterious case of many cultural chameleons in disguise.

\section{The indelible pervasiveness of Adriann}

Khun Fons sat in his new office and looked around the place with contentment. ZIA had only recently moved into this new office complex to accommodate its growth. The old office had become too small for future prospect. Of course, ZIA remained a relatively small company, but it was fantastically dynamic, growing rapidly and fast gaining ground in an industry that was a hallmark of technological advance, in a region where things were happening and expanding. Soon after they moved into this new place, ZIA also took a new turn, joining the ZIG Group and acquiring a new identity. All this in just about one decade of sheer hope, hard work and resolute determination. Sadly, this recent change had come with having Adriaan - his friend, business partner, and mentor - virtually and finally cutting his tie from the one venture they created together. Khun Fons had no doubt that Adriaan was bustling with energy at this very moment in his other ventures elsewhere.

Still, even today, Khun Fons swore he could still feel the essence of Adriaan in the ZIA air. The company had the imprint of Adriaan everywhere you would look: in its familial spirit, its accommodating manoeuvring, and its core business principles.

\footnotetext{
${ }^{30} \mathrm{He}$ was on a long business trip outside of Thailand during my field visit and thus not available for an interview.
} 


\title{
We are family.
}

First off, Khun Fons would say without any hesitation that the Adriaan he knew was 'somebody with a high level of social intelligence'. He had that ability to make people felt that he really cared, as if they were his family - that it was not just the business they had together, but something more personal and lasting. Born and raised in the famously more congenial and sociable part of the Netherlands, the Brabant district, Khun Fons easily appreciated and admired this trait in Khun Adriaan.

Thus, Khun Adriaan and Khun Fons put effort into making ZIA feel like a family to everybody working there. They both saw it as the best way to manage a business in the Thai culture, where people work to live and not live to work. They learned from their earlier Thai employees like Khun Somphob that this familial spirit was the key to win the heart of Thai staff. And in Thailand, you needed to win their hearts for them to want to work the way you wanted them to. In the earlier days, when there were only two Dutch and three or four Thai staffs working together, it was easy to get to work like a family. The small group, the project work and the constant travelling together created the feeling of 'gezelligheid' ${ }^{31}$ - a natural bond. In many of the dinners and lunches Khun Fons and Khun Ralf shared with Khun Somphob, they had a chance to listen to Khun Somphob who kept telling them,

The Thai like to look at work not just as work. We like to work like a pi-nong [brothers and sisters], like a family. This you have to understand. We think this is important. We have to think of each other as more than just co-workers.

So family it was. It had been this way from the start and it was still this way now, even though ZIA was no longer a cosy group of seven people. Khun Fons still did his best to make it clear to all in the family, from senior expatriates to lower-ranked Thais, that they were one family and had to 'take care of each other'.

Khun Danny, for example, decided against better offers from other companies based simply on his gut feeling about ZIA.

\begin{abstract}
I had very good feeling with Fons. He came from near where I lived. To be honest, it was the worst proposal that I had from all the four companies I talked to. But I thought if I were to move to live in Thailand alone, the environment of the company was very important, because I have nobody here. And after two and a half years later, I don't regret that for one moment. And the other companies are now my customers, so that turned out real good.
\end{abstract}

Although the realist in him would not allow Khun Danny to declare he would stick forever with ZIA, he must admit he had a very warm feeling for this place. He felt like he was 'growing with the company' and it was a very good feeling to have coming to

\footnotetext{
${ }^{31}$ Two Dutch words - 'gezellig' and 'lekker' - are those words that virtually defy accurate translation. Gezellig is similar to the English cosy, while lekker is equivalent to delicious. These are the two Dutch words that I personally find to be the most subtly indicative of the Dutch culture (just like the Thai 'kreng jai' and 'mai pen rai'). One other favourite Dutch word of mine is 'leren', which is used for both to teach and to learn - a very tellingly egalitarian and perceptive view of education and learning. It always endears me to hear my Dutch colleagues and friends using the English word 'learn' when they mean 'teach'. Talk about how deeply culture influences you!
} 
work, especially when it was not always real 'gezellig' to deal with the quirkiness of his Thai colleagues or subordinates.

Khun Danny admitted he made mistakes, many times, trying to find his way in this foreign land full of strange people whose behaviour never failed to amaze him. He still could not get over the fact that many of his Thai colleagues seemed to care so much about the ranks next to their names in the business cards but never gave a thought to having a tidy office. While Khun Danny always planned his life methodologically, working hard and steadily so he would one day have a nice house, a good home and a comfortable life, most of his Thai colleagues would care more about how they could afford to buy a fancy car, only to drive back daily in horrific traffic to a sorry place they thought of as a home. And to think that the cost of a not-that-spectacular sedan in Thailand would have easily got them a proper, real house! How bizarre!

Well, there were many other things Khun Danny still could not comprehend about the Thai. But when it came to working with them, here in this 'family', he knew he had to do his best to at least convince them that he meant well. And he did, of course, because to him, it was the business that was the family. So he put up with whatever irritating feeling he might get from the Thai and concentrated instead on getting his sales team to work the ZIA way.

Khun Nicki also joined ZIA because of the caring spirit and good career prospect it offered. She felt that she was given room to breathe and create. For a young, virtually fresh graduate with no experience to boast but lots of drive and initiative, she could hardly get a better chance than at ZIA to prove herself. She couldn't imagine being given the responsibility to look after the entire marketing scheme for the whole company and reporting directly to the very top boss in any other company she knew, certainly while a young, fresh graduate she was. At least not where she worked before, and definitely not where many of her friends worked now. But here, they treated her like a 'nong' - a sister they wanted to help grow. That was the best part of it, she felt. People did not patronize her, but they made her feel she had a chance to grow here.

She respected her Dutch bosses and colleagues, Khun Fons and all other expatriates, not because they were 'farangs' but because she genuinely believed they were good at what they were doing, yet still willing to help her and her Thai friends learn and grow. It helped of course that her English was above the Thai average so she could communicate comfortably with the Dutch bosses and colleagues. It certainly also didn't hurt that she was not a typical timid Thai lady who only wanted to keep quiet. So, here at ZIA she could be her daring self - dressed up to work in a hip style instead of the boring Thai office costume, speaking her mind to the bosses and peers, and experimenting with new marketing schemes to exercise her talent. She felt at home here.

\section{Karaoke - anyone?}

Home was not built that easily though, if you asked Khun Fons. It took a lot of effort to muster up and carry on this level of comfort and trust. It took a great deal of awkward 
tweaking and delicate humorous twist. Thinking of this always reminded him of the moment he first fell prey to the karaoke craze of the Thai. It caught him by surprise when he was invited to take the grand honour of taking up the microphone to open the karaoke 'podium' in one of the first social events ZIA organized. Somehow he knew the invitation was extended to him as a gesture of respect for being the very top boss. Somehow he knew he had to take the microphone and started singing to the tune. Somehow he managed to do just that, and felt 'Dutchfully' ridiculous in the process.

But then, he took a look around and realized nobody there cared how his singing was, at least not any of his Thai employees who only sang along and looked more absorbed in the festivity than in his talent at singing. They were laughing both at and with him all the time he was singing. He couldn't imagine himself doing this in the Netherlands, not in this life time, for sure. He would be the talk of the town. Yet, here it was no big deal. They actually enjoyed the ridiculousness of it all.

So Khun Fons learned the lesson of a good karaoke session. It was how the Thais enjoyed themselves. Perhaps it was the same as when the Dutch paid to sit and watch a good cabaret by a great performer. The Thai, for whatever absurd reason, apparently liked also to perform and not just watch - never mind how some of them were not really stage material. All the more fun it seemed, when you had both someone who could sing like a pro and then many others who only made a fool of themselves trying to. Khun Fons now had almost no qualm taking up the microphone, even though he would still felt a bit embarrassed inside. All the same, he could just wing it and even felt proud to be always given the honour to open a podium.

Indeed, he realized Adriaan and he had actually turned this karaoke craze to their advantage. Adriaan had established a social tradition at ZIA, to have regular social events sponsored by the company that often included not just the employees but also their real family members. One big annual event, the organization of which involved all sections in ZIA, was to have a 'weekend' outing for all in the company and their family members. They had had this annual feast for years now, when all would travel together in hired tour busses to an up-country or a seaside resort, stayed in the same hotel and participated in all sorts of fantastically ridiculous festivities, such as beach volleyball, party games, dinner, and of course karaoke and all. Khun Fons had made a point of attending this one annual event every year. He also tried not to miss other smaller social events, if his agenda would allow.

He couldn't imagine such a thing in the Netherlands. Well, it wouldn't work like it did here, he gathered. He was glad Adriaan thought of it and intended to continue this wacky tradition as long as it served its purpose to make his staff felt at home with ZIA.

\section{The 'golden pair' in a 'corner'}

But home was also where ZIA business must thrive. Karaoke and congeniality aside, everybody at ZIA must also learn to do business the ZIA way. And the ZIA way could certainly not be the Thai way, because ZIA's target market comprised those multinationals that sought Western technical and project management capability. In this high- 
technology sector of industrial automation, the Thai had yet to catch up in both technological advance and project management proficiency. Because of the heavily computerized and rapidly advancing technologies associated with modern-day industrial automation, coupled with the huge scale and scope of a typical industrial automation project handled by ZIA - such as a modern regional factory for companies the like of Nestlé, Heineken, Unilever, or Makro - Western technical expertise and systematic project management could still not be matched by any Thai counterpart. And ZIA had to drive this fact home to its clients. This constituted its key selling point.

Again, Khun Adriaan came up with a strategy he nicknamed 'the golden pair' - a team composing of one Dutch and one Thai project managers. This combination team was found to be very effective to gain trust and respect from its clients, as the team represented 'the best of both cultures,' according to Khun Adriaan. The golden pair customer relation continued to these days, because Khun Fons knew that,

\footnotetext{
The best sale situation that you can have is when you can walk in with two people - a foreigner and a Thai. So you have the Thai face and the farang face. The Thai face to bring the Thai relationship and the farang face to bring in the fact that we are selling foreign here and that we are a good foreign company. This is the golden pair. For a big important project, we always do that.
}

Take heed, though, that the emphasis was on being 'a good foreign company'. As technically competent as the Thai could be, they were not made to be organized, systematic and punctual and these were the key to successful large-scale industrial automation projects. Thus it was always the Dutch who took the lead in this golden pair. Khun Fons insisted that it was so, because,

\begin{abstract}
We believe that to run a dynamic and an aggressive organization that we need to be in our industry, requires a mix of expats and Thai people together in one corner. And what's this corner important for? First of all, it's to bring this kind of mentality into our Thai employee base but also to monitor the quality of the work we do. And to make sure that people meet deadlines. Because even though I think that the Thai people understand the system and the deadline, we still need to keep that corner. Because if we take away that corner, the organization will easily slide back into the Thai style organization.
\end{abstract}

Both Khun Ralf and Khun Danny managed their team with this 'golden pair in a corner' philosophy. Khun Somphob and a few other senior Thai managers took on the task of echoing this message to other Thai staffs. So the Thai staffs knew to observe this corner well. Those that did not agree and refused to stay in the corner would eventually leave any way, like the one sales manager who found his corner with Khun Danny to be too suffocating that he eventually left of his own accord. Those who stayed, though, knew to take to the corner that was the heart and soul of ZIA's business principle, whenever they were asked to do so.

This was a family, but it was a family with a strong Dutch discipline.

\title{
Dutch at heart, Thai up front
}

As such, Khun Danny's characterization of ZIA was right on. ZIA was 'a Thai company with foreign management', meaning it was a company operating in Thailand in a Dutch style. Just as Khun Danny went on to explain, ZIA would 'try to be as Thai and local as possible' as long as that could be contained and would serve a purpose. At its heart 
though, ZIA was Dutch through and through, in the way it conducted its business and managed its people. Whatever elements in the company that resembled Thai traits, they were there by design, to make the Thai feel willing and able to work the Dutch way.

Let's take a look at how this 'Dutch at heart, Thai up front' manoeuvre was carried out at ZIA.

\title{
OK, you are the choir, we are the conductor
}

It always began in the beginning, of course, that is, right at recruitment. For starters, ZIA aimed to attract those people whose preference and personality were already compatible with its business style. Khun Nicki was a classic case. She heard of ZIA from a friend who had joined the company and liked what she saw. The friend related her experience to Khun Nicki, knowing how this environment would appeal to her friend's modern spirit. Khun Nicki gathered up the audacity to set up a meeting with Khun Fons and convinced him that there was room for a new position of a marketing executive and she was just the right person to take up the challenge. Khun Fons took her on the spot, a decision quite uncommon in the Thai human resource tradition, where at least two rounds of interview were a norm and above all never for a position not already there but conjured up out of sheer instinct. But that was how ZIA took on people, in a manner that was compatible with the type of people the company looked for. In a sense, ZIA sent out a signal to attract just the right people.

Once they were drawn in, other signals were then constantly transmitted to let all know how things were to be conducted. Most strikingly, even the 'familial' spirit was given a nice twist to make it clear that this was not just a family, but it was a family with a 'Dutch' manner of rearing. Khun Fons put it this way.

\begin{abstract}
So you have to create an environment in which your people feel comfortable. You have to show them that they can trust you, that you'll be there for them, that you try to understand them. And next to that, you have to create a box to say what you allow in that and then outside the box it's not allowed. Because if you make it too loose then they will walk away with you. It's like in a family, where the parents allow things with children. The children, they are good children, but every time they think they can do this much, they will always try another step and another step. So they have to have room and responsibilities where they can do things but there have to be clear rules about what can and what cannot.
\end{abstract}

Thus, ZIA was a family with an obvious Dutch style of upbringing. This family metaphor was quite apt, Khun Fons reflected. In an average Dutch family, Khun Fons knew how children learned everyday life disciplines such as eating properly at the table, going to bed at a regular time, having their own space for which they were also responsible, working and playing when appropriate, and so on. In a normal Thai household, by contrast, he noticed that disciplines were not to be set in a regular rhythm; instead they were announced by parents whenever they felt the need and then observed by children whenever they were summoned to. Indeed, Thai children conducted their lives without strict living disciplines. He had seen how they ate when and where they were hungry and went to bed only when they were tired. Every space in a 
Thai household belonged virtually to all. Working and playing went mostly hand in hand.

But if ZIA had to be a family, it had to be one with a Dutch parental mindset. ZIA, as a choir, was one with a Dutch conductor leading his singers to sing the Dutch style. They might be singing a Thai or English song, or what have you, but they would still sing it all following a Dutch musical arrangement.

\section{And here's the score}

So, ZIA was a family with members from many origins, Dutch, Thai, Belgium, Chinese and so on, but it certainly had Dutch parents and a good set of highly organized, well disciplined Dutch business principles. Khun Somphob recalled how this was one of the first 'score' he needed to master when he started with ZIA. He had worked for ten years doing practically the same work he was supposed to be doing at ZIA - implementing industrial automation projects for business clients of the Thai company he previously worked for. But mind, how completely different things had to be done here. In his former job, he only had to care about completing the projects as agreed with the clients. That was it - they just had to get it done, one day, in good time. The only other two things to worry about were to keep the costs low and the clients happy.

But when he started at ZIA, the Dutch were so fussy about having a detailed, accurate project plan right from the start. He couldn't see the point. At his former company, the plan was there only to let the clients have some idea of what they would get and to give the engineers a general guideline of what were to be done. They never had to seriously think through how many man-hours would be needed for this and that stage, or when this and that were to be started and finished. Actually, for Thai companies, the less you can book your man-hours, the better, because Thai clients never wanted to pay too much for mere 'labour'. You put your charges on something concrete like a modern machinery or equipment. Also, with Thai clients, you told them as you went along how things were and what were to come, no exact timeline had to be so carefully laid out and meticulously followed. But no, it was not so with the Dutch. They wanted to put things exactly how they were. They had a rule for all estimates what margin for what products, how many man-hours for what job, what came first and what came next, and when and where and how.

And to top it all, they took that blueprint religiously. What a nightmare! Khun Somphob learned soon enough that if it was estimated for him to spend forty manhours for a particular job, he had to stick to it and get things done within that estimate. The blueprint was not just a conjecture of the tune to hum; it was an exact score of all the little notes he had to reproduce, at precisely the right moment and in the directed tone and tempo. It's not just a matter of working to complete a project, but to complete it as laid out in the plan. This was a totally strange way of doing things for Khun Somphob and most Thai engineers.

But Khun Somphob was gradually made to see the point when he saw how his Dutch bosses and colleagues derived the bottom lines of ZIA projects. They actually 
used this score as the basis to see how well a project was carried out. If a project was planned well, it should be completed as planned and profits gained precisely as projected - a happy ending. If the project did not go as planned, then it was either the plan was flawed or the work was inefficient, either way you would know what to improve. Khun Somphob learned to accept and even appreciate this philosophy and adjusted his working attitude and style accordingly. In a way, he came to like it because this way he could easily see when he did a good job, which was a rewarding feeling to have. Now he was one of the main channels to translate this project management style to the new recruits. Some of his 'nong nong' would be like him and adjust themselves; some would not and eventually leave because they didn't fancy the pressure that came with it.

On the flip side, Khun Ralf and Khun Danny found out from experience working with the Thai how important it was to instil in them a new attitude to carry out engineering and sale projects. Khun Ralf recalled how he eventually learned that his new recruits 'who have no experience with managing the project, would start doing before they start thinking, approaching a new project by just start doing things and not thinking things through at first'. More, they also expected him, as their boss, to act like their professors and have ready answers to all their work problems. They only wanted to know the solutions to carry out. Khun Ralf's main work in moulding these young engineers was thus to let their work experience taught them that real-world engineering work was actually all about finding solutions to new problems and project management needed good planning before any action could be taken.

Khun Danny, for his part, found it crucial to have an 'attitude adjustment' scheme to convince his sale team of a more egalitarian Dutch attitude towards sale management.

Sale task is to offer advice, offer solutions to a customer. For the salesperson, the first feeling about the customer is the customer is always sky-high, always the boss. And the customer can speak down always to the sales, saying this and that. And the majority of the Thai people, Thai sales personnel, they don't know how to handle that. So what I always say to them is you need to 'educate' your customers. You have to educate them what you are doing, what you're not doing and what they can expect from you and what you expect from them. It's a two-way communication. So, the salesperson also chooses the customer. If you are a salesperson and you see this customer is not someone you can work with, he's not polite to you or you don't feel comfortable with, then don't do it. Give that opportunity to other people, maybe they can do it. So I have to emphasize to the Thai staff, you don't need to be scared of your customers.

In that way, Khun Danny handed a totally new sale 'score' for his Thai sale team to sing.

So, all at ZIA knew that if you wanted to be a part this choir, you had to keep to the score and sing to the conductor's direction.

\section{And the chameleon keeps on changing?}

Thus, even now, when Khun Adriaan was no longer a presence at ZIA, his imprints were still going strong. You could feel it in the air. You could detect it when you talk to the people there - how much they valued the familial spirit and how willing and able 
they were to abide by the Dutch business discipline. Khun Fons looked around him today. How different ZIA was from less than a decade ago, when they were a sheer family of seven. Now ZIA was more a big clan that kept growing. He really wondered how long things would stay the same. Would this chameleon kept transforming itself but still keeping its original spirit in tact as it moved on?

\subsection{Lessons from the cultural chameleon}

ZIA as a cultural chameleon illuminates two crucial elements of the grounded theory of XCQ amid intricate cultural webs. First the story of ZIA provides vivid evidence of how a cross-cultural condition is a tangle of intricate cultural webs forever being spun. Second, the unique chameleon like ability - negative capability - detectable in the narrative of ZIA underscores a critical property of cross-cultural intelligence or XCQ.

\section{Variant cross-cultural modes within intricate cultural webs}

As described in Chapter 4, a cross-cultural condition is in essence a tangle of myriad intricate webs of many individual cultural units and composite groups. The case of ZIA illustrates how a tangle of intricate cultural webs is woven - what composite shapes and forms could characterize it and how this spinning process moves forward in space and time.

In short, the case of ZIA suggests that in a particular set of intricate cultural webs, variant modes of cultural crossing co-exist in a collectively exhaustive but not mutually exclusive manner and with a dynamic yet not necessarily linear progress along space and time.

\section{Collectively exhaustive but not mutually exclusive}

To recall, the four representative modes of cross-cultural condition are: clash, reciprocal, unification, and variation. In any cultural unit (individual or group) and crosscultural condition, at a point in time and space, there must exist at least one of these modes, a natural consequence of the 'crossing' of cultures. Yet, it is not necessary that only one of the four modes exist, again even at a single point in time and space. By its very nature, a cross-cultural condition comprises many individual cultural units and composite cultural groups. At the simplest level of analysis, each of these myriad individual cultural units and composite cultural groups must manifest at least one, often more, cross-cultural modes. In the end, intricate cultural webs are a composite of these co-existing modes.

To illustrate, at ZIA, we witness individual cultural units of many variety. Khun Adriaan, the founder, was a unique cultural chameleon: most of those who met or knew him would emphasize more how they felt Khun Adriaan understood them but rarely attempt to identify him with any particular culture. Khun Adriaan exercised his chameleon like ability less to blend in than to, first, communicate his intention and second, to create a comfortable cross-cultural atmosphere. By comparison, Khun Danny was more of a cultural hybrid, that is a Belgium-Dutch composite, through birth 
and then training. Khun Danny observed that his business conduct was more Dutch than Belgian, a result of his affinity with the effectiveness of Dutch business principles when compared to the more relaxed Belgian attitude. Yet, he also manifested an affinity towards Belgian cultural elements, such as the social and congenial spirit - one of the reasons why he felt at home with Khun Fons who came from 'near where [he]

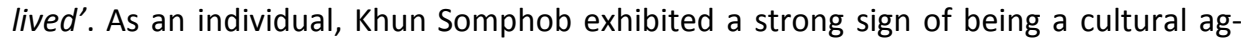
gregate, combining and making the best of both worlds, that is Dutch business ethic with Thai social spirit, in his role as the intermediary within ZIA. He treated his team with a 'pi nong' or familial spirit, yet his messages to them, both in words and deeds, about how to perform their job were essentially Dutch.

Thus, within the tangle webs of ZIA, individual cultural units characterized by diverse cross-cultural modes did co-exist. This was the simplest co-existence. Further, even within one individual cultural unit, more than one cross-cultural mode could simultaneously exist. Take Khun Fons as an excellent example. He functioned alternately as a cultural hybrid and a cultural chameleon. Reflecting on his own identity, Khun Fons proclaimed: 'once you have known a new culture, you cannot go back to be your own self anymore.' As a husband of a Thai wife and a father of a Dutch-Thai offspring, he found himself embracing a few aspects of both cultures in his daily life. Yet, as a top executive of a 'Thai company with a Dutch management', his professional identity was more of a chameleon, in the same fashion as Khun Adriaan, his business mentor.

Next, it is conceivable to extend the same analysis to the level of composite cultural groups. Let us attempt this with a look at ZIA. First, ZIA as a company exhibited a strong characteristic of a cultural chameleon. Thus, ZIA's most predominant crosscultural mode was that of the variation mode: it was a Dutch company that camouflaged itself according to whatever environment it operated in. To its Thai employees, it camouflaged its strong Dutch disciplinary spirit with Thai social congeniality. However, to its (mostly Western) clients, it let its Dutch expertise shine through, in the Dutch manner of project and client management. Within ZIA, though, we could detect a few other cross-cultural modes among its internal groups and sections. A clear clashing mode occurred occasionally, as with the case of the sole sale manager who resigned after finding that he could never be compatible with Khun Danny's 'corner' of conducting sales. Elements of a reciprocal mode could be discerned in how the Thai familial spirit of the company was applied to its social conduct, while the Dutch business ethic and discipline to its business operation.

The portrait of ZIA was one of a tangle of intricate cultural webs, with many coexisting cross-cultural modes at many levels of analysis. Looking back to the two earlier cases of $A D T$ and CDW, the same analytical exercise would yield yet two more portraits of the same concept of intricate cultural webs - a constant comparison exercise that inspired the emergence of the last conceptualization in this study, that of cross-cultural heuristic or a practical way to untangle these webs, as explained in the previous chapter. 


\section{Forever proceeding but not linearly progressing}

Also, when the narrative of ZIA was compared to that of the two earlier cases, the coexistence of variant cross-cultural modes suggested that although these modes are constantly evolving, responding to changes in the context, their evolution hardly proceeds in a linear fashion. Although our scientific mindset may motivate us to deductively line up the four modes as relating to each other in a neat successive manner, the real world proves to be not as orderly as our logic would have it. For example, a rational conceptualization of the relationship between the four modes would be to hypothesize that a cross-cultural condition tends to start with the clashing mode and then advances further to the reciprocal mode, which is then succeeded by either the unification or variation mode.

As logical as that hypothesis may sound, in reality, there seems to be no precise fashion to predict how the four cross-cultural modes evolve, in relation to each other. ZIA started off with a strong element of the variation mode, in the character and intention of its two founders. CDW, the odd-eyed cat, by comparison, emerged out of a strong ingredient for a reciprocal mode, also in the character and intention of its two co-founders. ADT, the first narrative, by contrast, experienced several competing modes from the start, a little of clashing, reciprocal and unification simultaneously. As ZIA and CDW evolved, their initial cross-cultural modes strengthened while other cross-cultural modes did surface at various occasions. The dynamic of variant crosscultural modes at ADT, however, seemed to be fluctuating still among the initial competing modes and only time can tell whether any one of them will eventually emerge as a predominant mode.

As such, it appears that context, and not logic, stands as a better indicator of how cross-cultural condition evolves. Actors in a cross-cultural condition respond to the specific context of a situation they are operating in and their interactive responses collectively define the dynamic of the forever evolving cross-cultural co-existing modes. At one point in space and time, one steady-state mode may prevail as the predominant mode, yet this state is also evolving with context. The significant of context that eventually affects the exercise of variant cross-cultural instruments thus constitute part of the cross-cultural heuristic as already expounded.

\section{Negative capability: how chameleons mesmerize friends and foes}

The most intriguing step in the proposed cross-cultural heuristic is probably its very first step - exercising negative capability. ZIA provides evidence of the application of this particular capability. But before that analysis, a brief recapitulation and further elaboration of the concept of negative capability only briefly introduced in the preceding chapter is now in order. 
The term 'negative capability' was coined by an English poet of the romantic period, John Keats. ${ }^{32}$ Keats, besides being acclaimed for his poetry, also wrote a number of inspiring letters and the term negative capability originated in one such letters. Keats used this term in the following passage in a letter he wrote in 1818 to his two brothers:

[S] everal things dovetailed in my mind, \& at once it struck me, what quality went to form a Man of Achievement especially in literature \& which Shakespeare possessed so enormously - I mean Negative Capability, that is when a man is capable of being in uncertainties, Mysteries, doubts without any irritable reaching after fact \& reason $[\ldots]$.

(Keats and Forman 2004: 57)

There Keats argued that what distinguished great characters, such as great writers, was their ability to 'negate' or eliminate their own identities to the extent that their character became a clean slate that could assume the identities of other entities and understand them so well as to excel in whatever they set out to achieve, such as writing so convincingly and realistically about those entities. Negative capability is in essence the ability to negate or suspend oneself, so to speak. ${ }^{33}$ The ability to suspend one's identity is the key to a complete reaching out to the unknown - the 'uncertainties, Mysteries, doubts' without the burden of 'any irritable reaching after fact \& reason' that would breed assumptions and their resulting preconceptions. Negative capability allows one to have an open mind that is receptive to an understanding that is free from contamination and hence is the state of mind that has a greater chance of leading to a robust understanding of the unfamiliar and the unknown.

For the narrative of ZIA, though, Keats' metaphor for an individual with the faculty of negative capability was interestingly that of a chameleon. This he expounded again in a letter to Richard Woodhouse in 1818, in which he discussed how he would characterize a good poet as being chameleon like,

A Poet is the most unpoetical of anything in existence; because he has no Identity - he is continually in for - and filling some other Body - The Sun, the Moon, the Sea and Men and Women who are creatures of impulse are poetical and have about them an unchangeable attribute - the poet has none; no identity - he is certainly the most unpoetical of all God's Creatures.

(Keats and Forman 2004: 210)

The term 'chameleon poet' has since represented Keats' characterization of an artist whose talent in accurately re-present other entities rests in his or her ability to assume infinite identities of whatever entity he or she is reaching out to portray. Negative ca-

\footnotetext{
32 Here again, it should be pointed out that my previous training in English literature has definitely influenced why the term emerged to denote this concept and how it is associated with the use of the chameleon metaphor.

${ }^{33}$ The term 'negative' as used by Keats is easily mistaken to denote something bad (as opposed to 'positive' which denotes something good). Such is definitely not the case. Rather, the adjective 'negative' in this terminology has its root in the verb 'to negate' or to render ineffective. In this sense, 'to negate' is the opposite of 'to posit' or to take as valid, which is the root of the term positive science. In both uses of the term positive and negative, no value judgment is actually associated with the terms - negative capability does not denote a bad faculty, just as positive science does not denote a better philosophy (as observed earlier in Chapter 2).
} 
pability enables a human chameleon to develop an empathy with the subject of his or her attention. It is also the core capability of a cultural chameleon like Khun Adriaan or Khun Fons, as an individual cultural unit, or ZIA, as a composite cultural group.

To recall, chameleons change their skin colours (an adaptive response) to achieve one or more of the three biological purposes - the three C's of camouflage, comfort and communicate - that is: 1 ) to blend in as a technique to hide from predators or to mislead preys; 2 ) to induce comfort vis-à-vis diverse environment; or 3) to communicate moods or desires. In most cross-cultural interaction, the first chameleon effect is fairly common: witness the popularity of cultural guidebooks, whose very first instructions often detail what specific outward behavioural gestures are to be observed and 'mimicked' in order to simply blend in and portray goodwill. Most cultural guidebooks about the Thai culture, for example, always teach foreigners how to 'wai' (a rough equivalent of the Western handshake), or warn them never to put their feet higher than the ground, and so on. Most cultural guidebooks about the Dutch culture, by comparison, invariably point out how punctuality is sacred to the Dutch, or instruct foreigners that a vital etiquette for a Dutch telephone conversation is to first clearly identify yourself, and so forth. In this narrative of ZIA, Khun Fons illustrated well how his response to the karaoke fervour was a gesture of 'blending in'. Deep down, he would never enjoy the festivity in the same way many Thais would, but he still appreciated why they did so and he went along with it - he assumed the 'karaoke colour' for the occasion, so to speak.

Yet, just like natural chameleons, cultural chameleons also go beyond the blending in effect and use their negative capability to achieve an adaptive response that induces comfort or communicates, or both. This was also illustrated well in the narrative of ZIA. For example, Khun Somphob recalled how Khun Fons and Khun Ralf adjusted their articulation of English to accommodate the Thai's unfamiliarity with the language: 'So, when they speak to us, they try to make it easier for us too. They know that we are not that great in English, so they try to speak slowly and listen well. But for some new expats, oh - they can speak so fast and are not used to Thai English, then, it's a bit more difficul'. At an organizational level, ZIA used its negative capability to change its 'organizational colour' to create comfort as well as communicate. The deliberate social functions were by design an adaptive response to the Thai's fondness for felicity and congeniality, whereas the ZIA office in the Netherlands did not have the same tradition. The social functions at the Thai office were in this sense a mechanism to induce comfort befitting the Thai cultural environment. Another chameleon like exercise was the intentional familial and modern attitude that ZIA management carefully crafted as a signal to its existing and potential employees to communicate its organizational culture in a way that would appeal to the kind of people it sought. Yet, this familial spirit, as discussed earlier, was in fact Dutch by its very nature - again, another deft chameleon effect. 


\subsection{Final words}

\section{Khun Adriaan}

With the Thai, there is a 'fine line' governing an acceptable level of friendliness. So, we have to be very careful in dealing with Thais, to stay within the acceptable level of friendliness because if we step over this, the situation can turn quite ugly.

But learning how to 'read' the non-verbal communication of the Thais is difficult. For example, Thais always appear friendly but it's never clear what level and degree of friendliness is involved.

\section{Khun Fons}

If you don't adjust yourself to this environment then you won't last very long. Because it's not that you can ask the Thai people in this country and in this organization to change. We have to accept we are in Thailand. I see this as something that is normal and inevitable.

Now if I go back to the Netherlands, I do adapt back but I also find a lot of things that are difficult in my own culture.

\section{Khun Ralf}

As an expatriate, though, in the end you will belong nowhere. We live abroad for a very long time and when we go back to the Netherlands it'll be very difficult. You'll never really fit into the local community anymore. But then even if I've been living here for ten, twenty years, I'll still be a foreigner. So at the end of the day, you'll be in-between those worlds.

I don't think it's a particularly bad thing, though.

\section{Khun Danny}

The most important things that I said before and I will repeat, is to keep yourself ready and accept things, accept that it can go in another way. That you need to do in another way than what you've learned to do, maybe, in the biggest company in the world, for the past ten years or so. It is here different.

\section{Khun Somphob}

I mean you should not bias towards either side. It's a balance you have to make. This is what I try to pass on to my people, to 'nong nong'.

I try to tell them, it's not always that we employees can have whatever we want, because the company also has its own wants. I ask them to try to put themselves in the management' and the owners' shoes and ask, OK if you were them, what would you do? A lot of time this helps them to see that we cannot always get what we want.

\section{Khun Nicki}

Well, the Dutch can be direct, I agree, and I think if I get direct comment on critical, big issues, I might feel hurt too. Luckily, so far it hasn't happened to me yet.

Mostly it's nothing so serious, so so far it's $O K$.

\section{回}

S A CASE, ZIA ILLUSTRATEd THAT NEGATIVE CAPABILITY PROVED TO BE THE KEY TO A SUCCESSFUL adaptive response - one needs to first know the nature of what one sets out to respond to in order to create the right response. Cultural chameleons are masters of negative capability, yet this key capability can be employed in all cross-cultural condition, in varying degrees, by any cultural unit or group. How do we develop negative 
capability? That, I believe, is the next frontier in cross-cultural research, an observation that will be reflected upon in the following concluding chapter.

$.333<\rightarrow 388$. 



\section{Chapter 8}

\section{THE ROAD AHEAD}

\section{The journey is the destination.}

Action is eloquence.

William Shakespeare

Coriolanus (III, ii, 75-77).

1605-1608

. 3334 $\rightarrow 88$.

What I call the beginning is often the end And to make an end is to make a beginning.

The end is where I start from.

\section{$3334 \rightarrow 88$. .}

'Would you tell me, please, which way I ought to go from here?'

'That depends a good deal on where you want to get to,' said the Cat. 'I don't much care where -' said Alice.

'Then it doesn't matter which way you go,' said the Cat. '- so long as I get SOMEWHERE,' Alice added as an explanation.

'Oh, you're sure to do that,' said the Cat, 'if you only walk long enough.'

Lewis Carroll (Charles Lutwidge Dodgson) Alice's Adventures in Wonderland 
Oftentimes in this whole journey, I felt very much like Alice in Wonderland. I would be walking and wanting to know which way to go next. In the end, the cat had a point: in my chosen 'Wonderland' of research choices - both topic and approach, it didn't really matter which way I took, as long as I kept walking in a purposeful and curious manner. Now, I've walked long enough and finally got somewhere. Then again, that somewhere felt strangely like yet another beginning, and the whole journey was turning out to be as much a destination as it was an expedition. If this makes it sounds like a merry-go-round, it is at least a curious one, because it has been and looks to be, to me, a rather appealing and productive merry-go-round of research, if there ever is such a one.

Looking back to my earlier encounters with foreign cultures, I remembered how irritated I felt when my American professors of literary criticism kept pointing out to me to 'quote your sources' - meaning I could not simply talk about ideas without saying 'who' originated them. As a Thai, my intellectual orientation had been more towards the concepts themselves than the persons behind them. We Thais identify theories and ideas by their names, not by the names of their creators. I had not been trained to pay attention to authorship and proper quotation, in the Western style, that is. It took me a long time to get into the Western mode of proper referencing and cataloguing of ideas and theories by their authors. You could say that this reflects the difference meanings different cultures have of 'knowledge' and 'knowing'. While the West are more individualistic in their view of knowledge - knowledge is a product, a pride and joy of an individual - the East see knowledge more as a collective achievement of a community, and its significance is tied more to its quality rather than to an individual. A nice little interpretation (which I actually should cite some references here), at the very least, to explain why I was irritated with the prominence of authorship in Western academic community.

Even now, I still have an occasional tendency to be more oriented to the ideas themselves rather than to their authors, although I am by now well trained to exercise the academic referencing practice of the Western tradition.

And if I were to be given a choice, what would I prefer? Really, it depends. I see the merits in both orientations and I would simply choose one or the other, one case at a time. To get this doctoral degree and to be heard in Western academic community, I will respect the prominence of authorship. At times, I even enjoy the fruits of this tradition. Certainly now as a teacher, I am the one who annoy my students when I keep asking them to 'cite your sources!' Yet, when I am really absorbed in any intellectual endeavour, I tend to be more intrigued by the ideas than by their association with any living creature, great or small.

I can see that this study has instilled in me a tendency to relate the thoughts, feelings and acts I witness to the very theory I articulate here. This is indeed a destination that looks to be a life-long journey.

\section{'CURIOUSER and curiouser!'}


T HIS IS A CONCLUSION TO MARK A COMPLETION BUT NOT THE END. THE FRUITS OF THIS STUDY signify a few noteworthy contributions to the research domain of culture and learning in organizations and open up attractive prospects for future research. At the same time, I must acknowledge a number of limitations that have constrained this study; yet, in retrospect, they also offer humble lessons that will help strengthen future research effort even further. These contributions, opportunities and limitations thus constitute as much an end as a beginning.

In that spirit, my concluding deliberation will first take stock of the current standing before looking forward as well as backward. In taking stock, I will review the major theoretical, methodological and practical contributions that constitute the key outcome of this study. In looking forward, I will deliberate on future research prospects to advance the findings further. My deliberation will then end with one last look backward, when I apply the principle of reflexivity to ponder on limitations that constrained this study, the resulting lessons learned in the course of the investigation and how they can contribute to advance future investigation.

As popular culture will have it, this concluding chapter is a kind of 'back to the future' experience where the journey is indeed the destination.

\subsection{Taking stock: contributions}

Stocktaking of the outcome of this study reveals three major areas to which its findings can claim to make notable contributions: theory, methodology, and practice. On the whole, this study - its findings as well as approach - represents a fresh view of cultural force that entails distinct theoretical, methodological and managerial implications for research effort and organizational practice relating to the theme of culture and learning in organizations in general, and that of cultural intelligence in particular.

\section{Theoretical contributions}

Theoretical contributions constitute the most important outcome of this study. It was the single key objective from the start - to build theory on culture and learning in organizations using interpretive grounded theory in conjunction with qualitative case study research (Corbin and Strauss 1990, Dey 1999, Douglas 2003a, Douglas 2003b, Eisenhardt 1989, Geertz 1993, Glaser 1978, Glaser and Strauss 1967, Goulding 1998, Goulding 2002, Locke 2001, Martin and Turner 1986, Miles and Huberman 1994, Partington 2000, Simons 1996; Simons 2009, Stake 1995; Stake 2005; Stake and Trumbull 1982, Strauss and Corbin 1990, Strauss and Corbin 1994, Suddaby 2006, Turner 1983, Walker and Myrick 2006, Weick 1989). The proposed grounded theory by the name of cross-cultural intelligence amid intricate cultural webs - elucidated in detail in Chapter 4 and 6 , and illustrated via thick descriptive narratives of Chapter 3, 5, and $7-$ is the main theoretical contribution that encompasses a number of new perspectives on the theme of culture and learning in organizations, from the very holistic image of culture 
as intricate webs of meanings to the concept of cross-cultural heuristic that epitomizes the fundamental tenet of XCQ in action.

\section{Intricate cultural webs: a holistic view of culture}

First, this study's findings entail an expansion of the hitherto undeservingly less known conceptualization of the concept of culture. Although the image of culture as interweaving webs of meanings has been vibrantly proposed by Geertz (1993) over two decades ago, this powerful image of culture has been unduly overshadowed by the more popular, and much popularized, value-dimensional view. The dimensional model of culture as a highly compartmentalized and organized system of values or other cognitive constructs was earlier pioneered by notable anthropologies (Inkeles and Levinson 1969, Kluckhohn 1951, Kluckhohn and Strodtbeck 1961, Murdock and Provost 1973, Rokeach 1968, Rokeach 1973, Triandis 1982), later championed and fortified by Hofstede (1980, 2001, 2005), and overwhelmingly extended in the burgeoning dimensional studies of the recent decades (Bardi 2001, House, Hanges, Javidan, Dorfman, and Gupta 2004, Inglehart 2007, Inglehart, Basanez, Diez-Medrano, Halman, and Luijkx 2004, Kirkman, Lowe, and Gibson 2006, Maznevski, Gomez, DiStefano, Noorderhaven, and Wu 2002, Peterson and Castro 2006, Sackmann 1991, Schwartz 1999, Schwartz 1992, Schwartz and Bilsky 1987, Schwartz and Sagiv 1995, Smith 2004, Smith, Peterson, and Thomas 2008, Søndergaard 1994, Trompenaars and Hampden-Turner 1998, Usunier 1998, Welzel 2007). This dimensional view of culture has attracted tremendous attention and consequently dominated cultural studies for many decades. Although highly valuable and informative, such view of culture has its limitations, as elaborated as length in Chapter 4. Most significantly, such systematic and compartmentalized view of culture, while highly compatible with quantitative and reductionist approach to cultural studies, makes it impossible to investigate culture and its forces under a more naturalistic and holistic light - a perspective also needed if we were to extend our investigation beyond the static and compartmentalized effect of cultural forces. Here is where a completely different image of culture comes of use. This study, in essence, revitalizes such an image of culture, one proposed decades ago by Geertz (1993) and emerged as one of the two core conceptual categories in the grounded theory that resulted from the analysis of this study's evidence - the image of intricate cultural webs.

Culture is conceptualized in the proposed theory as a shared way of being and sense-making that is unique to a distinct group of people and can distinguish that group from their counterparts. Members of a culture are cultural units and these units must operate in numerous cross-cultural platforms. The first core category of intricate cultural webs then denotes the property that characterizes first cultural unit (an entity), second cross-cultural interface (a process) and third, cross-cultural platform (a condition or context) in which this process transpires. Intricate cultural webs are the fundamental property of any cultural unit because all cultural units (even single individuals) invariably belong to or are made up of more than one cultures, and their 
property can be metaphorically characterized as intricate cultural webs that are made up of diverse cultural traits that simultaneously influence the operation of those cultural units. Thus, 'cultural identity' refers to the combined cultural forces within a cultural unit, whether an individual or any type of groups - be it team, organization, nation, or others. Finally, cross-cultural interface is a social process that transpires in a cross-cultural platform - a social setting or context that entails two or more competing cultures. Again, intricate cultural webs also characterize cross-cultural condition and the social process that transpires within the context of that condition.

The image of culture as intricate cultural webs thus provides a holistic lens to look at culture and all facets relating to it - the unit, the process and its context - in a simultaneous, interrelated fashion. This holistic perspective on culture thus offers a complementary approach to the well-known dimensional, atomistic view of culture that focuses on investigating its disparate elements. Together, the two approaches should enrich our effort to investigate culture and its forces even further.

\section{Beyond clashing: an expanded view of cross-cultural condition}

Second, this study's finding also entails a signification expansion of a prior concept related to culture, namely that of cross-cultural condition. To review this contribution, a brief clarification of the term 'cross-cultural' as used and meant in this study must be recapped because this term has a specific meaning and serves an imperative choice in the theorized taxonomy of cross-cultural condition. In brief, the term 'cross-cultural' here denotes a meaning that goes beyond traditional connotation of mere comparative examination. The need to go beyond simple comparative perspective has recently been put forward by many prominent cross-cultural scholars (Boyacigiller, Kleinberg, Phillips, and Sackmann 2004, Earley 2006, Jackson and Aycan 2001, Leung, Bhagat, Buchan, Erez, and Gibson 2005, Soderberg and Holden 2002, Tjosvold and Leung 2003, Yeganeh, Su, and Chrysostome 2004, Zhu and Ulijn 2005). Following this and the study's evidence, the meaning of the term 'cross-cultural' is expanded to designate issues involving different cultures not only from a comparative angel, but also, and most specifically, from an interactive viewpoint, namely at their interface or 'crossing'. Thus, 'cross-cultural' signifies not only the traditional comparative treatment, but also, and even more importantly, the interaction facet of a cross-cultural phenomenon.

Ever since the landmark publication of Hofstede's Culture's Consequences (Hofstede 1980) and Huntington's The Clash of Civilizations? (Huntington 1993), much of the world's and academic attention on cross-cultural matters has concentrated heavily, if not exclusively, on the clashing of cultural differences. Indeed culture differs, and clashing is the most dramatic consequence of cross-cultural interaction, thereby justifying the enormous attention paid to the phenomenon. Thus, despite the quest for peaceful and constructive co-operation that underlies cross-cultural investigation, we have ironically paid most of our attention to the potentially damaging aspect of the process. No doubt, greater insights into the clashing effect of cultures have helped us deal better with its consequences. Yet, perhaps the time has come to expand our view 
of cross-cultural interactions beyond the clashing facet. Such an expanded view will not only further strengthen our understanding but also better equip us with richer alternatives in exploring and exploiting cross-cultural interfaces. This study contributes one such expanded view of cross-cultural taxonomy.

The theorized taxonomy of possible conditions for cultural crossing naturally includes the clashing mode but also extends beyond that. The taxonomy suggests four distinct modes of cultural crossing: clashing, reciprocal, unification and variation. The four modes, while distinct from each other in their characterization and implication, are found to be collectively exhaustive, yet not mutually exclusive. These modes can and do co-exist, although one may be more visible than others in a particular crosscultural context. The four modes maintain an inter-related dynamic path that is forever proceeding, yet not linearly progressing. The theorized taxonomy and its dynamics reveal several insights that may have escaped investigation in the past and provide a new perspective and venue to explore cross-cultural condition further and beyond the well-explored 'clashing' mode. This taxonomy is elaborated in detail in Chapter 6 to emphasize its most significant message - the great sophistication embedded in a crosscultural condition that compels us to move beyond our fixation on the clashing consequences.

\section{Cross-Cultural Intelligence (XCQ): a qualitative view of $C Q$}

To reiterate, the term 'cross-cultural' has definitive significance in the theory proposed in this study because it accentuates the very focus of its main finding, a grounded theory on cross-cultural intelligence (XCQ), of which the above taxonomy serves an integral basis. The grounded theory of cross-cultural intelligence amid intricate cultural webs constitutes the third and integral theoretical contribution of this study.

Cross-cultural intelligence or XCQ is a capability and strategy that is needed for a cultural unit (individual or composite group) to handle the complex intricate cultural webs that characterize both its own cultural identity and the nature of any crosscultural situation that the unit must operate in. The emphasis here is on the significance of 'cross' - hence cross-cultural intelligence, rather than simply cultural intelligence. The choice of the letter $X$ in the abbreviation exemplifies this emphasis, which marks a subtle distinction between the concept of XCQ and that of cultural intelligence (CQ).

The locus of XCQ is thus not in the nature of its focussed entity - namely, intelligence about culture, but in the nature of its interactive feature - that is intelligence when dealing with cross-cultural situation. Cultural intelligence, as defined by prominent theorists on the subject, is fundamentally about the ability to react effectively in an unfamiliar cultural context (Earley and Ang 2003, p. 59, Peterson 2004, p. 89, Thomas and Inkson 2003, p. 62). By comparison, cross-cultural intelligence is the ability to operate successfully in a context where the crossing of more than one culture is the salient condition. Thus, cross-cultural intelligence puts emphasis on the implications of the interaction, namely crossing, of cultural forces, rather than on the necessity to 
accommodate or adapt to one particular cultural influence. Granted, this is fundamentally a difference in focus and not in substance. The two concepts of CQ and XCQ are very much related. In the final analysis, cross-cultural intelligence is but a sub-set of cultural intelligence, since XCQ is certainly part of the ability of a cultural unit to react effectively in one particular unfamiliar cultural context, namely cross-cultural context. Chapter 6 provides a thorough elucidation of the concept of XCQ as well as its relation to the greater research theme of $C Q$, particularly on how the theorized XCQ concept and its implications contribute a set of new and complementary views and opportunities to the emerging research effort in CQ.

\section{Cross-cultural heuristic - XCQ in action}

Finally, Shakespeare's crisp expression - 'Action is eloquent. ${ }^{34}$ - well accentuates the eventual implication of this study's theoretical contribution: any endeavour motivated by the concept of XCQ must consider action as its indispensible element, that is to say, cross-cultural intelligence in action. At the heart of the tenet of XCQ in action is a simple heuristic procedure employed by cultural units in their everyday dealing with crosscultural interaction. The very nature of XCQ - as a tacit, learning-by-doing strategic capability that is deeply embedded in practice - dictates that the understanding, development, and application of it must be rooted in actual action. Investigation on and application of XCQ at any one dimensional facet alone (be it cognitive, psychological or behavioural) is of limited value at best, virtually futile at worst.

The fundamental tenet of ' $X C Q$ in action' thus exemplifies the key message of this study's theoretical contributions - that the road ahead for future application and investigation must be anchored in action which can be framed within the simple heuristic procedure elaborated at length in Chapter 6. This simple heuristic procedure, depicted in Diagram 6.3, calls for four interlinked, goal-oriented deliberations: 1) invoking negative capability to achieve an open-mind; 2 ) surveying the situation to gain an understanding of the contextual attribute and demand; 3) setting situational anchorage against desired and desirable benchmark; and 4) applying cross-cultural interaction mechanism that is compatible with the context and the identified goal. The exercise of this heuristic is in essence XCQ in action.

Action encompasses two key elements. First, it calls for an intertwining of all three dimensions of human faculty: cognitive (think), psychological (feel), and behavioural (conduct), into one single manifestation. Action is a joint, indivisible and simultaneous expression of these three dimensions. Action encompasses both verbal and non-verbal mechanics, and even the 'interaction' of the two. Action also includes interaction, reaction and inaction. Second, action as human expression is context bounded. We act as a response to a situation. In other words, an act bears a particular meaning only when interpreted against its setting. Given this premise, XCQ in action denotes action that is

\footnotetext{
${ }^{34}$ Shakespear's Coriolanus (III, ii, 75-77).
} 
both governed by and hence indicates the quality of XCQ in a cultural unit, be that individual or group.

The very property of XCQ that this study exemplifies has moved cross-cultural interaction and capability from the realm of codified knowledge to a new arena of tacit knowledge, a know-how, which comes only with actual practice. XCQ, as theorized in this study has a unique property of being recursive (feeding on itself) and reflexive (directed back to itself). The more a cultural unit uses its $X C Q$, the better that unit is at exercising it, not unlike other tacit skills such as driving, sailing, dancing, or public speaking. This dictates a direct link to persistent experience of action that needs XCQ as its driver and can be detected via the framework of the theorized cross-cultural heuristic. Thus, XCQ in action encompasses three major components: observable action (verbal and non-verbal), bounded context, and repeated practice. To understand, apply, develop and assess XCQ, we must continually observe actual action in relation to diverse realistic, naturalistic cross-cultural contexts. This is the imperative principle that must underlie all theoretical, methodological and practical matters in relation to XCQ.

\section{Methodological contributions}

A survey of major review pieces on research efforts in cross-cultural management reveals a distinct path of cross-cultural management research (Adler 1983a, Adler 1983b, Adler, Doktor, and Redding 1986, Boyacigiller and Adler 1991, Boyacigiller, Kleinberg, Phillips, and Sackmann 2004, Earley 2006, Earley and Singh 1995, Jackson and Aycan 2001, Joynt and Warner 1996, Kirkman, Lowe, and Gibson 2006, Leung, Bhagat, Buchan, Erez, and Gibson 2005, Roberts 1970, Smith 2001, Tjosvold and Leung 2003, Triandis 2001, Yeganeh, Su, and Chrysostome 2004, Zhu and Ulijn 2005). Collectively and metaphorically, this body of literature paints the voyage of cross-cultural management research as having gone through the following major phases since its inception: an early childhood during the 1960's; an adolescence during the 1970's; a prolific young adult from the 1980's onwards - with Hofstede (1980) and its influence as the landmark; and a crisis of identity during the late 1990's that ushered in the coming of age for cross-cultural management at the start of the current millennium.

As cross-cultural management research now begins to show visible signs of maturity and sophistication, it must face new challenges. Specifically, recent critical debate on cross-cultural management research presents a collective view of the challenges that lie ahead as the field enters its maturity phase (Boyacigiller, Kleinberg, Phillips, and Sackmann 2004, Bresman, Birkinshaw, and Nobel 1999, Earley 2006, Fontaine 2007, Jackson and Aycan 2001, Jackson and Aycan 2006, Leung, Bhagat, Buchan, Erez, and Gibson 2005, Smith 2001, Tjosvold and Leung 2003, Yeganeh, Su, and Chrysostome 2004, Zhu and Ulijn 2005). The challenges identified in this body of literature relate to an issue that call for a research program that acknowledges deepening and widening of methodological approach to support future theoretical mandate. 
The first methodological challenge calls for a broadening of paradigmatic stance (Adler 1983a, Boyacigiller and Adler 1991, Boyacigiller, Kleinberg, Phillips, and Sackmann 2004, Earley 2006, Jackson and Aycan 2001, Leung, Bhagat, Buchan, Erez, and Gibson 2005, Soderberg and Holden 2002, Tjosvold and Leung 2003, Triandis 2001, Usunier 1998, Yeganeh, Su, and Chrysostome 2004, Zhu and Ulijn 2005). Broadening of paradigmatic perspective necessitates openness to paradigms of inquiry other than the one that has dominated the field so far, namely the positivist/post-positivist paradigm and its de-facto affinity with robust quantitative analysis. This should yield a greater variety of views on what are other possible loci of analysis, beside the popular 'culture as essence' and cultural dimension analysis (Holden 2001, Holden 2002) and move research agenda into areas that have been under-investigated, particularly the actual dynamics that transpire in cross-cultural interfaces and the significance of context in such dynamics (Jackson and Aycan 2006).

For this new direction to proceed, incorporation of other paradigmatic stances than the dominating positivist/post-positivist paradigm is needed. This study, with it explicitly adopted interpretive paradigm, contributes to validate not only the potential but also the practicability of such alternative paradigmatic stance. Chapter 2 and Chapter 6 discuss in great detail how the interpretive/qualitative methodology can contribute to add the much needed new perspective to advance research in cross-cultural management in general and cultural intelligence or CQ in particular. This study stands as one incremental addition to this much needed advance.

The second fundamental methodological challenges relate closely to the issue of paradigmatic stance. Broadening of paradigmatic perspective obviously calls for greater variety of methodological choices. Qualitative inquiry, in particular, has been cited as a much needed approach to augment the dominant quantitative research that has been the mainstream in cross-cultural management so far (Boyacigiller, Kleinberg, Phillips, and Sackmann 2004, Earley 2006, Jackson and Aycan 2001, Leung, Bhagat, Buchan, Erez, and Gibson 2005, Triandis 2001, Zhu and Ulijn 2005). Specifically, qualitative inquiry offers investigating ability that could unlock those theoretical issues that are difficult, or even impossible, to explore via quantitative instruments, issues such as the significance of context and actual interaction dynamics often identified as the next frontiers in cross-cultural research. More, qualitative study, as illustrated by this study, allows for simultaneous treatment of different levels of units of analysis - an analytical approach very much needed to investigate interactional dynamics but hardly feasible with quantitative methods.

Against these methodological challenges, this study and its findings - both the resulting grounded theory of XCQ and the illustrative thick descriptive cases - could serve as a showcase, albeit a humble one, for the value and potential of qualitative methodology in cultural studies. Dramatically speaking, the main methodological contribution of this study may be in how it could add yet another fresh voice to the ongoing 'better stories v. better constructs' debate (Dyer and Wilkins 1991, Eisenhardt 1991), by proclaiming - through the interwoven theories and narratives presented in 
this manuscript - that 'better stories are better constructs'. Or to repeat what I asserted earlier in Chapter 2, 'the story is the message', in the fashion of McLuhan' famous expression.

\section{Managerial implications}

The very image of intricate cultural webs accentuates the key message of this study vis-à-vis managerial implications - XCQ denotes the single strategic capability that permeates all transactions in an organization, from an individual level to all higher levels of group interaction. Culture no longer involves the mere force of national culture, as has been so misleadingly over-popularized. Intricate cultural webs entail the recognition that cultural force develops from diverse forms of 'culture' combined national, organizational, biological, social, and all others. Again, this complex situation can only be tackled via repeated practice of simple cross-cultural heuristic. This study's findings suggest that organizations will do well to develop a policy that foster such practice and monitor its impact on organizational process and success. This translates into a framework of managerial implications of XCQ in an organization as depicted in Diagram 8.1.

In essence, Diagram 8.1 simply depicts what already happens on a daily basis in all organizations, even though many may not be conscious of such a process. As this study shows, all cultural units in an organization - individuals, groups or the organizations themselves - must exercise their (perhaps subconsciously developed) cross-cultural heuristic on a daily basis, because such is the demand of organizational life. Aware of it or not, all managers and their employers are compelled to go through the process of 'making sense' of what transpires under intricate cultural forces. The actors in the three cases and following two vignettes in this study all related how they must develop a sense-making tactic in order to wade their ways through the complexity of crosscultural interaction. They may not call this cross-cultural heuristic, but as the stories portrayed, they were actually exercising what this study theorized as a simple heuristic procedure that is at the heart of managerial implications of this study's findings.

In effect, this study makes the subconscious process explicit and puts it at the forefront of how organizations can best manage their own intricate cultural webs. To repeat, at the heart of managerial implications of XCQ is the actual application of this strategic capability - the practice of cross-cultural heuristic expounded in great detail in Chapter 6. In essence, cross-cultural heuristic is XCQ in action. Grounded theory of cross-cultural intelligence as theorized in this study brings to light the fact that every organization is a complex set of cultural units and thus cross-cultural heuristic must obviously be practiced at all levels in an organization - individual, group, organization, and inter-organization. These levels represent both the 'unit' and the 'context' of XCQ in an organization. As a result, any organization that wishes to improve its overall ' $X C Q$ ' must invest in an effort to cultivate, advance, and assess XCQ of all these units and in all these contexts. 


\section{managerial policy domain}

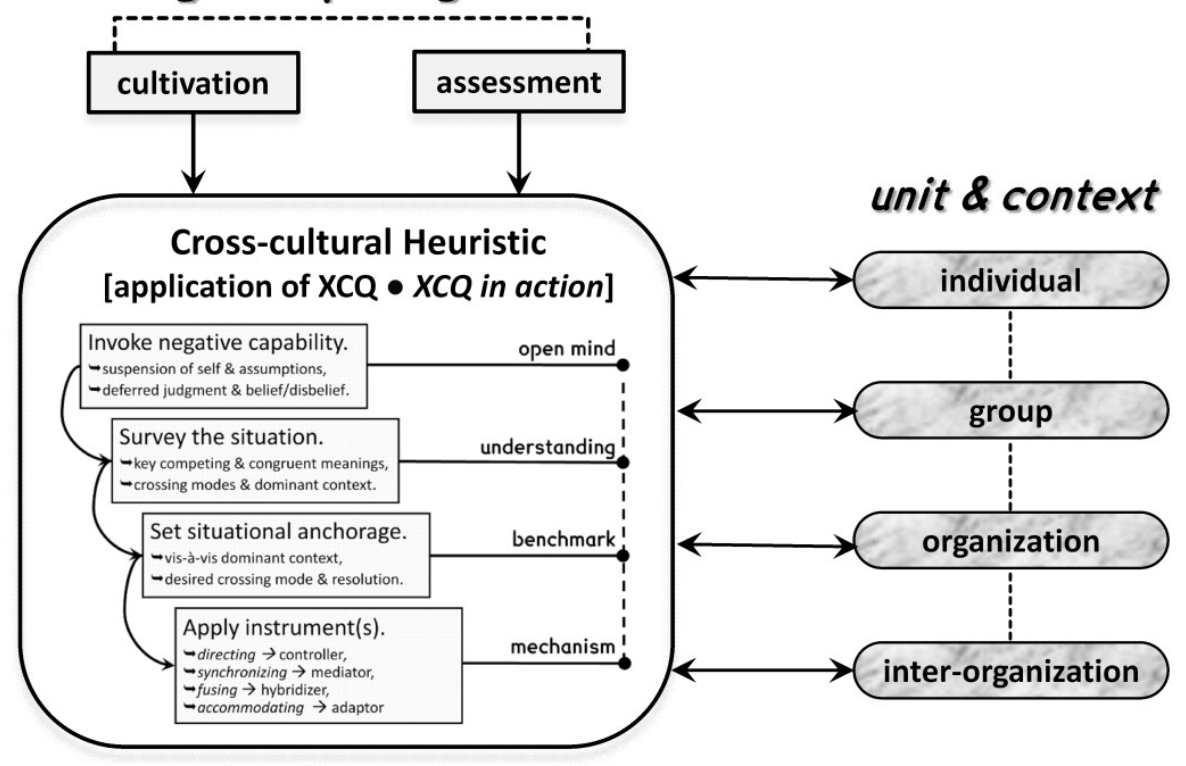

Note: Author's conceptualization based on study's findings.

This very explicit recognition of the implicit and pervading significance of crosscultural heuristic in organizations calls for a program of XCQ development that goes beyond focussing on training of individual expatriates, as has been the most prevailing managerial practice in dealing with cross-cultural situation. In fact, because everybody in an organization, not only expatriates, is a complex cultural unit, organizations will do well to provide support for everybody to constantly improve their XCQ in everyday actual practice as well as through formal learning platform. Naturally, individual employees will vary in their need of such support, according to their particularly cultural profile in conjunction with their work context. A well-thought out policy mandate on XCQ development and application in organization must take all these considerations into account.

More, beyond individual learning, group learning, that is, learning as a group to practice and improve 'group $X C Q^{\prime}$ ', must be an imperative additional policy mandate. Of course, group here denotes a group that is a cultural unit, hence, this policy domain must encompass all significant such groups in an organization - team, department, organization, and even inter-organization groups, in the myriad contexts the group may function in, formal, informal and otherwise. Of particular concern are those incidental or irregular groups such as special project teams that must be formed out of myriad webs of cultures (national, departmental, functional, and so forth) - a very common occurrence in most multinationals. Such teams are highly situational and 
cannot rely on institutional apparatus of formal learning platform. For such teams to succeed, its members - all individual cultural units - and the team itself must be equipped with the capability of learning by doing that is the very character of crosscultural heuristic and XCQ.

Admittedly, this view of managerial implications of cross-cultural interaction and its stipulated policy domains and mandates are far more elaborate than how most organizations currently manage their need to deal with cross-cultural context. Yet, if $\mathrm{XCQ}$ is recognized as an indispensible strategic capability for organizations and their employees, as suggested by the findings in this study, this view proffers the most attractive new path for organization to adopt. Given that, future research on XCQ must address this need to aid organizations in their implementation of the cultivation, application and assessment of organizational XCQ under the framework suggested by findings from this study - the very direction of the road ahead.

\subsection{The road ahead: future research}

The tenet of XCQ in action views XCQ as a strategic capability and calls for application and research to focus on two closely linked issues of cultivation and assessment - how best XCQ can be cultivated and what constitutes the most effective manner to assess the level of XCQ that a cultural unit has developed. The imperative principle of XCQ in action lends significant direction to both these domains.

Two aspects of future research to extend this study are the major research themes to be pursued and the actual research programs that can translate those themes into actual investigations.

\section{Research themes}

Two main research themes that are in pressing need of deeper and broader investigations involve first the issue of cultivation and second that of assessment. Together, these two research theme suggests a wide variety of programs for future research.

\section{Cultivation - cross-cultural practicing and coaching}

Cross-cultural training research and practice have served for many decades as a long standing and prolific effort to help individuals develop the needed understanding and skill to live and work successfully in a cross-cultural environment (Bhawuk and Brislin 2000, Black and Mendenhall 1990). What started as a simple program to prepare expatriate soldiers, missionaries or businessmen before they sojourn to a new country or culture has over half a century advanced in scale, scope and sophistication. Recent advances in the field of cross-cultural research and practice interestingly concur that its direction should now move towards a practiced-based orientation (Bhawuk and Brislin 2000, Black and Mendenhall 1990, Boyle, Nackerud, and Kilpatrick 1999, Earley 1987, Klinge, Rohmann, and Piontkowski 2009, Swift and Denton 2003, Yamazaki and Kayes 2004) - a direction that resonates with the imperative principle of XCQ in action suggested by this study. 
Given that premise, research into the cultivation of XCQ must take on a turn that goes beyond cognitive, psychological, or behavioural alone, but seek to fuse these together via action. Hence, cultivation of XCQ should take a similar approach as that commonly used in development of other tacit skills - an approach that couples practicing with coaching. Conventional classroom approach has proved to be only supplementary and never sufficient for tacit skill development (Davis, Sumara, and LuceKapler 2000, Grotzer and Perkins 2000, Merriam, Caffarella, and Baumgartner 2007, Swift and Denton 2003, Yamazaki and Kayes 2004). Therefore, instead of looking into XCQ cultivation programs that follow conventional classroom orientation, future research should seek to develop programs that put cultural units at the heart of a real, or realistically simulated, setting and let them act and react to the situation and learn from those actual experiences, with trainers acting as coaches, not instructors.

Such research agenda suggests many attractive domains for future investigation. First, better insight into the nature and working of XCQ will help improve our understanding to serve as groundwork for cultivation program development. This calls for further probing into XCQ and its core heuristic procedure as practiced in the real world, under varying contexts. Next, XCQ cultivation programs can be researched and developed along two major platforms: real-world and simulated settings, both of which require further creation and improvement of learning and coaching contents as well as instruments. Real world setting cultivation refers to programs that foster cultural units to learn from their own cross-cultural experiences in real-world settings, be that on-the-job or in daily life. For such real world XCQ cultivation programs, the aim will go beyond letting cultural units strive on their own but include instruments and coaching elements that are framed within the cross-cultural heuristic procedure and reinforce the recursive-reflexive property of actual practice. Simulated setting cultivation refers to training programs that use simulated, yet naturalistic, settings that are well-designed to stimulate cultural units to exercise their XCQ and learn from the 'virtual' experience, almost as if they were in real life. Such programs can employ a variety of well-known instruments such as case, simulation, game, and role-playing, all of which offer possibilities to provide virtual environment needed to induce response in the form of actual action that go beyond cognitive response alone, as is characteristic of conventional classroom type training (Boyle, Nackerud, and Kilpatrick 1999, Klinge, Rohmann, and Piontkowski 2009, Merta, Stringham, and Ponterotto 1988). Again, how to effectively develop and implement such virtual exercise instruments promises to be an appealing research theme, both theoretically and practically.

For both research into the nature and working of XCQ and the cultivation of it, advances in education and learning theories provide useful conceptual foundation. Specifically, scholars in the field of cross-cultural training have already identified two emerging learning paradigms that are compatible with the premise of XCQ in action, namely the experiential learning and situated learning perspectives (Ou 2009, Yamazaki and Kayes 2004). Although competing views of what experiential learning means are still being negotiated, its major tenet that puts experience (real or simulated) as 
the engine of learning and the imperative to adapt and evolve as its objective is well established (Davis, Sumara, and Luce-Kapler 2000, Henry 1989, Illeris 2007, Kolb, Boyatzis, and Mainemelis 2000, Larsen 2004, Mainemelis, Boyatzis, and Kolb 2002, Merriam, Caffarella, and Baumgartner 2007, Quay 2003). This tenet accords fully with the premise of XCQ in action. Situated learning then lends additional attention to the social, contextual and interactive aspects of learning that also corresponds well with the context-oriented nature of XCQ heuristic (Davis, Sumara, and Luce-Kapler 2000, Handley, Clark, Fincham, and Sturdy 2007, Lave 1993, Lave and Wenger 1991, Merriam, Caffarella, and Baumgartner 2007, Ou 2009, Quay 2003, Wenger 1998, Wenger and Snyder 2000). As such, the two learning theory strands offer a rich foundation for future investigation into $X C Q$, its nature and cultivation.

As such, a new research theme that injects a particular attention on cross-cultural heuristic as a major vehicle to anchor experiential and situated learning approaches to cultivate XCQ is in order. Cross-cultural heuristic could well provide an explicit guideline for both the coaches and the learners to reflect on their cross-cultural experience and develop better understanding of both its development and consequence. Specifically, the second and third steps in cross-cultural heuristic procedure (surveying the situation and setting situational anchorage) afford a direct link to situated learning principles, while the four simple steps give a practical and manageable framework for both the learners and their coaches to observe and reflect on the learning-by-doing experience.

\section{Assessment - cross-cultural road test}

Assessment of cultural intelligence, or $\mathrm{CQ}$, particularly in rigorous quantitative manner, has been the main motivating force and central effort in the research on cultural intelligence (Ang and Van Dyne 2008, Thomas, Elron, Stahl, Ekelund, Ravlin, Cerdin, Poelmans, Brislin, Pekerti, Aycan, Maznevski, Au, and Lazarova 2008, Thomas, Stahl, Ravlin, Poelmans, Pekerti, Maznevski, Lazarova, Elron, Ekelund, Cerdin, Brislin, Aycan, and Au 2008) - hence the inherited abbreviated term CQ, which heavily resonates with the popular notion of IQ. Immensely prolific and useful as this stream of research is, it also inherits the limitations commonly associated with the inspiring model of an IQ test, the most important of which are first a detachment from context and action and second the inevitable bias inherent in the self-reporting approach extensively used in such tests. As Gelfand, Imai, and Fehr (2008) pointed out in their deliberation on the road ahead for CQ research, the need to pry open the 'CQ black box' and go beyond restricted quantitative assessment of $\mathrm{CQ}$ epitomizes the frontier of CQ research. Again, I see action as a promising key that could unlock the mysterious $C Q$ black box.

Specifically, injecting the imperative of XCQ in action into the assessment of CQ stipulates that the situational and interactional aspect be a key ingredient of the evaluation process. It also instigates observational and reflexive assessment perspective, in place of the limited self-reporting appraisal. This implies an assessment approach that is completely different and hence highly complementary to the usual CQ 
test. This approach involves an assessment scheme with two interlinked activities. First, the scheme calls for a third party who will observe cultural units and their action in a variety of cross-cultural contexts - again either real-world or naturalistically simulated - and make assessment of the quality of the XCQ of those cultural units, as manifested through their action and interaction in response to realistic situations. Secondly, the scheme must also encourage cultural units to exercise reflexivity to deliberate on their own experience as a learning-by-doing mechanism. The third party observation and self reflexivity could of course be combined to complement each other, much like what is usually done in a usual coaching program.

Metaphorically speaking, this assessment approach will take cultural units on a cross-cultural road test, not unlike a literal road test whereby a coach-cum-examiner must assess the quality of drivers and their actual driving in real-life traffic and the drivers must reflect on their own action in an actual traffic. Pioneering research into the development and implementation of such cross-cultural road test - its specific contents and procedures - will be as challenging as it is rewarding. This can be done naturally in conjunction with research into the development of XCQ cultivation programs. Together, such parallel efforts will initiate an entirely new research theme that aims at a mission to create a totally novel group of cross-cultural professionals - the cross-cultural coaches, examiners, and even 'profilers' who will take charge of training and assessing cultural units in their daily endeavour to wade their ways successfully through the intricate cultural webs.

\section{Research programs}

The above brief survey of the two major research themes suggests ample opportunities for future research programs. Here, I will outline the four research programs I consider to be not only lucrative but also imperative if we were to advance further our research in cross-cultural management in general and cross-cultural intelligence in particular.

\section{Substantiation and extension of current model of XCQ}

One apparent limitation of this study is the limited scale and scope of the substantive platform upon which the proposed grounded theory was built. Obviously, this necessitates a need to substantiate and extend the value and validity of this study's findings further. For that, a program of research that injects richer triangulation elements into the investigation is needed, which can be done by taking the proposed grounded theory and its import into other platforms of cross-cultural context. This will involve two inter-linked investigative courses: horizontal and vertical.

The horizontal extension refers to investigation that examines cross-cultural contexts comprising diverse sets of national cultures, particularly those that vary from the one in this study. A variety of horizontal contexts can be pursued: a replicated setting (e.g. German, American or other Western firms operating in Thailand or other Asian countries); a reverse setting (e.g. Thai, Japanese, Chinese or other Asian firms operat- 
ing in Western countries); or an entirely different combination (e.g. Latin American firms operating in African countries, or vice versa, and so on). The vertical extension then refers to investigation that investigates a diverse variety of cultural units - individual and group, both formal (including project team, as well as departmental, organizational and inter-organizational group) and incidental (such as communities of practice, clique, or even family). Notably, these two expansions of research dimension resemble suggestions on future research made by many prominent scholars in recent years (Boyacigiller and Adler 1991, Boyacigiller, Kleinberg, Phillips, and Sackmann 2004, Earley 2006, Jackson and Aycan 2001, Leung, Bhagat, Buchan, Erez, and Gibson 2005, Peterson 2006, Shenkar 2004, Soderberg and Holden 2002, Tjosvold and Leung 2003, Triandis 2001, Yeganeh and Su 2006, Yeganeh, Su, and Chrysostome 2004, Zhu and Ulijn 2005).

Obviously, the more sophisticated research program as outlined above guarantees as well as entails richer sets of informants and inquirers, which would in turn yields richer information to advance the understanding of XCQ further.

\section{Cultural coaching}

As deliberated earlier, the two related fields of experiential learning and situated learning offers a lucrative prospect for interdisciplinary research program on XCQ cultivation. Given the tenet of XCQ in action and the nature of experiential learning and situated learning, there is a pressing need to augment the limitation of quantitative approach to investigate XCQ cultivation effort (Bhawuk and Brislin 2000, Brislin, Worthley, and Macnab 2006, Gelfand, Imai, and Fehr 2008, Grotzer and Perkins 2000, Jackson and Aycan 2006, Soderberg and Holden 2002, Sternberg 1997, Yamazaki and Kayes 2004, Yeganeh, Su, and Chrysostome 2004, Zhu and Ulijn 2005). Evidently, a qualitative approach to design and develop learning environment that promotes crosscultural intelligence of a cultural unit that is based on live observation and coaching of $X C Q$ in action - taking the learners out of the lecture and onto the road - offers great opportunities for future research. This investigation into XCQ cultivation will thus focus on two tracks of XCQ learning and coaching. First will be a study into the development of procedures and mechanisms that enable learners and coaches to reflex on actual everyday cross-cultural practice in an organizational setting. Second will be development and testing of simulated instruments and environments, such as cases, games and role-playing exercises that can be used to effectively facilitate both learners and coaches to engage in virtual cross-cultural practice as a way to enhance XCQ. These two research tracks must of course include consideration of individual as well as group learning, and can benefit from linkages with existing research effort on leadership in cross-cultural context as well as that on intercultural team (Gelfand, Erez, and Aycan 2007, Hofstede 1980, House, Hanges, Javidan, Dorfman, and Gupta 2004, Janssens and Brett 2006, Schein 2004, Soderberg and Holden 2002, Tjosvold and Leung 2003). 


\section{$X C Q$ road test and cultural profiling}

In parallel to research on XCQ cultivation, research into XCQ assessment based on the principle of XCQ in action is needed to complement the now flourishing work on quantitative CQ measurement, as earlier deliberated. To my knowledge, development of a comprehensive valid XCQ 'road test' is virtually non-existent. Naturally, individual cultural units can take a few respectable CQ test to gauge their CQ (Ang, Van Dyne, Koh, Ng, Templer, Tay, and Chandrasekar 2007, Ang, Van Dyne, Ng, and Koh 2004, Thomas, Elron, Stahl, Ekelund, Ravlin, Cerdin, Poelmans, Brislin, Pekerti, Aycan, Maznevski, Au, and Lazarova 2008, Thomas, Stahl, Ravlin, Poelmans, Pekerti, Maznevski, Lazarova, Elron, Ekelund, Cerdin, Brislin, Aycan, and Au 2008). Yet, as already discussed, CQ is only an indicator of the 'potential', not the 'actual' success of an individual to operate in a cross-cultural context. At a group level, moreover, such CQ measurement is nonexistent, understandably because of the complexity inherent in designing robust quantitative measurement instruments for such complex units. Qualitative approach to XCQ assessment, by contrast, offers a feasible alternative to assess cultural units at both individual and group levels. An elementary inspiration regarding this research track is to explore the possibility of applying the concept of 'psychological profiling' to the assessment of XCQ. Although the term 'cultural profiling' may or may not prove to be viable, it still provides an intuitive inspiration on how to approach study on assessment of XCQ - an inspiration that awaits further exploration.

\section{Advancing qualitative research}

Lastly, as a spill over from the above qualitative focus on XCQ investigation, future research effort under the above three research programs should undoubtedly contribute to the advancement in qualitative research. Specifically, more rigorous use of many other interpretive/qualitative procedures and instruments, particularly participant observation, ethnography, auto-ethnography, and focus group, as part of methodological triangulation will strengthen and advance both the quality and status of qualitative approach in cross-cultural research (Dyer and Wilkins 1991, Eisenhardt 1991, Gephart 2004, Jackson and Aycan 2006, Osland and Bird 2000, Ulijn 2000, Wright 2004). More, a reinforcement of methodological triangulation through the prospect of combining these diverse qualitative research methodological genres as well as combining them with quantitative methods also promises lucrative opportunities to accentuate the role of qualitative approach in the emerging mixed-methodology paradigm (Creswell 2003, Tashakkori and Teddlie 2003, Teddlie and Tashakkori 2003, Tobin and Begley 2004). With this intention, future research endeavours in any of the above three programs, if valid and significant, will contribute to future advancement in qualitative research - its rigor as well as status among research communities in organization and management in particular and in social sciences in general. 


\subsection{Back to the future: limitations}

Most scholars often exploit the option to exercise 'an unequalled gift, especially pen in hand, of squeezing big mistakes into small opportunities ${ }^{35}$, although under a less dramatic tradition of the customary section on limitations in the concluding part of research reports. I will now attempt to also squeeze a few big and not so big mistakes into small opportunities in the same fashion.

\section{Origin and illustration}

Like most, if not all, research, this study was constrained by a few limitations, arisen from various sources. Most of these have already been addressed throughout the manuscript, mainly by means of reflexive notes. Hence, in this concluding deliberation, I will concentrate only on those limitations that serve as big 'disclaimers' to readers and small learning opportunities for scholars, myself obviously included. These limitations have to do with two important aspects of the study: its form and strategy.

First are those limitations inherent in the very form of this study - namely a doctoral dissertation research. As a doctoral study project, this investigation must suffer from being a kind of 'one-man' (or indeed 'one-woman') show, albeit one guided by an established director and producer. Added to this is the drawback of having the very one woman of the show being inevitably a novice, who must confront the challenge of learning by doing head-on and hope to learn from her 'educated' trial and error. Last is a constraint common to all research but arguably more acutely in a doctoral study the mundane economic principle of scarce resource and opportunity cost which demands us to prioritize what could be done given limited resources, particularly time and money, both of which were certainly not available in abundance for a doctoral research project such as this.

Next, the strategic choices made for this study also introduced certain challenges, despite, or actually because of, the benefits they offered. Methodologically, the interpretive grounded theory approach, as deliberated in Chapter 2, calls for acute awareness of the burden of interpretation, both on the researchers and their audience. That is, study results must be carefully taken as an 'informed' interpretation made by a particular interpreter with a specific profile. Also, because grounded theory is a relatively young methodology, the opportunity to learn from previous experience is somewhat limited. Then, theoretically, the theme of cultural intelligence, as repeatedly mentioned, is an emerging research theme and most studies on this topic will turn out to be exploratory in nature at this early stage. To exploit my early metaphor, I chose the road less travelled to serve exploratory purpose, and must address with extreme care the difficulties and limitations that came with that deliberate choice.

\footnotetext{
${ }^{35}$ Here I refer to the famous quotation, 'She had an unequalled gift, especially pen in hand, of squeezing big mistakes into small opportunities.' - in Henry James' Graville Fane, 1881.
} 
To relate these limitations and how I dealt with them, I will use a stark indication of the import they have on this investigation - two vignettes that are the remains of two incomplete cases I failed to develop into full thick descriptive cases as originally intended. The vignettes, of some use as they may have been as part of field evidence, were even more useful as big mistakes upon which important lessons and opportunities could be harvested. To recall, the first interviewing phrase in the Netherlands yielded five companies that promised great potential to provide grounded evidence for five rich thick description cases. I thus set out to investigate all five during the field work in Thailand. Two of the five candidates failed to materialize for different reasons. These two 'failed' cases provided valuable research experience and afforded a number of worthy investigative lessons.

\section{Vignette TMF - an accidental pilot case study}

TMF was the company that set off this investigation. Khun Herman de Bruin, the company's top executive, was among the first few Dutch respondents who accepted with enthusiasm my invitation to participate in this study. He then became the very first informant to be interviewed. Khun Herman and his story of the Thai subsidiary of a medium size Dutch multinational eventually turned out to be an unintentional pilot case study in more ways than one.

\section{TMF: getting to know you}

TMF was a medium, fast growing Dutch manufacturer and supplier of construction products for building insulation purposes, such as special pipes and insulation materials. All its activities, from production, distribution and sales \& marketing were done in house, using virtually no outsourcing. Its clients were originally construction businesses, although as the company grew, it had also recently branched out to consumer market of home-owner and doit-yourself segment.

The company started off in 1976 as a small manufacturer in the Limburg area of the Netherlands. It had since continuously expanded its operation, first to neighbouring countries of Belgium, Luxembourg, and Germany and gradually further away both within the European continent and more recently to other parts of the world. By 2007, its expansion in Europe had spanned as far as Turkey, Poland, and Russia and its international reach covered such regional hubs as Mexico and Thailand. Due to the nature of its products, which required local adaptation and high transportation cost for finished merchandise, TMF needed to establish a local base for all its core activities in any region it expanded to, starting with a sales \& marketing office, followed by a logistical distribution centre, and completing with a local production site, once its business had gained enough volume. This expansion strategy had served TMF well, keeping transportation cost of bulky finished products low when shipped from local production sites, while 
maintaining harmonious management practices for its overall operation. In general, TMF carried out its expansion in this typical and deliberate fashion, though not entirely so with its entry into the Thai and Asian market.

\section{The view from the top - Khun Herman}

Dhr. Herman de Bruin, TMF's core executive who had been with the company from day one, was in charge of expansion activities. Regarding Thailand, he stated bluntly, 'We went into Thailand for all the wrong reasons'. He recalled how one individual, a German working for TMF's German partnering firm, made it obligatory for TMF to agree with an expansion into Thailand as part of a larger collaboration deal. The agreement was that the expansion must be handled by that German personnel, who claimed to have experience with Thailand. It sounded like a good idea at that time: it was in the early 1980's when Asia's economic rising and its accompanying construction boom painted an attractive growth opportunity for a growing company in search of new ground. The prospect was lucrative, but the execution plagued with problems for TMF, primarily because of the German partner's private agenda - for him to be in Thailand to pursue a personal relationship with a Thai lady. For the first six years, when this German partner was in charge of TMF's Thai expedition, nothing went right, because the man in charge let his personal agenda dictate business decisions.

Thus entered Khun Herman, to take charge and get it right for TMF Thailand. The German partner was dismissed and Khun Herman started building TMF Thailand virtually from scratch, recruiting new key personnel and setting out to train and mould this new team to manage the Thai operation, which was to eventually be TMF regional headquarters for the entire AsiaPacific operation. The long-term strategy was to have a team of three key managers, one for each of the major activities - finance \& administration, factory \& production, and sales \& marketing. Once this team worked well together, one of the three would be appointed General Manager and put in charge of the Thai and Asian operation, with Khun Herman still its Managing Director but supervising only in a hand-off manner. This objective had proved to be not that easy to realize, although after fifteen years or so, Khun Herman believed he was near achieving it.

After a few trials and errors in recruiting key managers, Khun Herman believed he had now got the 'right' team of key top managers who were competent in their own areas. More, he had managed to blend the needed Dutch business philosophy with the Thai culture in the interrelationship of this 'team of three' such that the factory \& production manager, Khun Prasert Laksakul, could soon take the lead of the Thai business as General Manager. Khun Prasert was senior enough to demand respect from the other two younger key managers. After a few disappointments with highflier, foreign-educated engineers who turned out to be concerned only with 
their own career and too thin-skinned for the down-to-earth business of construction materials, Khun Herman was surprised how a self-made, locally educated technician that Khun Prasert was could be very compatible with TMF's business mandate and corporate culture. Khun Herman saw great potential in Khun Prasert as the sought-after 'local' head of TMF.

Thus, in 2006 after over a decade of hand-on supervision of the Thai operation, Khun Herman hoped to soon distant himself from the day to day matters of TMF Thailand and concentrate more on moulding his new found prodigy - Khun Ekachai Samard, the new Export Manager. Market development was Khun Herman's original area of expertise and he saw much potential in Khun Ekachai to help build a lucrative market in the entire Asia Pacific region. As a Thai Muslim who was unexceptionally tall, looked more like a South Asian than a Thai, and spoke fluent English with little trace of the usual Thai accent, Khun Ekachai had the right ingredients to break through the common stereotyping of Thais as inferior business counterparts - a stereotype held by many of TMF's clients in the region, particularly in Singapore, India and Australia where TMF was actively building a market. Khun Herman expected great future for his new found prodigy who possessed both the look and the talent to operate at the international playing field. Khun Herman must admit he was rather fond of this young man.

\section{The view of a rising star - Khun Ekachai}

Khun Ekachai, however, would probably be surprised if he were to be told what Khun Herman thought of him. Khun Ekachai respected his Dutch boss very much and appreciated highly the opportunity given to him by this firm. Despite his 'non-Thai' appearance, he was born and raised as a Thai and his look would not deceive any of his Thai fellows at all. His short period abroad as a student may have helped him understand the Western way enough to work the international business scene, but that did not make him a Westerner. After having worked closely with Khun Herman, visiting various clients throughout the region and working out marketing plan with the top boss, Khun Ekachai came to view the Dutch MD less as a foreign boss and more as a paternal mentor.

That was why he could not help feeling hurt and upset when he went to pick up Khun Herman at the airport last time, just like every time Khun Herman visited Thailand. This time was different to Khun Ekachai, though. It was only just after his wife was admitted to a hospital due to a serious illness. For a young family like his, this was a dramatic event and he was still shaken up by it. Khun Herman knew about this, surely, because he had been informed of Khun Ekachai's emergency absence from work while the MD was away from the Thai office. Yet, the first thing Khun Herman talked about, after the usual short greeting and settling down in the car for a long ride to the office, was to inquire after the various marketing projects they were 
working on. And they were not even in the office yet. The ride from the airport was supposed to be a casual time, just the right setting for Khun Herman to inquire after personal affairs. Instead, the boss jumped right into business as if nothing had happened to upset Khun Ekachai. Hurt and disconcerted, Khun Ekachai simply did not understand how Khun Herman could be so unconcerned. Surely, he liked me, Khun Ekachai kept telling himself, but how could he ignore my tragedy just like that, when even the colleagues I hardly had anything to do with all conveyed their sympathy to me? And he was my mentor, someone I worked with most closely in this firm. How could he not be concerned about something that was so important to me? My young wife was hospitalized, for goodness' sake.

Still, they kept at their work and Khun Herman seemed as happy as ever about the work prospect. It confused Khun Ekachai even further.

\section{View from the emerging top}

Khun Prasert, the factory \& production manager, observed the Bangkok office with interest every time he visited it. His 'home-base' was at the company's factory in the industrial area outside of Bangkok, and he visited the Bangkok office only occasionally, mostly when Khun Herman visited Thailand and wanted to have a general meeting with all key managers. The Bangkok office was more of sales and marketing base and most of TMF's ground operation took place at the factory. In a way, everybody operated more as a satellite here at TMF, each doing his own thing. Khun Prasert and his factory, for example, were the working bee satellite for TMF, churning out products for the other parts of the firm to sell them.

Khun Herman had given him a hint that soon Khun Prasert would take over the helm of TMF Thailand, when the Dutch top boss would lessen his grip on the Thai day-to-day operation. Khun Prasert thought he was ready for that. He had a good hold on the factory, after exercising enough of both hard and soft power struggle with the 'cowboy' temperament of the factory workers. It helped that Khun Prasert came from a tough background, with his history as an amateur Thai kick boxer and his familiarity with the rough disposition of Thai polytechnic education. His skin was thick enough to withstand the resistance of the workers at first, whereas his self-financed higher education helped him earn their respect. After a few years, he had the factory under control.

The question was, how he would get the whole TMF operation under control when the time came. The other two key managers held enough respect for him, of course, since he was far more senior and had been with the firm the longest. But each of them had so far functioned independently, with Khun Herman acting as a sort of occasional tie. Look at even within the same department of sales \& marketing, the two Thai sales personnel, who hardly spoke English and only worked on the Thai market, carried out their 
sales completely differently from the way Ekachai and Khun Herman worked with their international clients. But Khun Herman seemed to be fine with that, as long as they generated sales, of course. It seemed to be this way with TMF - you did your own thing and as long as the results showed, the Dutch boss would be happy enough.

Would it stay that way?

Accidentally, TMF turned out to be a de facto pilot study in both technical and analytical aspects. The very first formal interview with Khun Herman provided several conceptual as well as practical tips, tricks and ideas for future interviewing efforts including what questions to ask and how to phrase and time them, the need to suppress interviewer's urge to make too many comments (aka knowing and talking too much), the importance of non-verbal clues, and various other practical considerations. Methodologically, the most important and costly 'pilot study' lesson that TMF provided was that the simplest mistake could make or break a case. In this instance, TMF could have become a rich, thick-descriptive narrative and provided prolific informative grounded evidence if not for the malfunction of the recording device during the interviews with TMF's two Thai informants, Khun Prasert and Khun Ekachai. The cassette tapes that were supposed to have recorded the two interviews - each lasting about two hours and covering several incidents and observations - somehow did not record anything, presumably because it was not operated properly. Thus for TMF, only a full transcript of the interview with Khun Herman and some field notes of the two interviews with Khun Prasert and Khun Ekachai comprised the entire grounded evidence. Although this contained a great amount of information that proved to be useful for comparative analysis purpose, I had to conclude that it was not enough for a legitimate thick descriptive narrative - admittedly a rather costly technical mistake.

Yet, the evidence that could be salvaged from the technical blunder turned out to be surprisingly useful during the analytical process. Most significantly, the crosscultural interaction that transpired at TMF illuminates possible 'divisional' resolution state of a 'clashing' cross-cultural condition, as analyzed earlier in this manuscript. More, the fact that TMF operated relatively successfully under this 'clashing' mode and appeared to have developed a particular divisional state that served its operation efficiently suggested that a cross-cultural mode cannot be judged effective in and of itself. Rather, it is the compatibility between the context and the resolution that indicates the level of cross-cultural intelligence exercised in that particular incident. As such, even a clashing situation - revealed through how each party at TMF operated independently - could be a productive resolution state, given a particular context. Although TMF could not be further analyzed in greater details, the vignette hinted that the divisional state at TMF was partly a result of an exercise of cross-cultural intelligence to a certain degree.

TMF was thus a case of 'getting to know you' at two levels. Methodologically, experience from the case helped increase my familiarity with the actual interviewing process, its mechanics and tactics - a pilot study that fostered a learning-by-doing 
investigating skill. Analytically, TMF was a case that illustrated the incident in which two cultures were in the process of attempting to know each other and operate 'together apart' to serve a mutual purpose despite discernible divisive force, or perhaps actually because of it. The relationship between Khun Herman and Khun Ekachai illustrated this process of 'getting to know you' at individual level, whereas the functioning of TMF Thailand, as observed through the eyes of Khun Prasert, highlighted the same process at a group level.

Perhaps I could argue that TMF illustrates one instant where a costly mistake was fortunately squeezed into a valuable opportunity, even during the course of the study.

\section{Vignette ITV - a stillborn case}

Whereas TMF became a failed case turned pilot study due to technical blunder, ITV, a highly promising case, ended up a stillborn prospect, undeliverable due to acute procedural complication. As a stillborn case, ITV highlights the hard reality of field study that often challenges the limit of investigator's ability and research design's ideality.

\section{ITV: behind closed doors}

ITV - a large multinational and world leader in animal healthcare industry had a long and colourful history. Through a series of high-profile merger \& acquisition deals, ITV had been part of a few global corporate groups, all major players in knowledge intensive chemical industry. At the end of 2007, ITV became a new addition to a large US based multinational, after having operated as a subsidiary of another giant European based multinational for many decades.

ITV's origin dated back to the late 1940s when it began as a Dutch laboratory that developed and marketed poultry vaccines. By 2007, ITV had grown to generate global annual revenues of over three billion Euros and hence ranked among the top three of the world's leading animal health companies and Europe's top performer in animal health sector. ITV had achieved its status through a combination of organic expansion and strategic acquisition designed to strengthen its position in key markets and product segments.

ITV's catered to market concerning animals in both food-producing and companion animal segments - from farm animals to domestic pets. Its product portfolio embraced the full range of animal healthcare needs, from prevention to treatment and control of disease, for the worldwide veterinary market. The business was supported by comprehensive research and manufacturing capabilities, coupled by a global distribution network that served more than 140 markets. ITV operated subsidiaries in over 50 countries around the globe and employed a total workforce of well over 5,000. ITV headquarters situated in the Netherlands, in a small town where its first laboratory originated. 
ITV's operation abroad involved three major activities: market development (sales and distribution), research and development (R\&D) and production. Market development served as the base activity in all its offices, whereas R\&D and production were strategically established in only selected countries in each region ITV had a presence. Europe remained the company's dominant site for main R\&D and production while R\&D and production carried out at other regional sites served mainly localization needs. In any case, due to the extreme significance of quality control associated with the nature of its products, all of ITV's products, regardless of where they were produced, must come to its headquarters in the Netherlands first for strict quality control. Once they passed the quality test, they were then shipped from the Netherlands to their destined markets throughout the globe. This process underscored the importance of constant interactions throughout ITV global operation network.

Due to the nature of the industry and its products, ITV's core asset resided in the high-tech value embedded not only in all its products but also in the personnel who developed, delivered and administered them. This translated into an acute reliance on human resource. ITV's key group of personnel thus comprised highly educated and specialised professionals, particularly chemists and veterinarians. Even its sale \& marketing force consisted of only those educated in the advanced chemical and healthcare technologies who had specialized knowledge of the development, production and use of the products. In all its offices worldwide, ITV's hired employees who ranked among the top in those countries' workforce. The same applied to its office in Thailand.

\section{The birth of ITV Thailand}

ITV's initial entry into the Thai market was motivated primarily by market expansion need: to develop the Thai and Asian market for its products. In the early 1970's, ITV formed a distributing partnership with the most prominent local Thai company that was a major player in animal-related markets both in Thailand and the Asia-Pacific region. For the next two decades, the company's activities in Thailand involved only sales and distributions of its animal health products through a tight partnership with this Thai partnering corporation that started off as ITV's first major customer before it became also ITV's customer-cum-distributor. Although this special arrangement worked successfully at the start, ITV's business had grown over two decades to a volume that called for the Dutch multinational to set up a full-blown operation of its own. Also, as business grew, the relationship with the Thai partner as both customer and distributor had become incompatible with the increasing sophistication of the market. ITV considered the market and the industry to be substantial and mature enough for a local office of its own. 
In 1996, Dhr. Rutger Aalbers was thus appointed Managing Director of ITV (Thailand) Ltd., a fully-owned subsidiary of ITV. Prior to this, Khun Rutger worked as ITV executive expatriate in charge of its Indonesian operation. He commenced to build the Thai subsidiary, focusing only on sales \& marketing capability. In 1999, ITV acquired a local company as part of its expansion, thereby quickly enlarged its Thai operation.

As of 2006, ITV Thailand had about 30 employees. The Bangkok office still served mainly marketing and distribution functions. There was no R\&D or production facility in Thailand and the company had no immediate plan for such facility in the foreseeable future. Apart from a handful of administrative staff, the majority of the company's employees in Thailand, all of whom educated in veterinarian science, worked mainly in business development which involves sales, marketing and after sale services. Notably, business development for ITV's products demanded close and continuous relationship with customers as well as other professionals in the field, such as university professors, researchers and veterinarian practitioners. Thus, ITV's staff needed to demonstrate good knowledge of the products and their use in order to establish the firm's credibility. Tight networking with customers and alliances through the business development team was vital to ITV's business. The team carried out a wide range of activities, from organising technical seminars, liaison with the academic, to extensive field work with clients.

After four years, Khun Rutger Aalbers left the Thai office in the hand of a local senior employee, Dr. Sutat Santhornkun - the new Managing Director of ITV Thailand. Ties to the headquarters and global operations were maintained via the leadership and supervision of Dhr. Martijn Lambertus, Regional Manager Asia/Pacific, who managed the network of ITV operations in the region from his base in the Dutch headquarters. This transitional strategy of first sending a lone expatriate to set up a new site and quickly train a local senior executive to eventually head the local office was in line with the general practice of ITV for its operations worldwide. The rationale had to do with the high cost of expatriates and the effectiveness of having local staff handle local operations. The needed link to a local network of veterinarians and professionals proved to be better developed and sustained via local personnel than by expatriates who would always remain 'an outsider' to the close-knitted community of veterinarian practice. Hence, ITV preferred to have local offices headed by trusted and well-trained local managers, such as Dr. Sutat, himself a reputable veterinarian.

\section{Smile that could mean grudge and yes that could mean no}

Khun Rutger and Khun Martijn shared one mutual impression of the Thai people and their Thai colleagues - the famous Thai smiles were as mysteri- 
ous as they were frustratingly ubiquitous and a Thai 'yes' could mean a thousand different things, including 'no'. They both learned soon enough that behind every Thai smile was always a message that must not be associated instantly with humorous disposition and a Thai 'yes' was seldom a simple affirmation. Just as a Thai 'yes' could mean anything, from total agreement, partial hesitation, to suppressed objection, a Thai smile could express a variety of expressions, from sheer joy, covert embarrassment, to subtle vindictiveness. Most Thais seemed to have the instinct to decipher these puzzling yeses and smiles; to Dutch sojourners like Khun Rutgers and Khun Martijn, however, this ambiguity proved utterly exasperating to deal with. Having to decode these yeses and smiles were like trying to see future in a crystal ball or guess what transpired behind closed doors.

Khun Rutgers learned to pry open the closed doors simply by having to deal with them on a daily basis during his four years in Thailand. He started with a practical strategy, of course - he simply recruited those Thais that seemed to be the more inclined to openness. From that, he tried to create an environment where straightforward criticism - something most Thais would have difficulty with - was strongly encouraged and well rewarded. On his side, Khun Rutgers observed how the Thais appeared highly astute in using and reading gestures and non-verbal expressions, instead of words, to deliver messages. His own secretary could often read him like an open book from his non-verbal, often unconscious, body language. And then you had the Thai jokes and how the Thai often used them not at all to entertain but more to convey subtle messages that were too unpleasant to deliver directly. That was something you would never appreciate unless you had to live with it.

So, while Khun Rutgers learned how to decipher, and even use, indirect means to convey messages, his Thai staff learned to take Khun Rutgers' call for openness. In a sense, maybe he could claim to have the closed doors left a little ajar, at least in the ITV Thai office while he ran it. He learned years afterwards that his team was not very happy when he left them. They were anxious about the new general manager - a senior, stern Thai who seemed unlikely to be as open to deal with as their first and departing Dutch boss. Khun Rutgers wondered what to make of that.

Now that Khun Rutgers had moved on elsewhere, it was Khun Martijn who needed to deal with the closed doors of the Thai office. As the Regional Director, though, Khun Martijn could not afford to work so closely with the Thai office. He had several other offices to deal with and his priority was not to have to pry the doors open himself. It's the Thai General Manager, Dr. Sutat, who must be responsible for having the doors open to him, if and when needed. So far, it seemed to work relatively well, at least for the purpose of the business. Headquarters generally had what it needed from the Thai of- 
fice and the people there continued to work and deliver as they should. Still, Khun Martijn couldn't help wondering what they think of him, as their colleagues, and perhaps their friends. It's often a mystery to him most of the time. Unfortunately, it's hardly practical for him to spend time and effort on making the Thai's office doors wide open to him. Not that he was sure that would be for the better.

Yet, it's a nice little mystery to ponder on - what lay behind those closed doors.

The doors of ITV Thailand were agreeably ajar for Khun Rutgers, enticingly open as necessary to Khun Martijn, but mysteriously close to me as a researcher. This fact remained the one single big mistake that I found very hard to digest. Reflexively, the experience provided valuable lessons and raised interesting questions for future investigation efforts. What does it mean when a researcher fails to pursue promising field? What can or should be done in such cases?

During the field work in Thailand, I approached the ITV Thai office in the same manner that I did with the other four prospective companies. Prior to my arrival in Thailand and with reference to the Dutch executive contacts developed in the first interviewing phrase, I sent out introductory e-mails, addressed directly to those individuals referred to me by the initial contacts in each of the candidate companies. These were then followed up by telephone conversations as soon as I arrived in Thailand, which then concluded with exact arrangement for field visits - except in the case of ITV. For ITV, after having received no reply to the first e-mail, a few more e-mails followed. When these e-mails still motivated no response, I decided to make uninvited phone call to the general manager of ITV Thailand, Dr. Sutat, and finally managed (after several failed attempts) to have a short conversation about possible field visits and interviews with some ITV Thai staff. Although Dr. Sutat agreed to try to make arrangement with his personnel - and did send out an e-mail to a group of personnel concerning the matter - I heard nothing further from either Dr. Sutat or anyone else at ITV Thailand. I tried a few more phone calls but could not reach Dr. Sutat. In the last week of the scheduled field visit to Thailand, I gave up and decided to pursue ITV no further. Limited resource simply constrained an extension of my field visit.

On hindsight, the question remains what exactly went wrong in the case of ITV Thailand. Undoubtedly, my inability to open the doors to ITV Thailand played a critical role. I must acknowledge that my enthusiasm about ITV Thailand started to wane after I realized and 'felt' that the Thai office somehow had little interest in participating. I formed this interpretation not from any direct evidence, but, as Khun Rutgers observed, in a characteristic Thai manner - from non-verbal clues that I could detect in numerous indirect signs. As a Thai, I tended to be very sensitive - some might say oversensitive - to this kind of indirect messages. In the end, I decided that it would be neither productive nor ethical to push the case any further. This, despite my regret that ITV promised to be such a rich and different source of evidence. ITV had both common and contrasting characteristics with all other four companies and its experience could 
provide rich information for constant comparison analysis. No doubt, given my subscribed view of researcher as 'research instrument', especially for interpretive study, I was an ineffective instrument in this particular instant - a critical defect that must be addressed and repaired for future use.

The case of ITV thus raised many questions I wish to tackle further in my future career as a researcher. First, I could be wrong in my interpretation, which means the difficulty in arranging field work with ITV might have been a simple matter of the potential informants' busy schedule, or other functional mishaps. If that had been the case, what could have been done to test my interpretation? Would it have been proper to resort to Khun Martijn - whose genuine interest in having the Thai office investigated was quite apparent - and request his intervention? How would such intervention be perceived by the Thai office and would that bear any effect on the field visits and interviews if the investigation were to have taken place? On the other hand, I could be right in my interpretation and ITV Thai staff, for some reasons, simply did not welcome my investigation. Then, this fact itself deserved further probe into the meaning of such reluctance. That, in turn, should motivate even greater research curiosity because it suggested that ITV could offer extremely rich grounded evidence that was different from the cases of willing informants that applied to the other four cases. Still, would unrelenting persistence to pry open the doors to ITV Thailand in the face of such unwillingness be an ethically responsible act? After all, one critical rule of conduct regarding human subjects in any research is that they must fully and openly consent to being part of an investigation.

ITV Thailand, as a failed and stillborn case, raised more questions than it answered. Still, these 'unanswered' questions served to keep the past and future inquiry interesting. They link failed experience to future opportunities and contribute to making the journey a destination.

\section{Implications}

The limitations - arisen out of the constraints of form and strategy, and portrayed above by means of the two failed-cases-turned-vignettes - carry two major implications. First, they illustrate once again that the success of a study should not be measured by the presence of undesired adversities, but rather by how those adversities are dealt with, or even better, turned into opportunities. Second, they confirm the crucial and indispensible role validation methods and procedures play in all research.

\section{The two vignettes - deficiencies turned opportunities}

A legitimate concern regarding the presence of the two vignettes relates to the extent of the effect they have on this entire study - its legitimacy, validity and value. Do the 'remains' of the two prospective cases denote deficiencies or opportunities, to put it bluntly? I would argue that these are two sides of the same coin, which applies also to all research. We do not live in a perfect world and it would be naive to expect that we could perform an inquiry without having to deal with unexpected and undesired ele- 
ments. Even in conventional survey or experimental research, there are always responses that do not meet the rigorous qualifications of statistical analysis and must be dealt with one way or another, either by applying special treatment or discarding them altogether. Yet, these sub-standard responses do not necessarily disqualify research outcome, as long as the entire study meets the normal validity test.

The same principle applies to qualitative study such as this. As discussed at length in Chapter 2, I would argue that this study has met the standard validity test for qualitative research. In the end, having only three cases corresponds well with suggestions made by prominent qualitative scholars that, for studies that deal with thick descriptive case analysis, it is optimal to limit the number of cases to be between three to five, depending on how 'thick' the cases are (Creswell 1998, Creswell 2002, Miles and Huberman 1994, Sandelowski 1995). As it turned out, the three thick descriptive cases proved to provide rich enough evidence to yield valid grounded theory. More, I must admit that if I were to have to analyze and present five, rather than three, thick descriptive cases, it would likely overextend the analytical attention of both myself and the audience, an impediment explicitly cautioned by Mile and Huberman (1994) and Sandelowski (1995).

Eventually, I decided to give the two failed cases a similar treatment other researchers usually apply to their 'not so robust' responses - I squeezed the deficiencies into two small opportunities. Instead of regarding them as mere failed cases, I saw the opportunity to analyze and report two vignettes. Vignette TMF As already discussed, served nicely as a good pilot study and also provided some evidence valuable to the constant comparison analysis for a few concepts, particularly on the taxonomy of cross-cultural condition. Vignette ITV, of a more limited value theoretically, raised a few crucial methodological issues for future debate in qualitative study. Thus, the two vignettes provide constructive, if limited, theoretical as well as methodological value; they are, in effect, two regrettable deficiencies turned gratifying opportunities.

\section{The indispensible value of validation methods and procedures}

Specific to this study and its limitations, all of the validation methods and procedures reviewed in Chapter 2 and summarized in Diagram 2.9 proved to have been most indispensible. Without them, there might have been more and greater limitations. Yet, on looking back, I must acknowledge that if the project were to have been blessed with more resources (investigator, time and finance), the study could have benefited even further from a more robustly employed validation effort. This is a crucial lesson for future research that suggests many implications.

Most lucrative will be a more comprehensive use of triangulation in all possible aspects, particularly triangulation of inquirers (Altheide and Johnson 1994, Cohen and Crabtree 2008, Cohen and Crabtree 2006, Creswell and Miller 2000, Golafshani 2003, Healy and Perry 2000, Lincoln 1995, Long and Johnson 2000, Malterud 2001, Patton 1999, Seale 1999, Silverman 2004, Tobin and Begley 2004). Obviously, having only one researcher in a study is a critical limitation. Had this study been conducted by a team 
of researchers, drawbacks and biases of an individual investigator could have been compensated by intervening and complementary actions and views of others in the team. Triangulation of informants, platforms, and methods, will also enhance research rigor and value - a prospect I have already mentioned in the earlier discussion on future research.

Next, a more rigorous application of prolonged engagement and persistent observation could have definitely minimized the ill-effect of many errors (Cohen and Crabtree 2008, Cohen and Crabtree 2006, Denzin and Lincoln 2005, Healy and Perry 2000, Silverman 2004, Spencer, Richie, Lewis, and Dillon 2003). Admittedly, the field work for this study was relatively limited and short. For thick descriptive cases to be rich, researchers should make every effort to 'embed' themselves as deeply as possible with the actual 'field' and action they are investigating. I am now even of the opinion that a powerful, albeit still controversial, new application of participation observation that embeds researcher as a 'mole' as part of field action (den Hertog and Verkerk 2007) may be a powerful technique to explore in qualitative grounded theory. This deep embedding technique could be the next frontier in the application of prolonged engagement and offer creative possibility to counteract the 'Hawthorne effect' (Franke and Kaual 1978) known to plague quantitative study and could also threaten qualitative research.

To explain, experience from this study strongly suggests that biases do not arise only from an investigator's point of view but also from the informants' relation to that particular investigator. The sheer fact that they are conscious of being an informant to a particular researcher (and how they view that researcher) definitely shapes the import of their information. Admittedly, this bias must be recognized and minimized, if not eliminated. Interestingly, this may suggest yet another new mode of triangulation - a triangulation of the 'interaction effect' between informant and investigator. Imagine a study where there are two sets of investigators, the known and the mole, a comparative analysis of the evidence gather from the same informants given to these two different sets of inquirers promises to import rich and rare information. How such triangulated prolonged investigation scheme can be effectively and ethically carried out seems a most interesting venue to explore in future research.

Finally, my use of some other validation techniques such as audit trail, peer debriefing, and reflexivity, proved to have helped remedy the limitations substantially. No doubt, a more robust application of these techniques can only increase their benefits. For instance, after the first field interviews and observations, I realized how useful detailed field notes were when human frailty of limited memory confined my coding and theorizing effort. Meticulous audit trail does not only serve audience's review of a study, it also assists researchers in their on-going work. The same applies to debriefing and reflexivity - the more, the better. Last, broader and richer replicated studies are obviously needed to further substantiate and enrich the grounded theory that is the key result of this study. This will no doubt address the theory's generalizability or ex- 
ternal validity, when it is set against a variety of contexts that differ from that from which the theory emerged.

In a nutshell, the limitations in this study confirm yet again one very important research principle: the indispensible value of validation methods and procedures. Possibly, they constitute the best medicine to prevent potential pitfalls and remedy past errors. Again, the opportunity that could be squeezed out of limitations in this study is an aspiration to further develop and advance validation methods and procedures as well as to continue the legacy of the study in the road ahead.

$\mathrm{H}$

UMBLY, I HOPE THIS STUDY, IN SPITE AND BECAUSE OF ITS LIMITATIONS, HAS HELPED UNLOCK A FEW new insights into culture and cultural intelligence. Its contributions and limitations certainly suggest that the road ahead appears even more appealing with many attractive opportunities to pursue. The study thus marks a journey that is itself a destination. 


\section{BIBLIOGRAPHY}

Academy of Management. 2004. Symposium on cultural intelligence at work in the 21st century. Academy of Management Annual Meeting 2004, New Orleans, LA.

Adler, N. J. 1983a. Cross-cultural management research: The ostrich and the trend. Academy of Management Review, 8 (2): 226-232.

Adler, N. J. 1983b. A typology of management studies involving culture. Journal of International Business Studies, 14 (2): 29-47.

Adler, N. J. 2002. International dimensions of organizational behavior. 4th ed. Cincinnati, Ohio: SouthWestern.

Adler, N. J., R. Doktor, and S. G. Redding. 1986. From the Atlantic to the Pacific century: Cross-cultural management reviewed. Journal of Management, 12 (2): 295-318.

Akkerman, S., W. Admiraal, M. Brekelmans, and H. Oost. 2008. Auditing quality of research in social sciences. Quality and Quantity, 42 (2): 257-274.

Allan, G. 2003. A critique of using grounded theory as a research method. Electronic Journal of Business Research Method, 2 (1): 1-10.

Allan, G. 2006. The legitimacy of grounded theory. In Remenyi, D. (Ed), Proceedings of the 5th European conference on research methodology for business and management studies: 1-8. Trinity College, Dublin, Ireland.

Altheide, D. L. and J. M. Johnson. 1994. Criteria for assessing interpretive validity in qualitative research. In Denzin, N. K. and Y. S. Lincoln (Eds), Handbook of qualitative research, 1st ed: 485-499. Thousand Oaks, CA: Sage Publications.

Ambert, A.-M., P. A. Adler, P. Adler, and D. F. Detzner. 1995. Understanding and evaluating qualitative research. Journal of Marriage and the Family, 57: 879-893.

Andrews, T. G. and N. Chompusri. 2001. Lessons in 'cross-vergence': Restructuring the Thai subsidiary corporation. Journal of International Business Studies, 32 (1): 77-93.

Ang, S. and L. Van Dyne (Eds). 2008. Handbook of cultural intelligence: Theory, measurement, and applications. New York: M.E. Sharpe Inc.

Ang, S., L. Van Dyne, and C. Koh. 2006. Personality correlates of the four-factor model of cultural intelligence. Group Organization Management, 31 (1): 100-123.

Ang, S., L. Van Dyne, C. Koh, K. Y. Ng, K. J. Templer, C. Tay, and N. A. Chandrasekar. 2007. Cultural intelligence: Its measurement and effects on cultural judgment and decision making, cultural adaptation and task performance. Management and Organization Review, 3(3): 335-371.

Ang, S., L. Van Dyne, K. Y. Ng, and C. Koh. 2004. The measurement of cultural intelligence, Symposium on Cultural Intelligence at Work in the 21st Century, Academy of Management Meeting, 2004. New Orleans, LA.

Angen, M. J. 2000. Evaluating interpretive inquiry: Reviewing the validity debate and opening the dialogue. Qualitative Health Research, 10 (3): 378-395.

Annells, M. 1996. Grounded theory method: Philosophical perspectives, paradigm of inquiry, and postmodernism. Qualitative Health Research, 6 (3): 379-393.

Annells, M. 1997a. Grounded theory method, part I: Within the five moments of qualitative research. Nursing Inquiry, 4 (3): 120-129.

Annells, M. 1997b. Grounded theory method, part II: Options for users of the method. Nursing Inquiry, 4 (3): 176-180.

Ashforth, B. E. and F. Mael. 1989. Social identity theory and the organization. Academy of Management Review, 14 (1): 20-39.

Atherton, J. S. 2008. Doceo: Shame-culture and guilt-culture. http://www.doceo.co.uk/background/shame_guilt.htm. Accessed 20 September 2008.

Atmiyanandana, V. and J. Lawler. 2004. Culture and management in Thailand. In Warner, M. and P. Joynt (Eds), Culture and management in Asia: 229-248. London: Routledge.

Ayres, L. 2007. Qualitative research proposals-part III: Sampling and data collection. Journal of Wound, Ostomy and Continence Nursing, 34 (3): 242-244. 
Babchuk, W. 1997. Glaser or Strauss? Grounded theory and adult education. Paper presented at Midwest Research-to-Practice Conference in Adult, Continuing and Community Education, East Lansing, Michigan: Michigan State University.

Baker, S. and M. Hess. 2001. Changing concepts of work in Thailand. Journal of Comparative International Management, 4 (1): 33-52.

Bank of Thailand. 2007. Economic data. Bangkok: Bank of Thailand.

Bar-On, R. and J. D. A. Parker (Eds). 2000. The handbook of emotional intelligence: Theory, development, assessment, and application at home, school, and in the workplace. San Francisco: Jossey-Bass.

Bartlett, C., S. Ghoshal, and J. Birkinshaw. 2003. Transnational management: Text and cases. 4th ed. New York: McGraw-Hill/Irwin.

Becker, H. S. 1992. Cases, causes, conjunctures, stories, and imagery. In Ragin, C. and H. S. Becker (Eds), What is a case? Exploring the foundations of social inquiry: 205-216. New York and Cambridge: Cambridge University Press.

Becker, P. H. 1993. Common pitfalls in published grounded theory research. Qualitative Health Research, 3 : 254-260.

Benedict, R. F. 1932. Configurations of culture in North America. American Anthropologist, 34 (1): 1-27.

Benedict, R. F. 1959. Patterns of culture. Boston: Houghton Mifflin.

Benedict, R. F. 1967. The chrysanthemum and the sword: Patterns of Japanese culture. London: Routledge and Kegan Paul.

Bhawuk, D. P. S. and R. W. Brislin. 2000. Cross-cultural training: A review. Applied Psychology: An International Review, 49 (1): 162-191.

Black, J. S. and M. Mendenhall. 1990. Cross-cultural training effectiveness: A review and a theoretical framework for future research. Academy of Management Review, 15 (1): 113-136.

Boisot, M. 2002. The creation and sharing of knowledge. In Choo, C. W. and N. Bontis (Eds), The strategic management of intellectual capital and organizational knowledge: 65-77. Oxford: Oxford University Press.

Bond, M. H., K. Leung, A. Au, K.-K. Tong, S. R. d. Carrasquel, F. Murakami, S. Yamaguchi, G. Bierbrauer, T. M. Singelis, M. Broer, F. Boen, S. M. Lambert, M. C. Ferreira, K. A. Noels, J. v. Bavel, S. Safdar, J. Zhang, L. Chen, I. Solcova, I. Stetovska, T. Niit, K.-K. Niit, H. Hurme, M. Böling, V. Franchi, G. Magradze, N. Javakhishvili, K. Boehnke, E. Klinger, X. Huang, M. Fulop, M. Berkics, P. Panagiotopoulou, S. Sriram, N. Chaudhary, A. Ghosh, N. Vohra, D. F. Iqbal, J. Kurman, R. D. Thein, A. L. Comunian, Son, K. Ae, I. Austers, C. Harb, J. O. T. Odusanya, Z. A. Ahmed, R. Ismail, F. v. deVijver, C. Ward, A. Mogaji, D. L. Sam, M. J. Z. Khan, W. E. Cabanillas, L. Sycip, F. Neto, R. Cabecinhas, P. Xavier, M. Dinca, N. Lebedeva, A. Viskochil, O. Ponomareva, S. M. Burgess, L. Oceja, S. Campo, K.-K. Hwang, J. B. D’souza, B. Ataca, A. Furnham, and J. R. Lewis. 2004. Culture-level dimensions of social axioms and their correlates across 41 cultures. Journal of Cross-Cultural Psychology, 35(5): 548-570.

Boyacigiller, N. A. and N. J. Adler. 1991. The parochial dinosaur: The organizational sciences in a global context. Academy of Management Review, 16 (2): 262-291.

Boyacigiller, N. A., M. J. Kleinberg, M. E. Phillips, and S. A. Sackmann. 2004. Conceptualizing culture: Elucidating the streams of research in international cross-cultural management. In Punnett, B. J. and O. Shenkar (Eds), Handbook for international management research. 2nd ed: 99-167. Ann Arbor: The University of Michigan Press.

Boychuk Duchscher, J. E. and D. Morgan. 2004. Grounded theory: Reflections on the emergence vs. forcing debate. Journal of Advanced Nursing, 48 (6): 605-612.

Boyle, D. P., L. Nackerud, and A. Kilpatrick. 1999. The road less travelled: Cross-cultural, international experiential learning. International Social Work, 42 (2): 201-214.

Braun, W. and M. Warner. 2002. The 'culture-free' versus 'culture specific' management debate. In Warner, M. and P. Joynt (Eds), Managing across cultures: Issues and perspectives. 2nd ed: 13-25. London: International Thomson Business Press.

Brown, J. S. and P. Duguid. 1991. Organizational learning and communities-of-practice: Toward a unified view of working, learning, and innovating. Organization Science, 2 (1): 40.

Brummelhuis, H. t. 1987. Merchant, courtier and diplomat: A history of the contacts between the Netherlands and Thailand. Lochem-Gent: Uitgeversmaatschappij de Tijdstroom.

Bryant, A. 2002. Re-grounding grounded theory. Journal of Information Technology Theory and Application, 4 (1): 25-42.

Bryman, A. and E. Bell. 2003. Business research methods. Oxford: Oxford University Press.

Burke, P. J. 2003. Relationships among multiple identities. In Burke, P. J., T. J. Owens, R. T. Serpe, and P. A. Thoits (Eds), Advances in identity theory and research: 195-214. New York: Kluwer-Plenum. 
Burke, P. J. 2006. Identity change. Social Psychology Quarterly, 69: 81-96.

Byrne, M. 2001. Sampling for qualitative research. AORN, 73 (2): 494-498.

Chang, H. V. 2008. Autoethnography as method. Walnut Creek, CA: Left Coast Press.

Chang, L. (Ed). 2006. Wisdom for the soul: Five millennia of prescriptions for spiritual healing. Washington D.C: Gnosophia Publishers.

Chao, G. T. and H. Moon. 2005. The cultural mosaic: A metatheory for understanding the complexity of culture. Journal of Applied Psychology, 90 (6): 1128-1140.

Charmaz, K. 2000. Grounded theory: Objectivist and constructivist methods. In Denzin, N. K. and Y. S. Lincoln (Eds), Handbook of qualitative research. 2nd ed: 509-535. Thousand Oaks, CA: Sage Publications.

Charmaz, K. 2005. Grounded theory in the 21st century: Applications for advancing social justice studies. In Denzin, N. K. and Y. S. Lincoln (Eds), The Sage handbook of qualitative research. 3rd ed: 507-535. Thousand Oaks, CA: Sage Publications.

Chartrand, T. L. and J. A. Bargh. 1999. The chameleon effect: The perception-behavior link and social interaction. Journal of Personality and Social Psychology, 76 (6): 893-910.

Chenitz, W. C. and J. M. Swanson (Eds). 1986. From practice to grounded theory: Qualitative research in nursing. Menlo Park, CA: Addison-Wesley.

Chiovitti, R. F. and N. Piran. 2003. Rigour and grounded theory research. Journal of Advanced Nursing, 44 (4): 427-435.

Chiva, R. and J. Alegre. 2005. Organizational learning and organizational knowledge: Towards the integration of two approaches. Management Learning, 36 (1): 49-68.

Cohen, D. J. and B. F. Crabtree. 2006. Qualitative research guidelines project. http://www.qualres.net/index.html. Accessed July 2006.

Cohen, D. J. and B. F. Crabtree. 2008. Evaluative criteria for qualitative research in health care: Controversies and recommendations. Annals of Family Medicine, 6 (4): 331-339.

Cook, S. D. N. and J. S. Brown. 1999. Bridging epistemologies: The generative dance between organizational knowledge and organizational knowing. Organization Science, 10 (4): 381-400.

Cooper, R. 2004. Thais mean business: The expat's guide to doing business in Thailand. Singapore: Marshall Cavendish.

Cooper, S. K. 2002. Chameleon, National Geographic. http://magma.nationalgeographic.com/ngexplorer/0210/articles/mainarticle.html. Accessed 20 February 2008.

Corbin, J. and A. L. Strauss. 1990. Grounded theory research: Procedures, canons, and evaluative criteria. Qualitative Sociology, 13 (1): 3-21.

Cornelissen, J. P. 2005. Beyond compare: Metaphor in organization theory. Academy of Management Review, 30 (4): 751-764.

Cornelissen, J. P. 2006a. Metaphor in organization theory: Progress and the past. Academy of Management Review, 31 (2): 485-488.

Cornelissen, J. P. 2006b. Making sense of theory construction: Metaphor and disciplined imagination. Organization Studies (01708406), 27 (11): 1579-1597.

Cornelissen, J. P., C. Oswick, L. Thoger Christensen, and N. Phillips. 2008. Metaphor in organizational research: Context, modalities and implications for research introduction. Organization Studies, 29 (1): 7 22.

Cornelissen, J. P. and M. Kafouros. 2008. Metaphors and theory building in organization theory: What determines the impact of a metaphor on theory? British Journal of Management, 19(4): 365-379.

Cornwell, J. (Ed). 2004. Explanations: Styles of explanation in science. Oxford: Oxford University Press.

Coyne, I. T. 1997. Sampling in qualitative research. Purposeful and theoretical sampling; merging or clear boundaries? Journal of Advanced Nursing, 26 (3): 623-630.

Creighton, M. R. 1990. Revisiting shame and guilt cultures: A forty-year pilgrimage. Ethos, 18 (3): $279-307$.

Creswell, J. W. 1998. Qualitative inquiry and research design: Choosing among five traditions. Thousand Oaks, CA: Sage Publications.

Creswell, J. W. 2002. Educational research: Planning, conducting, and evaluating quantitative and qualitative research. 6th ed. Upper Saddle River, N.J.: Pearson Education.

Creswell, J. W. 2003. Research design: Qualitative, quantitative and mixed method approaches. 2nd ed. Thousand Oaks, CA: Sage Publications.

Creswell, J. W. and R. C. Maietta. 2002. Part 4: Qualitative research. In Miller, D. C. and N. J. Salkind (Eds), Handbook of research design and social measurement. 6th ed: 143-200. Thousand Oaks, CA: Sage Publications.

Creswell, J. W. and D. L. Miller. 2000. Determining validity in qualitative inquiry. Theory Into Practice, 39 (3): $124-130$. 
Crotty, M. 1998. The foundations of social research: Meaning and perspective in the research process. London: Sage Publications.

Curtis, S., W. Gesler, G. Smith, and S. Washburn. 2000. Approaches to sampling and case selection in qualitative research: Examples in the geography of health. Social Science and Medicine, 50 (7-8): 1001-1014.

Cutcliffe, J. R. and H. P. McKenna. 2004. Expert qualitative researchers and the use of audit trails. Journal of Advanced Nursing, 45 (2): 126-133.

David, M. (Ed). 2006. Case study research. London: Sage Publications.

Davidson, J. E. and C. L. Downing. 2000. Contemporary models of intelligence. In Sternberg, R. J. (Ed), Handbook of intelligence: Theories, measurements, and applications: 34-49. Cambridge: Cambridge University Press.

Davies, D. and J. Dodd. 2002. Qualitative research and the question of rigor. Qualitative Health Research, 12 (2): 279-289.

Davis, B., D. J. Sumara, and R. Luce-Kapler. 2000. Engaging minds: Learning and teaching in a complex world. Mahwah, New Jersey: Lawrence Erlbaum.

Denzin, N. K. and Y. S. Lincoln (Eds). 2003. Collecting and interpreting qualitative materials. 2nd ed. Thousand Oaks, CA: Sage Publications.

Denzin, N. K. and Y. S. Lincoln. 2005a. Introduction: The discipline and practice of qualitative research. In Denzin, N. K. and Y. S. Lincoln (Eds), The Sage handbook of qualitative research. 3rd ed: 1-32. Thousand Oaks, CA: Sage Publications.

Denzin, N. K. and Y. S. Lincoln (Eds). 2005b. The Sage handbook of qualitative research. 3rd ed. Thousand Oaks, CA: Sage Publications.

Dey, I. 1993. Qualitative data analysis: A user-friendly guide for social scientists. London: Routledge.

Dey, I. 1999. Grounding grounded theory: Guidelines for qualitative inquiry. San Diego: Academic Press.

Dierkes, M., A. B. Antal, J. Child, and I. Nonaka (Eds). 2001. Handbook of organizational learning and knowledge. Oxford: Oxford University Press.

Dodds, E. R. 1963. The Greeks and the irrational. Berkeley, CA: University of California Press.

Dooley, L. M. 2002. Case study research and theory building. Advances in Developing Human Resources, 4 (3): 335-354.

Dörner, D. 1997. The logic of failure: Recognizing and avoiding error in complex situations. 1st translated ed. Cambridge, Massachusetts: Perseus Press.

Douglas, D. 2003a. Grounded theories of management: A methodological review. Management Research News, 26 (5): 44-52.

Douglas, D. 2003b. Inductive theory generation: A grounded approach to business inquiry. Electronic Journal of Business Research Method, 2 (1): 47-54.

Draucker, C. B., D. S. Martsolf, R. Ross, and T. B. Rusk. 2007. Theoretical sampling and category development in grounded theory. Qualitative Health Research, 17 (8): 1137-1148.

Dubin, R. 1976. Theory building in applied areas. In Dunnette, M. D. (Ed), Handbook of industrial and organizational psychology: 17-39. Chicago: Rand McNally.

Dyer, W. G., Jr. and A. L. Wilkins. 1991. Better stories, not better constructs, to generate better theory: A rejoinder to eisenhardt. Academy of Management Review, 16 (3): 613-619.

Earley, P. C. 1987. Intercultural training for managers: A comparison of documentary and interpersonal methods. Academy of Management Journal, 30 (4): 685-698.

Earley, P. C. 1999. Playing follow the leader: Status-determining traits in relation to collective efficacy across cultures. Organizational Behavior and Human Decision Processes, 80 (3): 192.

Earley, P. C. 2006. Leading cultural research in the future: A matter of paradigms and taste. Journal of International Business Studies, 37: 922-931.

Earley, P. C. and S. Ang. 2003. Cultural intelligence: Individual interactions across cultures. Stanford, California: Stanford University Press.

Earley, P. C. and E. Mosakowski. 2004. Cultural intelligence. Harvard Business Review, 82 (10): 139-146.

Earley, P. C. and H. Singh. 1995. International and intercultural management research: What's next? Academy of Management Journal, 38 (2): 327-340.

Earley, P. C. and R. S. Peterson. 2004. The elusive cultural chameleon: Cultural intelligence as a new approach to intercultural training for the global manager. Academy of Management Learning and Education, 3 (1): 100.

Eisenhardt, K. M. 1989. Building theories from case study research. Academy of Management Review, 14 (4): 532-550.

Eisenhardt, K. M. 1991. Better stories and better constructs: The case for rigor and comparative logic. Academy of Management Review, 16 (3): 620-627. 
Eisenhardt, K. M. and M. E. Graebner. 2007. Theory building from cases: Opportunities and challenges. Academy of Management Journal, 50 (1): 25-32.

Ellis, C. and A. P. Bochner. 2000. Autoethnography, personal narrative, reflexivity: Researcher as subject. In Denzin, N. K. and Y. S. Lincoln (Eds), Handbook of qualitative research. 2nd ed: 733-768. Thousand Oaks, CA: Sage Publications.

Elster, J. 1983. Explaining technical change: A case study in the philosophy of science. Cambridge: Cambridge University Press.

Emden, C. and M. Sandelowski. 1998. The good, the bad and the relative, part one: Conceptions of goodness in qualitative research. International Journal of Nursing Practice, 4 (4): 206-212.

Emden, C. and M. Sandelowski. 1999. The good, the bad and the relative, part two: Goodness and the criterion problem in qualitative research. International Journal of Nursing Practice, 5 (1): 2-7.

Falk, I. and J. Guenther. 2007. Generalising from qualitative research: Case studies from vet in contexts. Paper presented at Evolution, Revolution or Status Quo? The new context for VET, AVETRA 2007 10th Annual Conference. Footscray Park: Victoria University.

Fassinger, R. E. 2005. Paradigms, praxis, problems, and promise: Grounded theory in counseling psychology research. Journal of Counseling Psychology, 52 (2): 156-166.

Feagin, J. R., A. M. Orum, and G. Sjoberg (Eds). 1991. A case for the case study. Chapel Hill: University of North Carolina Press.

Fendt, J. and W. Sachs. 2008. Grounded theory method in management research. Organizational Research Methods, 11 (3): 430-455.

Fiol, C. M. and M. A. Lyles. 1985. Organizational learning. Academy of Management Review, 10 (4): 803-813.

Fitzner, K. 2007. Reliability and validity: A quick review. The Diabetes Educator, 33 (5): 775-780.

Flick, U., E. von Kardorff, and I. Steinke (Eds). 2004. A companion to qualitative research. Thousand Oaks, CA: Sage Publications.

Flyvbjerg, B. 2006. Five misunderstandings about case-study research. Qualitative Inquiry, 12 (2): 219-245.

Fox-Wolfgramm, S. J. 1997. Towards developing a methodology for doing qualitative research: The dynamiccomparative case study method. Scandinavian Journal of Management, 13 (4): 439-455.

Franke, R.H. and Kaul, J.D. 1978. The Hawthorne experiments: First statistical interpretation. American Sociological Review, 43: 623-643.

French, R. 2000. 'Negative capability', 'dispersal' and the containment of emotion. Bristol Business School Teaching and Research Review (3). http://www.u.w.e.ac.uk/ebs/trr/ls3-covt.html. Accessed January 2008.

French, R. 2001. 'Negative capability': Managing the confusing uncertainties of change. Journal of Organizational Change Management, 14 (5): 480-492.

French, W. L. and C. H. Bell. 1972. Organization development: Behavioral science interventions for organization improvement. 1st ed. New Jersey: Prentice Hall.

French, W. L. and C. H. Bell. 1999. Organization development: Behavioral science interventions for organization improvement. 6th ed. New Jersey: Prentice Hall.

Funke, J. 1991. Solving complex problems: Exploration and control of complex systems. In Sternberg, R. J. and P. A. Frensch (Eds), Complex problem solving: Principles and mechanisms: 185-222. Hillsdale, NJ: Lawrence Erlbaum.

Gardner, H., M. L. Kornhaber, and W. K. Wake. 1996. Intelligence: Multiple perspectives. Fort Worth: Harcourt Brace College Publishers.

Garfinkel, A. 1981. Forms of explanation: Rethinking the questions in social theory. New Haven and London: Yale University Press.

Geertz, C. 1993. The interpretation of cultures: Selected essays. London: Fontana Press.

Gelfand, M. J., L. Imai, and R. Fehr. 2008. Thinking intelligently about cultural intelligence. In Ang, S. and L. Van Dyne (Eds), Handbook of cultural intelligence: Theory, measurement, and applications: 375-387. New York: M.E. Sharpe Inc.

Gelfand, M. J., M. Erez, and Z. Aycan. 2007. Cross-cultural organizational behavior. Annual Review of Psychology, 58 (1): 479-514.

Genoways, T. 2005. On the necessity of negative capability. The Virginia Quarterly Review, 81 (4): 1-3.

George, A. L. and A. Bennett. 2005. Case studies and theory development in the social sciences. Cambridge: MIT Press.

Gephart, R. P. 2004. Qualitative research and the Academy of Management Journal. Academy of Management Journal, 47 (4): 454-462. 
Gephart, R. P. 2003. Grounded theory and the integration of qualitative and quantitative research. In Dansereau, F. and F. J. Yammarino (Eds), Research in multi level issues vol 2: Multi-level issues in organizational behavior and strategy: 113-125. Bingley, UK: Emerald Group Publishing Limited.

Gerring, J. 2004. What is a case study and what is it good for? American Political Science Review, 98 (2): $341-$ 354.

Gerring, J. 2007. Case study research: Principles and practice. New York: Cambridge University Press.

Ghoshal, S. 2005. Bad management theories are destroying good management practices. Academy of Management Learning and Education, 4 (1): 75-91.

Gibbon, J. M. 1938. Canadian mosaic: The making of a northern nation. Toronto: McClelland and Stewart.

Gigerenzer, G., P. M. Todd, and the ABC Research Group (Eds). 1999. Simple heuristics that make us smart. New York: Oxford University Press.

Glaser, B. G. 1978. Theoretical sensitivity: Advances in the methodology of grounded theory. Mill Valley, CA: Sociology Press.

Glaser, B. G. 1992. Emergence vs. forcing: Basics of grounded theory analysis. Mill Valley, CA: Sociology Press.

Glaser, B. G. 1998. Doing grounded theory: Issues and discussions. Mill Valley, CA: Sociology Press.

Glaser, B. G. and A. L. Strauss. 1965. Awareness of dying. Chicago: Aldine Publishing Company.

Glaser, B. G. and A. L. Strauss. 1967. The discovery of grounded theory: Strategies for qualitative research. Chicago: Aldine Publishing Company.

Gobo, G. 2008. Re-conceptualizing generalization in qualitative research: Old issues in a new frame. In Alasuutari, P., L. Bickman, and J. Brannen (Eds), The Sage handbook of social research methods: $193-$ 213. Thousand Oaks, CA: Sage Publications.

Golafshani, N. 2003. Understanding reliability and validity in qualitative research. The Qualitative Report, 8 (4): 597-607.

Gomm, R., M. Hammersley, and P. Foster (Eds). 2000. Case study method: Key issues, key texts. Thousand Oaks, CA: Sage Publications.

Goulding, C. 1998. Grounded theory: The missing methodology on the interpretivist agenda. Qualitative Market Research: An International Journal, 1 (1): 50-57.

Goulding, C. 1999. Consumer research, interpretive paradigms and methodological ambiguities. European Journal of Marketing, 33 (9): 859-873.

Goulding, C. 2001. Grounded theory: A magical formula or potential nightmare. The Marketing Review, 2 : 21-34.

Goulding, C. 2002. Grounded theory: A practical guide for management, business and market researchers. London: Sage Publications.

Grant, D. and C. Oswick (Eds). 1996. Metaphor and organizations. London: Sage Publications.

Group \& Organization Management. 2006. Special issue on cultural intelligence, 31 (1).

Grotzer, T. A. and D. N. Perkins. 2000. Teaching intelligence: A performance conception. In Sternberg, Robert J., editor, Handbook of intelligence: Theories, measurements, and applications: 492-515. Cambridge: Cambridge University Press.

Guba, E. G. and Y. S. Lincoln. 1994. Competing paradigms in qualitative research. In Denzin, N. K. and Y. S. Lincoln (Eds), Handbook of qualitative research. 1st ed: 105-117. Thousand Oaks, CA: Sage Publications.

Guba, E. G. and Y. S. Lincoln. 2005. Paradigmatic controversies, contradictions, and emerging confluences. In Denzin, N. K. and Y. S. Lincoln (Eds), The Sage handbook of qualitative research. 3rd ed: 191-215. Thousand Oaks, CA: Sage Publications.

Haig, B. D. 1996. Grounded theory as scientific method. Philosophy of Education Year Book. http://www.ed.uiuc.edu/EPS/PES-Yearbook/95_docs/haig.html. Accessed February 2005.

Hall, E. T. 1989. Beyond culture. Reprint edition ed. New York: Anchor Books.

Hall, E. T. 1990a. The hidden dimension. Reprint edition ed. Garden City, New York: Anchor Books.

Hall, E. T. 1990b. The silent language. Reprint edition ed. Garden City, New York: Anchor Books.

Hall, E. T. and M. R. Hall. 1990. Understanding cultural differences. Yarmouth, Maine: Intercultural Press.

Halpern, E. S. 1983. Auditing naturalistic inquiries: The development and application of a model. Unpublished doctoral dissertation, Indiana University.

Hammersley, M. 1987. Some notes on the terms 'validity' and 'reliability'. British Educational Research Journal, 13 (1): 73-81.

Hammersley, M. 1992. What's wrong with ethnography? London: Routledge.

Hammersley, M. 1998. Reading ethnographic research: A critical guide. 2nd ed. London: Longman.

Hammersley, M. 2007. The issue of quality in qualitative research. International Journal of Research \& Method in Education, 30 (3): 287 - 305. 
Hammersley, M. 2008. Assessing validity in social research. In Alasuutari, P., L. Bickman, and J. Brannen (Eds), The Sage handbook of social research methods: 42-53. Thousand Oaks, CA: Sage Publications.

Hampden-Turner, C. and F. Trompenaars. 1993. Self-constructed lands: The Dutch as god's apprentice. In Hampden-Turner, C. and F. Trompenaars (Eds), The seven cultures of capitalism: Value systems for creating wealth in the US, Britain, Japan, Germany, France, Sweden and the Netherlands: 261-292. London: Doubleday.

Hampden-Turner, C. and F. Trompenaars (Eds). 1993. The seven cultures of capitalism: Value systems for creating wealth in the US, Britain, Japan, Germany, France, Sweden and the Netherlands. London: Doubleday.

Handley, K., T. Clark, R. Fincham, and A. Sturdy. 2007. Researching situated learning: Participation, identity and practices in client-consultant relationships. Management Learning, 38 (2): 173-191.

Healy, M. and C. Perry. 2000. Comprehensive criteria to judge validity and reliability of qualitative research within the realism paradigm. Qualitative Market Research: An International Journal, 3 (3): 118-126.

Heath, H. and S. Cowley. 2004. Developing a grounded theory approach: A comparison of Glaser and Strauss. International Journal of Nursing Studies, 41 (2): 141-150.

Hellström, T. 2008. Transferability and naturalistic generalization: New generalizability concepts for social science or old wine in new bottles? Quality and Quantity, 42 (3).

Henry, J. 1989. Meaning and practice in experiential learning. In Weil, S. W. and I. McGill (Eds), Making sense of experiential learning: Diversity in theory and practice: 25-37. Milton Keynes: The Society for Research into Higher Education and Open University Press.

Heracleous, L. and C. D. Jacobs. 2008. Understanding organizations through embodied metaphors. Organization Studies, 29 (1): 45-78.

den Hertog, J. F. 2007. Personal communication.

den Hertog, J.F. and Huizenga. 2000. The knowledge enterprise. London: Imperial College Press.

den Hertog, J. F. and Maarten Verkerk. 2007. The manager as a mole, participant observation by men who manage. In Remenyi, D. (Ed), Proceedings of the 6th European conference on research methodology for business and management studies: 135-142. Lisbon, Portugal: Universidade Nova de Lisboa.

Hofstede, G. 1980. Culture's consequences: International differences in work-related values. 1st ed. Beverly Hills, CA: Sage Publications.

Hofstede, G. 2001. Culture's consequences: Comparing values, behaviors, institutions, and organizations across nations. 2nd ed. London: Sage Publications.

Hofstede, G. and G.- J. Hofstede. 2005. Cultures and organizations: Software of the mind. 2nd ed. New York: McGraw-Hill.

Hofstede, G. and R. R. McCrae. 2004. Personality and culture revisited: Linking traits and dimensions of culture. Cross-Cultural Research, 38 (1): 52-88.

Hofstede, G., B. Neuijen, D. D. Ohayv, and G. Sanders. 1990. Measuring organizational cultures: A qualitative and quantitative study across twenty cases. Administrative Science Quarterly, 35 (2): 286-316.

Hofstede, G.-J. 2008. Personal communication.

Hogg, M. A., D. J. Terry, and K. M. White. 1995. A tale of two theories: A critical comparison of identity theory with social identity theory. Social Psychology Quarterly, 58 (4): 255-269.

Holden, N. J. 2001. Knowledge management: Raising the spectre of the cross-cultural dimension. Knowledge and Process Management, 8 (3): 155-163.

Holden, N. J. 2002. Cross-cultural management: A knowledge management perspective. London: Financial Times/Prentice Hall.

Holmes, H., V. Poonprasit, and S. Komin. 2006. Some cornerstones of Thai culture. Training materials. Bangkok: Cross-Cultural Management Co. Ltd.

Holmes, H. and S. Tangtongtavy. 1997. Working with the Thais. Bangkok: White Lotus.

van der Horst, H. 1996. The low sky: Understanding the Dutch. Schiedam: Scriptum Books.

House, R. J., P. J. Hanges, M. Javidan, P. W. Dorfman, and V. Gupta (Eds). 2004. Culture, leadership, and organizations: The GLOBE study of 62 societies. London: Sage Publications.

Huntington, S. P. 1993. The clash of civilizations? Foreign Affairs, 72 (3): 22-49.

Huntington, S. P. 1996a. The clash of civilizations and the remaking of world order. New York: Simon \& Schuster.

Huntington, S. P. (Ed). 1996b. The clash of civilizations? The debate. New York: Foreign Affairs.

Illeris, K. 2007. What do we actually mean by experiential learning? Human Resource Development Review, 6 (1): 84-95.

Inglehart, R. 1997. Modernization and postmodernization: Cultural, economic, and political change in 43 societies. Princeton, N.J.: Princeton University Press. 
Inglehart, R. and W. E. Baker. 2000. Modernization, cultural change, and the persistence of traditional values. American Sociological Review, 65 (1): 1-50.

Inglehart, R., M. Basanez, J. Diez-Medrano, L. Halman, and R. Luijkx (Eds). 2004. Human beliefs and values: $A$ cross-cultural sourcebook based on the 1999-2002 values surveys. Beunos Aires, Argentina: Siglo Veintiuno Editores.

Inkeles, A. and D. J. Levinson. 1969. National character: The study of modal personality and sociocultural systems. In Lindzey, G. and E. Aronson (Eds), The handbook of social psychology: 418-509. New York: McGraw-Hill.

Inns, D. 2002. Metaphor in the literature of organizational analysis: A preliminary taxonomy and a glimpse at a humanities-based perspective. Organization, 9 (2): 305-330.

Jackson, T. and Z. Aycan. 2001. International journal of cross cultural management-towards the future. International Journal of Cross Cultural Management, 1 (5-9).

Jackson, T. and Z. Aycan. 2006. From cultural values to cross cultural interfaces. International Journal of Cross Cultural Management, 6 (1): 5-13.

John A, E. 1981. An overview of the relationships between mimicry and crypsis. Biological Journal of the Linnean Society, 16 (1): 25-31.

Johnson, R. B. 1997. Examining the validity structure of qualitative research. Education, 118 (3): $282-292$.

Johnston, J. M. and H. S. Pennypacker. 1980. Strategies and tactics of human behavioural research. Hillsdale, NJ: Lawrence Erlbaum.

Kanungo, R. P. 2006. Cross culture and business practice: Are they coterminous or cross-verging? Cross Cultural Management: An International Journal, 13 (1): 23-31.

Kaplan, D. 2004. The Sage handbook of quantitative methodology for the social sciences. Thousand Oaks, CA: Sage Publications.

Keats, J. and H. B. E. Forman (Ed). 2004. The letters of John Keats. Reprinted ed. London: Kessinger Publishing.

Keddy, B., S. L. Sims, and P. N. Stern. 1996. Grounded theory as feminist research methodology. Journal of Advanced Nursing, 23 (3): 448-453.

Kelle, U. 2005. "Emergence" vs. "forcing" of empirical data? A crucial problem of "grounded theory" reconsidered. Forum Qualitative Sozialforschung / Forum: Qualitative Social Research, 6 (2). http://www.qualitative-research.net/index.php/fqs/article/view/467. Accessed July 2006.

Kemmis, S. 1980. The imagination of the case and the invention of the study. In Simons, H. (Ed), Towards a science of the singular: Essays about case study in educational research and evaluation: 96-142. Norwich: Care Occasional Publications: University of East Anglia, Centre for Applied Research in Education.

Kihlstrom, J. F. and N. Cantor. 2000. Social intelligence. In Sternberg, R. J. (Ed), Handbook of intelligence: Theories, measurements, and applications: 359-379. Cambridge: Cambridge University Press.

Kinach, B. M. 1996. Grounded theory as scientific method: Haig-inspired reflections on educational research methodology. http://www.ed.uiuc.edu/EPS/PES-Yearbook/95_docs/kinach.html. Accessed February 2005.

King, P. 1993. The business culture in the Netherlands. In Randlesome, C., B. William, K. Bruton, C. Gordon, and P. King (Eds), Business cultures in Europe: 323-364. Oxford: Butterworth-Heinemann.

Klausner, W. J. 1993. Reflections on Thai culture. 4th ed. Bangkok: Siam Society.

Klinge, K., A. Rohmann, and U. Piontkowski. 2009. Intercultural sensitization with synthetic cultures: Evaluation of a computer-based multimedia learning tool. International Journal of Intercultural Relations, 33 (6): 507-515.

Kluckhohn, C. 1951. Values and value-orientation in the theory of action. In Parsons, T. and E. A. Shils (Eds), Toward a general theory of action: 388-433. New York: Harper and Row.

Kluckhohn, F. R. and F. L. Strodtbeck. 1961. Variations in value orientations. Evanstone, Illinois: Row, Peterson and Company.

Knigge, L. and M. Cope. 2006. Grounded visualization: Integrating the analysis of qualitative and quantitative data through grounded theory and visualization. Environment and Planning A, 38 (11): 2021-2037.

Knox, K. 2004. A researcher's dilemma - philosophical and methodological pluralism. Electronic Journal of Business Research Method, 2 (2): 119-128.

Komin, S. 1989. Social dimensions of industrialization in Thailand. Bamgkok: National Institute of Development Administration (NIDA).

Komin, S. 1991. Psychology of the Thai people: Values and behavioral patterns. Bangkok: National Institute of Development Administration (NIDA). 
Kolb, D. A., R. Boyatzis, and C. Mainemelis. 2000. Experiential learning theory: Previous research and new directions. In Sternberg, Robert J. and L. F. Zhang (Eds), Perspectives on cognitive learning, and thinking styles: 193-210. Mahwah, NJ: Lawrence Erlbaum.

Kroeber, A. L. and C. Kluckhohn. 1963. Culture: A critical review of concepts and definitions. New ed. New York: Random House.

Kübler-Ross, E. 1973. On death and dying. London: Routledge.

Kuzel, A. J. 1999. Sampling in qualitative inquiry. In Crabtree, B. F. and W. L. Miller (Eds), Doing qualitative research. 2nd ed: 33-45. Thousand Oaks, CA: Sage Publications.

Kvale, S. 1995. The social construction of validity. Qualitative Inquiry, 1 (1): 19-40.

Kwanjai, N. N. and J. F. den Hertog. 2009. Multinationals are multicultural units. In Dolfsma, W., G. Duysters, and I. Costa (Eds), Multinationals and emerging economies: The quest for innovation and sustainability: 6-23. London: Edward Elgar Publishing.

Kwanjai, N. N. and J. F. den Hertog. 2010. Portrait of an odd-eyed cat: Cultural crossing as a trademark for a Dutch-Thai strategic alliance. In Ulijn, J., G. Duijsters, and E. Meijer (Eds), Strategic alliances, mergers and acquisitions: The influence of culture on successful cooperation: 227-254. London: Edward Elgar Publishing.

Lakoff, G. and M. Johnson. 1980. Metaphors we live by. Chicago: University of Chicago Press.

Langley, A. 1999. Strategies for theorizing from process data. Academy of Management Review, 24 (4): 691 710.

Larsen, H. H. 2004. Experiential learning as management development: Theoretical perspectives and empirical illustrations. Advances in Developing Human Resources, 6 (4): 486-503.

Lave, J. 1993. The practice of learning. In Chaiklin, S. and J. Lave (Eds), Understanding practice: Perspectives on activity and context: 3-32. Cambridge: Cambridge University Press.

Lave, J. and E. Wenger. 1991. Situated learning: Legitimate peripheral participation. Cambridge: Cambridge University Press.

Lawler, J. and V. Atmiyanandana. 2004. HRM in Thailand: A post-1997 update. In Rowley, C. and J. Benson (Eds), The management of human resources in the Asia Pacific region convergence revisited: 165-185. London: Routledge.

Lawrence, P. A. 1991. Management in the Netherlands. Oxford: Clarendon Press.

Leung, K., R. S. Bhagat, N. R. Buchan, M. Erez, and C. B. Gibson. 2005. Culture and international business: Recent advances and their implications for future research. Journal of International Business Studies, 36 (4): 357-378.

Leung, K. and M. H. Bond. 2004. Social axioms: A model of social beliefs in multi-cultural perspective. In Zanna, M. P. (Ed), Advances in experimental social psychology: 119-197. San Diego, CA: Elsevier Academic Press.

Leung, K., M. H. Bond, S. R. d. Carrasquel, C. Muñoz, M. Hernández, F. Murakami, S. Yamaguchi, G. Bierbrauer, and T. M. Singelis. 2002. Social axioms: The search for universal dimensions of general beliefs about how the world functions. Journal of Cross-Cultural Psychology, 33(3): 286-302.

Levin, J. and J. A. Fox. 2006. Elementary statistics in social research. 10th ed. Boston, MA: Allyn and Bacon.

Lewis, D. 1996a. The organizational culture saga - from OD to TQM: A critical review of the literature. Part 1 - concepts and early trends. Leadership \& Organization Development Journal, 17 (1): 12-19.

Lewis, D. 1996b. The organizational culture saga - from OD to TQM: A critical review of the literature. Part 2 - applications. Leadership \& Organization Development Journal, 17 (1): 9-16.

Lewis, H. R. and C. H. Papadimitriou. 1998. Elements of the theory of computation. Upper Saddle River, NJ: Prentice Hall.

Lewis, J. and J. Ritchie. 2003. Generalising from qualitative research. In Ritchie, J. and J. Lewis (Eds), Qualitative research practice: 263-286. London: Sage Publications.

Lincoln, Y. S. 1995. Emerging criteria for quality in qualitative and interpretive research. Qualitative Inquiry, 1 (3): 275-289.

Lincoln, Y. S. and E. G. Guba. 1985. Naturalistic inquiry. Thousand Oaks, CA: Sage Publications.

Lincoln, Y. S. and E. G. Guba. 2000. Paradigmatic controversies, contradictions, and emerging confluences. In Denzin, N. K., Y. S. Lincoln, and E. G. Guba (Eds), Handbook of qualitative research. 2nd ed.: 163-188 Thousand Oaks, CA: Sage Publications.

Little, D. 1991. Varieties of social explanation: An introduction to the philosophy of social science. Boulder, Calorado: Westview Press.

Locke, K. 1996. Rewriting the discovery of grounded theory after 25 years? Journal of Management Inquiry, 5 (3): 239-245.

Locke, K. 2001. Grounded theory in management research. London: Sage Publications. 
Long, T. and M. Johnson. 2000. Rigour, reliability and validity in qualitative research. Clinical Effectiveness in Nursing, 4 (1): 30.

Lösch, A. 2006. Combining quantitative methods and grounded theory for researching e-reverse auctions. Libri, 56: 133-144.

Lowell, A. L. 1934. At war with academic traditions in America. Cambridge: Harvard University Press.

Luborsky, M. R. and R. L. Rubinstein. 1995. Sampling in qualitative research: Rationale, issues, and methods. Research on Aging, 17 (1): 89-113.

van Maanen, J. 1979. The fact of fiction in organizational ethnography. Administrative Science Quarterly, 24 (4): $539-550$

van Maanen, J. 1988. Tales of the field: On writing ethnography. Chicago: University of Chicago Press.

MacDonald, B. and R. Walker. 1975. Case study and the social philosophy of educational research. Cambridge Journal of Education, 5 (1): 2-11.

Mackintosh, N. J. 1998. IQ and human intelligence. Oxford: Oxford University Press.

Mainemelis, C., R. E. Boyatzis, and D. A. Kolb. 2002. Learning styles and adaptive flexibility: Testing experiential learning theory. Management Learning, 33 (1): 5-33.

Malterud, K. 2001. Qualitative research: Standards, challenges, and guidelines. The Lancet, 358 (9280): 483488.

Marlowe, H. A. 1986. Social intelligence: Evidence for multidimensionality and construct independence. Journal of Educational Psychology, 78 (1): 52-58.

Marshall, M. N. 1996. Sampling for qualitative research. Family Practice, 13 (6): 522-526.

Martin, P. Y. and B. A. Turner. 1986. Grounded theory and organizational research. Journal of Applied Behavioral Science, 22 (2): 141-157.

Mason, J. 2002. Qualitative researching. 2nd ed. London: Sage Publications.

Mauthner, N. S. and A. Doucet. 2003. Reflexive accounts and accounts of reflexivity in qualitative data analysis. Sociology, 37 (3): 413-431.

Maxwell, J. A. 1992. Understanding and validity in qualitative research. Harvard educational review, 62 (3): 279-300.

Mayer, J. D., P. Slovey, and D. Caruso. 2000. Models of emotional intelligence. In Sternberg, R. J., editor, Handbook of intelligence: Theories, measurements, and applications: 396-420. Cambridge: Cambridge University Press.

Mays, N. and C. Pope. 1995. Qualitative research: Rigour and qualitative research. British Medical Journal (BMJ), 311 (6997): 109-112.

Mays, N. and C. Pope. 2000. Qualitative research in health care: Assessing quality in qualitative research. British Medical Journal (BMJ), 320 (7226): 50-52.

Maznevski, M. L., C. B. Gomez, J. J. DiStefano, N. G. Noorderhaven, and P.-C. Wu. 2002. Cultural dimensions at the individual level of analysis: The cultural orientations framework. International Journal of Cross Cultural Management, 2 (3): 275-295.

McCaig, N. M. 1996. Understanding global nomads. In Smith, C. D., editor, Strangers at home: 99-120. New York: Aletheia.

McLuhan, M. 1964. Understanding media: The extensions of men. New York: McGraw-Hill.

Mcllveen, P. 2008. Autoethnography as a method for reflexive research and practice in vocational psychology. Australian Journal of Career Development, 17 (2): 13-20.

Mead, M. 1949. Coming of age in Samoa: A psychological study of primitive youth for western civilisation. Reprinted. ed. New York: New American Library.

Mead, M. 1983. Cultural discontinuities and personality transformation. Journal of Social Issues, 39 (4): 161177.

Merriam, S. B. 1995. What can you tell from an $\mathrm{N}$ of 1 ? Issues of validity and reliability in qualitative research. PAACE Journal of Lifelong Learning, 4: 51-60.

Merriam, S. B., R. S. Caffarella, and L. M. Baumgartner. 2007. Learning in adulthood: A comprehensive guide. 3rd ed. San Francisco: Wiley-Interscience.

Merta, R. J., E. M. Stringham, and J. G. Ponterotto. 1988. Simulating culture shock in counselor trainees: An experiential exercise for cross-cultural training. Journal of Counseling \& Development, 66 (5): 242-245.

Meyer, M. 2007. The Meyer literature site: Glossary of literary terms. http://www.bedfordstmartins.com/literature/bedlit/glossary_t.htm. Accessed March 2007.

Michalewicz, Z. and D. B. Fogel. 2000. How to solve it: Modern heuristics. Berlin: Springer.

Miles, M. B. and A. M. Huberman. 1994. Qualitative data analysis: An expanded sourcebook. 2nd ed. Thousand Oaks, CA: Sage Publications. 
Miller, D. L. 1997. One strategy for assessing the trustworthiness of qualitative research: Operationalizing the external audit. Paper presented at The Annual Meeting of the American Educational Research Association, Chicago, IL.

Mirvis, P. H. and P. H. Mirvis. 1996. Historical foundations of organization learning. Journal of Organizational Change Management, 9 (1): 13-31.

Mitchell, J. C. 1983. Case and situational analysis. Sociological Review, 31 (2): 187-211.

Morgan, G. 1986. Images of organizations. 1st ed. Beverly Hills: Sage Publications.

Morgan, G. 2006. Images of organizations. 2nd and updated ed. Beverly Hills: Sage Publications.

Morse, J. M. 1995. The significance of saturation. Qualitative Health Research, 5 (2): 147-149.

Morse, J. M. 2000. Determining sample size. Qualitative health research. Qualitative Health Research, 10 (1): 3-5.

Mulder, N. 2000. Inside Thai society: Religion, everyday life, change. Chiang Mai: Thailand: Silkworm Books.

Murdock, G. P. 1940. The cross-cultural survey. American Sociological Review, 5 (3): 361-370.

Murdock, G. P. and C. Provost. 1973. Measurement of cultural complexity. Ethnology, 12 (4): 379-392.

Naumes, W. and M. Naumes. 2006. The art and craft of case writing. 2nd ed. New York: M.E. Sharpe Inc.

Neisser, U. 1976. General, academic, and artificial intelligence. In Resnick, L., editor, The nature of intelligence. Hillsdale, NJ: Lawrence Erlbaum Associate.

Neuman, W. L. 2003. Social research method: Qualitative and quantitative approaches. 5 ed. Boston: Allyn and Bacon.

Ng, K.-Y. and P. C. Earley. 2006. Culture + intelligence: Old constructs, new frontiers. Group Organization Management, 31 (1): 4-19.

Nonaka, I. 1991. The knowledge-creating company. Harvard Business Review, 69 (12): 96-104.

Nonaka, I. 1994. A dynamic theory of organizational knowledge creation. Organization Science, 5 (1): 14-37.

Nonaka, I. and H. Takeuchi. 1995. The knowledge-creating company: How Japanese companies create the dynamics of innovation. Oxford: Oxford University Press.

Orum, A. M., J. R. Feagin, and G. Sjoberg. 1991. Introduction: The nature of the case study. In Feagin, J. R., A. M. Orum, and G. Sjoberg (Eds), A case for the case study: 1-26. Chapel Hill: University of North Carolina Press.

Ou, H. 2009. Situated knowledge: A practice-based view on intercultural interaction. Paper presented at Academy of Management, Chicago, Illinois.

Oxford University Press, editor. 1989. Oxford English dictionary. 2nd ed. Oxford: Oxford University Press.

Partington, D. 2000. Building grounded theories of management action. British Journal of Management, 11 (2): 91-102.

Patton, M. Q. 1999. Enhancing the quality and credibility of qualitative analysis. Health Services Research, 34 (5-2): 1189-1208.

Patton, M. Q. 2001. Qualitative research and evaluation methods. 3rd ed. Thousand Oaks, CA: Sage Publications.

Peterson, B. 2004. Cultural intelligence: A guide to working with people from other cultures. Boston: Intercultural Press.

Peterson, M. F. 2006. Understanding a culturally diverse world. Lecture for the Opening Ceremonies of the Hofstede Chair in Cultural Diversity, Masstricht University. Maastricht, the Netherlands.

Peterson, M. F. and S. L. Castro. 2006. Measurement metrics at aggregate levels of analysis: Implications for organization culture research and the GLOBE project. The Leadership Quarterly, 17 (5): 506-521.

Pitt, J. (Ed). 1988. Theories of explanation. Oxford: Oxford University Press.

Platt, J. 1988. What can case studies do? In Burgess, R. (Ed), Studies in qualitative methodology: 1-23. Greenwich, CT: JAI Press.

Plum, Elisabeth. 2007. Cultural intelligence - a concept for bridging and benefiting from cultural differences. Book abstract, translated and commented by Cunnane, D. From Plum, E., B. Achen, I. Dræby and I. Jensen, Kulturel Intelligens. Copenhagen: Børsens Forlag.

Polanyi, M. 1974. Personal knowledge: Towards a post-critical philosophy. Chicago: University of Chicago Press.

Polanyi, M. 1983. Tacit dimension. London: Peter Smith Publication.

Pollock, D. C. and R. E. van Reken. 2001. The third culture kid experience: Growing up among worlds. London: Nicholas Brealey Publishing/Intercultural Press.

Pólya, G. 1973. How to solve it: A new aspect of mathematical method. 2nd ed. Princeton: Princeton University Press.

Porter, J. 1965. The vertical mosaic: An analysis of social class and power in Canada. Toronto: University of Toronto Press. 
Quay, J. 2003. Experience and participation: Relating theories of learning. The Journal of Experiential Education, 26 (2): 105-112.

Ragin, C. 1992. 'Casing' and the process of social inquiry. In Ragin, C. and H. S. Becker (Eds), What is a case? Exploring the foundations of social inquiry. New York and Cambridge: Cambridge University Press.

Ragin, C. 1992. Introduction: Cases of "what is a case?" In Ragin, C. and H. S. Becker (Eds), What is a case? Exploring the foundations of social inquiry: 217-226. New York and Cambridge: Cambridge University Press.

Ragin, C. and H. S. Becker (Eds). 1992. What is a case? Exploring the foundations of social inquiry. New York and Cambridge: Cambridge University Press.

Redmond, M. 2002. Wandering into Thai culture. Bangkok: Redmondian Insight Enterprises.

Remenyi, D. 2005. Tell me a story - a way to knowledge. The Electronic Journal of Business Research Methodology, 3 (2): 133-140.

Rennie, D. L. 1998. Grounded theory methodology: The pressing need for a coherent logic of justification. Theory and Psychology, 8 (1): 102109.

Richardson, L. 2003. Writing: A method of inquiry. In Denzin, N. K. and Y. S. Lincoln (Eds), Collecting and interpreting qualitative materials. 2nd ed: 499-541. Thousand Oaks, CA: Sage Publications.

Richardson, L. and E. A. St. Pierre. 2005. Writing: A method of inquiry. In Denzin, N. K. and Y. S. Lincoln (Eds), The Sage handbook of qualitative research. 3rd ed: 959-978. Thousand Oaks, CA: Sage Publications.

Ricks, D. A. 1985. International business research: Past, present, and future. Journal of International Business Studies, 16 (2): 1-4.

Roach, J. 2005. Chameleons say it with color. National Geographic News. http://news.nationalgeographic.com/news/2005/09/0926_050926_chameleon.html. Accessed December 2008.

Roberts, K. H. 1970. On looking at an elephant: An evaluation of cross-cultural research related to organizations. Psychological Bulletin (74): 327-350.

Robrecht, L. C. 1995. Grounded theory: Evolving methods. Qualitative Health Research, 5 (2): 169-177.

Rodgers, B. L. and K. V. Cowles. 1993. The qualitative research audit trail: A complex collection of documentation. Research in Nursing and Health, 16 (2): 219-226.

Rokeach, M. 1968. Beliefs, attitudes, and values: A theory of organization and change. San Francisco: JosseyBass.

Rokeach, M. 1973. The nature of human values. New York: The Free Press.

Rowley, C. 1998. Introduction: Comparisons and perspectives on HRM in the Asia Pacific. In Rowley, C. (Ed), Human resource management in the Asia Pacific region: Convergence questioned: 1-18. London: Frank Cass.

Ryle, G. 1949. The concept of mind. London: Hutchinson.

Sackmann, S. A. 1991. Cultural knowledge in organizations: Exploring the collective mind. Newbury Park, CA: Sage Publications.

Salmon, W. C. 1990. Four decades of scientific explanation. Minneapolis: University of Minnesota Press.

Sandelowski, M. 1986. The problem of rigor in qualitative research. Advances in Nursing Science, 8 (3): 27-37.

Sandelowski, M. 1993. Rigor or rigor mortis: The problem of rigor in qualitative research revisited. Advances in Nursing Science, 16 (2): 1-8.

Sandelowski, M. 1995. Sample size in qualitative research. Research in Nursing and Health, 18 (2): $179-183$.

Schein, E. H. 2004. Organizational culture and leadership. 3rd ed. San francisco, CA: Jossey-Bass.

Scherer, A. G. 2003. Modes of explanation in organization theory. In Tsoukas, H. and C. Knudsen (Eds), The oxford handbook of organization theory: 310-344. Oxford: Oxford University Press.

Schneider, S. C. and J.-L. Barsoux. 2003. Managing across cultures. 2nd ed. New York: Prentice Hall.

Schultz, M. and M. J. Hatch. 1996. Living with multiple paradigms: The case of paradigm interplay in organizational culture studies. Academy of Management Review, 21 (2): 529-557.

Schwandt, T. A. 1999. On understanding understanding. Qualitative Inquiry, 5 (4): 451-464.

Schwandt, T. A. 2000. Three epistemological stances for qualitative inquiry: Interpretivism, hermeneutics, and social constructionism. In Denzin, N. K., Y. S. Lincoln, and E. G. Guba (Eds), Handbook of qualitative research. 2nd ed: 292-331. Thousand Oaks, CA: Sage Publications.

Schwartz, J. 1997. "Roots" and "Mosaic" in a Balkan border villiage: Locating cultural production. In Olwig, K. F. and K. Hastrup (Eds), Siting culture: The shifting anthropological object: 255-267. London: Routledge.

Schwartz, S. H. 1992. Universals in the content and structure of values: Theory and empirical tests in 20 countries. In Zanna, M. P. (Ed), Advances in experimental social psychology: 1-65. New York: Academic Press.

Schwartz, S. H. 1999. A theory of cultural values and some implications for work. Applied Psychology: An International Review, 48 (1): 23. 
Schwartz, S. H. and A. Bardi. 2001. Value hierarchies across cultures: Taking a similarities perspective. Journal of Cross-Cultural Psychology, 32: 268-90.

Schwartz, S. H. and L. Sagiv. 1995. Identifying culture-specifics in the content and structure of values. Journal of Cross-Cultural Psychology, 26: 92-116.

Seale, C. and D. Silverman. 1997. Ensuring rigour in qualitative research. European Journal of Public Health, 7 (4): 379-384.

Segaller, D. 2005. Thai ways. Chiang Mai: Silkworm Books.

Selfridge, R. J. and S. L. Sokolik. 1975. A comprehensive view of organizational development. MSU Business Topics, 23 (1): 47-61.

Shenkar, O. 2001. Cultural distance revisited: Towards a more rigorous conceptualization and measurement of cultural differences. Journal of International Business Studies, 32 (3): 519-535.

Shenkar, O. 2004. One more time: International business in a global economy. Journal of International Business Studies, 35 (2): 161-171.

Siengthai, S. and C. Bechter. 2004. Human resource management in Thailand. In Budhwar, P. (Ed), HRM in Southeast Asia and the Pacific rim: 141-172. London: Routledge.

Siengthai, S. and C. Bechter. 2005. Human resource management in Thailand: A strategic transition for firm competitiveness. Research and Practicein Human Resource Management, 13 (1): 18-29.

Silverman, D. (Ed). 2004. Qualitative research: Theory, method and practice. 2nd ed. Thousand Oaks, CA: Sage Publications.

Simons, H., editor. 1980. Towards a science of the singular: Essays about case study in educational research and evaluation. Norwich: Care Occasional Publications: University of East Anglia, Centre for Applied Research in Education.

Simons, H. 1996. The paradox of case study. Cambridge Journal of Education, 26 (2): 225-240.

Simons, H. 2009. Case study research in practice. Thousand Oaks, CA: Sage Publications.

Simpson, P. F., R. French, and C. E. Harvey. 2002. Leadership and negative capability. Human Relations, 55: 1209-1226.

Sluyterman, K. E. 2005. Dutch enterprise in the twentieth century: Business strategies in a small open economy. London: Routledge.

Smaling, A. 2003. Inductive, analogical, and communicative generalization. International Journal of Qualitative Methods, 2 (1): 1-31.

Smith, J. K. 1984. The problem of criteria for judging interpretive inquiry. Educational Evaluation and Policy Analysis, 6 (4): 379-391.

Smith, J. K. and D. K. Deemer. 2000. The problem of criteria in the age of relativism. In Denzin, N. K. and Y. S. Lincoln (Eds), Handbook of qualitative research. 2nd ed: 877-896. Thousand Oaks, CA: Sage Publications.

Smith, P. B. 2001. The end of the beginning? International Journal of Cross Cultural Management, 1 (1): 21 24.

Smith, P. B. 2004. Nations, cultures, and individuals: New perspectives and old dilemmas. Journal of CrossCultural Psychology, 35 (1): 6-12.

Sparkes, A. C. 2001. Myth 94: Qualitative health researchers will agree about validity. Qualitative Health Research, 11 (4): 538-552.

Spencer, L., J. Richie, J. Lewis, and L. Dillon. 2003. Quality in qualitative evaluation: A framework for assessing research evidence. London: National Centre for Social Research.

Spender, J. C. 2008. Organizational learning and knowledge management: Whence and whither? Management Learning, 39 (2): 159-176.

Stake, R. E. 1978. The case study method in social inquiry. Educational Researcher, 7 (2): 5-9.

Stake, R. E. 1995. The art of case study research. Thousand Oaks, CA: Sage Publications.

Stake, R. E. 2000. Case studies. In Denzin, N. K., Y. S. Lincoln, and E. G. Guba (Eds), Handbook of qualitative research. 2nd ed: 435-454. Thousand Oaks, CA: Sage Publications.

Stake, R. E. and D. J. Trumbull. 1982. Naturalistic generalizations. Review Journal of Philosophy and Social Science, 7 (1-2): 1-12.

Starrett, A. 1993. Adaptive resemblance: A unifying concept for mimicry and crypsis. Biological Journal of the Linnean Society, 48 (4): 299.

Steinke, I. 2004. Quality criteria in qualitative research. In Flick, U., E. von Kardorff, and I. Steinke (Eds), $A$ companion to qualitative research: 184-190. Thousand Oaks, CA: Sage Publications.

Stenbacka, C. 2001. Qualitative research requires quality concepts of its own. Management Decision, 39 (7): 551-555.

Sternberg, R. J. 1985. Human intelligence: The model is the message. Science, 230: 1111-1118. 
Sternberg, R. J. 1986. A framework for understanding conceptions of intelligence. In Sternberg, R. J. and D. K. Detterman (Eds), What is intelligence? Contemporary viewpoints on its nature and definition: 3-15. Norwood N.J.: Ablex Publishing.

Sternberg, R. J. 1997. Successful intelligence. New York: Plume.

Sternberg, R. J., editor. 2000. Handbook of intelligence: Theories, measurements, and applications. Cambridge: Cambridge University Press.

Sternberg, R. J. and E. L. Grigorenko. 2006. Cultural intelligence and successful intelligence. Group Organization Management, 31 (1): 27-39.

Stets, J. E. and P. J. Burke. 2000. Identity theory and social identity theory. Social Psychology Quarterly, 63 (3): 224-237.

Strauss, A. L. and J. Corbin. 1990. Basics of qualitative research: Grounded theory procedures and techniques. Newbury Park: Sage Publications.

Strauss, A. L. and J. Corbin. 1994. Grounded theory methodology - an overview. In Denzin, N. K. and Y. S. Lincoln (Eds), Handbook of qualitative research. 1st ed: 273-285. Thousand Oaks, CA: Sage Publications.

Strauss, A. L. and J. Corbin. 1998. Basics of qualitative research: Techniques and procedures for developing grounded theory. 2nd ed. Thousand Oaks, CA: Sage Publications.

Stryker, S. and P. J. Burke. 2000. The past, present and future of identity theory. Social Psychology Quarterly, 63: 284-297.

Stuart-Fox, D. and A. Moussalli. 2008. Selection for social signalling drives the evolution of chameleon colour change. PLoS Biology, 6 (1): e25.

Suddaby, R. 2006. From the editors: What grounded theory is not. Academy of Management Journal, 49 (4): 633-642.

Swanson, J. M. 1986. Analyzing data for categories and description. In Chenitz, W. C. and J. M. Swanson (Eds), From practice to grounded theory: Qualitative research in nursing: 121-132. Menlo Park, CA: Addison-Wesley.

Swift, C. O. and L. Denton. 2003. Cross-cultural experiential simulation in the global marketing classroom: Bafa-Bafa and its variants. Marketing Education Review, 13 (3): 41.

Tajfel, H. and J. C. Turner. 1985. The social identity theory of intergroup behavior. In Worchel, S. W. and W. G. Austin (Eds), Psychology of intergroup relations. 2nd ed: 7-24. Chicago: Nelson-Hall.

Tashakkori, A. and C. Teddlie (Eds). 2003. Handbook of mixed methods in social and behavioral research. Thousand Oaks, CA: Sage Publications.

Taylor, E. B. 1871. Primitive culture. London: JohnMurray.

Teddlie, C. and A. Tashakkori. 2003. Major issues and controversies in the use of mixed methods in the social and behavioral sciences. In Tashakkori, A. and C. Teddlie (Eds), Handbook of mixed methods in social and behavioral research: 3-50. Thousand Oaks, CA: Sage Publications.

Thomas, D. C. 2006. Domain and development of cultural intelligence: The importance of mindfulness. Group Organization Management, 31 (1): 78-99.

Thomas, D. C., E. Elron, G. Stahl, B. Z. Ekelund, E. C. Ravlin, J.-L. Cerdin, S. Poelmans, R. Brislin, A. Pekerti, Z. Aycan, M. Maznevski, K. Au, and M. B. Lazarova. 2008. Cultural intelligence: Domain and assessment. International Journal of Cross Cultural Management, 8(2): 123-143.

Thomas, D. C. and K. Inkson. 2003. Cultural intelligence: People skills for global business. San Francisco: Berrett-Koehler Publishers, Inc.

Thomas, D. C., G. Stahl, E. C. Ravlin, S. Poelmans, A. Pekerti, M. Maznevski, M. B. Lazarova, E. Elron, B. Z. Ekelund, J.-L. Cerdin, R. Brislin, Z. Aycan, and K. Au. 2008. Development of the cultural intelligent assessment. Paper presented at The Academy of International Business (AlB) Annual Meeting 2008. Milan.

Thomas, G. and D. James. 2006. Reinventing grounded theory: Some questions about theory, ground and discovery. British Education Research Journal, 32 (6): 767-795.

Thorndike, E. L. 1920. Intelligence and its use. Harper's Magazine, 140: 227-235.

Thorne, M. L. 2000. Cultural chameleons. British Journal of Management, 11 (4): 325-339.

Tina, K. 2004. Commentary: Expert researchers and audit trails. Journal of Advanced Nursing, 45 (2): $134-$ 135.

Tobin, G. A. and C. M. Begley. 2004. Methodological rigour within a qualitative framework. Journal of Advanced Nursing, 48 (4): 388-396.

Tong, A., P. Sainsbury, and J. Craig. 2007. Consolidated criteria for reporting qualitative research (COREQ): A 32-item checklist for interviews and focus groups. International Journal for Quality in Health Care, 19 (6): 349-357. 
Toyne, B. 1989. International exchange: A foundation for theory building in international business. Journal of International Business Studies, 20 (1): 1-17.

Toyne, B. and D. Nigh. 1998. A more expansive view of international business. Journal of International Business Studies, 29 (4): 863-875.

Triandis, H. C. 1972. The analysis of subjective culture. London: Wiley-Interscience.

Triandis, H. C. 1994. Culture and social behavior. New York: McGraw-Hill.

Triandis, H. C. 2001. The study of cross cultural management and organization: The future. International Journal of Cross Cultural Management, 1 (1): 17-20.

Triandis, H. C. 2006. Cultural intelligence in organizations. Group Organization Management, 31 (1): 20-26.

Tripp, D. H. 1985. Case study generalisation: An agenda for action. British Educational Research Journal, 11 (1): $33-43$.

Trompenaars, F. and C. Hampden-Turner. 1998. Riding the waves of culture: Understanding diversity in global business. 2nd ed. New York: McGraw-Hill.

Tsoukas, H. 1991. The missing link: A transformational view of metaphors in organizational science. Academy of Management Review, 16 (3): 566-585.

Turner, B. A. 1983. The use of grounded theory for the qualitative analysis of organizational behaviour. Journal of Management Studies, 20 (3): 333-348.

Ulijn, J. and K. St Amant. 2000. Mutual intercultural perception: How does it affect technical communication? - some data from China, the Netherlands, Germany, France and Italy. Technical Communication, 47 (2): 220-237.

Urquhart, C. 2002. Regrounding grounded theory or reinforcing old prejudices? A brief reply to Bryant. Journal of Information Technology Theory and Application, 4 (3): 43-55.

Usunier, J.-C. 1998. International and cross-cultural management research. London: Sage Publications.

Vaughan, D. 1992. Theory elaboration: The heuristics of case analysis. In Ragin, C. and H. S. Becker (Eds), What is a case? Exploring the foundations of social inquiry: 173-202. New York and Cambridge: Cambridge University Press.

Verkerk, M. J. 2004. Trust and power on the shop floor. Delft: Eburon.

Verschuren, P. 2003. Case study as a research strategy: Some ambiguities and opportunities. International Journal of Social Research Methodology, 6 (2): 121-139.

Vossestein, J. 2004. Dealing with the Dutch: The cultural context of business and work in the Netherlands. 14th (revised) ed. Amsterdam: KIT Publishers.

Wagner, R., K. 2000. Practical intelligence. In Sternberg, R. J., editor, Handbook of intelligence: Theories, measurements, and applications: 380-395. Cambridge: Cambridge University Press.

Walker, D. and F. Myrick. 2006. Grounded theory: An exploration of process and procedure. Qualitative Health Research, 16 (4): 547-559.

Wall, S. 2006. An autoethnography on learning about autoethnography. International Journal of Qualitative Methods, 5 (2): 146-160.

Warner, M. 2004. Introduction: Culture and management in Asia. In Warner, M., editor, Culture and management in Asia: 1-23. London: Routledge.

Weick, K. E. 1989. Theory construction as disciplined imagination. Academy of Management Review, 14 (4): 516-531.

Weick, K. E. 1995. What theory is not, theorizing is. Administrative Science Quarterly, 40: 385-390.

Welzel, C. 2007. A human development view on value change. http://www.worldvaluessurvey.org/. Accessed September 2008.

Wenger, E. C. 1998. Communities of practice: Learning, meaning, and identity. New York: Cambridge University Press.

Wenger, E. C. and W. M. Snyder. 2000. Communities of practice: The organizational frontier. Harvard Business Review, 78 (1): 139-145.

White, C. and L. Boucke. 2006. The UnDutchables: An observation of the Netherlands, its culture and its inhabitants. 5th ed. Lafayette, CO: White-Boucke Publishing Inc.

Whittemore, R., S. K. Chase, and C. L. Mandle. 2001. Validity in qualitative research. Qualitative Health Research, 11 (4): 522-537.

Winter, G. 2000. A comparative discussion of the notion of 'validity' in qualitative and quantitative research. The Qualitative Report, 4 (3 \& 4). http://www.nova.edu/ssss/QR/QR4-3/winter.html. Accessed March 2009.

Wolcott, H. F. 2002. Writing up qualitative research ... better. Qualitative Health Research, 12 (1): 91-103.

Wolman, B. B. (Ed). 1985. Handbook of intelligence: Theories, measurements, and applications. New York: John Wiley \& Sons. 
Woodside, A. G. and E. J. Wilson. 2003. Case study research methods for theory building. Journal of Business and Industrial Marketing, 18 (6/7): 493-508.

Worchel, S. W., F. Morales, D. Paez, and J.-C. Deschamps (Eds). 1998. Social identity: International perspectives. London: Sage Publications.

Wright, L. L. 2004. The need for international qualitative research. In Punnett, B. J. and O. Shenkar (Eds), Handbook for international management research. 2nd ed: 49-67. Ann Arbor: The University of Michigan Press.

Wright, R. W. 1970. Trends in international business research. Journal of International Business Studies, 1 (1): 109-123.

Wright, R. W. and D. A. Ricks. 1994. Trends in international business research: Twenty-five years later. Journal of International Business Studies, 25 (4): 687-701.

Yamazaki, Y. and D. C. Kayes. 2004. An experiential approach to cross-cultural learning: A review and integration of competencies for successful expatriate adaptation. Academy of Management Learning and Education, 3 (4): 362-379.

Yardley, L. 2000. Dilemmas in qualitative health research. Psychology and Health, 15 (2): 215 - 228.

Yeganeh, H., Z. Su, and E. V. M. Chrysostome. 2004. A critical review of epistemological and methodological issues in cross-cultural research. Journal of Comparative International Management, 7 (2): 66-86.

Yin, R. K. 1981a. The case study as a serious research strategy. Science Communication, 3 (1): 97-114.

Yin, R. K. 1981b. The case study crisis: Some answers. Administrative Science Quarterly, 26 (1): 58-65.

Yin, R. K. 2008. Case study research: Design and methods. 4th ed. Thousand Oaks, CA: Sage Publications.

Zhu, Y. and J. Ulijn. 2005. Introductory essay: New horizons in cross cultural management. Cross Cultural Management, 12 (3): 4-13.

Znaniecki, F. 1934. The method of sociology. New York: Farrar \& Rinehart. 


\section{NederLandse SAMENVATting}

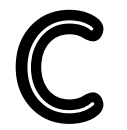

ROSS-CULTURELE VRAAGSTUKKEN STAAN IN HET BRANDPUNT VAN DE BELANGSTELLING. Opmerkelijk daarbij is dat zowel in het publieke debat als in de wetenschappelijke discussie de aandacht vooral - zo niet uitsluitend - uitgaat naar botsingen tussen verschillende culturen. Dat geldt al sinds het pionierswerk van cultuuronderzoekers Hofstede (Culture's Consequence 1980) en Huntington (The Clash of Civilizations? 1999). Natuurlijk, culturen verschillen van elkaar en de uiterste consequentie van die verschillen is dat de interactie tussen die verschillende culturen tot conflicten leiden en op die manier kan de enorme aandacht voor dit verschijnsel ook worden gerechtvaardigd. Dat doet echter niets af aan de ironie van het feit, dat terwijl het streven naar constructieve samenwerking ten grondslag ligt aan het onderzoek naar de wisselwerking tussen culturen, de meeste aandacht wordt geschonken aan de destructieve kant van het proces. We nemen graag aan dat het verbeterde inzicht in conflicterende culturen ons helpt om effectiever met de gevolgen ervan om te gaan. De vraag dient zich echter aan of het geen tijd is om ons perspectief op cross-culturele interactie te verbreden en ons niet alleen meer te blijven focussen op de conflictkant. Zo'n omvattender visie zal niet alleen ons begrip vergroten, en rijkere alternatieven bieden aan het onderzoek, maar ons tevens leren effectiever te opereren in de praktijk van de cross-culturele wisselwerking.

Deze studie gebruikt gegevens uit kwalitatief veldonderzoek voor de opbouw van een theorie over cultuur en leerprocessen in organisaties. Die gegevens werden verzameld door eigen observaties en diepte-interviews met Nederlandse en Thaise managers en werknemers in dienst bij vijf Nederlandse firma's in Thailand. De studie ving aan in januari 2005 en omvatte twee hoofdfasen. Allereerst de verkennende open gesprekken (lente van 2005) met vijf Nederlandse managers met een ruime ervaring in de samenwerking met Thaise en Aziatische collega's, klanten en ambtenaren. Deze verkenning heeft richting gegeven aan het veldonderzoek in de tweede fase (februari en maart 2006). Dit veldonderzoek richtte zich op vijf Nederlandse firma's in Thailand. In deze bedrijven werden 21 diepgaande gesprekken gevoerd over cross-culturele samenwerking van Europese en Aziatische managers en medewerkers op sleutelposities in het bedrijf. Bovendien deed de mogelijkheid zich voor die wisselwerking in de praktijk te observeren. In deze fase werden bovendien een drietal (praktijk)deskundigen met grote ervaring en inzicht in cross-cultureel management en de wisselwerking tussen Nederlandse/Westerse en Thaise/Aziatische culturen geraadpleegd. Ten slotte werd ook gebruik gemaakt van informele gesprekken met talrijke 'toevallige informanten' (of: accidental informants).

In methodologische zin vormt het stramien van deze studie een combinatie van Grounded Theory en case study research. 'Grounded Theory' is een opkomende 
inductieve theorie-vormende methodologie die voortkomt uit het pionierswerk van Glaser en Strauss (1967). Deze benadering wint aan terrein, omdat zij een nieuwe impuls geeft aan de wijze waarop binnen de sociale wetenschappen theorieën worden ontwikkeld. Empirisch materiaal wordt in deze benadering ingezet om langs een gestructureerde, maar ook flexibele weg tot de ontwikkeling van theoretische begrippen en hun onderlinge relaties te komen. Dat vraagt een voortdurende iteratie tussen de waarnemingen en de 'theorie in wording'. Deze methodologie is bijzonder geschikt voor kwalitatief onderzoek naar moeilijk af te bakenen sociale processen (Suddaby 2006). Naast de gegevens uit het veldonderzoek werd ook een uitvoerige literatuurstudie verricht naar begrippen en hun onderlinge verbanden met betrekking tot cross-culturele verschillen en -verhoudingen. Beide studies vormden de input voor de theorieopbouw. In deze benadering beschouwt men ook literatuur als evidence, met in het achterhoofd de gedachte dat in de praktijk een onderzoeker nooit in staat is om geheel onbevangen, met een schone lei de empirische werkelijkheid te interpreteren. Bij de analyze en rapportage werd gebruik gemaakt van het stramien van de case-analysis. Deze benadering brengt structuur in de data en maakt het empirische materiaal toegankelijk voor de lezer (Hoofdstuk 2).

Uiteindelijk leverde deze inspanning drie uitgebreide beschrijvende 'cases' en twee 'vignettes' (beschrijvende 'portretten' of 'karakterschetsen') op. Deze beschrijvingen vormen uiteindelijk 'de theoretische memoranda', een noodzakelijk analytisch instrument binnen 'Grounded Theory'. Het resultaat van de case analysis, de Grounded Theory, en de literatuurstudie (of: theoretical sampling) is tenslotte een 'Grounded Theory' van: cross-culturele intelligentie (XCQ) temidden van verwikkelde culturele webben, waarin twee theoretische kernconcepten zijn te onderkennen: verwikkelde culturele webben (of: intricate cultural web) en cross-culturele intelligentie (Hoofdstuk 4 en 6). Deze theorie ontvouwt vier belangrijke vernieuwende perspectieven op cultuur en leerprocessen in organisaties.

Ten eerste, bepleit de theorie een holistische visie op cultuur, in tegenstelling tot het meer gangbare en veelal gepopulariseerde dimensionale perspectief. In de gepresenteerde visie wordt cultuur beschouwd als een gedeelde wijze van zijn en betekenisgeving ('being and sense-making') die uniek is voor een bepaalde groep mensen en die de groep onderscheidt van haar tegenhangers. De leden van een cultuur zijn te beschouwen als 'culturele eenheden' en deze eenheden functioneren op talrijke cross-culturele platformen. Het eerste kernconcept, verwikkelde culturele webben is een metafoor, waarmee uitdrukking wordt gegeven aan de haast onvoorstelbare complexiteit van culturele wisselwerkingen. De metafoor vloeit voort uit de constatering, dat culturele eenheden, of dat nu individuen, groepen of organisaties zijn, niet slechts functioneren in enkelvoudige sociale structuren en netwerken, maar deel uitmaken van een veelheid van sociale verbanden, die steeds weer andere normen en waarden aanleggen. Die verbanden, netwerken, of 'webben' zijn met elkaar vervlochten. Men kan daarbij denken aan nationale, etnische en politieke verbanden en verbanden die bijvoorbeeld te maken hebben met levensbeschouwing, 
geslacht, beroepsgroep, werkkring, of specifieke organisaties. De verwikkelde webben drukken zowel hun stempel op de culturele eenheid, als op de wisselwerking tussen culturele eenheden en de context (of: cultureel platform) waar zij elkaar ontmoeten, en vormen daarmee een fundamentele eigenschap van culturele eenheden. Met andere woorden: verschillende culturele webben werken tegelijkertijd in op het gedrag van die culturele eenheden. De cross-culturele interface heeft betrekking op de wisselwerking tussen culturele eenheden uit diverse culturele identiteiten. Die wisselwerking vormt een sociaal proces in de context (of: cross-cultural platform) waarin twee of meer culturele entiteiten elkaar raken. Op die manier hebben verwikkelde culturele webben een grote invloed op cross-culturele processen. In deze visie op cultuur zijn alle individuen te beschouwen als virtuele multiculturele eenheden en brengen alle sociale processen onveranderlijk cross-culturele wisselwerking met zich mee.

Ten tweede, de voorgestelde theorie verbreedt ons perspectief op de crossculturele wisselwerking. Dat perspectief reikt verder dan het conflict en de botsing (of: conflict modus), die in de gangbare literatuur zo uitvoerig aan de orde komen. Uitgangspunt is dat er meer soorten wisselwerking bestaan dan het conflict. Het begrip 'cross-cultureel' heeft in deze theorie niet alleen betrekking op de vergelijking van de kenmerken van twee of meer culturen, zoals dat in het traditionele onderzoek, maar richt zich met name op het facet wisselwerking (of: interactie) in cross-culturele verschijnselen die wij willen doorgronden. Deze leidende gedachte heeft samen met de verzamelde data geresulteerd in een uitgebreide taxonomie van cross-culturele wisselwerking (of: cross-cultural condition) (Hoofdstuk 6). Het is niet verwonderlijk dat het conflict een van de wisselwerkingsvormen is, maar daarnaast vinden we in deze taxonomie drie andere vormen van wisselwerking. De vier wisselwerkingsvormen in deze taxonomie zijn:

- Conflict. Verschillen worden op de spits gedreven; het gaat vooral om beheersing;

- Wederkerig belang. Er bestaat tussen de actoren een zekere mate van wederzijds belang en bereidheid tot 'geven en nemen'. Uitwisseling is het sleutelwoord;

- Unificatie. Er bestaat affiniteit tussen de actoren en actoren nemen elementen van de cultuur van de ander over. Er is een tendends om culturen te kruisen (hybridizing);

- Variatie. Hier is empathie het sleutelwoord. Actoren kruipen in de huid van de ander en passen zich aan aan de culturele omgeving.

In de voorgestelde theorie omvatten de vier interactiewijzen het hele scala van mogelijkheden. Deze interactiewijzen sluiten elkaar niet uit en kunnen naast elkaar bestaan en doen dat feitelijk ook vaak, al kan in een bepaalde cross-culturele context de ene vorm veel dominanter zijn dan de andere. De vier interactiewijzen vormen samen een dynamisch, non-lineair pad dat voor altijd voortgaat. Deze taxonomie en de dynamiek waaruit die is voortgekomen, brengt ons er toe de fixatie op het conflict als 
interactievorm op te geven, en ons open te stellen voor andere, meer subtiele en toch vitale vormen van cross-culturele interactie.

Ten derde, de voorgestelde theorie weerhoudt ons er van de gangbare weg te kiezen van het analytisch reductionisme, waarin de wisselwerking wordt afgebroken in atomistische eenheden. De theorie zet ons er wel toe aan ons vooral te richten op het proces van cross-culturele wisselwerking. Deze holistische en kwalitatieve kijk op cross-culturele interactie als een dynamisch sociaal proces impliceert twee onderling nauw samenhangende aspecten: actie en zijn context. De theorie probeert een antwoord te vinden op de intrigerende vraag hoe sociale actoren erin slagen om in de grote complexiteit van verwikkelde culturele webben effectief te functioneren. De theorie suggereert dat sociale actoren in het dagelijks bestaan een praktische 'heuristieke' bekwaamheid aanleren die hen in staat stelt in steeds weer andere situaties een effectieve interactie aan te gaan met vertegenwoordigers van andere culturen.

Deze heuristieke bekwaamheid vormt het tweede kernbegrip van de voorgestelde 'Grounded Theory': cross-culturele intelligentie, of XCQ (Hoofdstuk 6). XCQ, zo genoemd om het te onderscheiden van het meer algemene begrip 'culturele intelligentie' of $\mathrm{CQ}$, een recent onderzoekthema waarvan gelijktijdig door twee prominente onderzoeksteams de weg wordt bereid - Ang en haar collega's (Ang en Van Dyne 2008, Earley en Ang 2003) en Thomas en zijn team (Thomas 2006, Thomas, Elron, Stahl, Ekelund, Ravlin, Cerdin, Poelmans, Brislin, Pekerti, Aycan, Maznevski, Au, en Lazarova 2008). Het concept XCQ vormt een uitbreiding van het concept $C Q$ en is vooral bedoeld om recht te doen aan de bekwaamheden van actoren effectief te functioneren in situaties waarin culturen direct met elkaar in wisselwerking staan. XCQ vormt het vermogen van culturele eenheden om cross-culturele interactie met elkaar te hebben met het doel om gewenste en wenselijke resultaten tot stand te brengen. Daarmee is XCQ het in de praktijk aangeleerde vermogen om een cross-culturele heuristiek effectief toe te passen ('learning by doing'). Het concept XCQ zoals die in deze studie wordt geïntroduceerd kan op die manier gezien worden als een aanvulling op de onderzoeksinspanning rond $\mathrm{CQ}$. Het begrip XCQ is in die zin door zijn holistische en kwalitatieve karakter complementair aan de snel expanderende kwantitatieve inspanningen ( Hoofdstuk 6).

Tenslotte, biedt deze studie niet alleen een nieuw raamwerk voor onderzoek naar cross-culturele wisselwerking, maar ook voor de ontwikkeling van XCQ bij individuen en groepen. Centraal daarin is het begrip "heuristiek" (Hoofdstuk 6). De term "heuristiek" wordt frequent in de informatica gebruikt voor het oplossen van zeer complexe problemen met behulp van relatief eenvoudige routines. Heuristieken worden toegepast als de complexiteit en dynamiek van een probleem of situatie zo hoog is, dat er geen sluitende algoritmen kunnen worden ontwikkeld. Met de heuristiek neemt men dan genoegen met relatief simpele oplossingen, die weliswaar niet volmaakt zijn, maar waarvan bewezen is dat ze 'werken'. De theorie veronderstelt dat sociale actoren die opereren in de complexiteit en dynamiek van de 'verwikkelde 
culturele webben' in de loop van de tijd in de praktijk dergelijke heuristieken aanleren. De theorie onderscheidt vier stappen in dit ontwikkelingsproces. 1) Het bewust worden en opruimen van eigen vooroordelen en het uitstellen van oordelen ('negative capacity'); 2) het aftasten van de situatie om te begrijpen hoe de context in elkaar zit en welke eisen daarin aan actoren gesteld worden; 3) het aanleggen van situationele verankeringen: vaststellen wat de actor wil bereiken en het vinden van wegen die zich in de betreffende situatie daarvoor eigenen; en 4) het toepassen van cross-culturele mechanismen en instrumenten om de gewenste situatie te realiseren. Met de toepassing van heuristiek raken we het grondprincipe van deze studie: het principe van 'XCQ-in actie'. De belangrijkste boodschap voor kennisontwikkeling over crossculturele wisselwerking is, dat we moeten leren van de wijzen waarop mensen (culturele eenheden) in de praktijk oplossingen vinden voor problemen die door die wisselwerking worden opgeroepen. Het gaat om het feitelijke, waarneembare gedrag waarmee mensen in die wisselwerking hun doelstellingen effectief verwezenlijken: $X C Q$-in actie.

Zoals voor elk onderzoek, gelden voor deze studie een aantal beperkingen. Op de eerste plaats moeten we vaststellen dat de methodologische keuzes die voor deze studie werden gemaakt juist door de voordelen die zij boden, ook nieuwe methodologische uitdagingen introduceerden. Methodologisch gezien vraagt de interpretatieve 'Grounded Theory' benadering zowel dat de onderzoeker als zijn publiek voortdurend op hun hoede zijn voor impliciete interpretaties van waarnemingen. Onderzoeksresultaten dienen te worden beschouwd als informed interpretation, aangeleverd door een bepaalde interpretator, met een eigen specifiek profiel. Daarnaast is binnen de relatief jonge traditie van 'Grounded Theory' de mogelijkheid om voort te bouwen op eerdere ervaringen beperkt. Bovendien vormt, theoretisch gezien, het thema van culturele intelligentie een opkomende nieuwe onderzoekstroom en dragen de meeste studies over dit onderwerp in dit stadium een verkennend karakter. Tenslotte moeten we ons er van bewust zijn dat deze studie is gebaseerd op waarnemingen op één enkel cross-cultureel podium van de Nederlandse en Thaise actoren in vijf bedrijven. Het is duidelijk dat een grotere verscheidenheid aan situaties meer en beter bewijsmateriaal zou kunnen opleveren en ons in staat zou stellen het model verder te valideren en te verrijken. Bewust van deze beperkingen, is ernaar gestreefd de beschikbare validatiemethoden en -procedures die specifiek voor kwalitatief-interpretatief onderzoek worden aanbevolen, waar mogelijk toe te passen (Hoofdstuk 2). Dit laat onverlet, dat wanneer het project met meer middelen (onderzoekers, tijd en financiën) zou zijn gezegend, de validatie een robuster karakter had kunnen krijgen. Dit is een cruciale les voor toekomstig onderzoek (Hoofdstuk 8).

Terwijl de onderzoekster zich bewust is van de beperkingen van deze studie is zij ervan overtuigd dat de verkregen inzichten nieuwe wegen openen, niet alleen met betrekking tot onderzoek naar cross-culturele interactie, maar ook voor het ontwikkelen van cross-culturele intelligentie in de praktijk. De studie draagt twee belangrijke onderzoeksthema's aan, die dringend tot verdere analyze uitnodigen. Het 
eerste thema betreft het cultiveren van XCQ. Ook hier moet de nadruk liggen op het in de praktijk brengen van XCQ: 'XCQ-in actie' en daarmee gaan we verder dan een louter cognitieve, emotionele, of gedragsmatige aanpak. In plaats van naar deelaspecten te kijken, richten we ons in dit onderzoek vooral op de actie in de praktijk waarin die aspecten juist geïntegreerd zijn. Hierbij valt een parallel te trekken met de ontwikkeling van andere stilzwijgende ('tacit') vaardigheden, waarbij praktijk en begeleiding (of: coaching) direct zijn gekoppeld. In dat perspectief mogen wij van conventionele XCQ-cultiveringsprogramma's binnen de beslotenheid van het klaslokaal niet te veel verwachten. Het gaat er om onderzoek te doen en programma's in de praktijk te ontwikkelen. Alleen dan kan een proces op gang gebracht worden waarin individuen en groepen ageren en reageren op de situatie waarin zij zich bevinden en op die manier leren van de opgedane ervaringen. Trainers en coaches kunnen in dat proces een belangrijke faciliterende rol spelen.

Het tweede onderzoekthema betreft het vraagstuk van de beoordeling van CQ en $X C Q$. Tot nu toe wordt het onderzoek naar culturele intelligentie of $C Q$ vooral gedreven door de ambitie om harde kwantitatieve criteria te ontwikkelen. De parallel met het onderzoek op het gebied van de algemene intelligentie, en het resulterende intelligentie-quotient (IQ) ligt daarbij voor de hand. Deze onderzoeksbenadering mag zeer vruchtbaar en nuttig zijn, maar men kan niet voorbij gaan aan de beperkingen die kleven aan het zeker tot de verbeelding sprekende model van een IQ-test. Het belangrijkste bezwaar van dit model is de radicale loskoppeling van de realistische context en de daadwerkelijke actie. Het tweede bezwaar is de onvermijdelijke vertekening (of: bias) die inherent is aan elke psychologische test, die gebaseerd is op zelf-rapportering.

De tijd dringt om de 'CQ zwarte doos' open te wrikken en geen genoegen meer te nemen met de beperkingen van de kwantitatieve beoordeling van CQ en daarmee de grens van CQ-onderzoek te overschreiden. Moge nogmaals duidelijk zijn dat de begrippen $C Q$ en XCQ, zoals die in deze studie zijn toegelicht, samen de sleutel zouden kunnen vormen tot de geheimzinnige cross-culturele zwarte doos. Samen bieden deze twee onderzoeksthema's ruime kansen voor toekomstige onderzoeksprogramma's waarmee ons begrip van cross-culturele fenomenen kan worden vergroot. Daarmee kan een constructieve bijdrage worden geleverd aan de samenwerking tussen verschillende culturen binnen en tussen organisaties (Hoofdstuk 8).

\footnotetext{
D EZE STUDIE HEEFT EEN AANTAL INZICHTEN IN CROSS-CULTURELE WISSELWERKING AAN HET LICHT gebracht en zaken die daarmee nauw in verband staan. Het betreft inzichten die ofwel te weinig zijn onderzocht ofwel zijn ontsnapt aan de aandacht van onderzoekers. Deze inzichten nodigen uit om nieuwe richtingen in te slaan, niet alleen in de huidige praktijk maar ook in toekomstig onderzoek naar de essentie van cultuur en leerprocessen in organisaties.
} 


\section{SUMMARY IN ENGLISH}

區

Ver since the landMark Publication of Hofstede's Culture's Consequences (Hofstede 1980) and Huntington's The Clash of Civilizations? (Huntington 1993), much of the world's and academic attention on cross-cultural matters has concentrated heavily, if not exclusively, on the clashing of cultural differences. Indeed culture differs, and clashing is the most dramatic consequence of cross-cultural interaction, thereby justifying the enormous attention paid to the phenomenon. Thus, despite the quest for peaceful and constructive co-operation that underlies crosscultural investigation, we have ironically paid most of our attention to the one potentially damaging aspect of the process. No doubt, greater insights into the clashing effect of cultures have helped us deal better with its consequences. Yet, perhaps the time has come to expand our view of cross-cultural interactions beyond the clashing facet. Such an expanded view will not only further strengthen our understanding but also better equip us with richer alternatives in exploring and exploiting cross-cultural interfaces. Indeed, based on this study's evidence and its eventual findings, a fresh new view of culture and its consequences emerged that import new opportunities to advance our collective effort in cross-cultural issues further.

This study built a grounded theory of culture and learning in organizations from evidence collected through observation of and open-ended interviews with Dutch and Thai employees working for five selected Dutch firms in Thailand. The study commenced in January 2005 and involved two major phases of evidence collection and analysis. First, interviews conducted in the spring of 2005 with five Dutch executives who had extensive experience working with Thai and Asian colleagues, customers, and officials - provided initial evidence that shaped the sampling, content, and direction of the field study conducted in Thailand during the period February and March 2006. This field study included visits to four Dutch firms in Thailand for extended interviews with and field observations of a selected number of their Dutch and Thai employees who interacted on a regular basis, resulting in 21 intensive open-ended interviews on their cross-cultural experiences. This phase also included consultation with three experts in both cross-cultural management and the interplay between Dutch/Western and Thai/Asian cultures. Further supplementary evidence was gathered through informal conversations with numerous 'accidental informants'.

All evidence was collected and analyzed within the procedural mandate of grounded theory in combination with case study research. Grounded theory, an emerging inductive theory building methodology pioneered by Glaser and Strauss (1967), has gained much attention in recent years due to its fresh approach to theorizing, namely through flexible yet structured inductive analytical procedures that focus on letting empirical evidence from the field reveals theoretical constructs and their relation through the use of constant-comparison and specific coding procedures. It is a 
theory building methodology particularly well-suited for qualitative inquiry of social process with fuzzy nature (Suddaby 2006). Grounded evidence for this study came from two sources - first from the field investigation described above, and second from review of selected literature related to the concepts and constructs as they emerged. Treating literature as evidence helped solve the tension between the ideality of the demand that a grounded theorist begin with a clean slate and the reality of unavoidable intellectual imprint. Case study was subsequently employed as a complementary analytical and reporting instrument in the fashion suggested by seasoned scholars (Chapter 2).

Eventually, the theorizing effort yielded three thick descriptive cases (Chapter 3, 5 and 7) and two vignettes (Chapter 8) that serve as the ultimate 'theoretical memos' - a mandated theorizing instrument in grounded theory. Through case analysis of grounded evidence and theoretical sampling of pertinent literature, the key finding of this study emerged as a grounded theory of cultural intelligence by the name of crosscultural intelligence (XCQ) amid intricate cultural webs, that comprises two core theoretical concepts: intricate cultural webs and cross-cultural intelligence (Chapter 4 and 6). The proposed theory unveils four major fresh perspectives on culture and learning in organizations.

First, the theory advocates a holistic view of culture, as opposed to the more popular and much popularized dimensional view. Under this view culture is conceptualized as a shared way of being and sense-making that is unique to a distinct group of people and can distinguish that group from their counterparts. Members of a culture are cultural units and these units must operate in numerous cross-cultural platforms. The first core theoretical concept of intricate cultural webs then denotes the property that characterizes first cultural unit (an entity), second cross-cultural interface (a process), and third, cross-cultural platform (a condition or context) in which this process transpires. Intricate cultural webs denote the fundamental property of any cultural unit because all cultural units (even single individuals) invariably belong to or are made up of more than one cultures (national, organizational, professional, generational, gender, ethnic, and myriad other cultural categories) and their property can be metaphorically depicted as intricate cultural webs, whose identity is made up of diverse cultural traits that simultaneously influence the operation of those cultural units. Interaction between cultural units of diverse cultural identities denotes cross-cultural interface, a social process that transpires in a cross-cultural platform or a social setting that entails two or more competing cultures. Again, intricate cultural webs characterize crosscultural condition and the social process that transpires within that context. In essence, under this view of culture, all individuals are virtually multi-cultural units and all social processes invariably involve cross-cultural interaction.

Second, the proposed theory substantially expands a view of what possible modes a cross-cultural condition can manifest - a view that extends far beyond the aforementioned well-documented and much popularized clashing mode. The term 'crosscultural' as used and meant in this study has a specific meaning and serves an impera- 
tive choice in the proposed theory. In brief, the term 'cross-cultural' here signifies not only the traditional comparative treatment of two or more cultures, but also, and even more importantly, the interaction facet of a cross-cultural phenomenon. Given such imperative and based on this study's evidence, an expanded taxonomy of crosscultural condition emerged (Chapter 6 ). The theorized taxonomy naturally includes the clashing mode but also extends far beyond that to altogether encompass four distinct modes of cultural crossing: clashing, reciprocal, unification and variation. The four modes, while distinct from each other in their characterization and implication, are found to be collectively exhaustive, yet not mutually exclusive. These modes can and do co-exist, although one may be more dominant than others in a particular crosscultural context. The four modes maintain an inter-related dynamic path that is forever proceeding, yet not linearly progressing. The theorized taxonomy and its dynamics reveal several insights that may have escaped investigation in the past and compels us to move beyond our fixation on the clashing consequence and extend our attention to other, more subtle yet as vital, possible consequences of cross-cultural life.

Third, the proposed theory instigates an alternate look at cross-cultural interaction that puts sole emphasis on its very nature as a process, instead of the standard compartmentalized treatment that deconstructs the process into atomistic units. This holistic and qualitative view of cross-cultural interaction as a dynamic social process entails two interrelated aspects: action and its context. To be precise, the proposed theory conceives the heart of cross-cultural process to be a heuristic based strategic capability that enables social actors to operate successfully in the midst of the complex cultural force that is characterized by the complexity of intricate cultural webs. This heuristic based capability constitutes the second core theoretical concept of the proposed grounded theory: cross-cultural intelligence, or XCQ (Chapter 6). XCQ, so named to differentiate it from the more general 'cultural intelligence' or $C Q$, an emerging research theme pioneered concurrently by two prominent research groups - Ang and her colleagues (Ang and Van Dyne 2008, Earley and Ang 2003) and Thomas and his team (Thomas 2006, Thomas et. al. 2008). Specifically, the concept of XCQ is an expansion of the CQ concept to stress its import not simply when cultural units operate in unfamiliar cultural contexts but in situations in which cultures specifically cross. Thus, $X C Q$ constitutes the facility or power to exercise cross-cultural interaction to achieve desired and desirable results when cultural units operate in a cross-cultural interface. That is, XCQ is a learning-by-doing execution of the ability to practice cross-cultural heuristic effectively. The XCQ concept as theorized in this study offers an additional angle to the entire effort on the CQ research stream primarily because of its holistic and qualitative nature that complements well the burgeoning atomistic and quantitative effort currently pursued by the two major research groups on CQ (Chapter 6).

Finally, this study offers a new framework for an examination of cross-cultural interaction through the concept of a simple heuristic procedure which lies at the heart of XCQ (Chapter 6). This simple heuristic procedure calls for four interlinked, goaloriented deliberations: 1 ) invoking negative capability to achieve an open-mind; 2) 
surveying the situation to gain an understanding of the contextual attributes and demands; 3) setting situational anchorage against desired and desirable benchmark; and 4) applying cross-cultural interaction mechanisms that are compatible with the context and the identified goal. The exercise of this heuristic accentuates the fundamental tenet of this study's findings - the tenet of 'XCQ in action'. XCQ in action encapsulates the study's key message that the road ahead for future application and investigation must be anchored in looking at XCQ in action that is framed within the theorized XCQ heuristic procedure.

As with all research, this study was constrained by a number of limitations. First, the strategic choices made for this study entails certain challenges, despite, or actually because of, the benefits they offered. Methodologically, the interpretive grounded theory approach calls for acute awareness of the burden of interpretation, both on the researchers and their audience. That is, study results must be carefully taken as an 'informed' interpretation made by a particular interpreter with a specific profile. Also, because grounded theory is a relatively young methodology, the opportunity to learn from previous experience is somewhat limited. Next, theoretically, the theme of cultural intelligence, as earlier mentioned, is an emerging research stream and most studies on this topic will turn out to be exploratory in nature at this early stage. Finally, the study and its findings were based on one single cross-cultural platform of the Dutch and Thai actors in the five studied firms. Obviously, greater variety of such platform would yield richer evidence to further validate and enrich the model. To address these limitations, all appropriate validation methods and procedures recommended specifically for qualitative-interpretive inquiry such as this were applied and proved to have been most indispensible (Chapter 2). Without them, there might have been more and greater limitations. Yet, no doubt, if the project were to have been blessed with more resources (investigator, time and finance), the study could have benefited even further from a more robustly employed validation effort. This is a crucial lesson for future research that suggests many implications (Chapter 8).

Eventually, despite its limitations, this study paves new ways to future effort in cross-cultural issues, for both practitioners and researchers. In brief, this study instigates two main research themes that are in pressing need of deeper and broader investigations. The first theme relates to the issue of cultivation. Given the imperative principle of XCQ in action, research into the cultivation of XCQ must take on a turn that goes beyond cognitive, psychological, or behavioural orientation, but seek to fuse these together via action. Hence, cultivation of XCQ should take a similar approach as that commonly used in development of other tacit skills - an approach that couples practicing with coaching. Therefore, instead of looking into XCQ cultivation programs that follow conventional classroom orientation, future research should seek to develop programs that put cultural units at the heart of a real, or realistically simulated, setting and let them act and react to the situation and learn from those actual experiences, with trainers acting as coaches, not instructors. The second research theme concerns the matter of the assessment of $\mathrm{CQ}$ and $\mathrm{XCQ}$. Assessment of cultural intelligence or 
$\mathrm{CQ}$, particularly in rigorous quantitative manner, has been the main motivating force and central effort in the research on cultural intelligence to date - hence the inherited abbreviated term $\mathrm{CQ}$, which heavily resonates with the popular notion of IQ. Immensely prolific and useful as this stream of research is, it also inherits the limitations commonly associated with the inspiring model of an IQ test, the most important of which are first a detachment from real context and actual action and second the inevitable bias inherent in the self-reporting approach extensively used in such tests. There is thus a pressing need to pry open the ' $\mathrm{CQ}$ black box' and go beyond restricted quantitative assessment of $C Q$ - a need that epitomizes the frontier of $C Q$ research. Again, the view of $C Q$, or specifically $X C Q$, as elucidated in this study provides a promising key that could unlock the mysterious CQ black box. Together these two research themes offers ample opportunities for future research programs that will advance our understanding of cross-cultural affairs and the strategic CQ and XCQ we need to manage them effectively (Chapter 8).

\footnotetext{
T
} HIS STUDY HAS BROUGHT TO LIGHT A NUMBER OF INSIGHTS INTO CROSS-CULTURAL INTERACTION AND the pertinent issues related to it - insights that have either been under-explored or escaped prior research attention altogether. These insights suggest fresh directions not only in current practice but also future research on the nature and implications of culture and learning in organizations. 



\section{AUTHOR'S BIOGRAPHY}

\section{B}

ORN IN BANGKOK, THAILAND, NANTAWAN NOI KWANJAI -

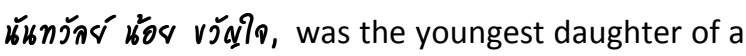

taxi driver and his wife, who served all her life as a civil servant to the Thai Royal Family. Noi was a studious student until her high school years when her curiosity about the meaning of life moved her to search for it in the Thai political movement of the 1970's. She ended up spending a few months as a captive political prisoner, followed by a brief attempt to fit in with the Thai communist insurgency in the Southern jungle of Thailand. Having failed miserably as a freedom fighter, she ventured into many foreign lands and cultures, living lives that spanned the experiences of an illegal immigrant labourer in London, a graduate student in Hawai'i, a consultant in Singapore, a general manager in Thailand and a researcher and teacher in the Netherlands.

Noi is now a naturalized Dutch citizen. Prior to joining Maastricht University, she obtained academic qualifications in diverse disciplines, including English Literature (at Thammasat University, Thailand), Business Administration, Management Information Systems, and Communication \& Information Science (all at the University of Hawai'i at Mānoa, USA). Her earlier work experience was primarily in management and industry research at locations in the USA, Singapore, Thailand, and the Netherlands. In recent years, she has carried out market research projects on the Dutch industries and businesses for an international market research consultancy, as well as designed, coordinated and conducted problem-based learning courses at Maastricht University, where she also worked on her doctoral research project. After completion of her doctoral study, she plans to henceforth develop a career combining research, teaching, and consultancy. Her main interests will evolve around the broad field of culture and learning in organizations, with specific focus on the theme of cultural intelligence (CQ) in general and cross-cultural intelligence (XCQ) in particular.

A t the moment, Nol lives a PeAceful life With her Dutch partner, ARnold Vermeer, and their four cats - Pepper, Prince, Romeo and Julia - in Europe.

January 2011

Maastricht, the Netherlands 